\title{
TABLE OF CONTENTS
}

\begin{tabular}{|c|c|c|}
\hline \multirow[t]{8}{*}{$\begin{array}{l}\text { Target } \\
\text { Chk1 Kinease } \\
\end{array}$} & Docking Program & $\begin{array}{r}\text { Page Numbers } \\
2-11\end{array}$ \\
\hline & $\begin{array}{l}\text { Dock4 } \\
\text { Docklt }\end{array}$ & $\begin{array}{r}2 \\
3\end{array}$ \\
\hline & $\begin{array}{l}\text { Docklt } \\
\text { FlexX }\end{array}$ & $\begin{array}{l}3 \\
4\end{array}$ \\
\hline & & 5 \\
\hline & & 6 \\
\hline & $\begin{array}{l}\text { Glide } \\
\text { Gold }\end{array}$ & $\begin{array}{l}7 \\
8\end{array}$ \\
\hline & & 9 \\
\hline & $\begin{array}{l}\text { MoeDock } \\
\text { MVP }\end{array}$ & $\begin{array}{l}10 \\
11\end{array}$ \\
\hline \multirow[t]{8}{*}{ Factor Xa } & & 12-21 \\
\hline & Dock4 & 12 \\
\hline & $\begin{array}{l}\text { DockIt } \\
\text { Flex }\end{array}$ & $\begin{array}{l}13 \\
14\end{array}$ \\
\hline & $\begin{array}{l}\text { FlexX } \\
\text { Flo+ }\end{array}$ & $\begin{array}{l}14 \\
15\end{array}$ \\
\hline & Fred & 16 \\
\hline & $\begin{array}{l}\text { Glide } \\
\text { Gold }\end{array}$ & $\begin{array}{l}17 \\
18\end{array}$ \\
\hline & $\begin{array}{l}\text { Gou } \\
\text { LigFit } \\
\text { MoeDock }\end{array}$ & $\begin{array}{l}19 \\
20\end{array}$ \\
\hline & $\begin{array}{l}\text { Moedock } \\
\text { MVP }\end{array}$ & 21 \\
\hline \multirow[t]{8}{*}{ Gyrase B } & & 22-31 \\
\hline & Dock4 & 22 \\
\hline & $\begin{array}{l}\text { DockIt } \\
\text { FlexX }\end{array}$ & $\begin{array}{l}23 \\
24\end{array}$ \\
\hline & Flo+ & 25 \\
\hline & $\begin{array}{l}\text { Fred } \\
\text { Glide }\end{array}$ & $\begin{array}{l}26 \\
27\end{array}$ \\
\hline & & 28 \\
\hline & LigFit & $\begin{array}{l}29 \\
30\end{array}$ \\
\hline & $\begin{array}{l}\text { MoeDock } \\
\text { MVP }\end{array}$ & $\begin{array}{l}30 \\
31\end{array}$ \\
\hline \multirow{8}{*}{ HCV Polymerase } & & $32-41$ \\
\hline & $\begin{array}{l}\text { Dock4 } \\
\text { Docktt }\end{array}$ & $\begin{array}{l}32 \\
33\end{array}$ \\
\hline & $\begin{array}{l}\text { Docklt } \\
\text { FlexX }\end{array}$ & $\begin{array}{l}33 \\
34\end{array}$ \\
\hline & $\mathrm{Flo}^{+}$ & $\begin{array}{l}35 \\
36\end{array}$ \\
\hline & $\begin{array}{l}\text { Fred } \\
\text { Grile }\end{array}$ & $\begin{array}{l}36 \\
37\end{array}$ \\
\hline & & 38 \\
\hline & $\begin{array}{l}\text { LigFit } \\
\text { MoeDock }\end{array}$ & $\begin{array}{l}39 \\
40\end{array}$ \\
\hline & $\begin{array}{l}\text { Moedock } \\
\text { MVP }\end{array}$ & $\begin{array}{l}40 \\
41\end{array}$ \\
\hline \multirow[t]{7}{*}{ Methionyl tRNA synthetase } & & $42-51$ \\
\hline & $\begin{array}{l}\text { Dock4 } \\
\text { DockIt }\end{array}$ & $\begin{array}{l}42 \\
43\end{array}$ \\
\hline & FlexX & $\begin{array}{l}43 \\
44\end{array}$ \\
\hline & $\begin{array}{l}\text { Flo+ } \\
\text { Fred }\end{array}$ & $\begin{array}{l}45 \\
46\end{array}$ \\
\hline & & \\
\hline & & $\begin{array}{l}48 \\
49\end{array}$ \\
\hline & $\begin{array}{l}\text { LigFit } \\
\text { MoeDock }\end{array}$ & $\begin{array}{l}49 \\
50 \\
51\end{array}$ \\
\hline \multirow{8}{*}{ E. coli PDF } & & $52-61$ \\
\hline & Dock4 & 52 \\
\hline & $\begin{array}{l}\text { Docklt } \\
\text { FlexX }\end{array}$ & $\begin{array}{l}53 \\
54\end{array}$ \\
\hline & $\begin{array}{l}F_{1} \\
\text { Flo+ }\end{array}$ & 55 \\
\hline & $\begin{array}{l}\text { Fred } \\
\text { Gide }\end{array}$ & $\begin{array}{l}56 \\
57\end{array}$ \\
\hline & $\begin{array}{l}\text { Ginee } \\
\text { Gold }\end{array}$ & 58 \\
\hline & LigFit & $\begin{array}{l}59 \\
60\end{array}$ \\
\hline & $\begin{array}{l}\text { MoebDock } \\
\text { MVP }\end{array}$ & 60 \\
\hline \multirow[t]{8}{*}{ S. pneumococcus PDF } & & $62-71$ \\
\hline & Dock4 & $\begin{array}{l}62 \\
63\end{array}$ \\
\hline & $\begin{array}{l}\text { Docklt } \\
\text { FlexX }\end{array}$ & 64 \\
\hline & & $\begin{array}{l}65 \\
66\end{array}$ \\
\hline & $\begin{array}{l}\text { Fred } \\
\text { Gride }\end{array}$ & $\begin{array}{l}66 \\
67\end{array}$ \\
\hline & $\begin{array}{l}\text { Glide } \\
\text { Gold }\end{array}$ & $\begin{array}{l}68 \\
69\end{array}$ \\
\hline & $\begin{array}{l}\text { LigFit } \\
\text { MoeDock }\end{array}$ & $\begin{array}{l}69 \\
70 \\
71\end{array}$ \\
\hline & MVP & $72-81$ \\
\hline \multirow{6}{*}{$\underline{\text { PPAR } \delta}$} & Dock4 & $\begin{array}{r}72-81 \\
72\end{array}$ \\
\hline & Docklt & \\
\hline & $\begin{array}{l}\text { FlexX } \\
\text { Flo+ }\end{array}$ & $\begin{array}{l}74 \\
75\end{array}$ \\
\hline & $\begin{array}{l}\text { Fred } \\
\text { Glide }\end{array}$ & $\begin{array}{l}76 \\
77\end{array}$ \\
\hline & Gold & 78 \\
\hline & $\begin{array}{l}\text { LigFit } \\
\text { MoeDock }\end{array}$ & $\begin{array}{l}79 \\
80 \\
81\end{array}$ \\
\hline
\end{tabular}




\section{Dock4}

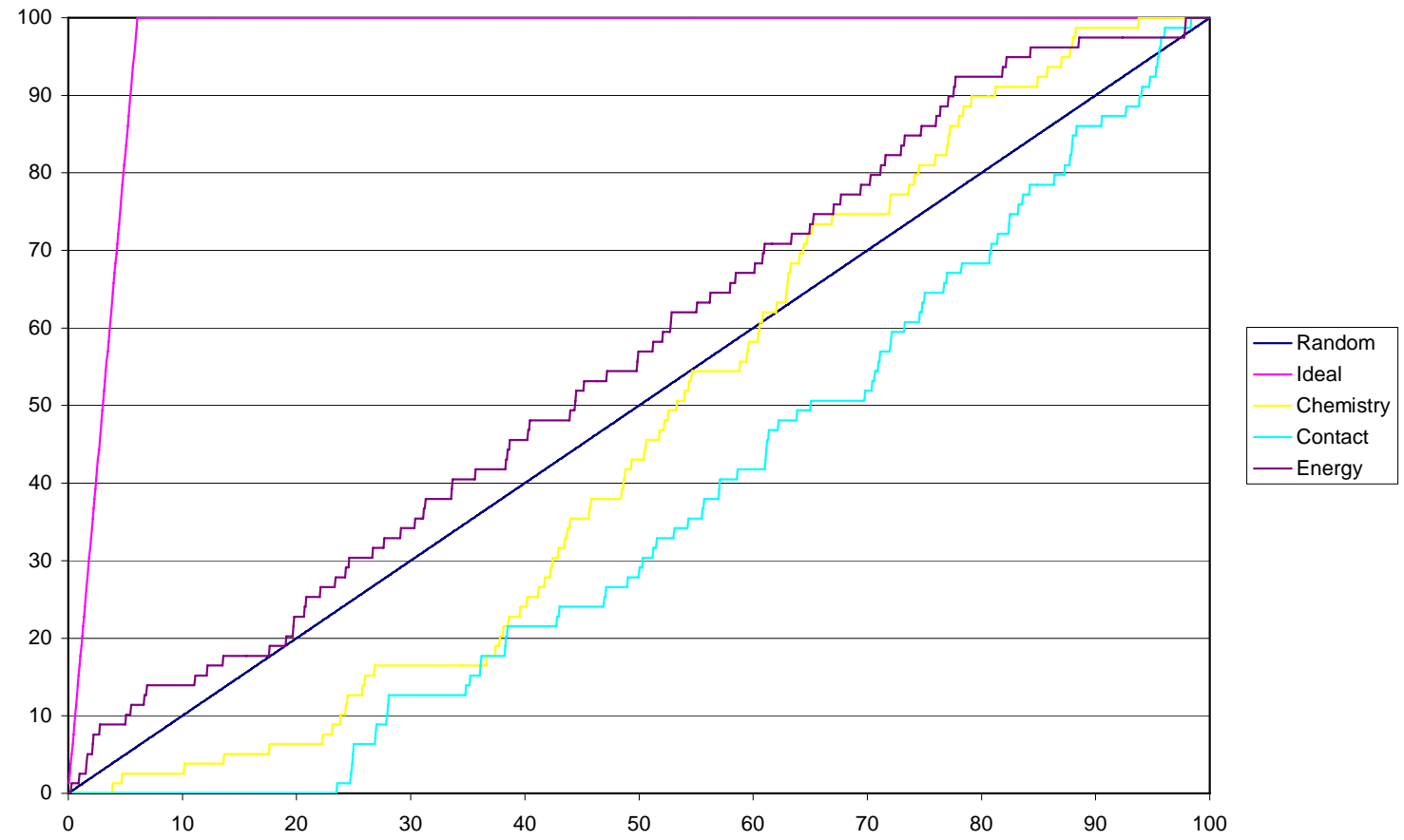

Dock4

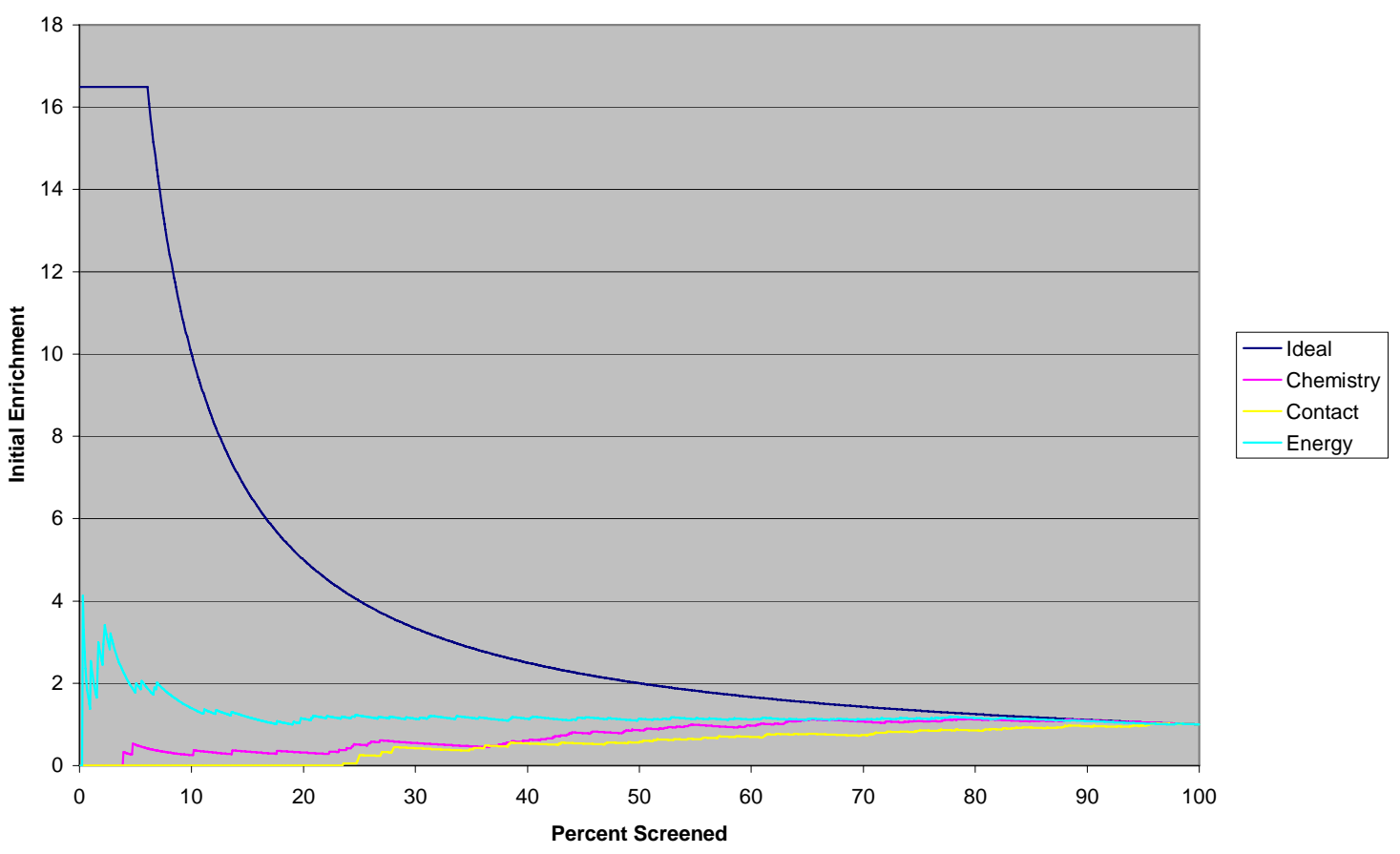


Dockit

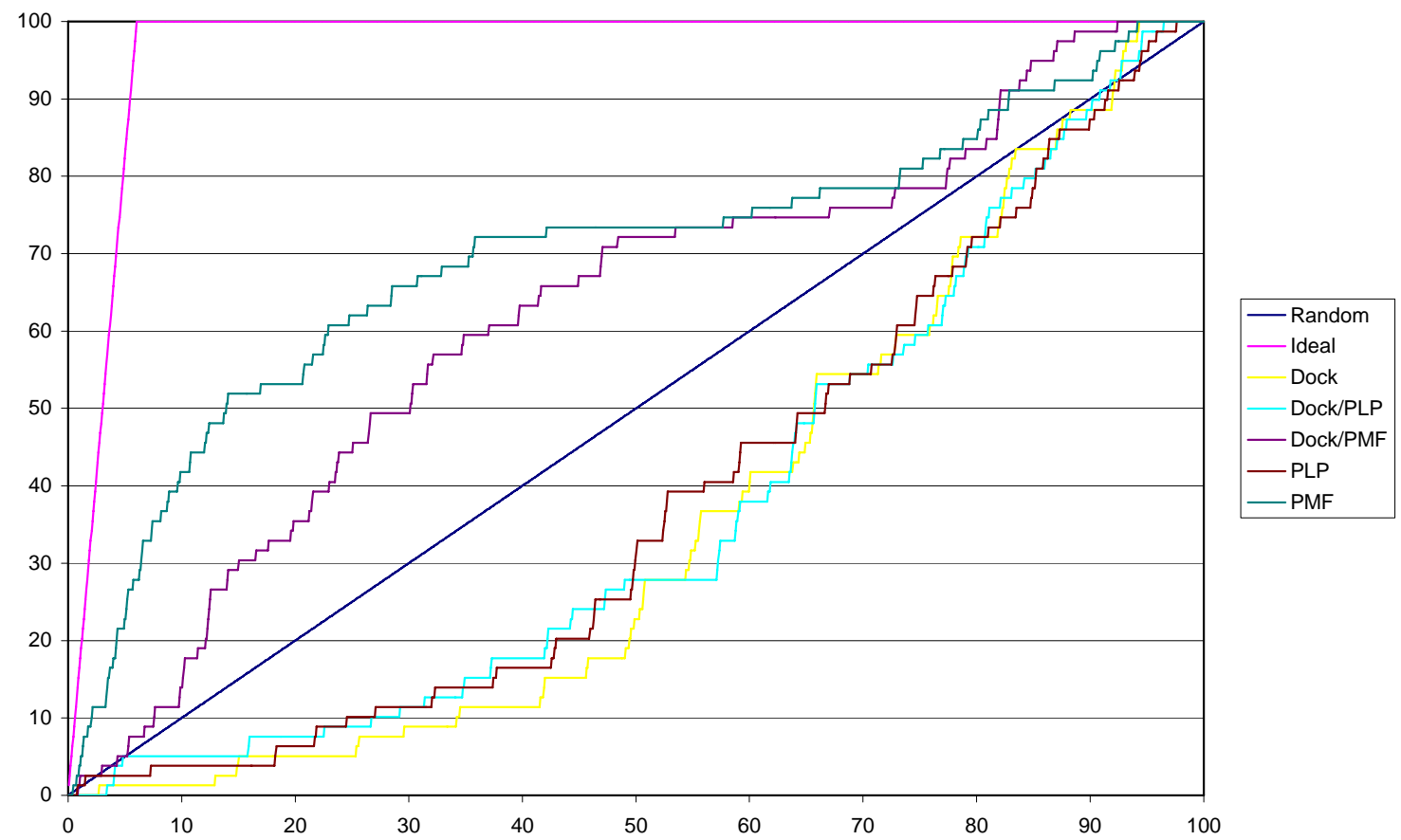

Dockit

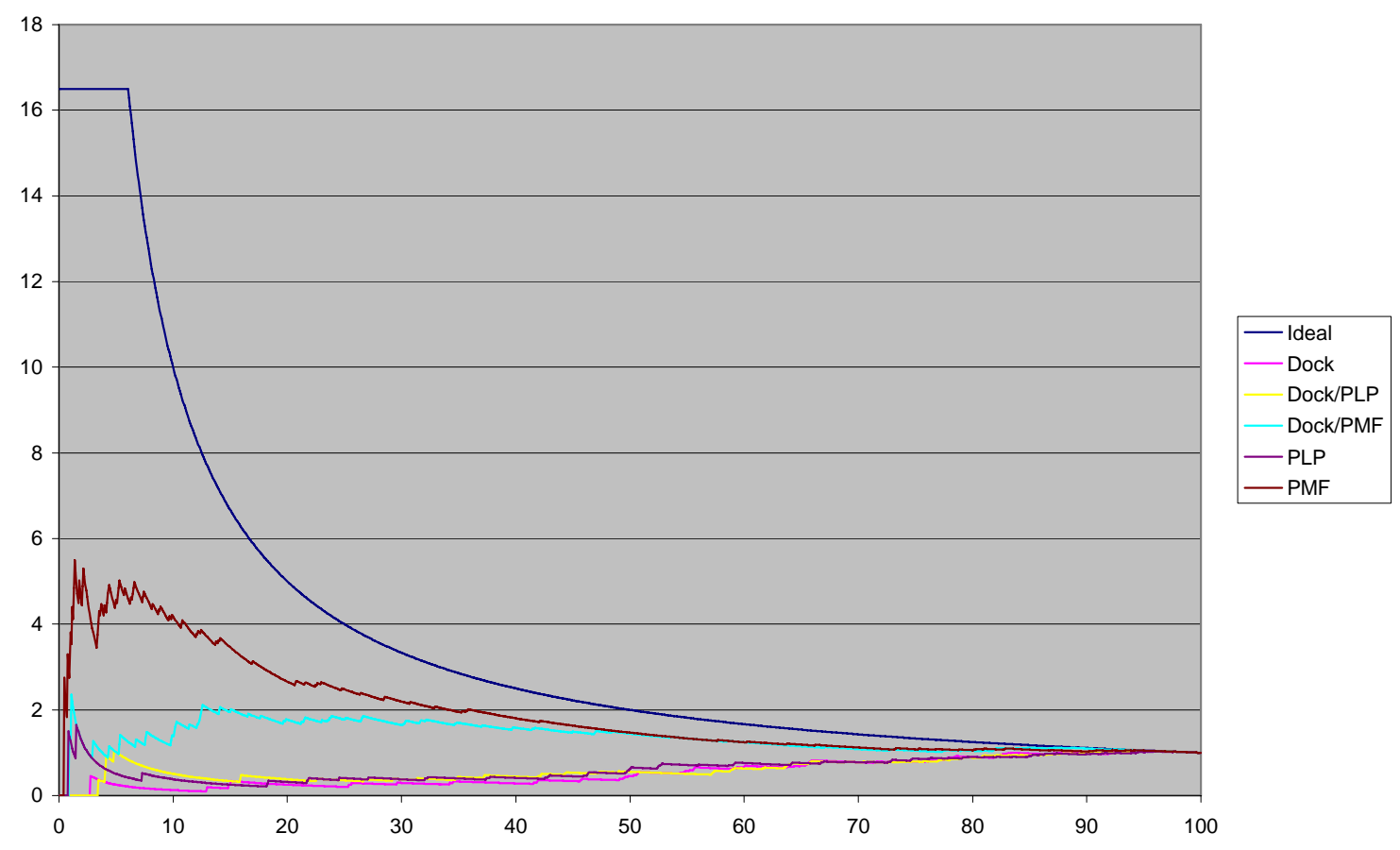


FlexX

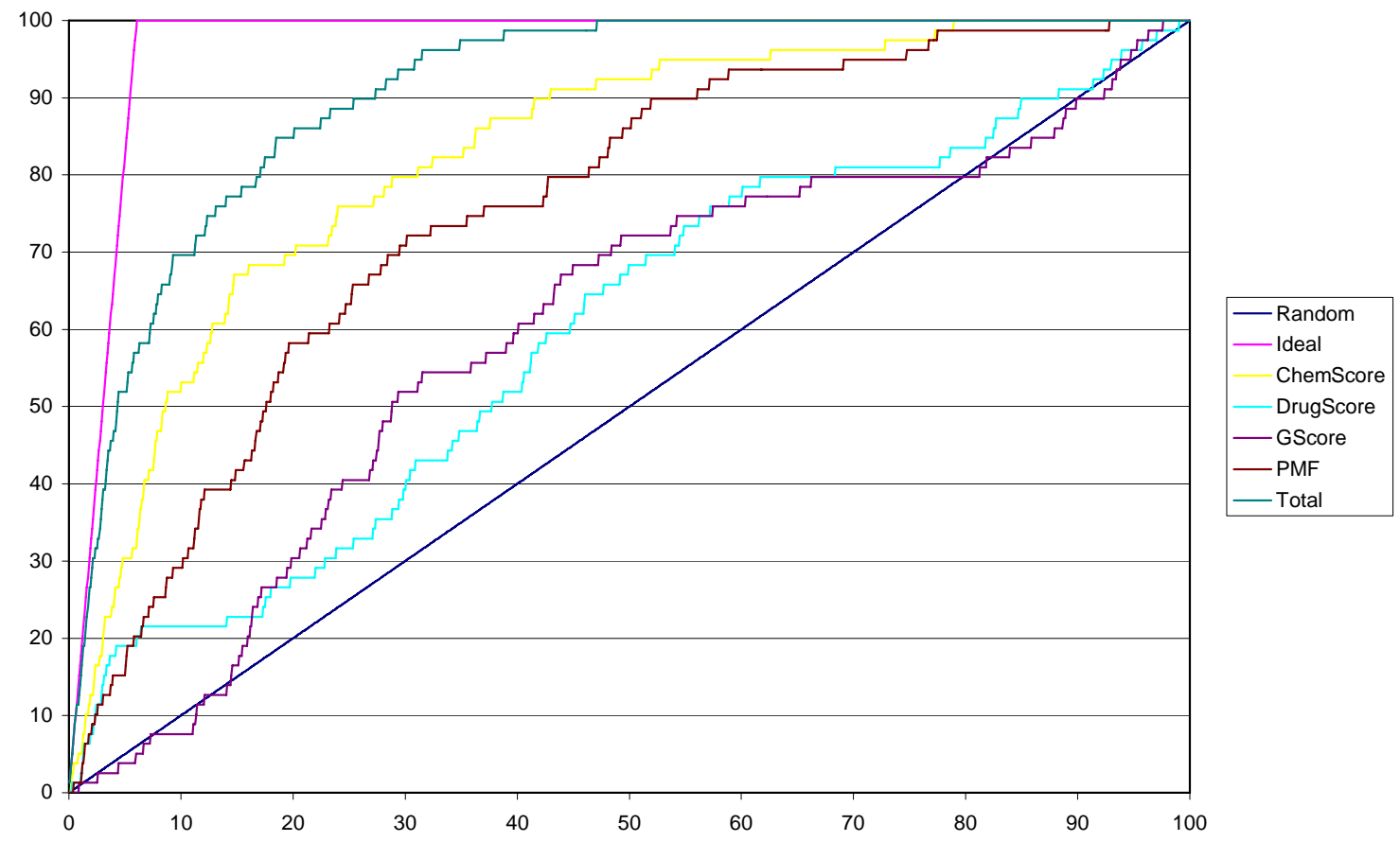

FlexX

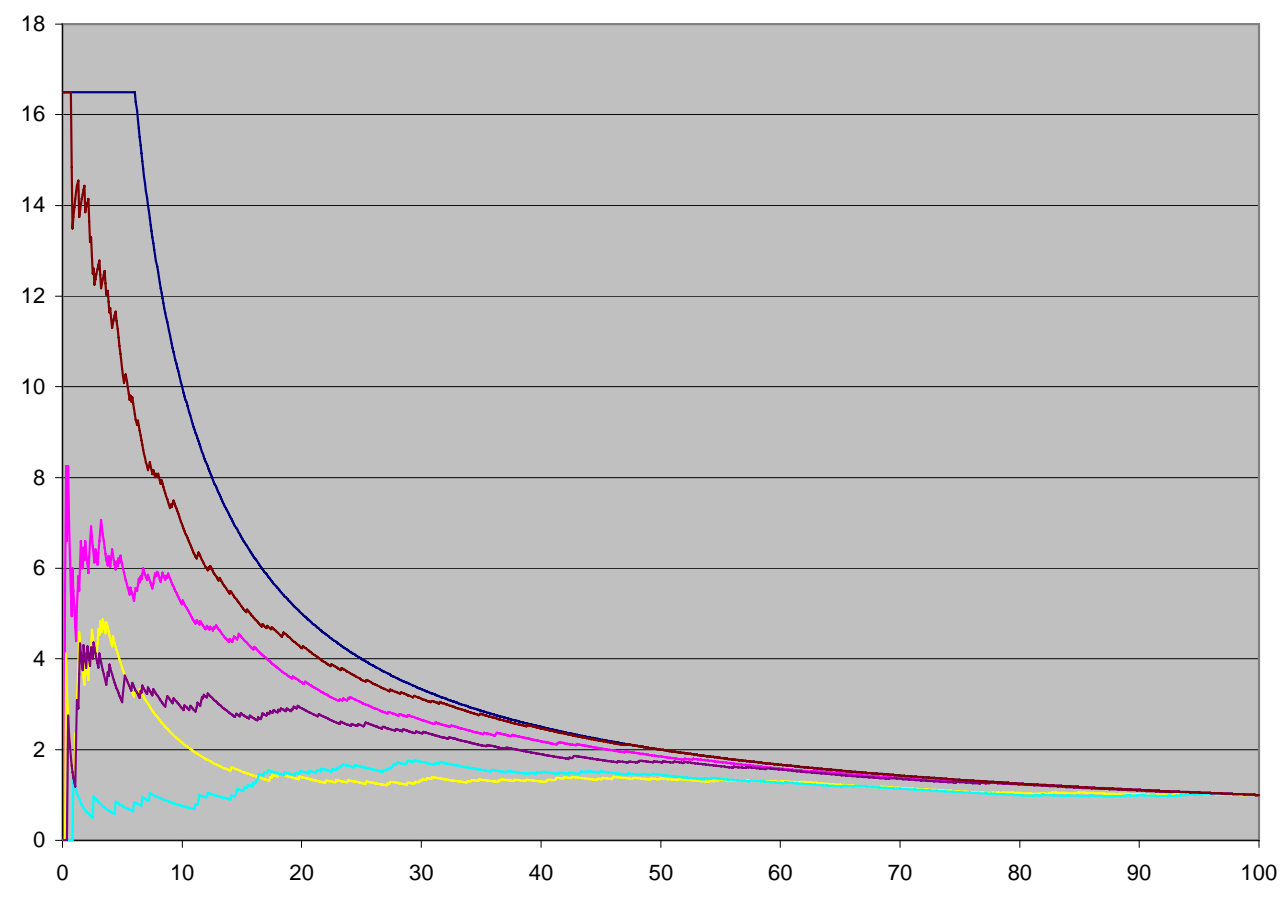

- Ideal
_ ChemScore
DrugScore
Gscore
- PMF
- Total




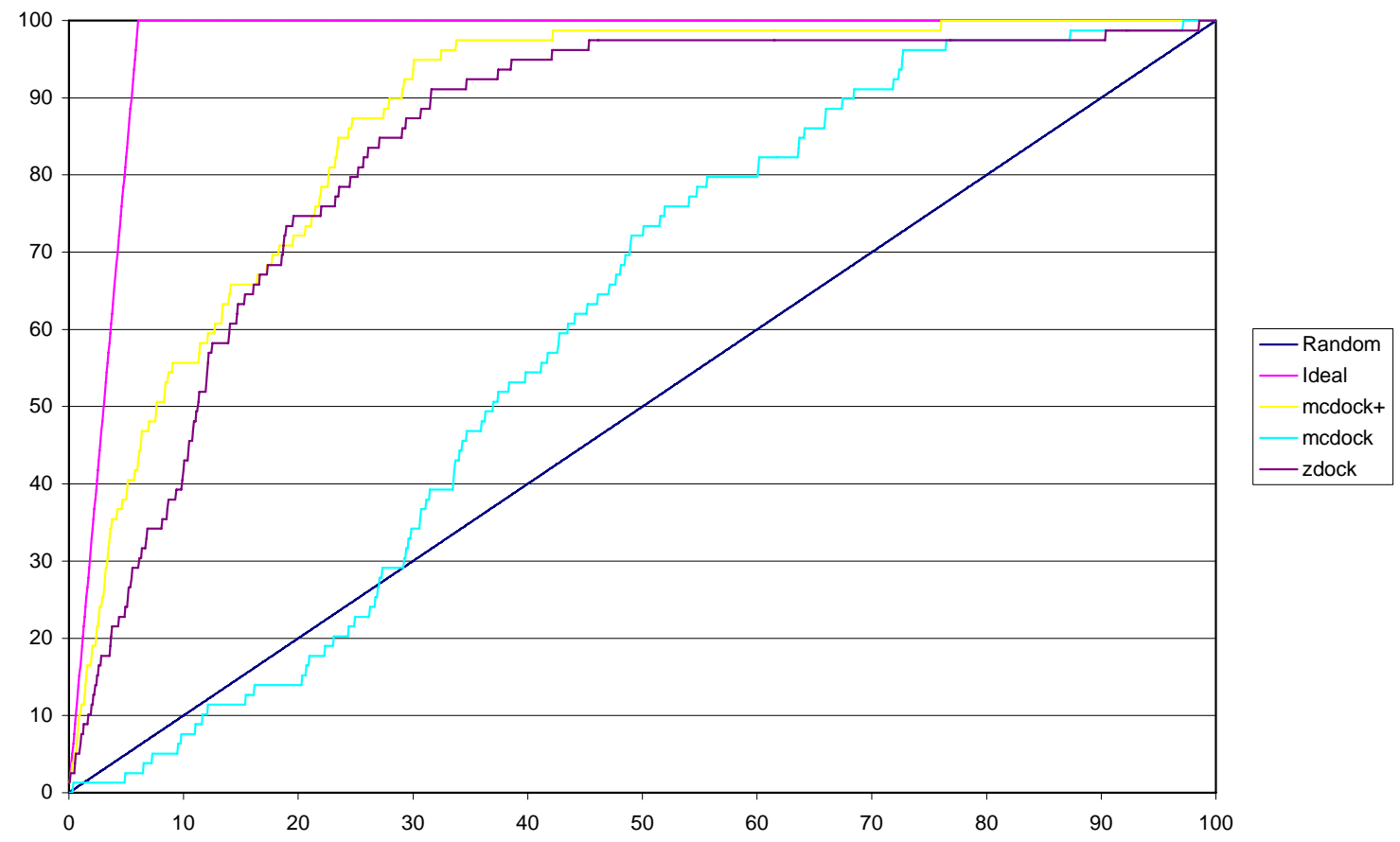

Flo

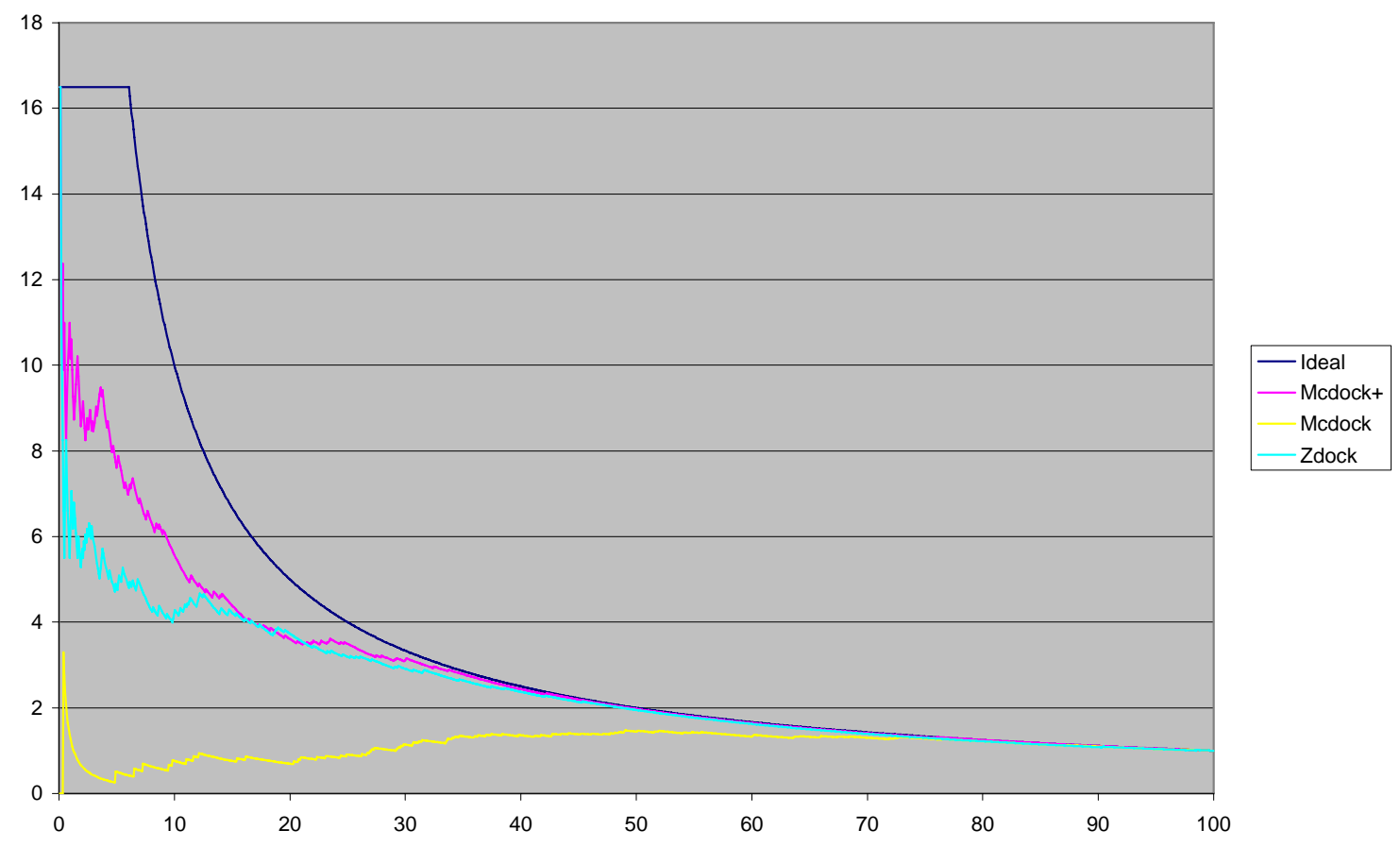


Fred

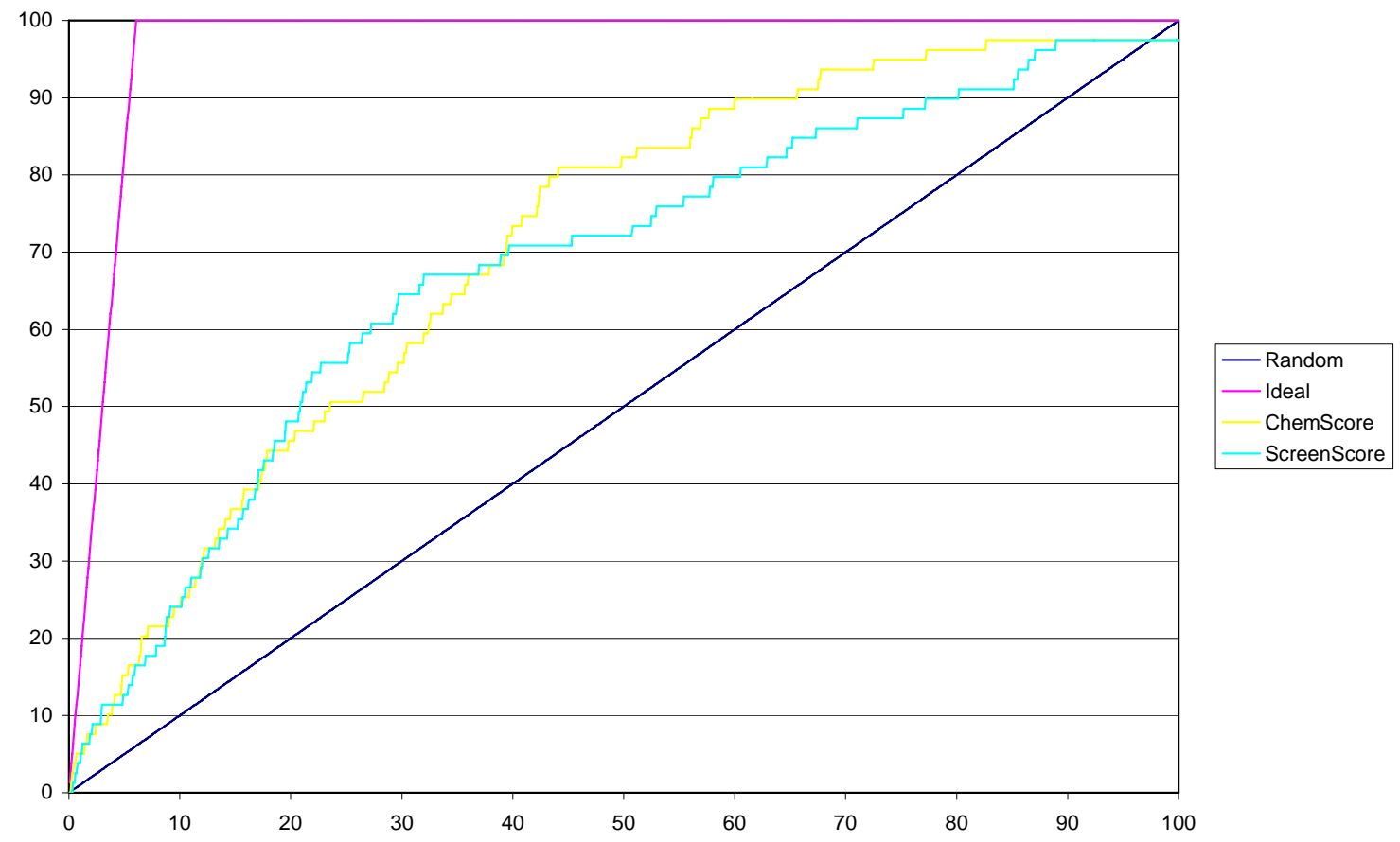

Fred

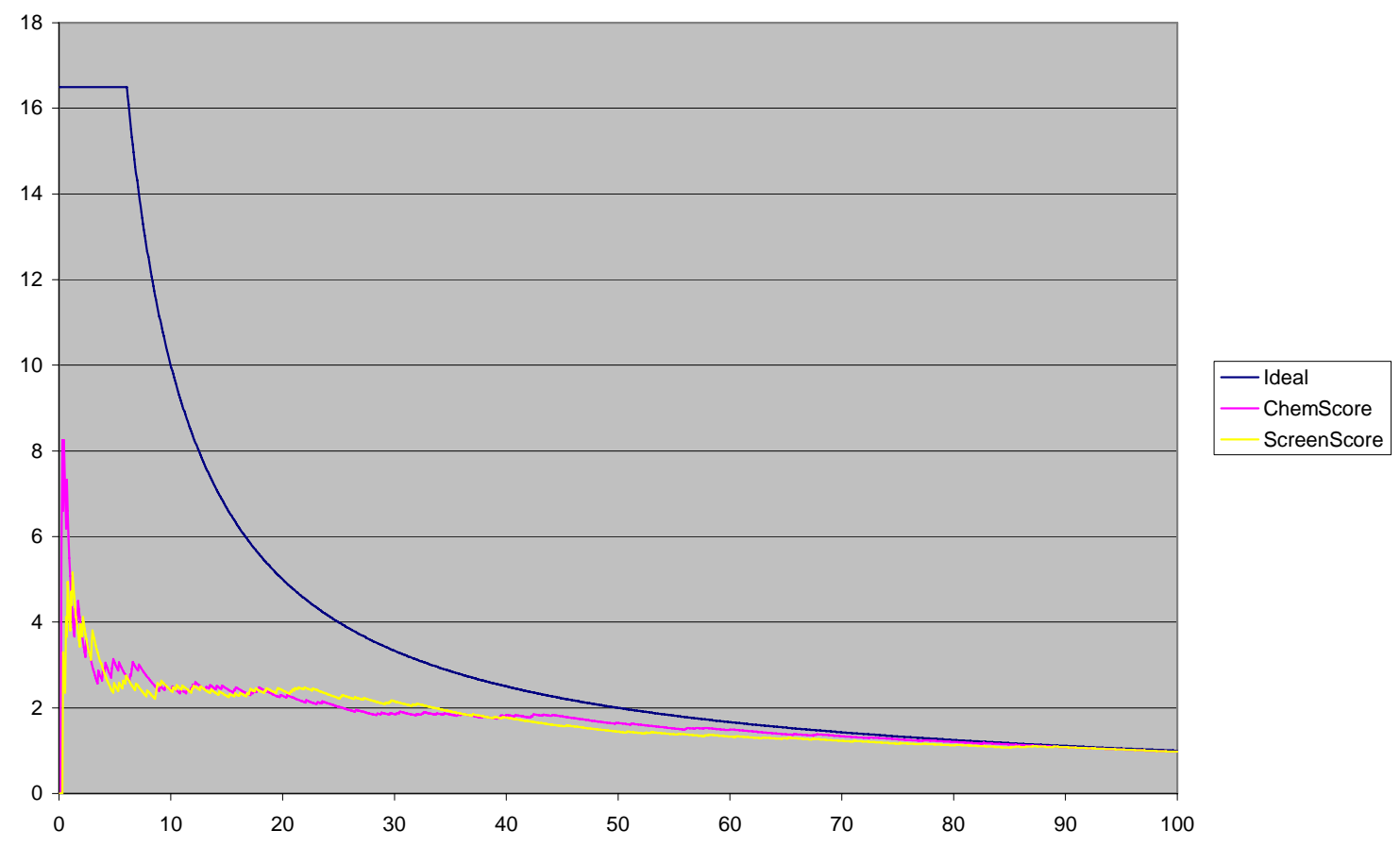




\section{Glide}

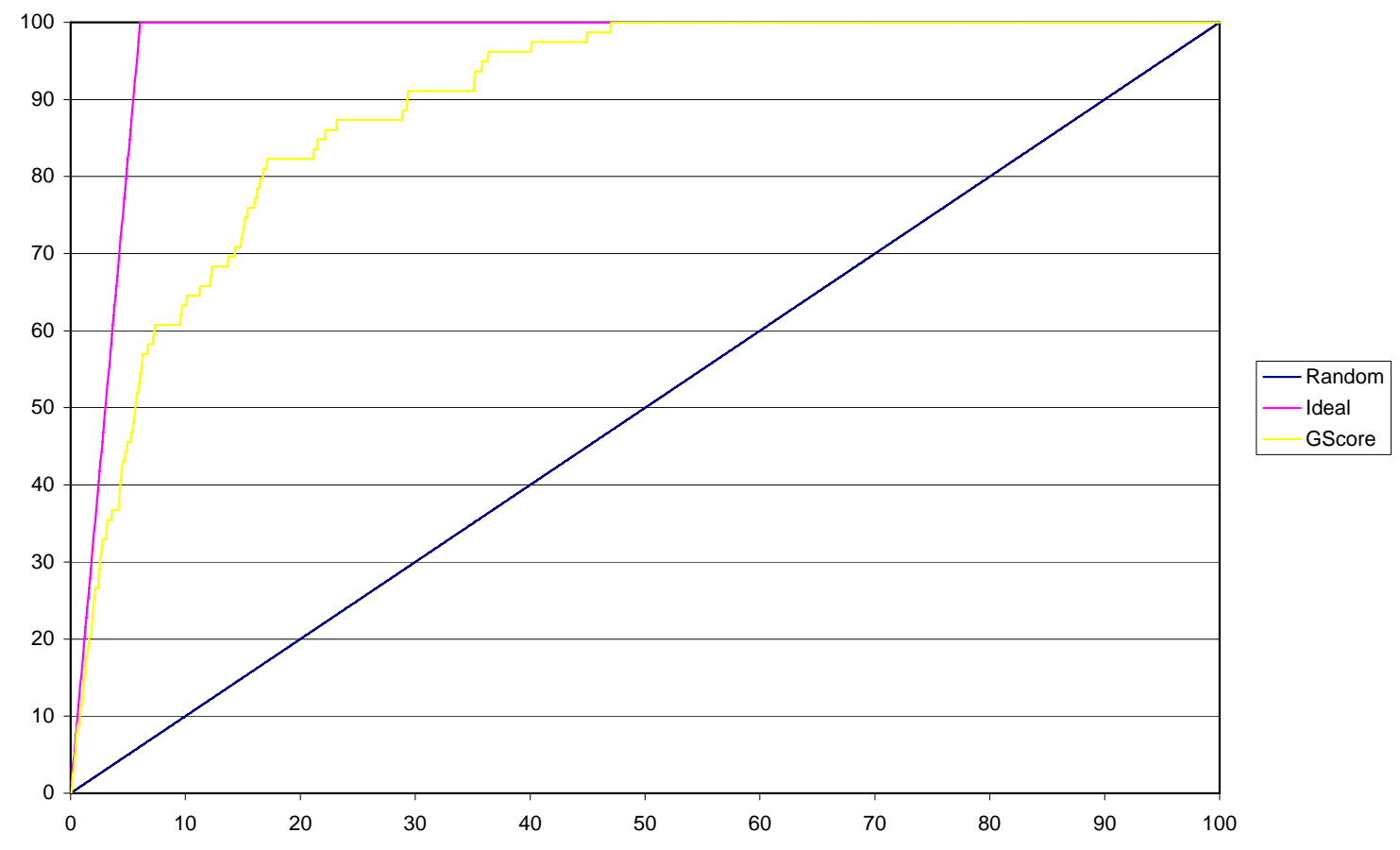

Glide

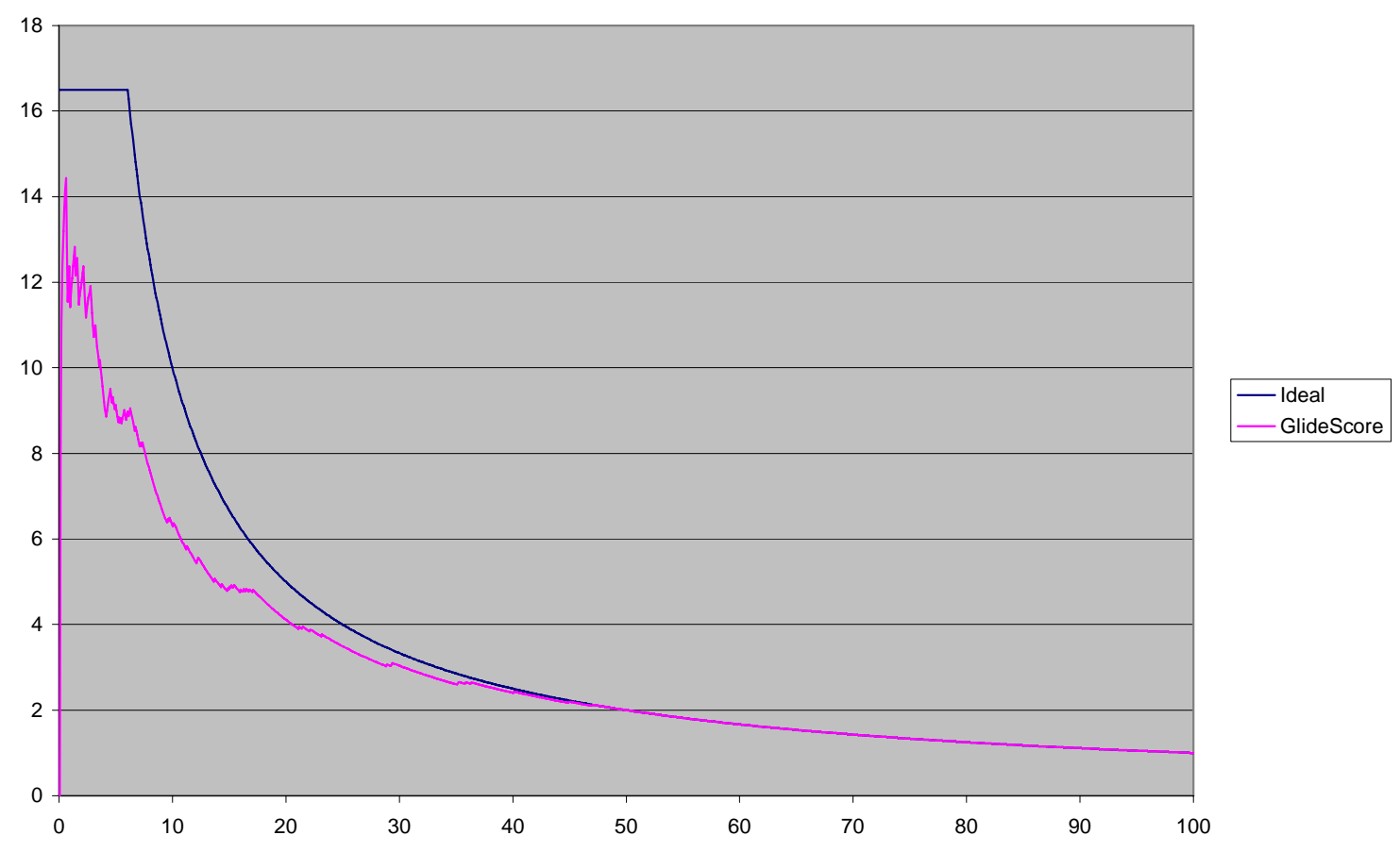


Gold

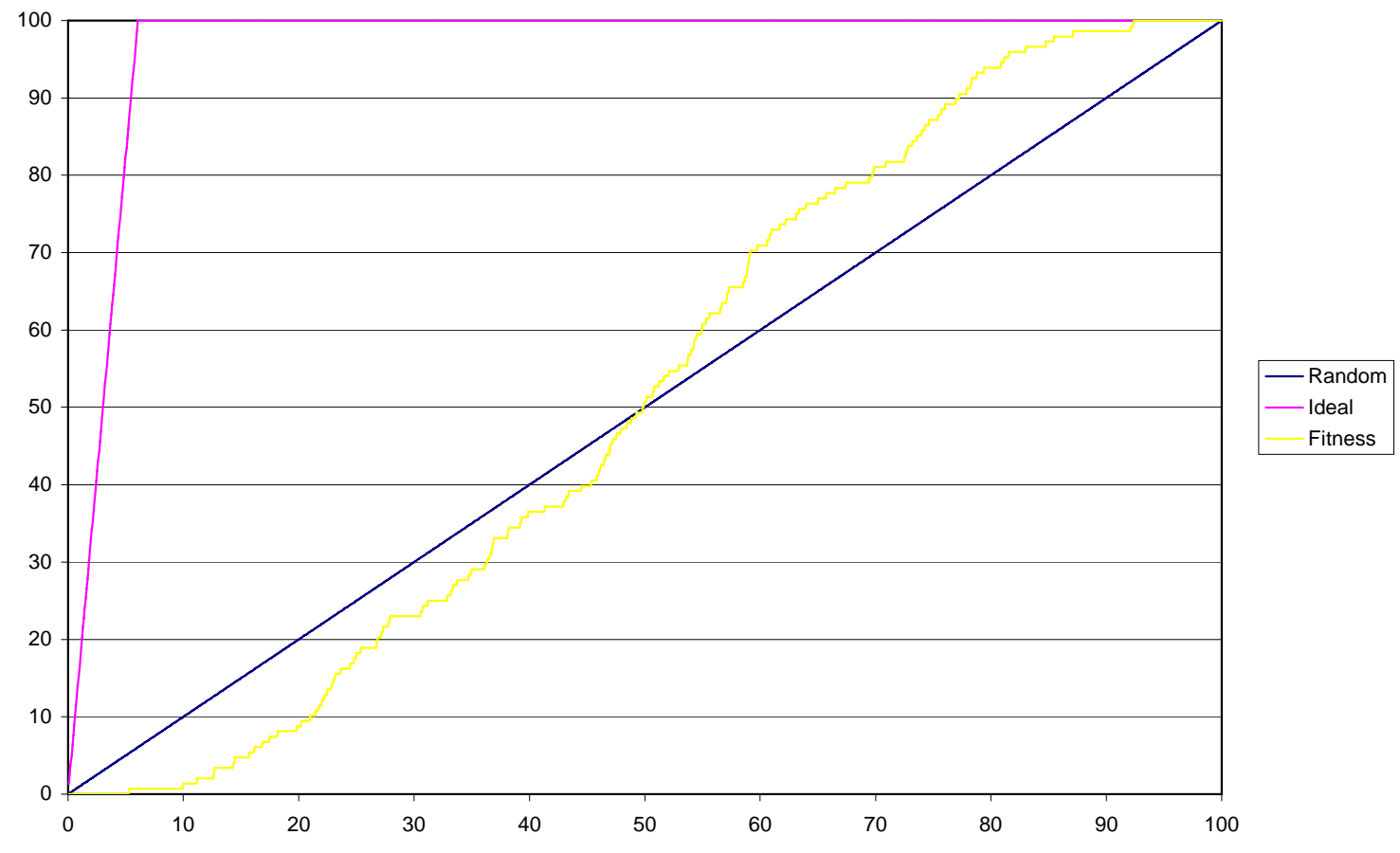

Gold

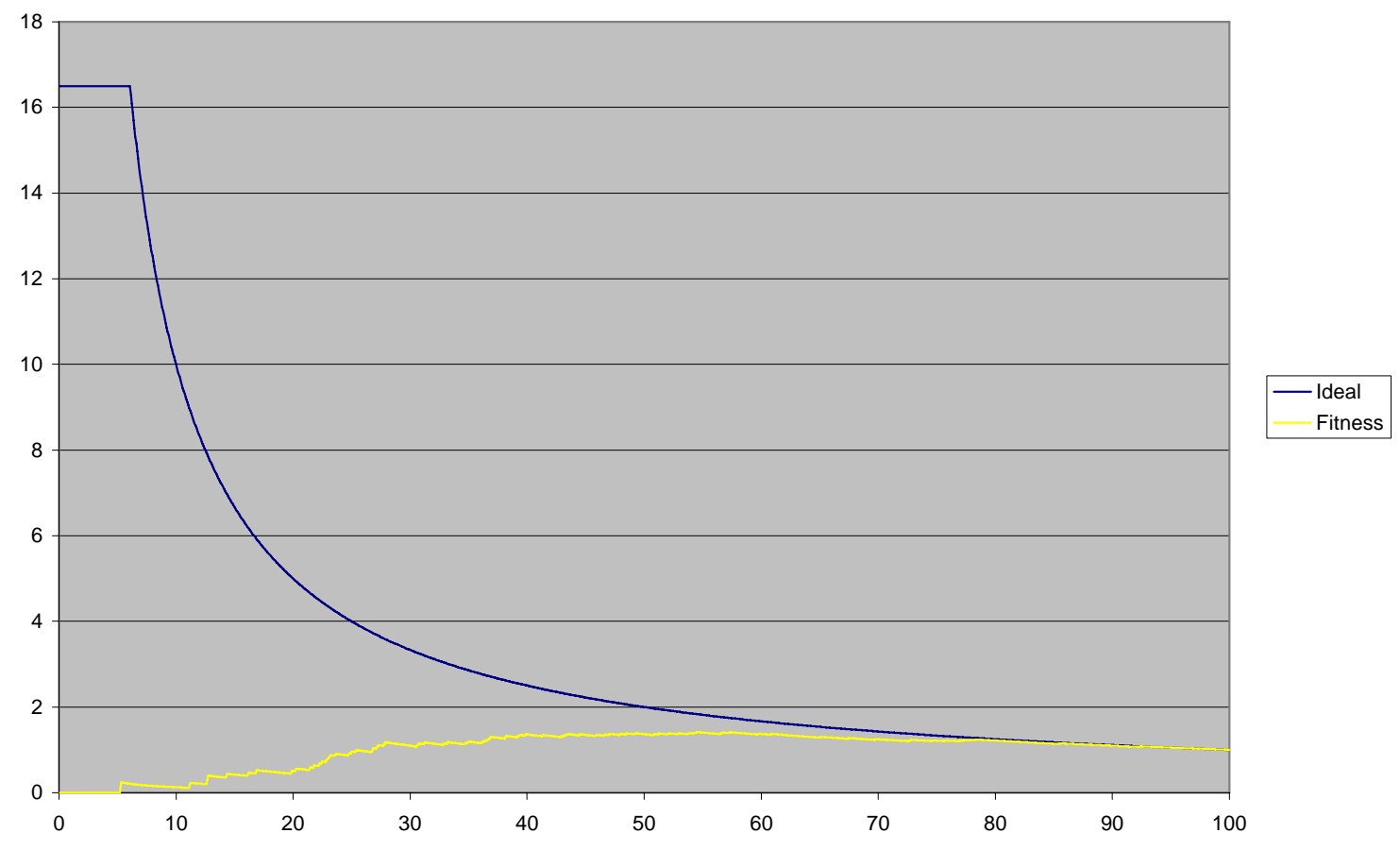




\section{LigFit}

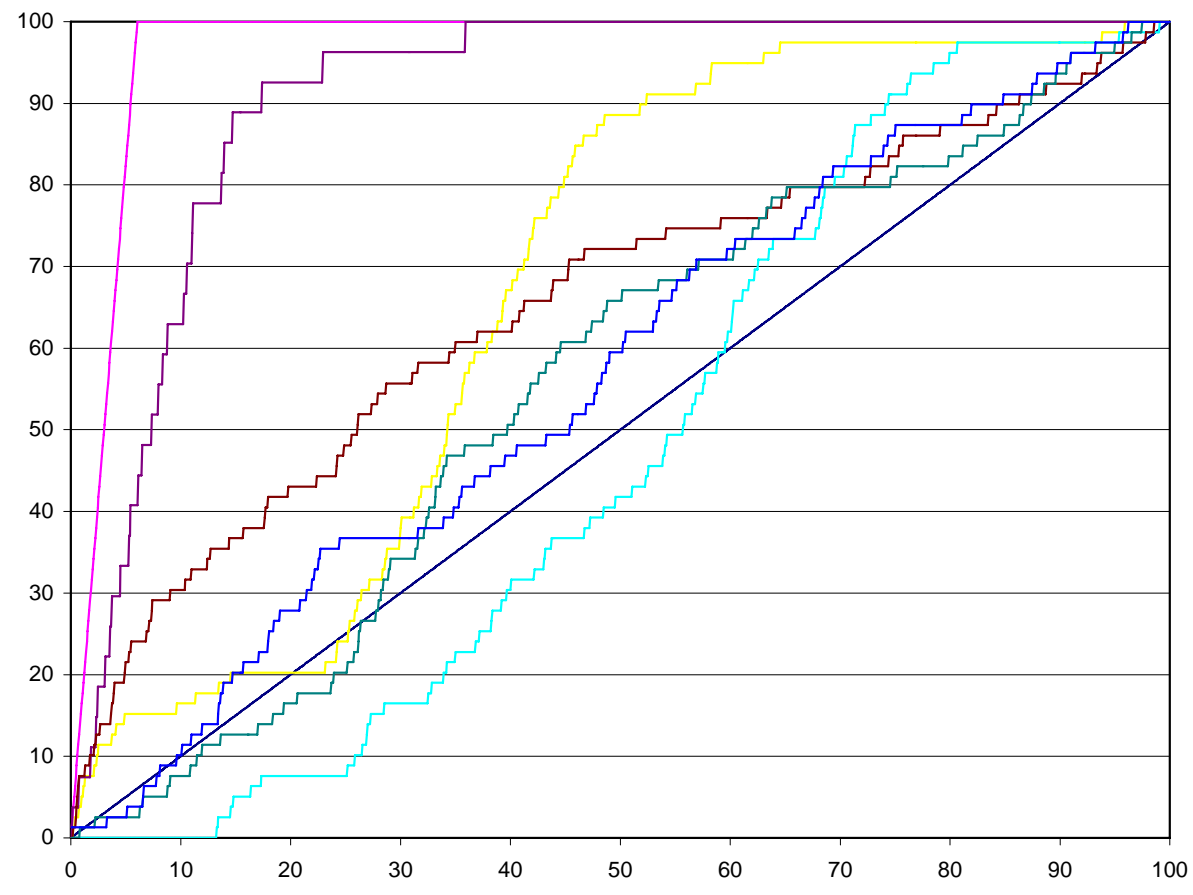

- Random
- Ideal
DrugScore, CFF
DrugScore, Dreiding
- LigScore2, CFF
- LigScore2, Dreiding
PMF, CFF
PMF, Dreiding

Ligfit

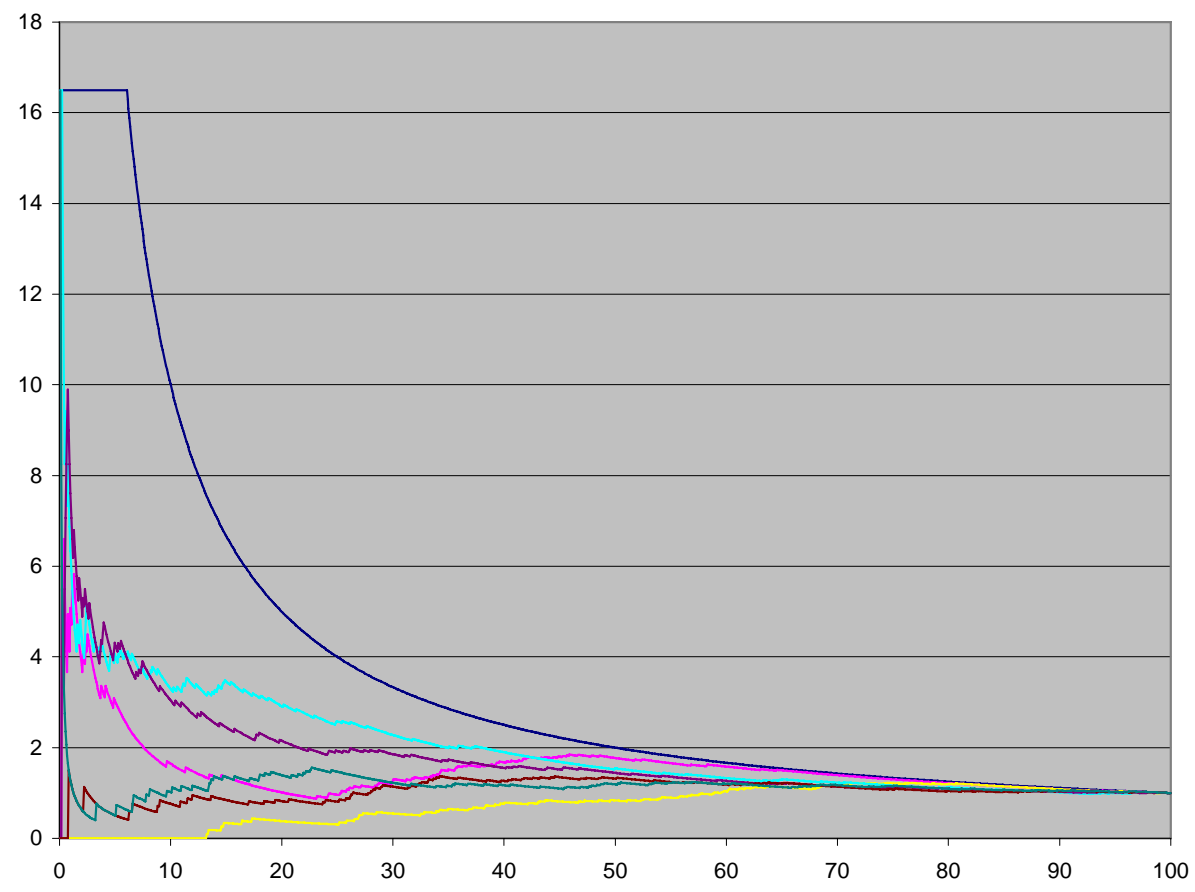

— Ideal
— DrugScore, CFF
DrugScore, Dreiding
— Ligscore2, CFF
- Ligscore2, Dreiding
- PMF, CFF
- PMF, Dreiding


MOE

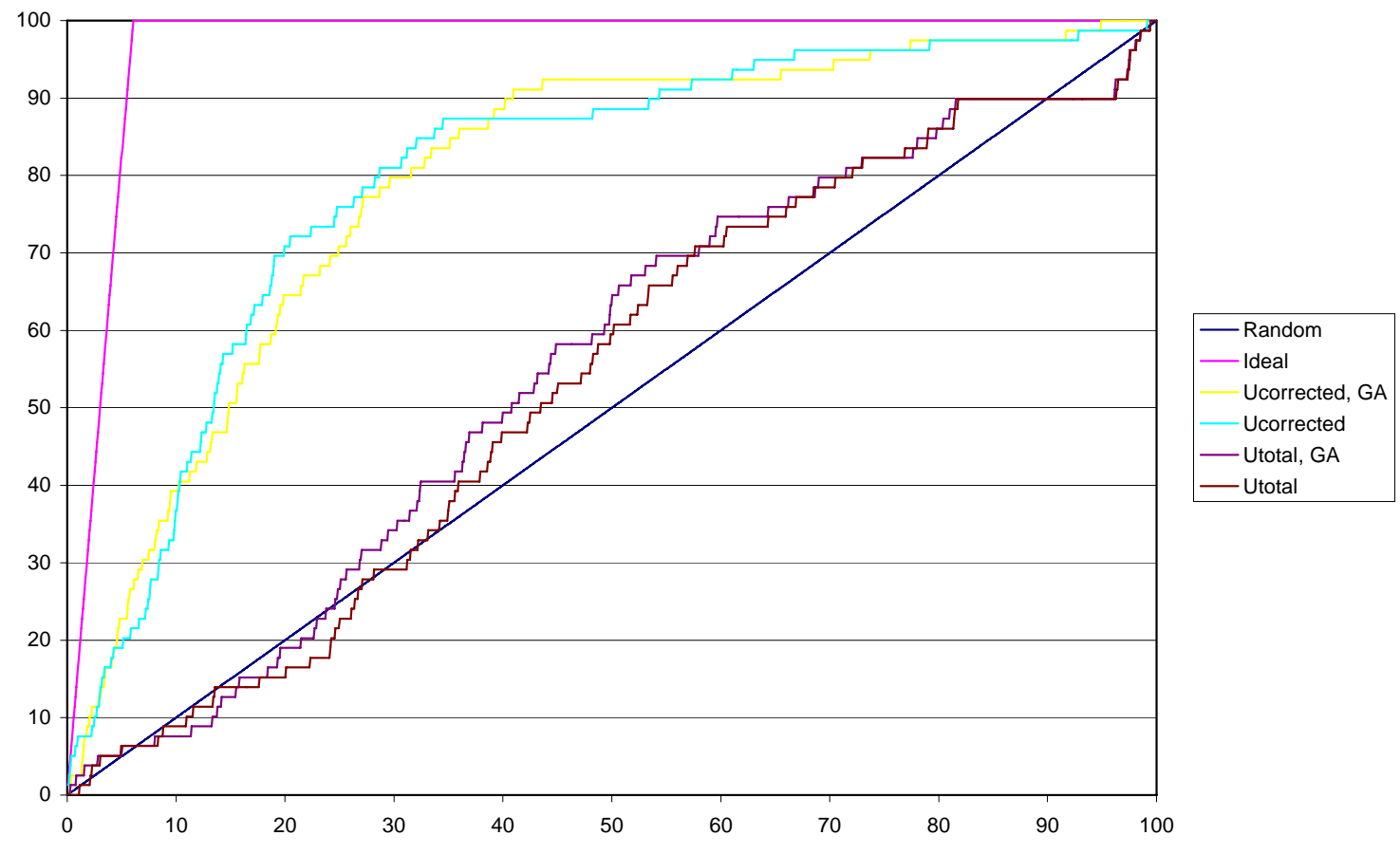

MOE

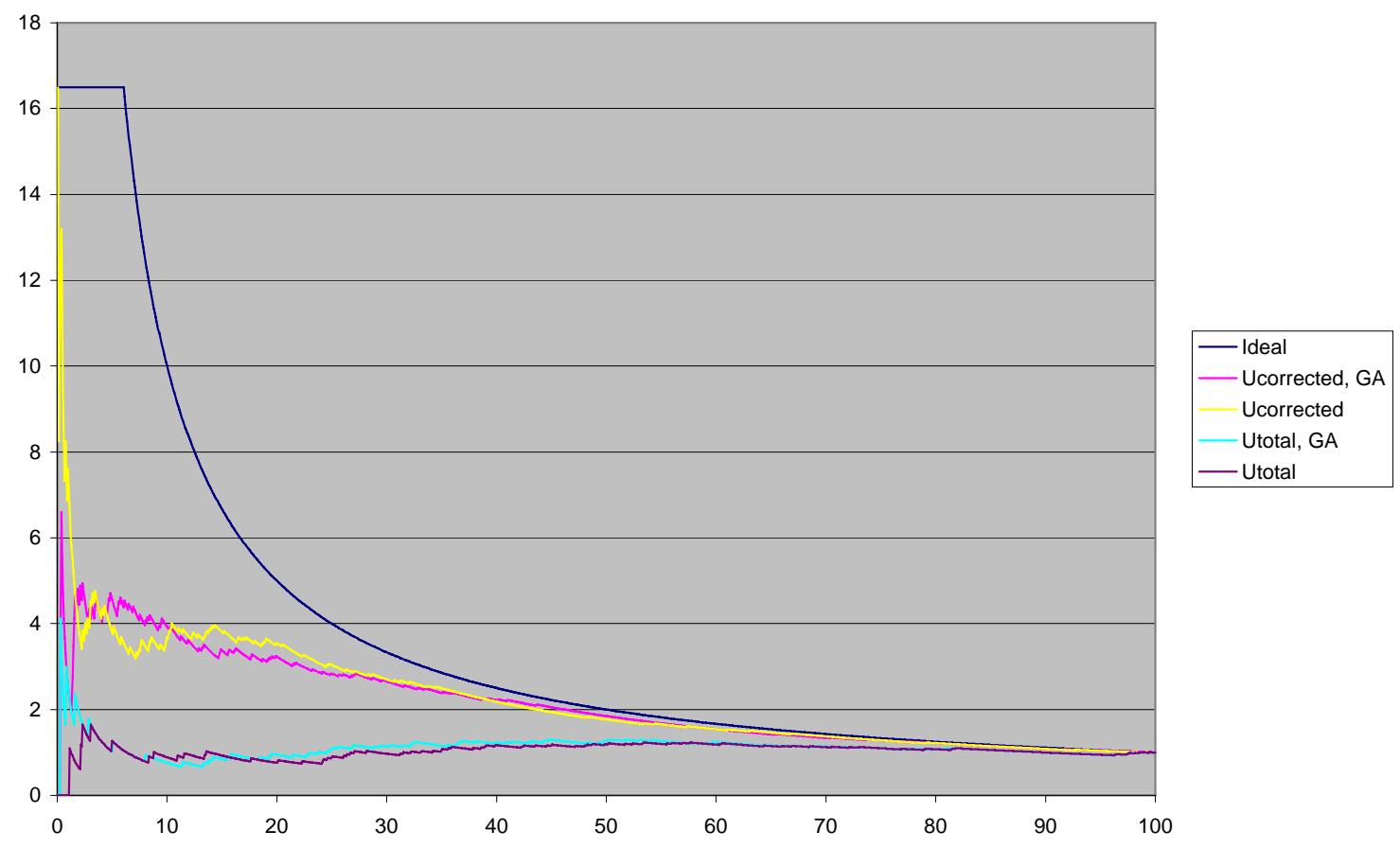


MVP

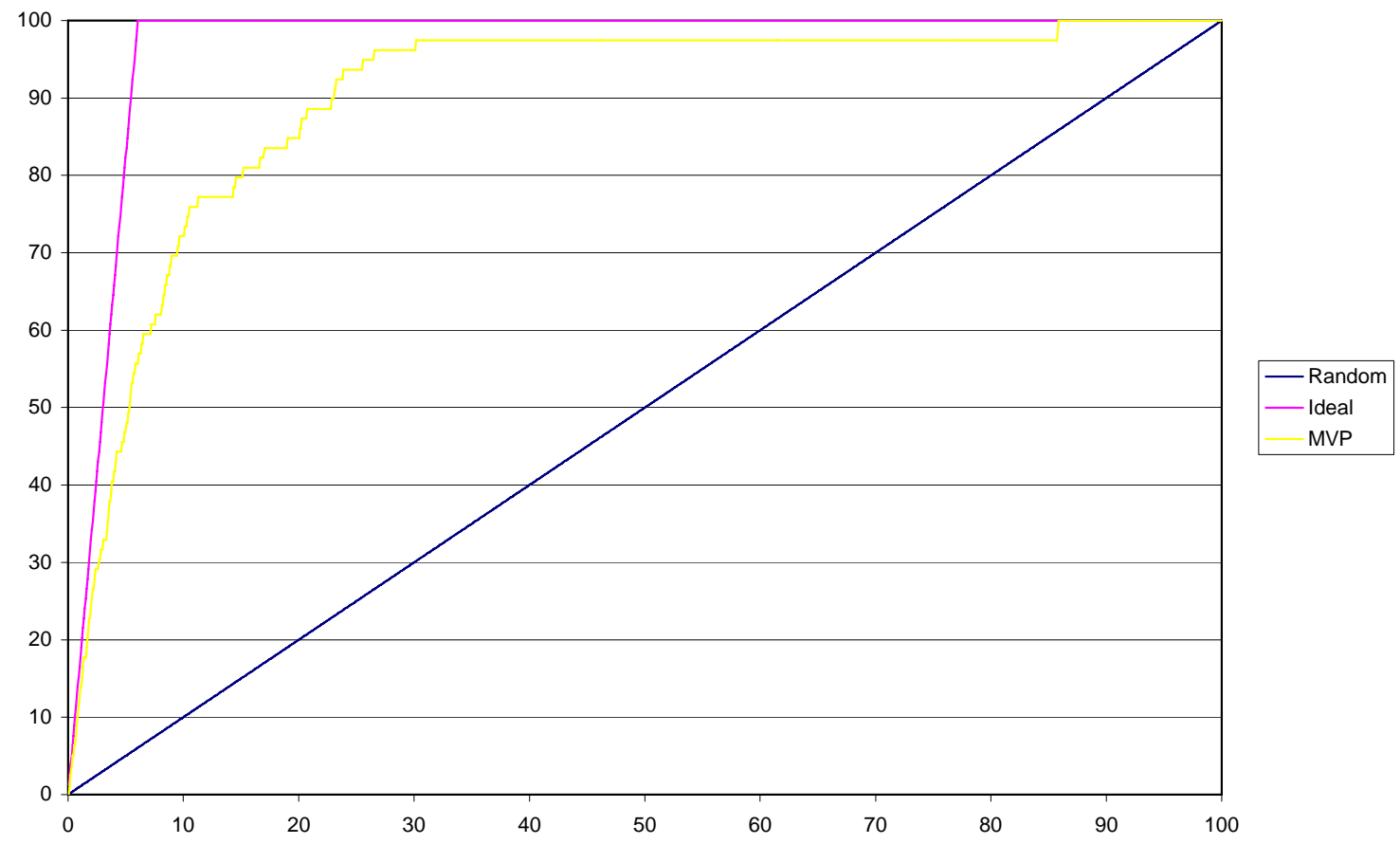

MVP

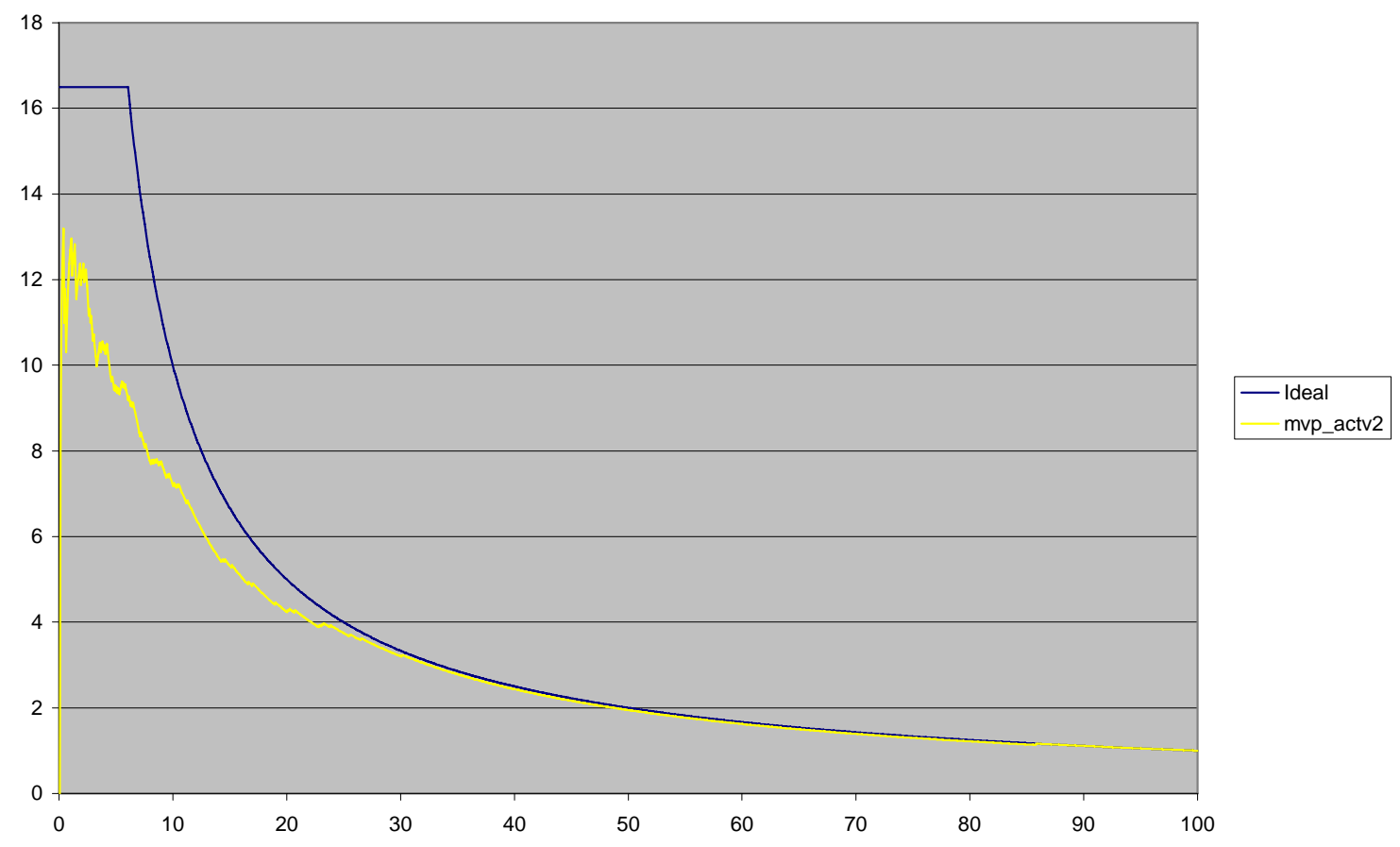


Dock4

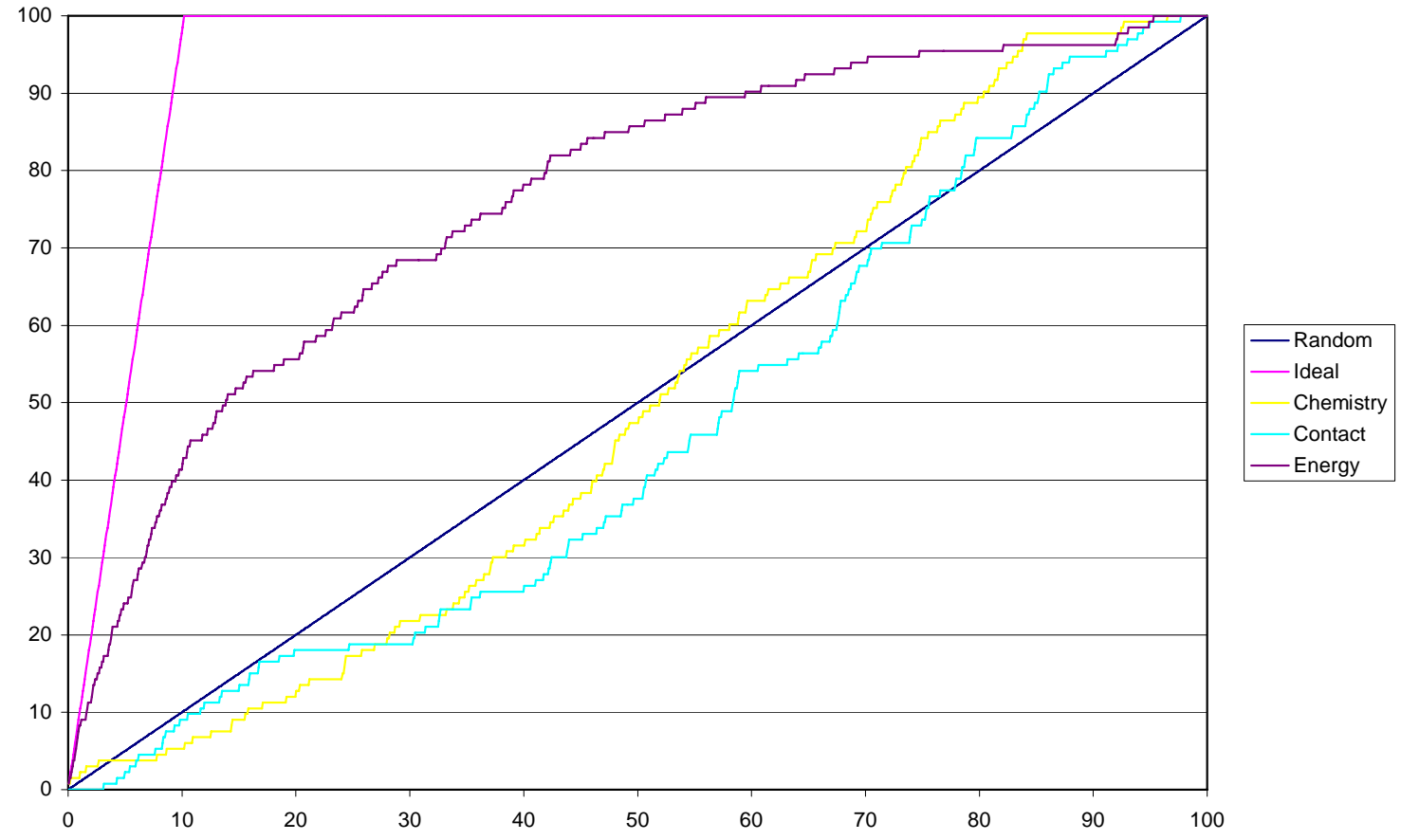

Dock4

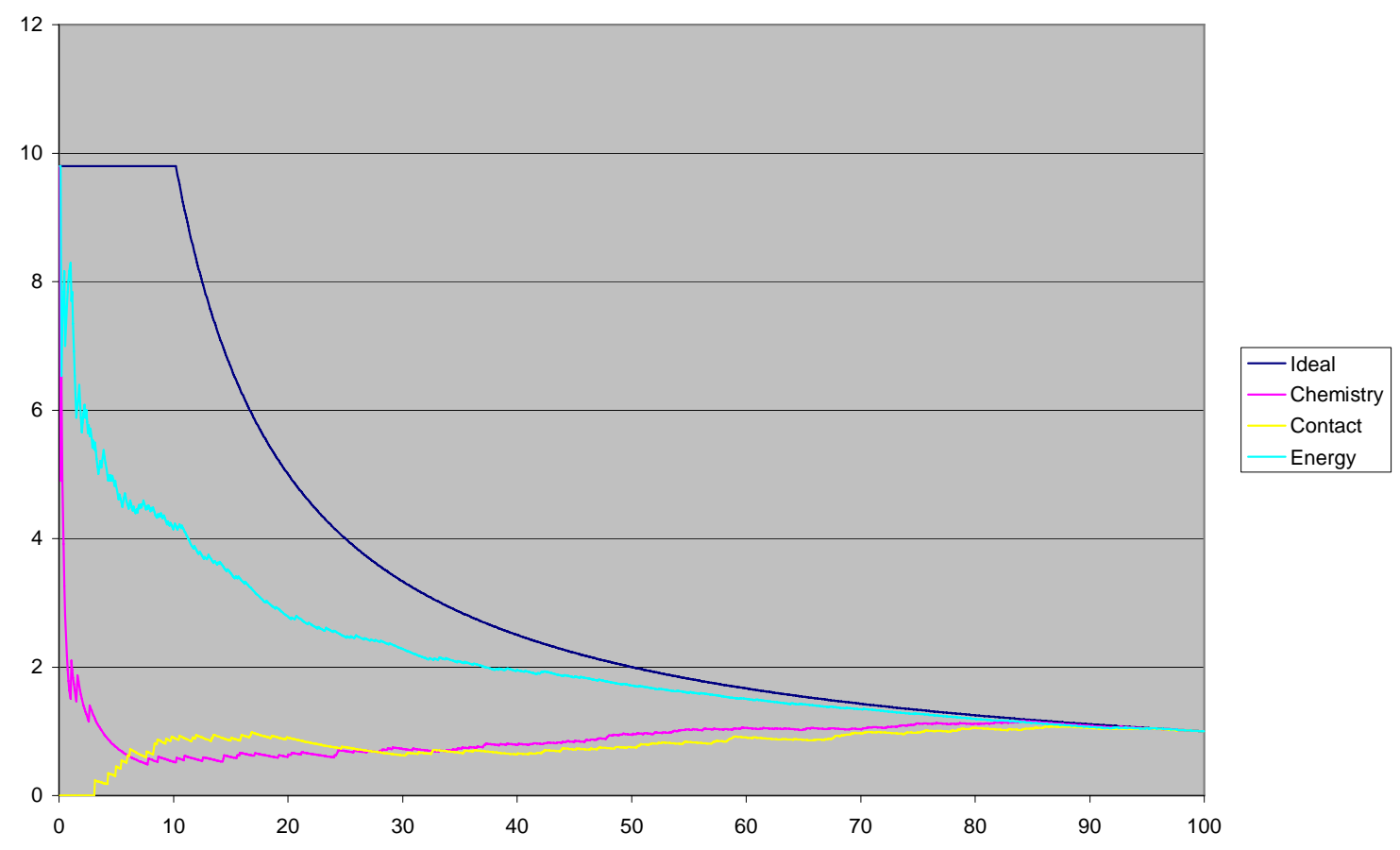




\section{Dockit}

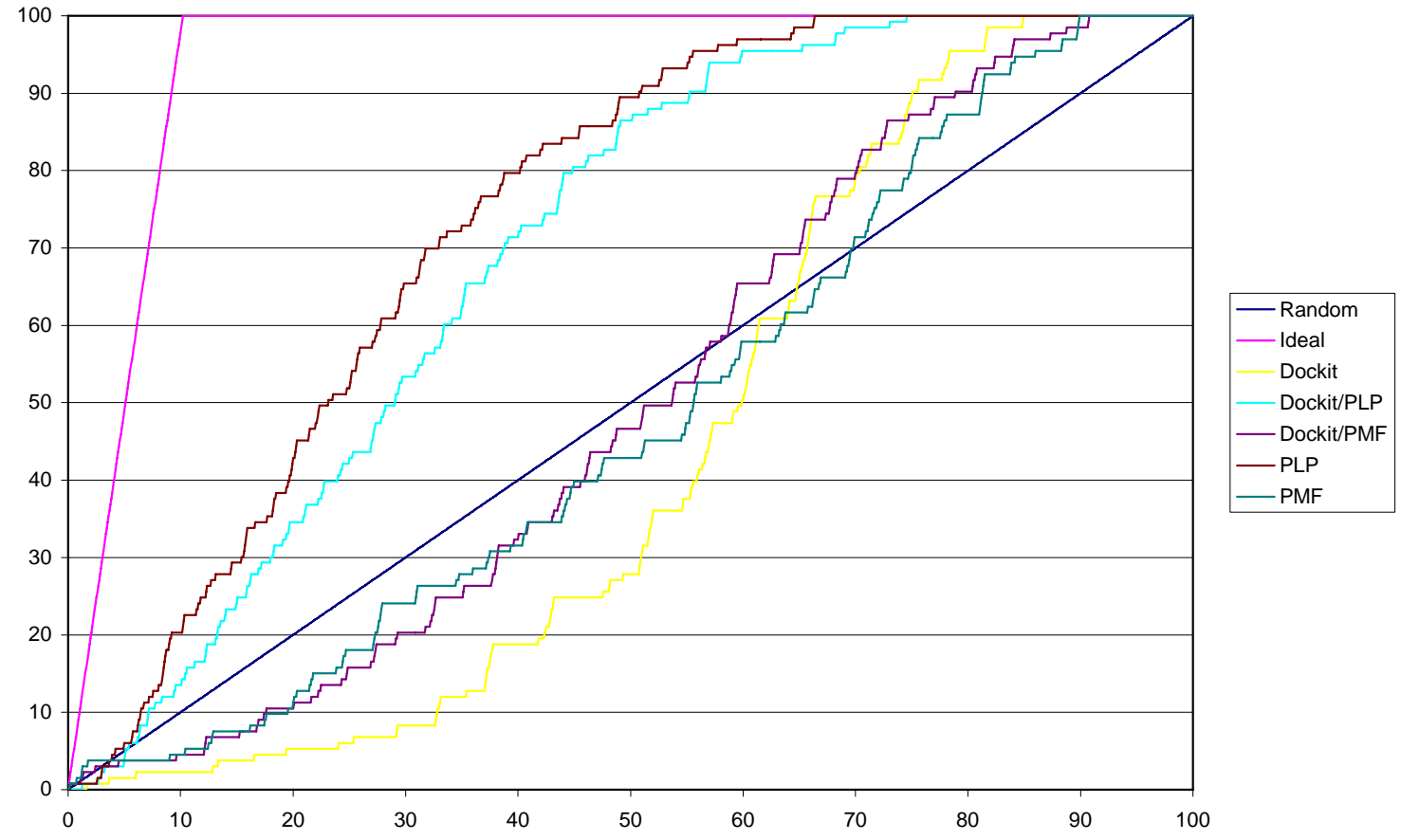

Dockit

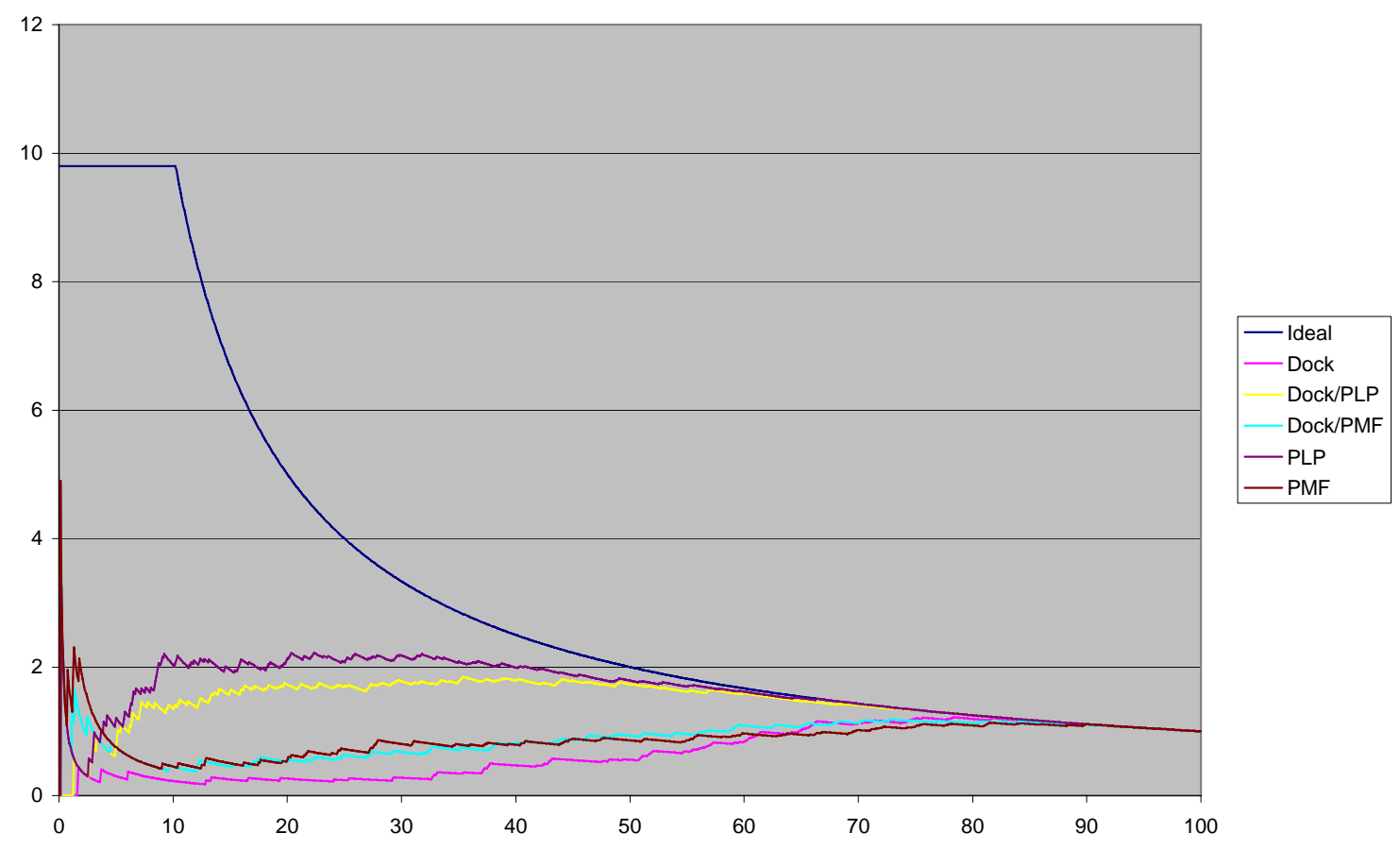


FlexX

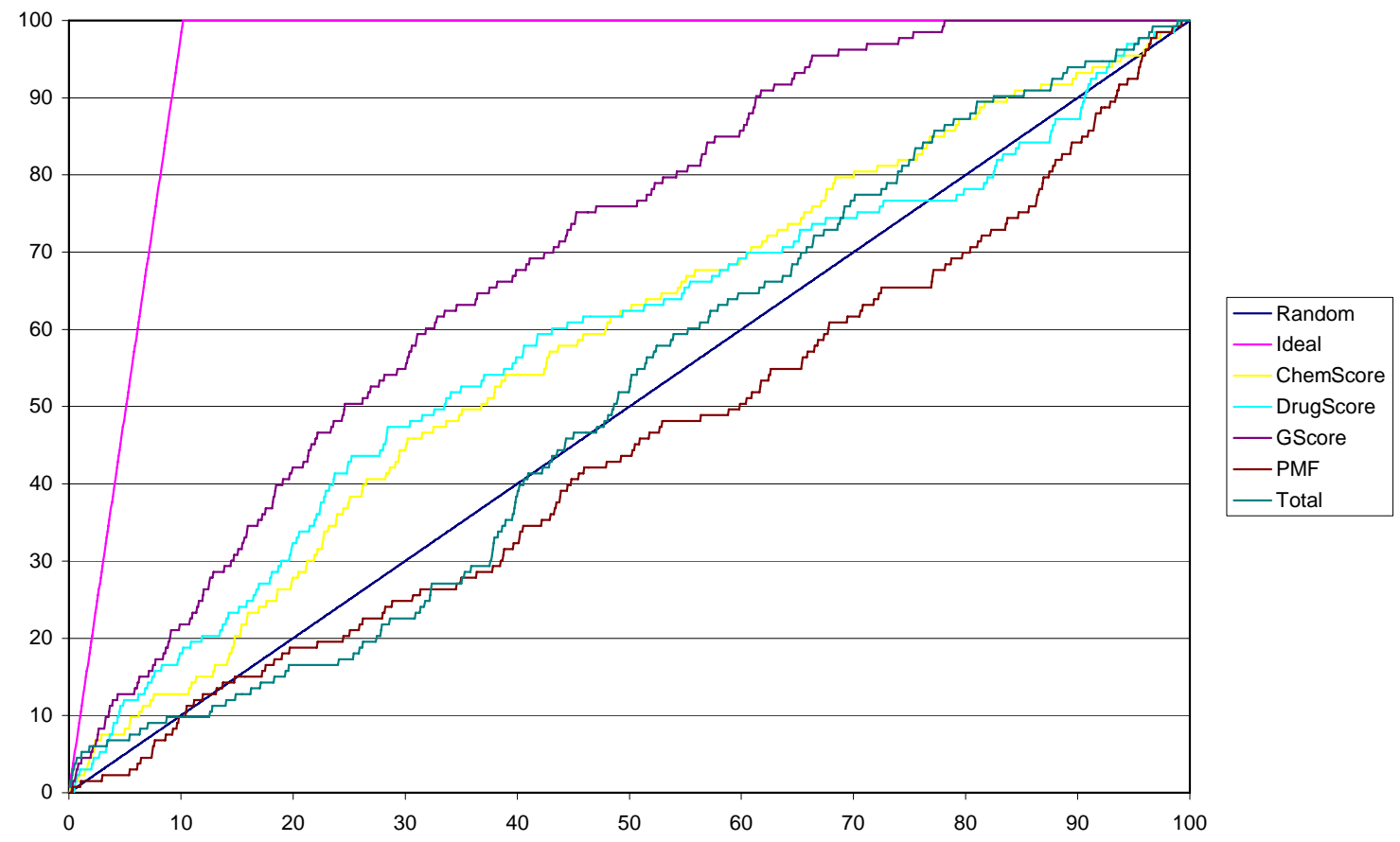

FlexX

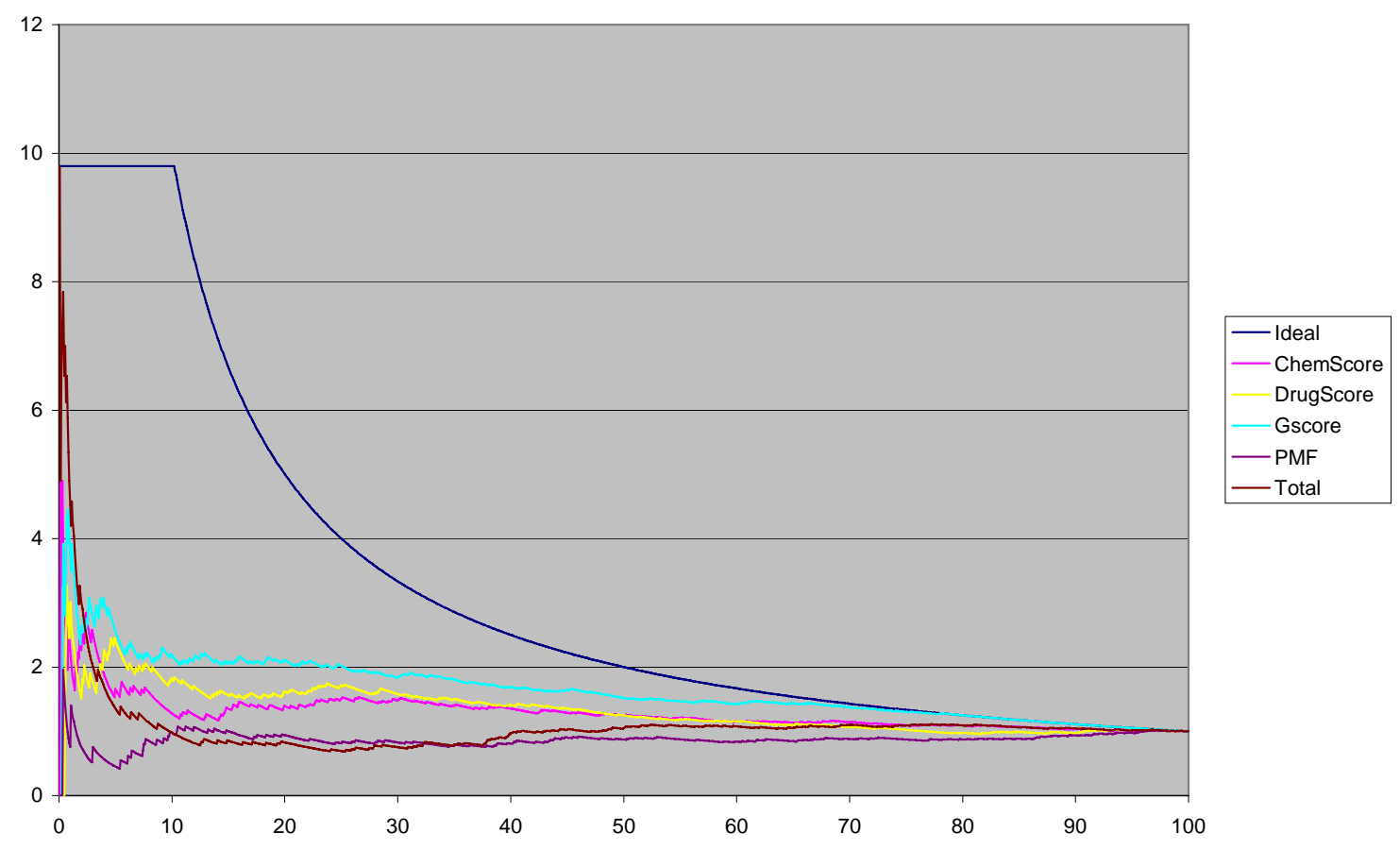


Flo

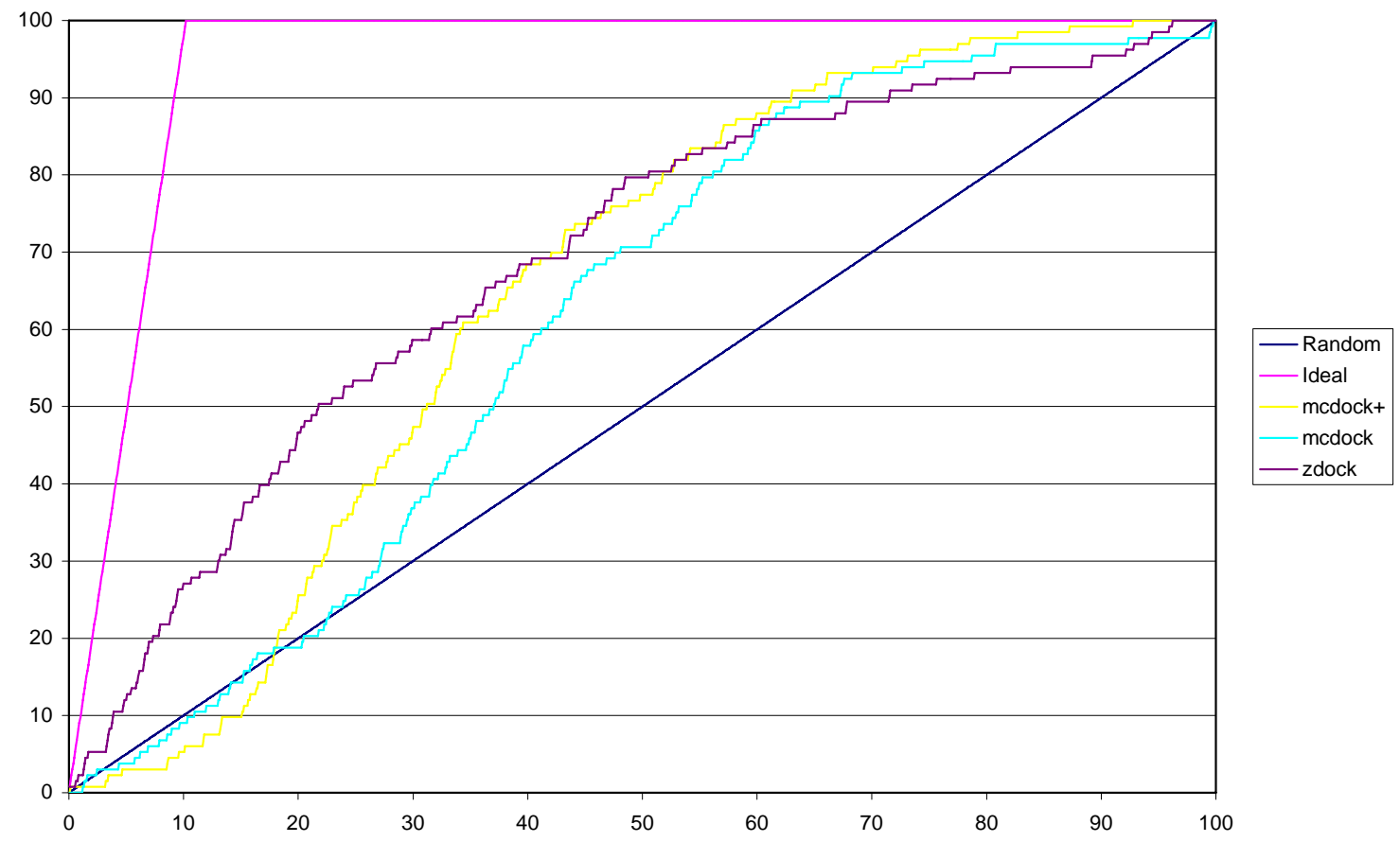

Flo

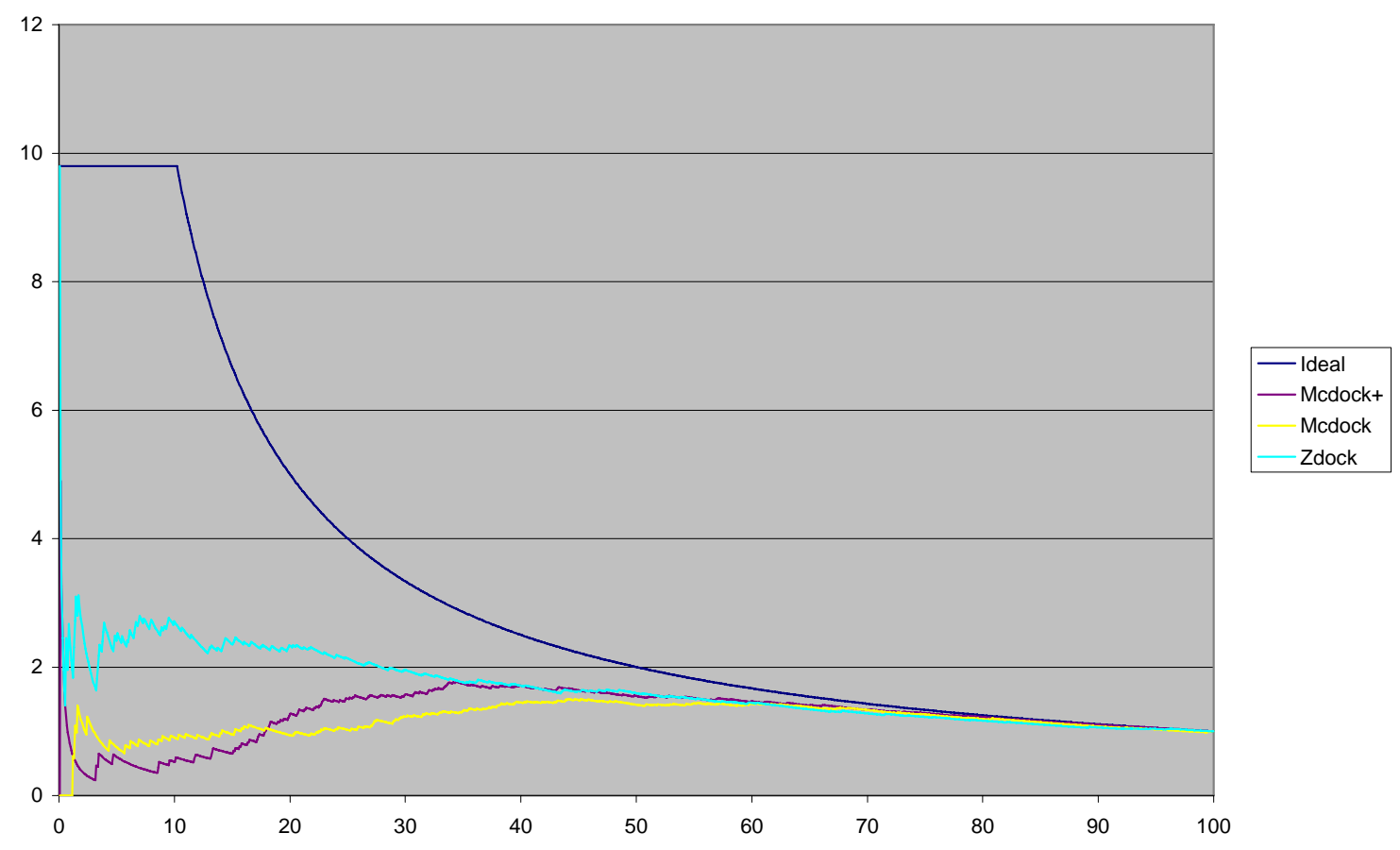


Fred

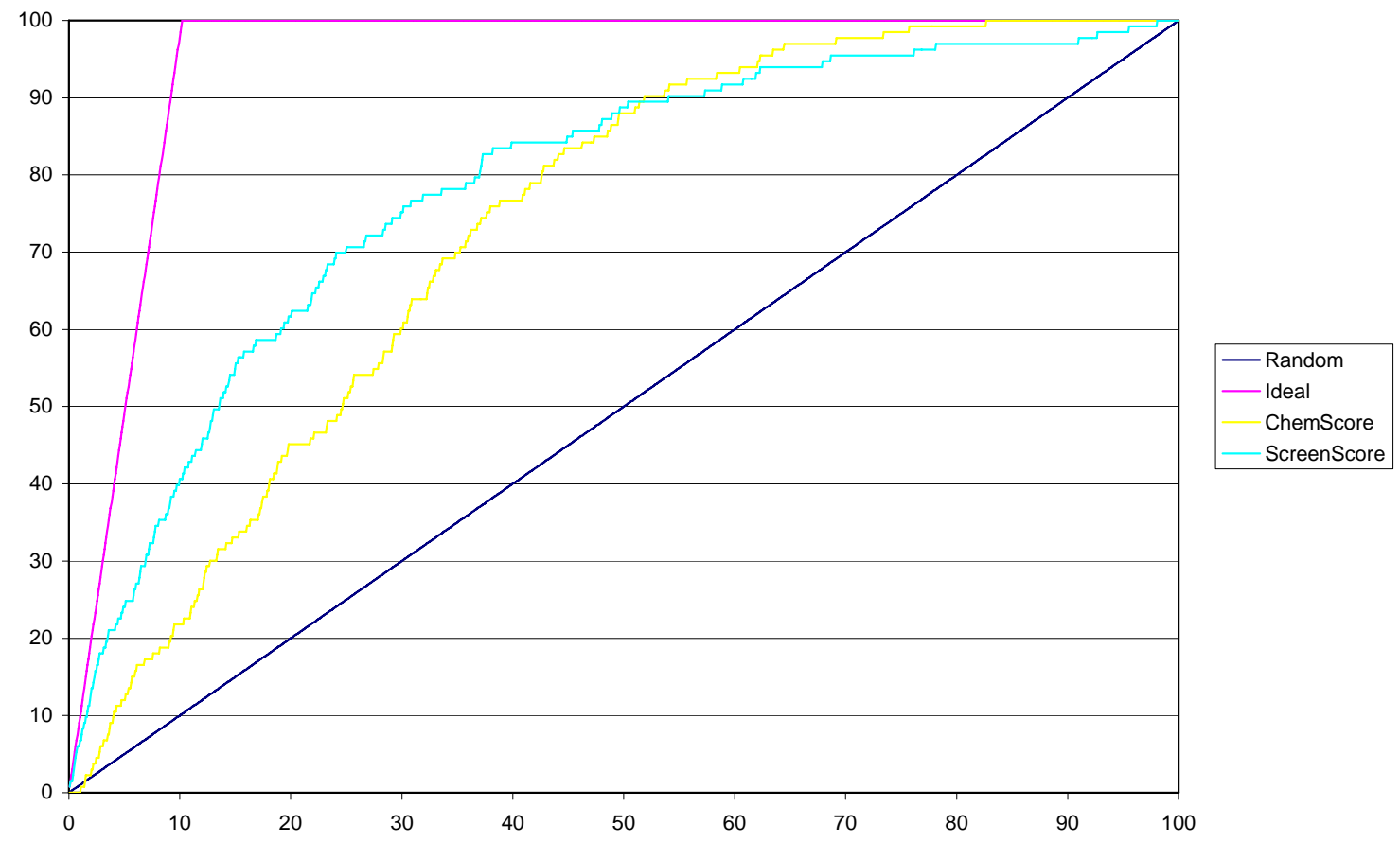

Fred

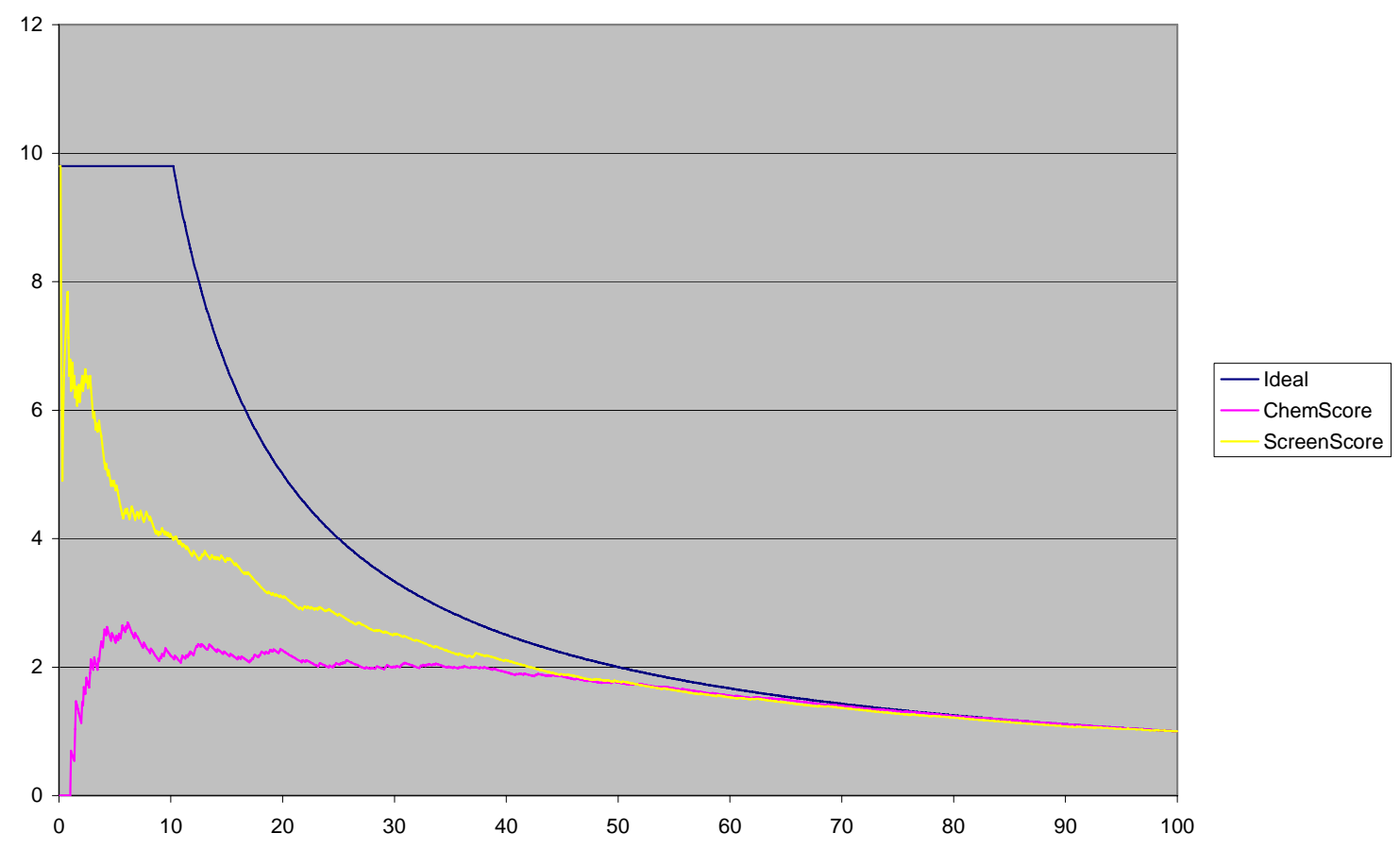




\section{Glide}

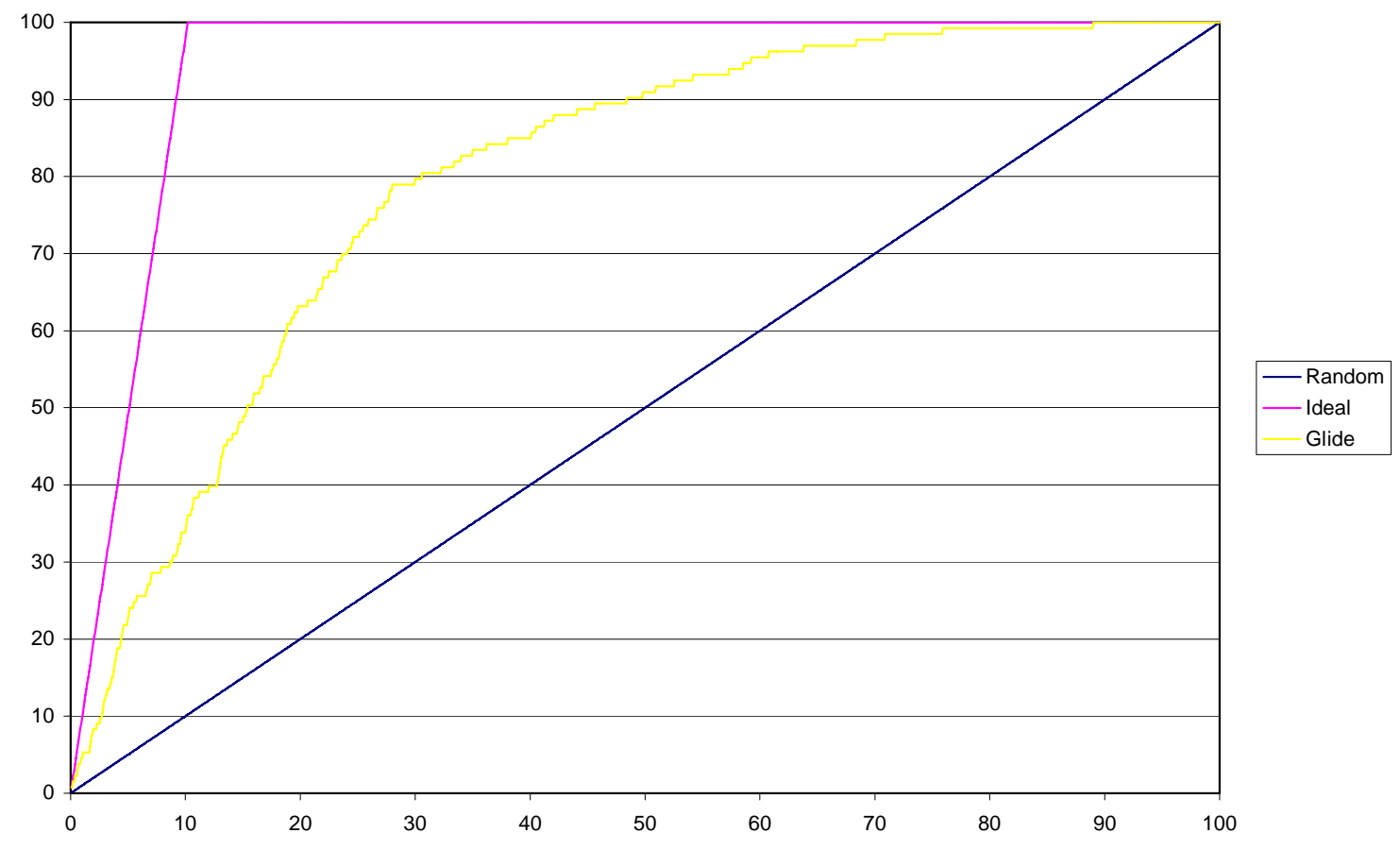

Glide

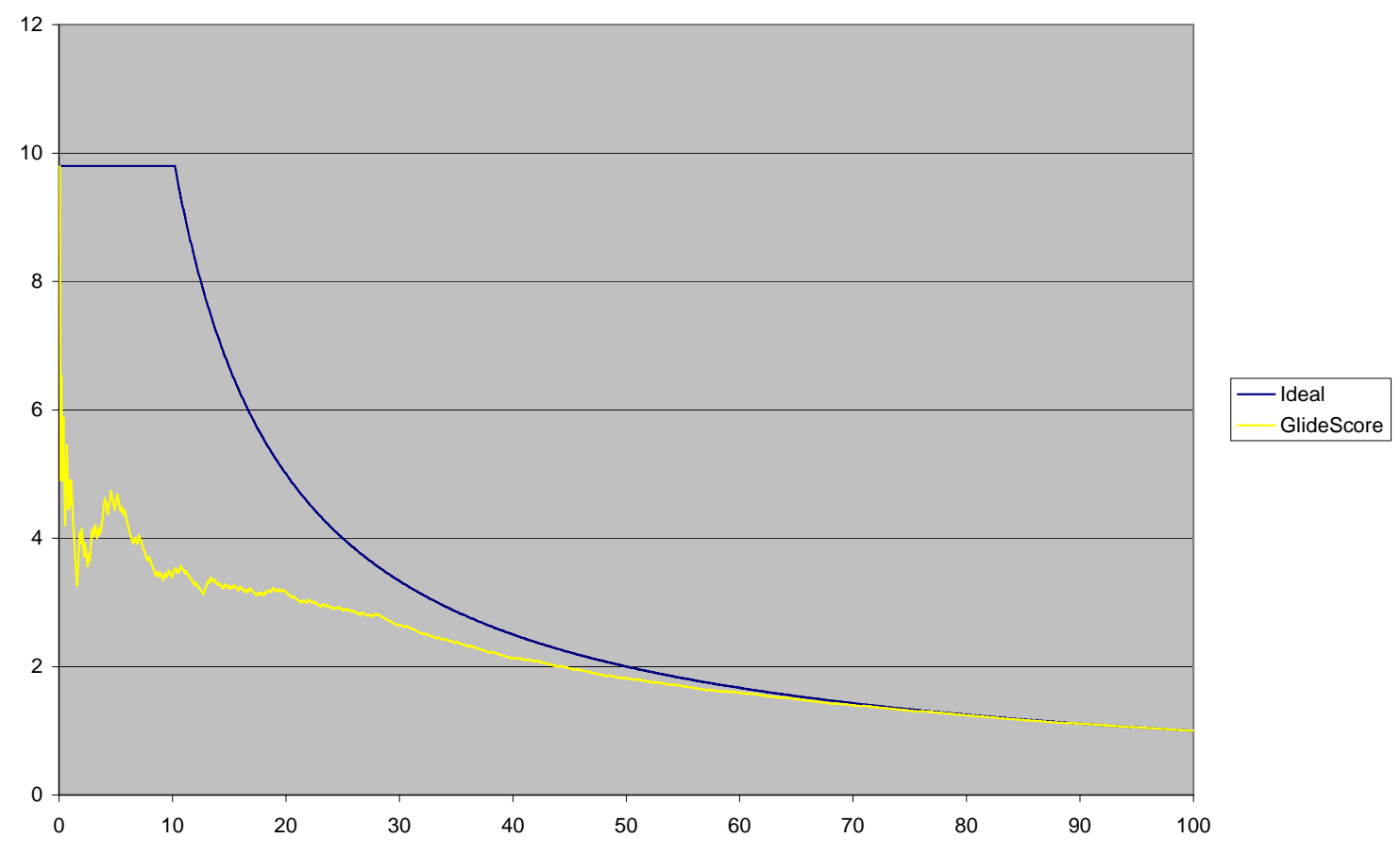


Gold

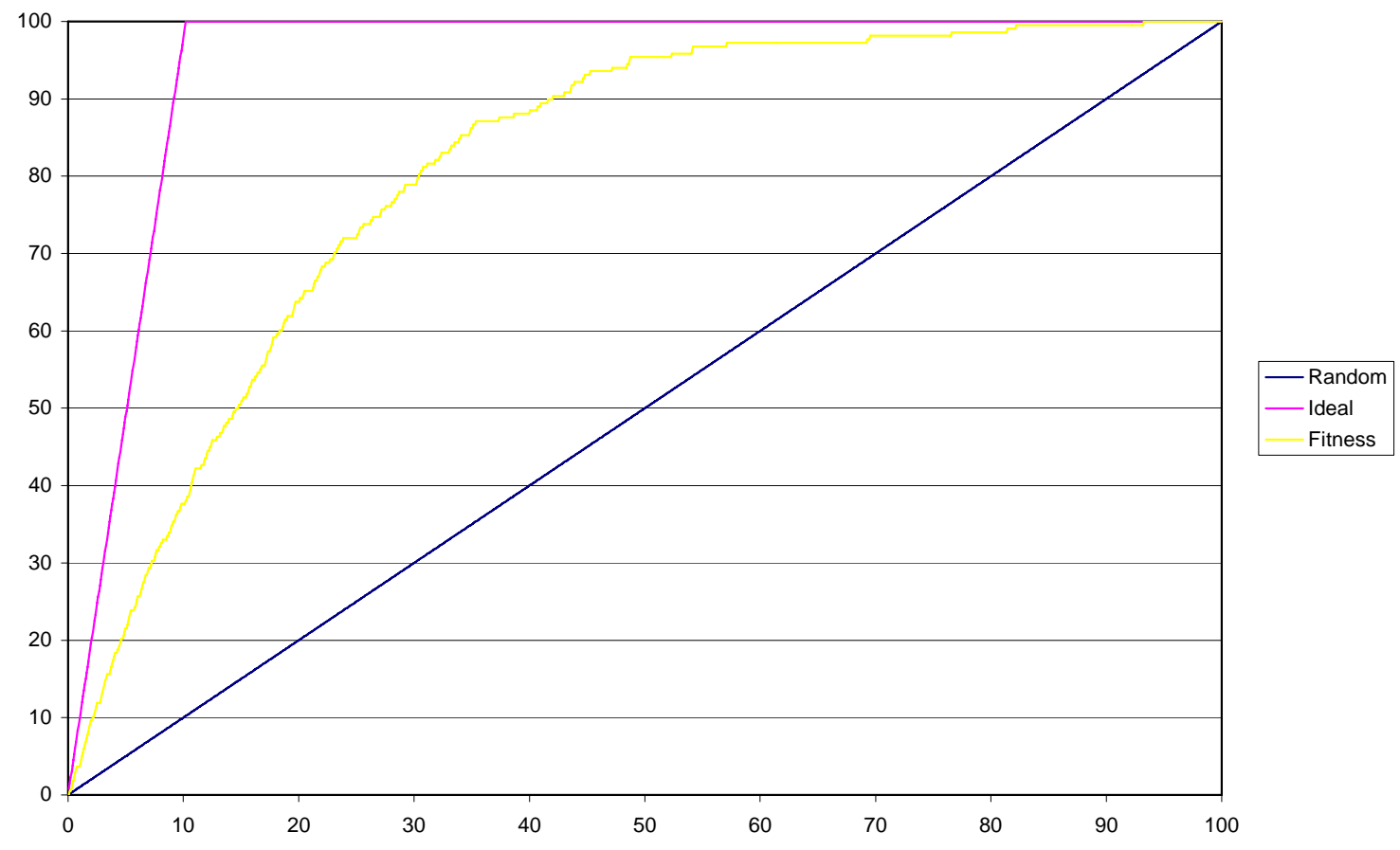

Gold

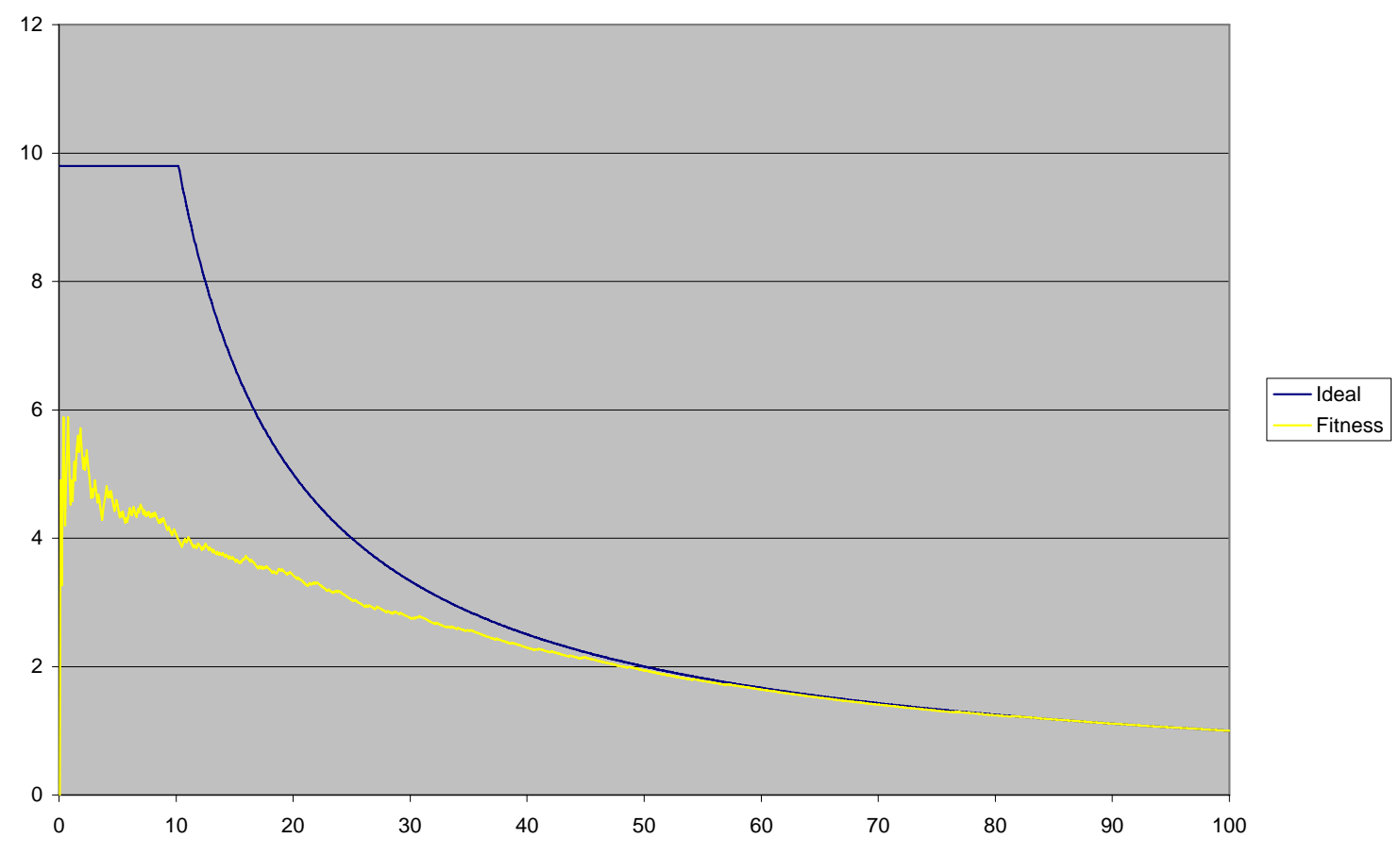


LigFit

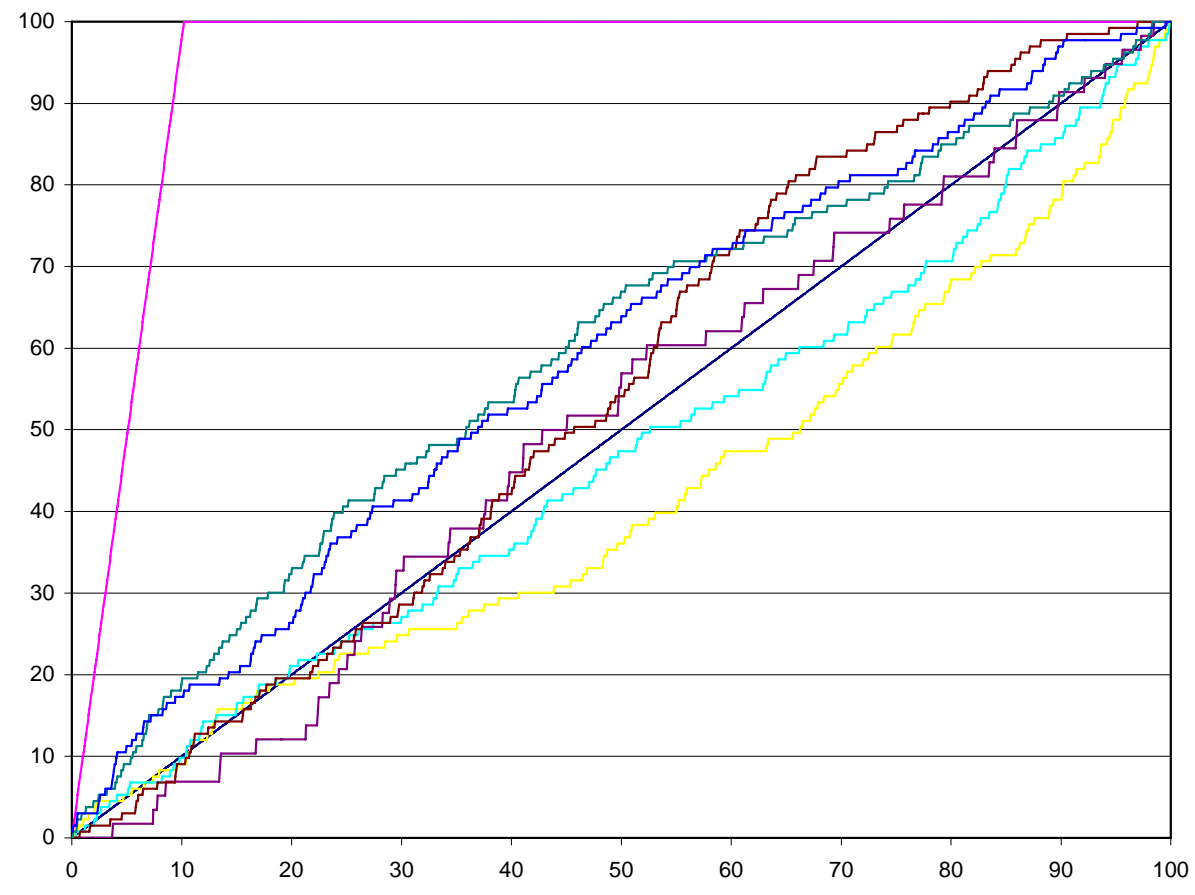

\begin{tabular}{|l|}
\hline - Random \\
- Ideal \\
DrugScore, CFF \\
- DrugScore, Dreiding \\
- LigScore2, CFF \\
- LigScore2, Dreiding \\
- PMF, CFF \\
- PMF, Dreiding \\
\hline
\end{tabular}

LigFit

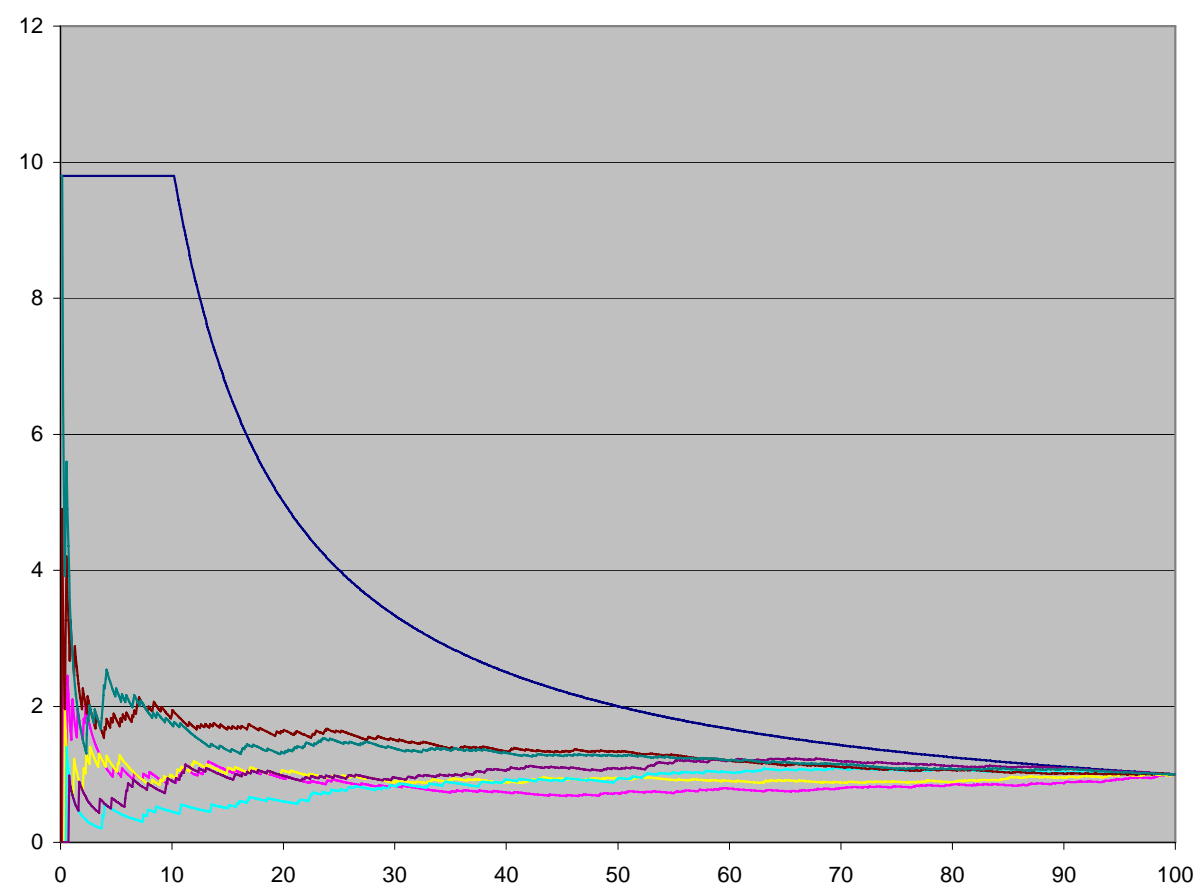

- Ligscore2, Dreiding - PMF, CFF

- PMF, Dreiding 
MOE

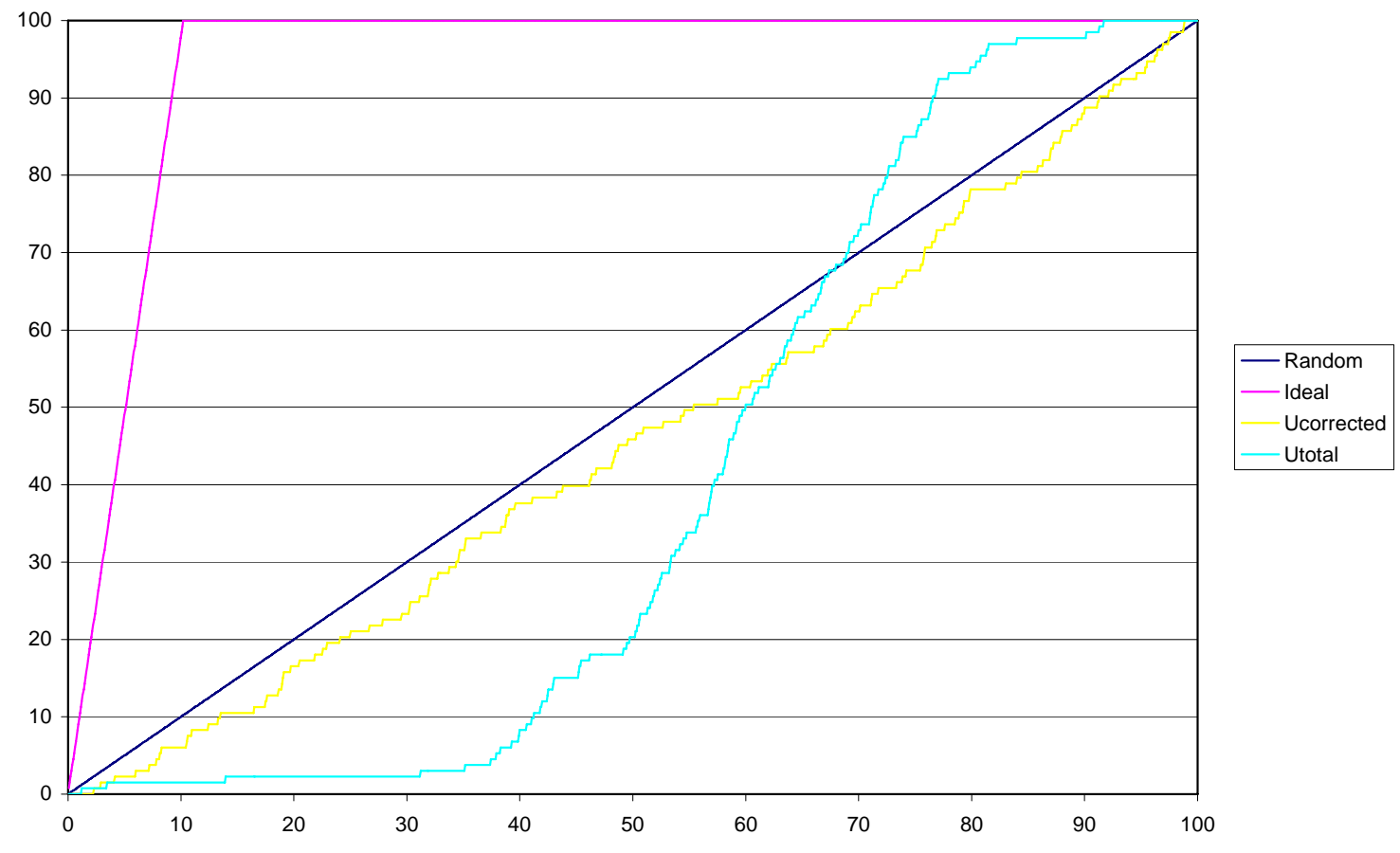

MOE

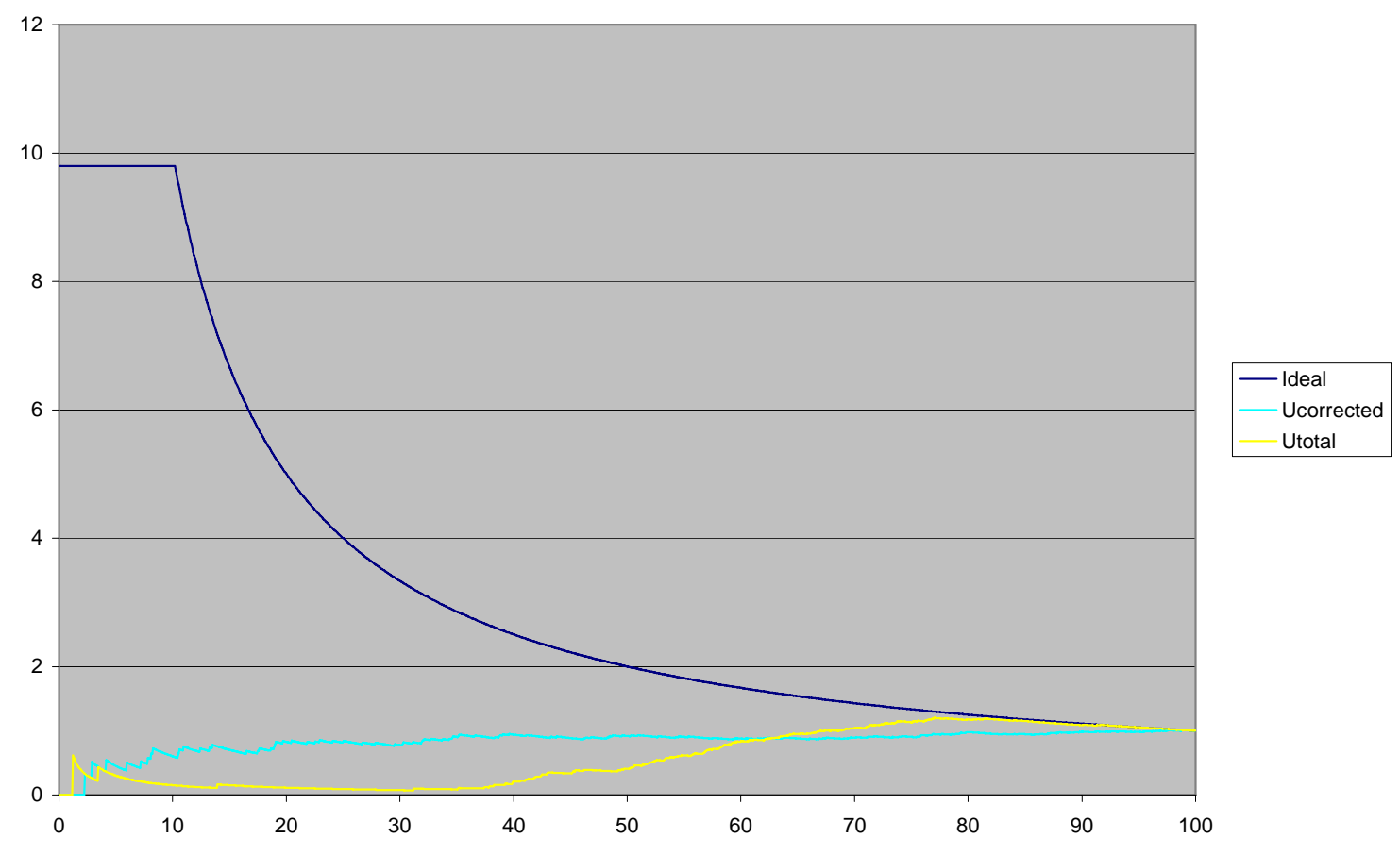


MVP

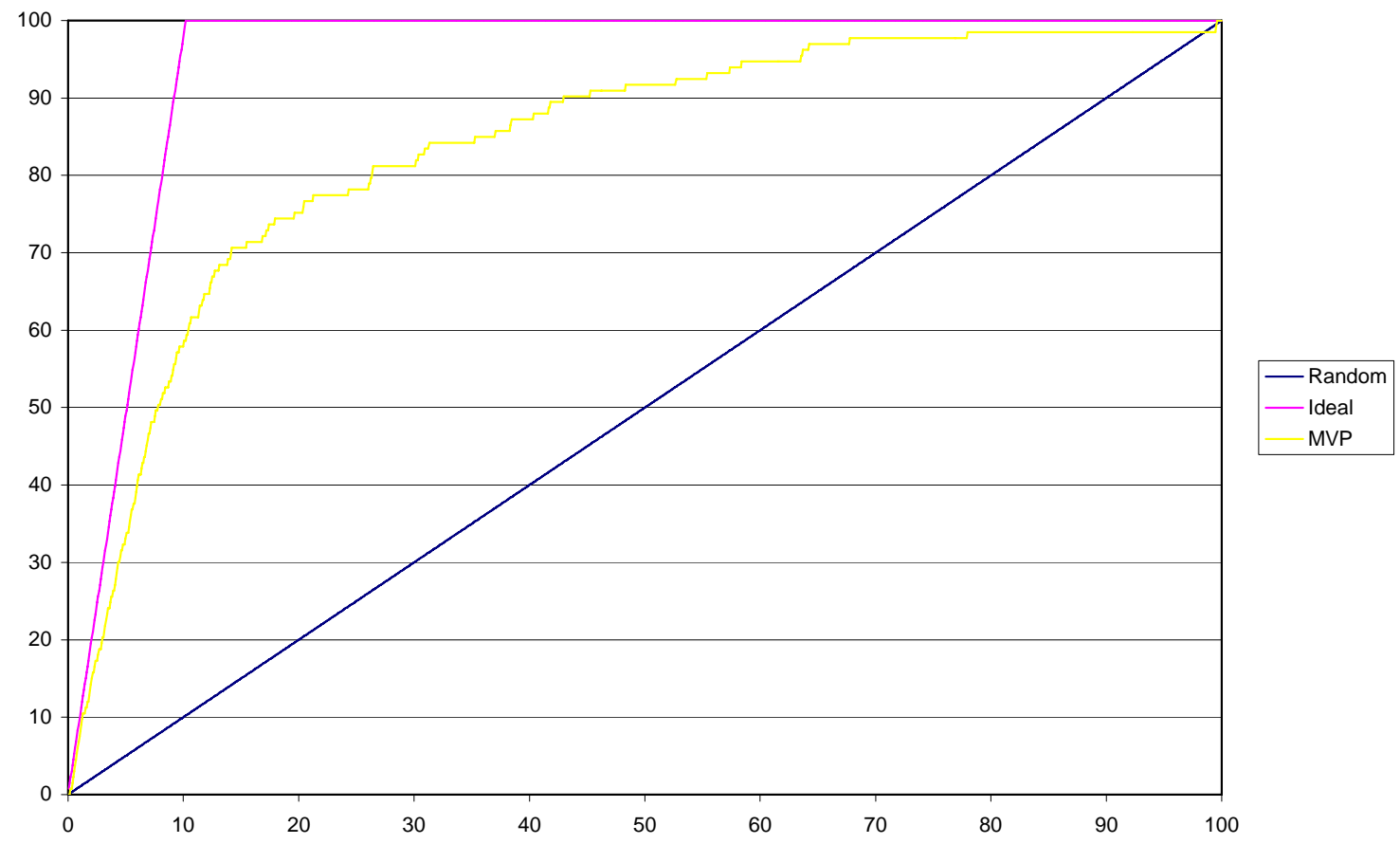

MVP

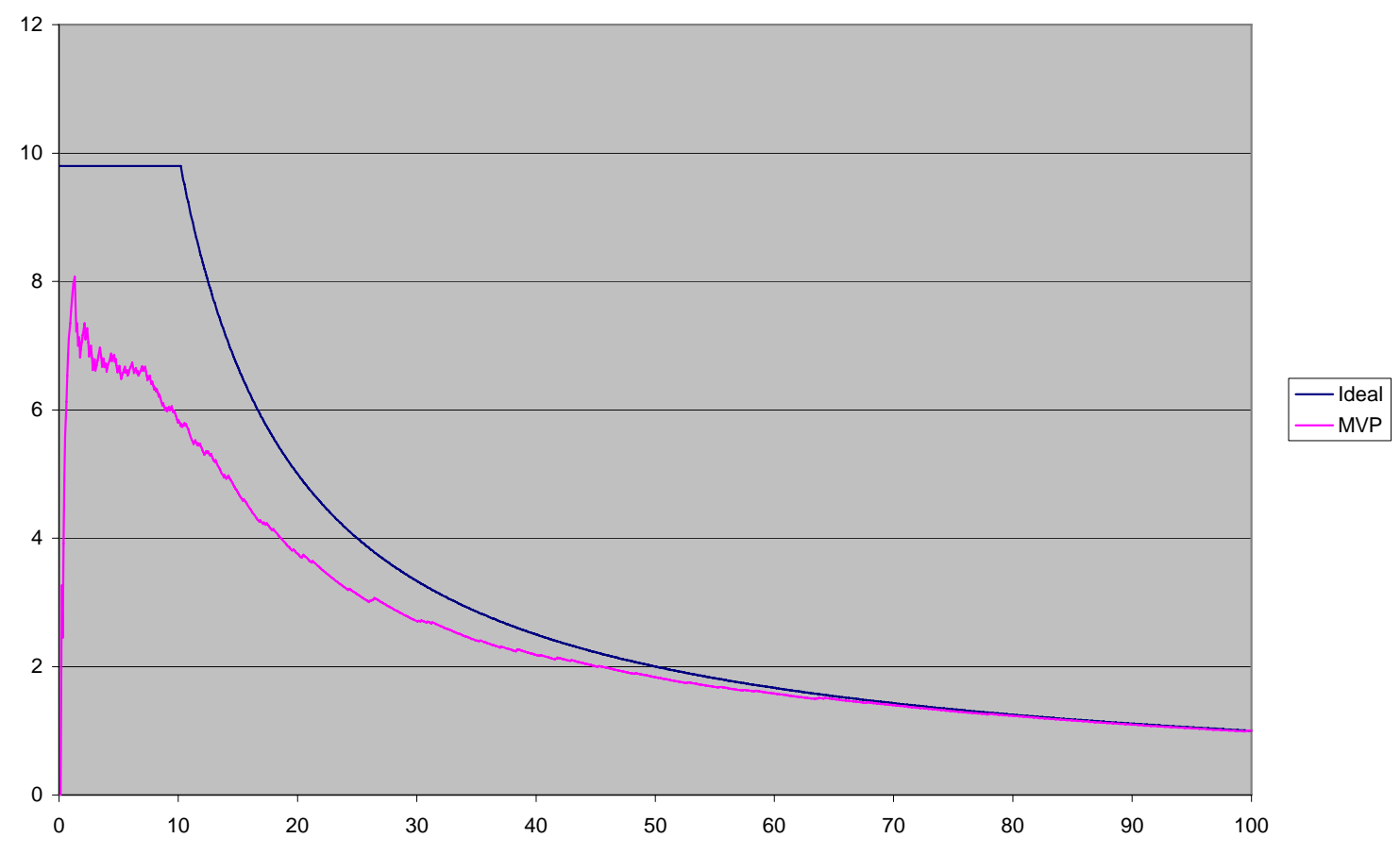


Dock4

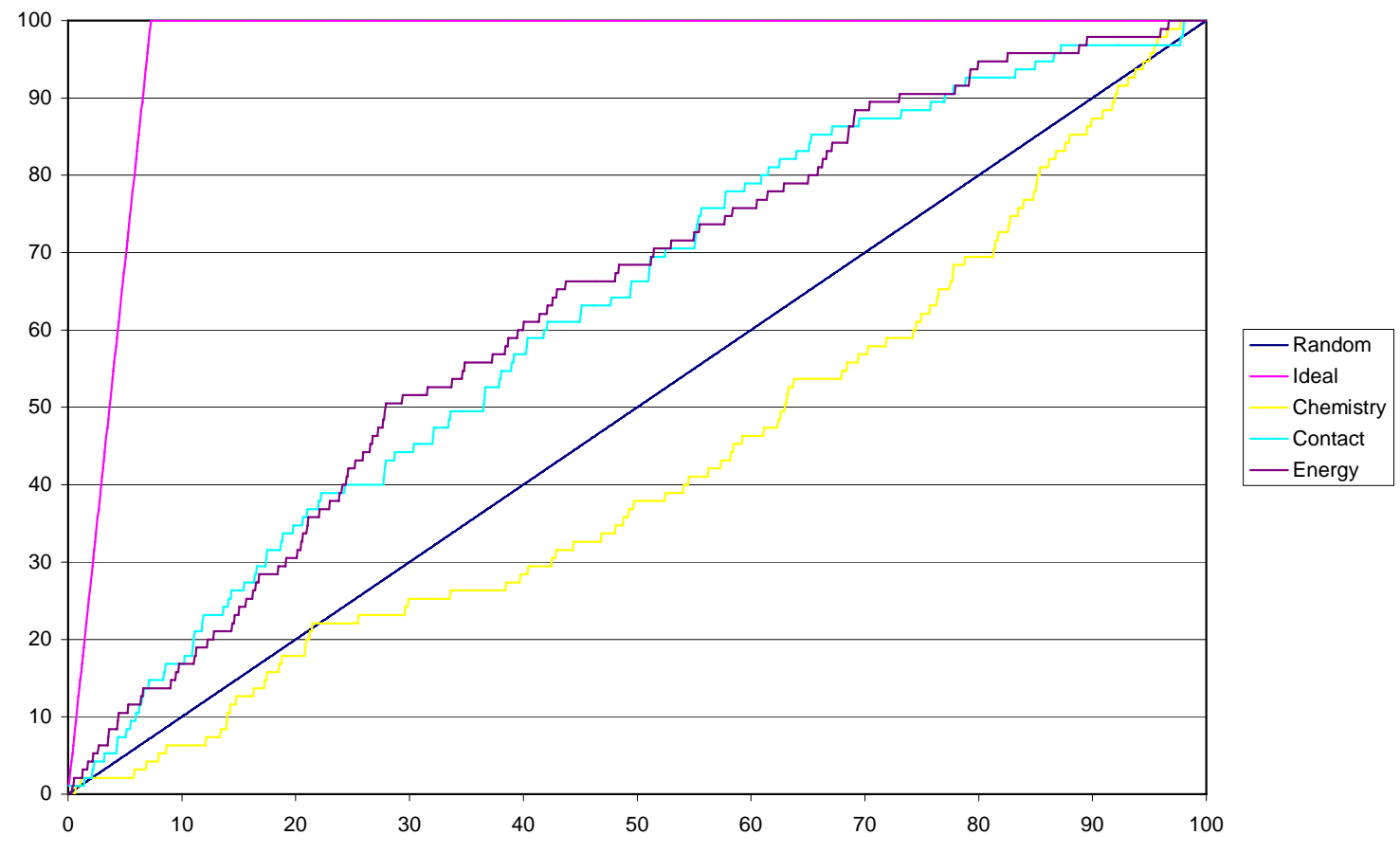

Dock4

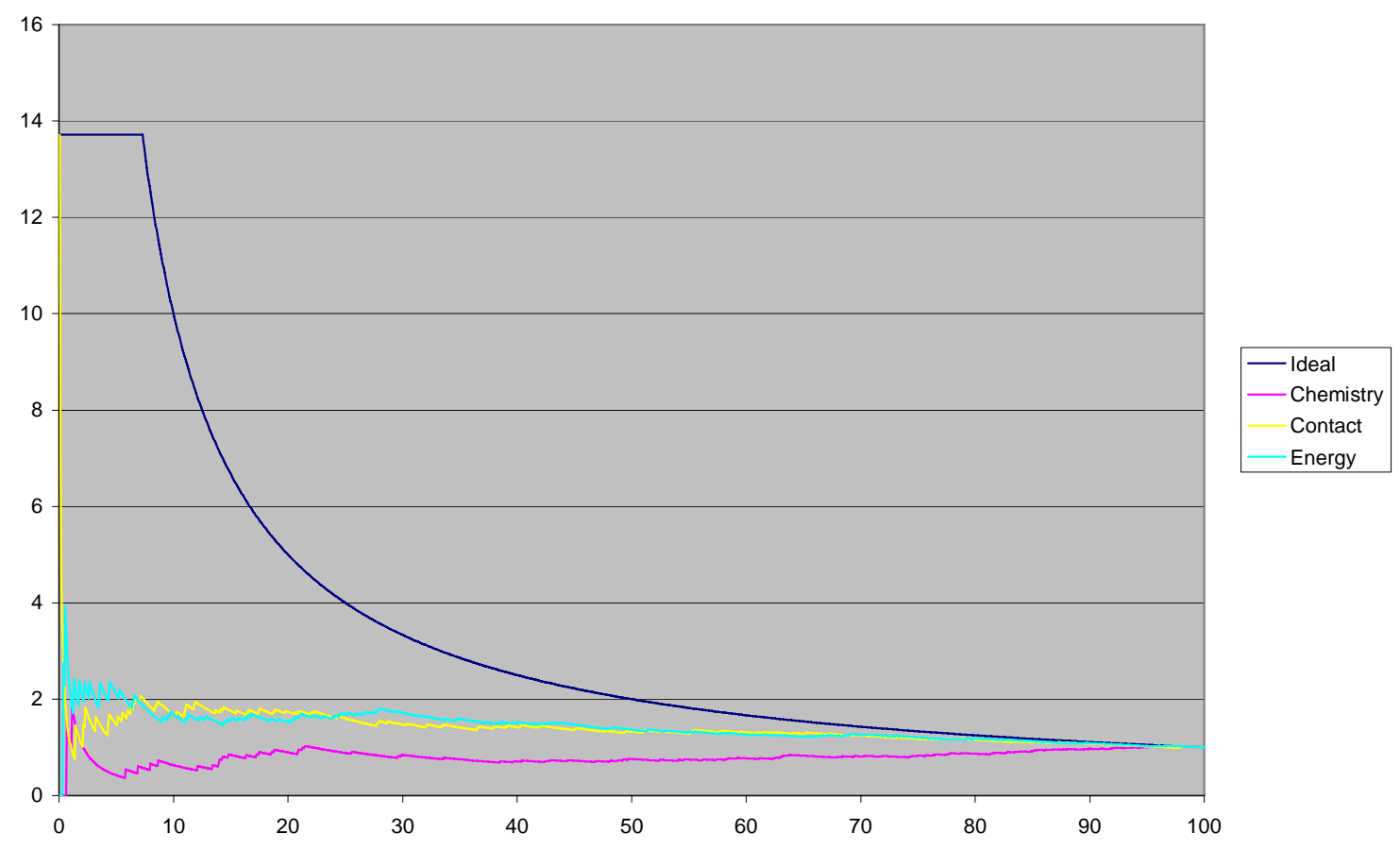




\section{Dockit}

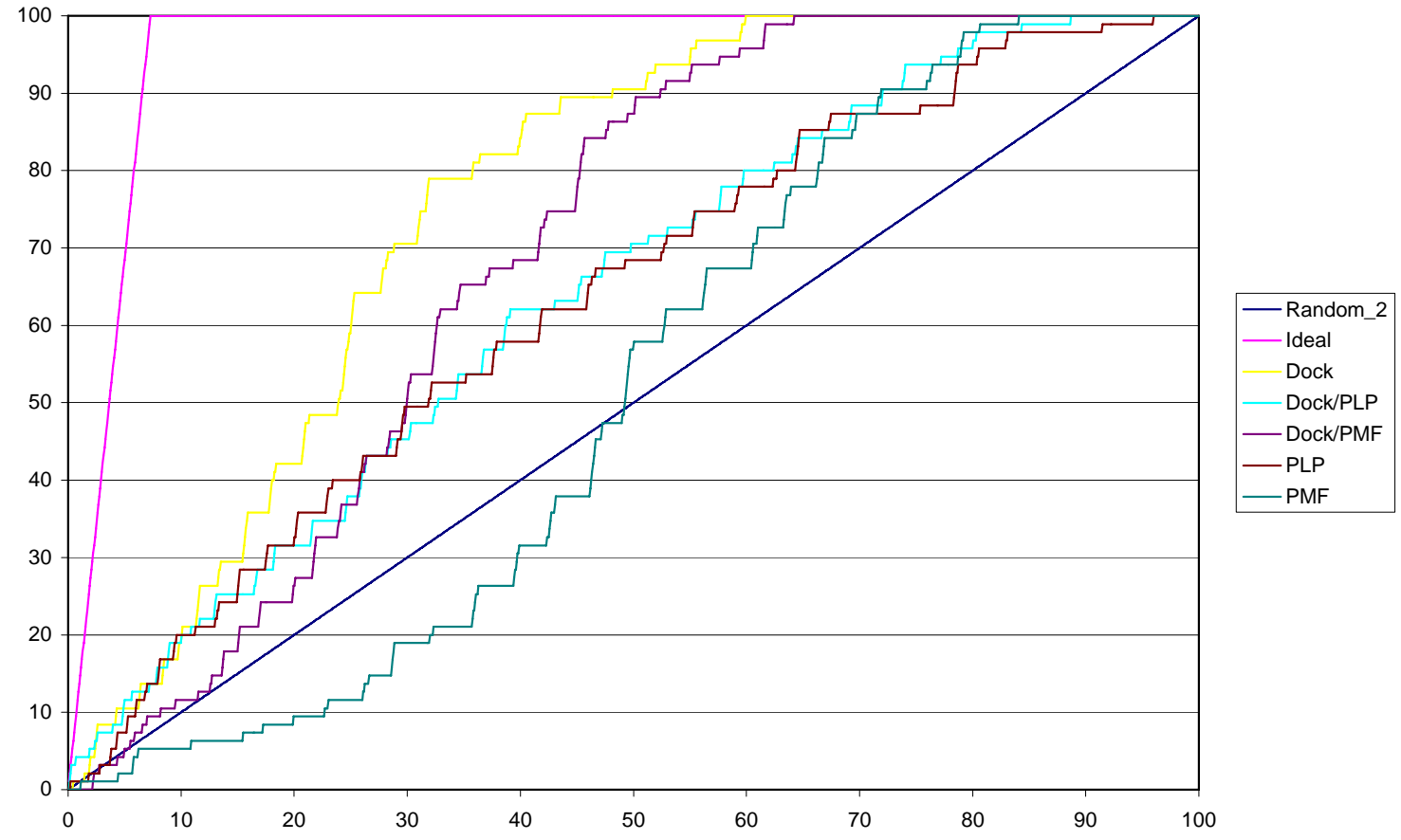

Dockit

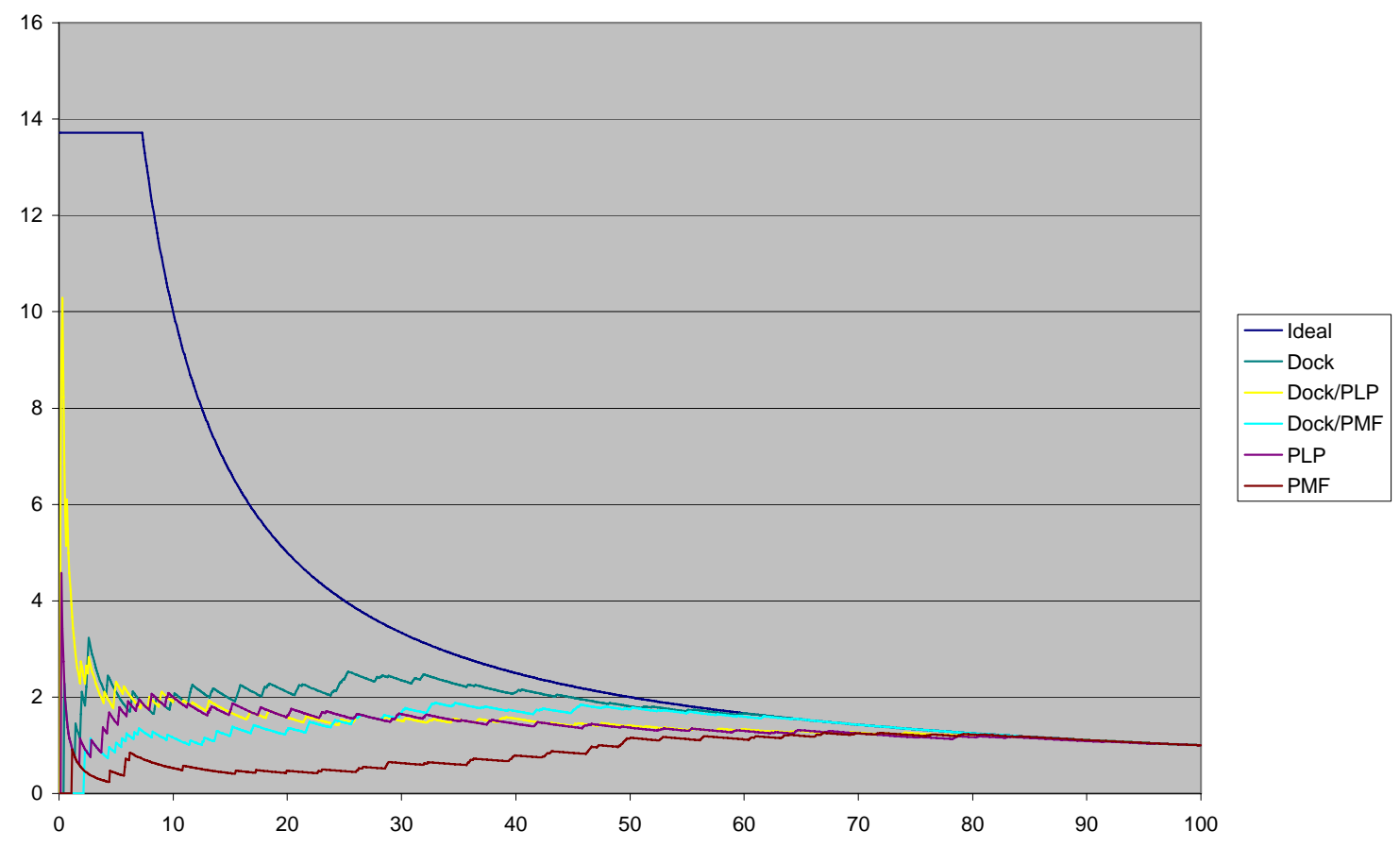


FlexX

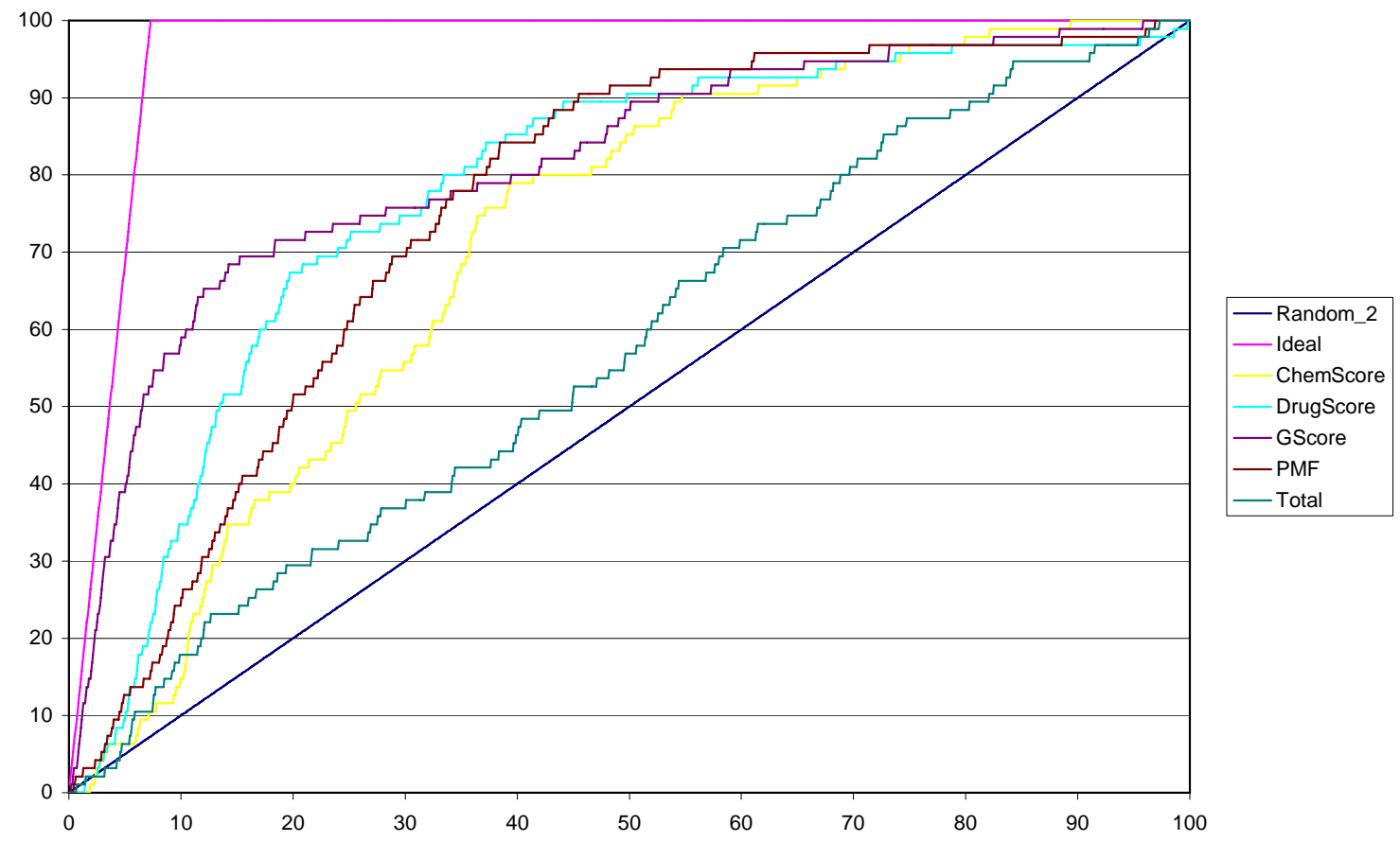

FlexX

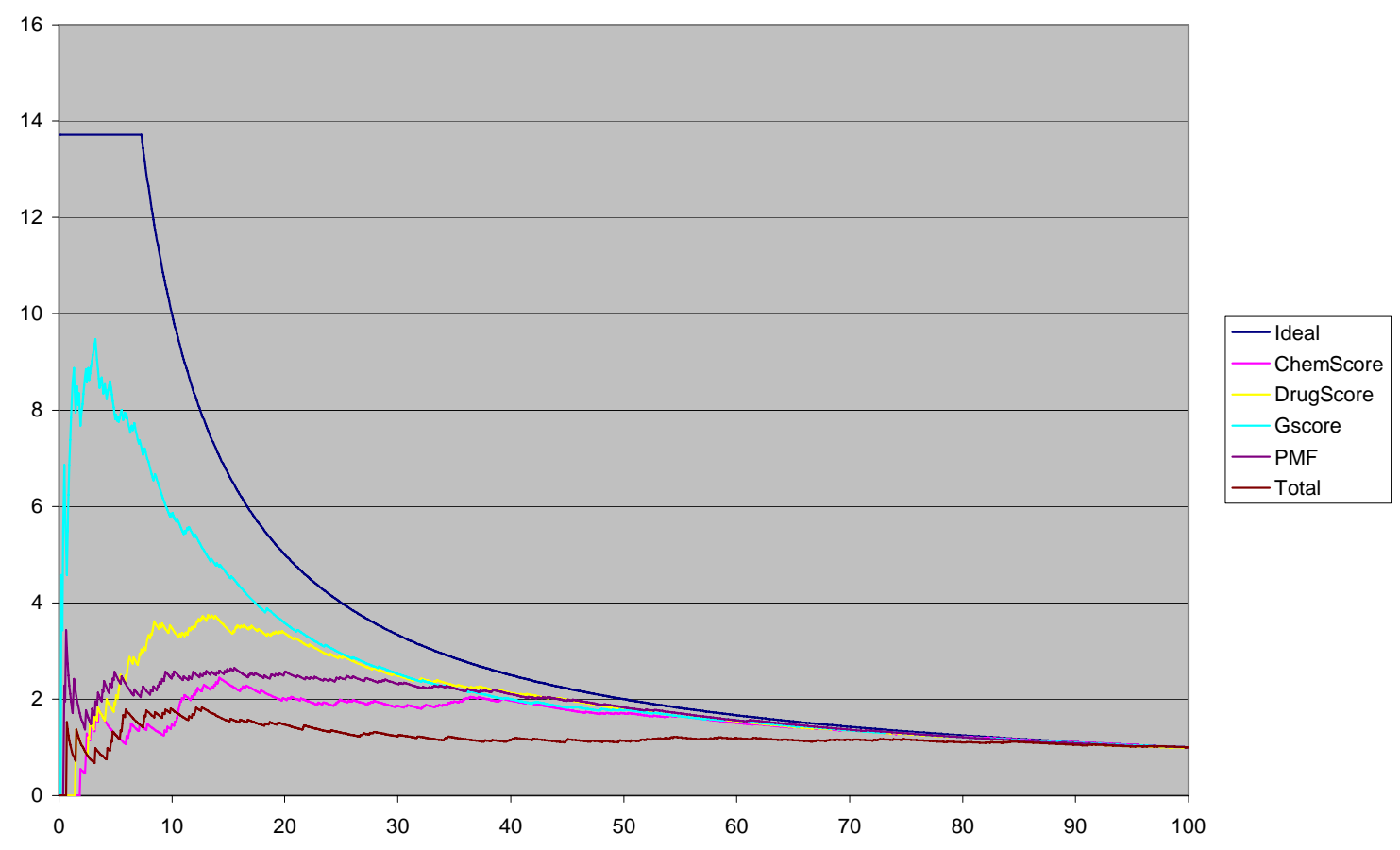




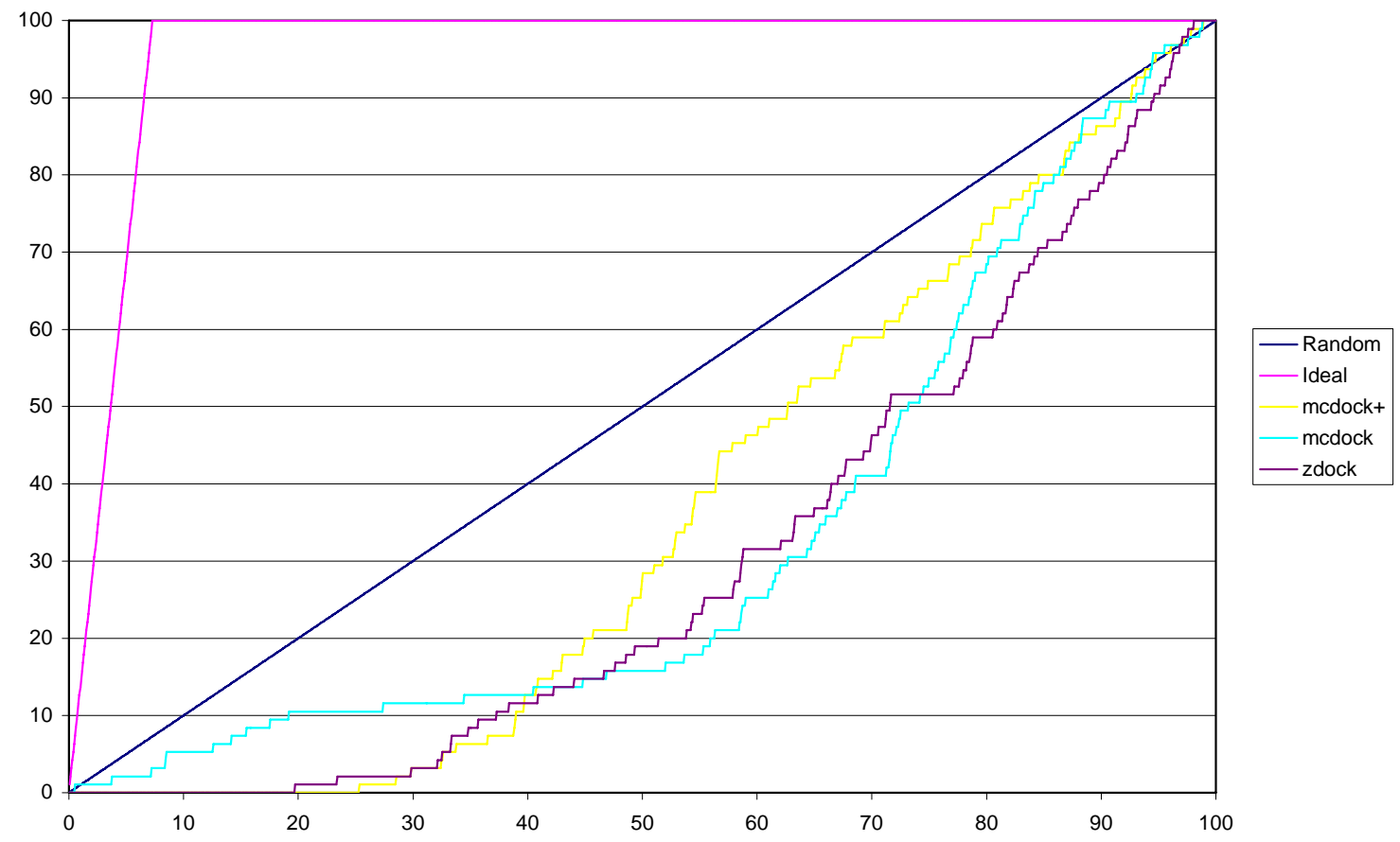

Flo

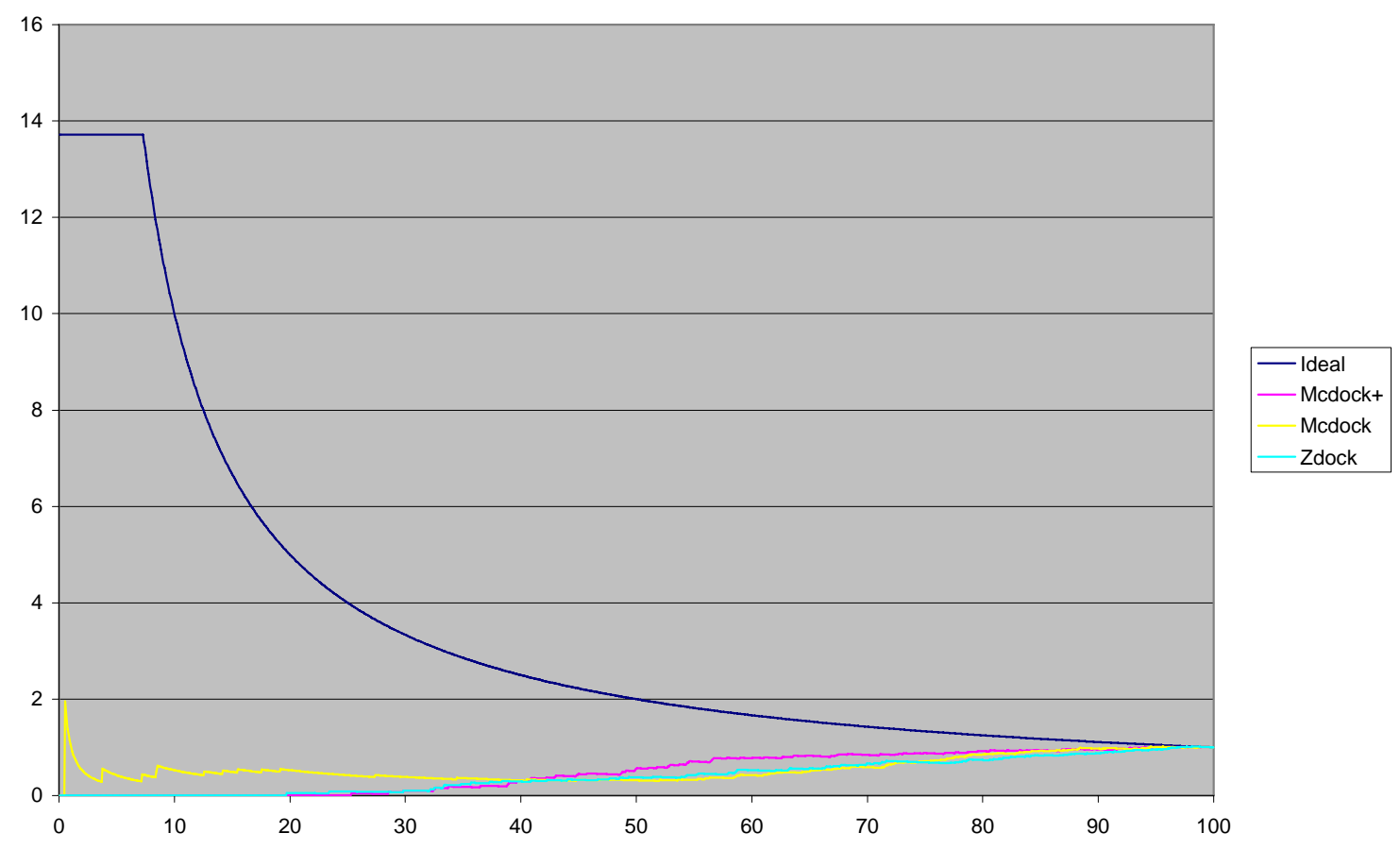


Fred

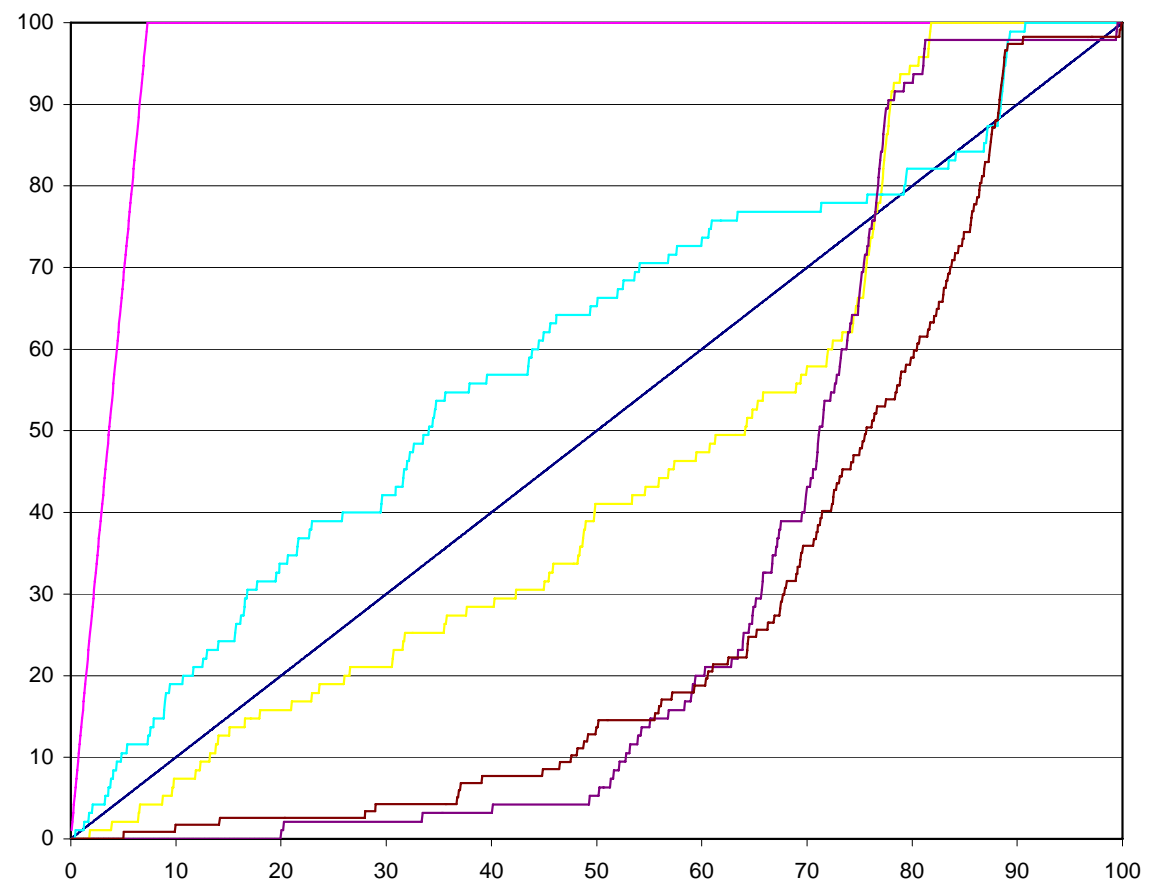

\begin{tabular}{|l|}
\hline - Random \\
- Ideal \\
ChemScore \\
C ChemScore, loop model \\
- ScreenScore \\
- ScreenScore, loop model \\
\hline
\end{tabular}

Fred

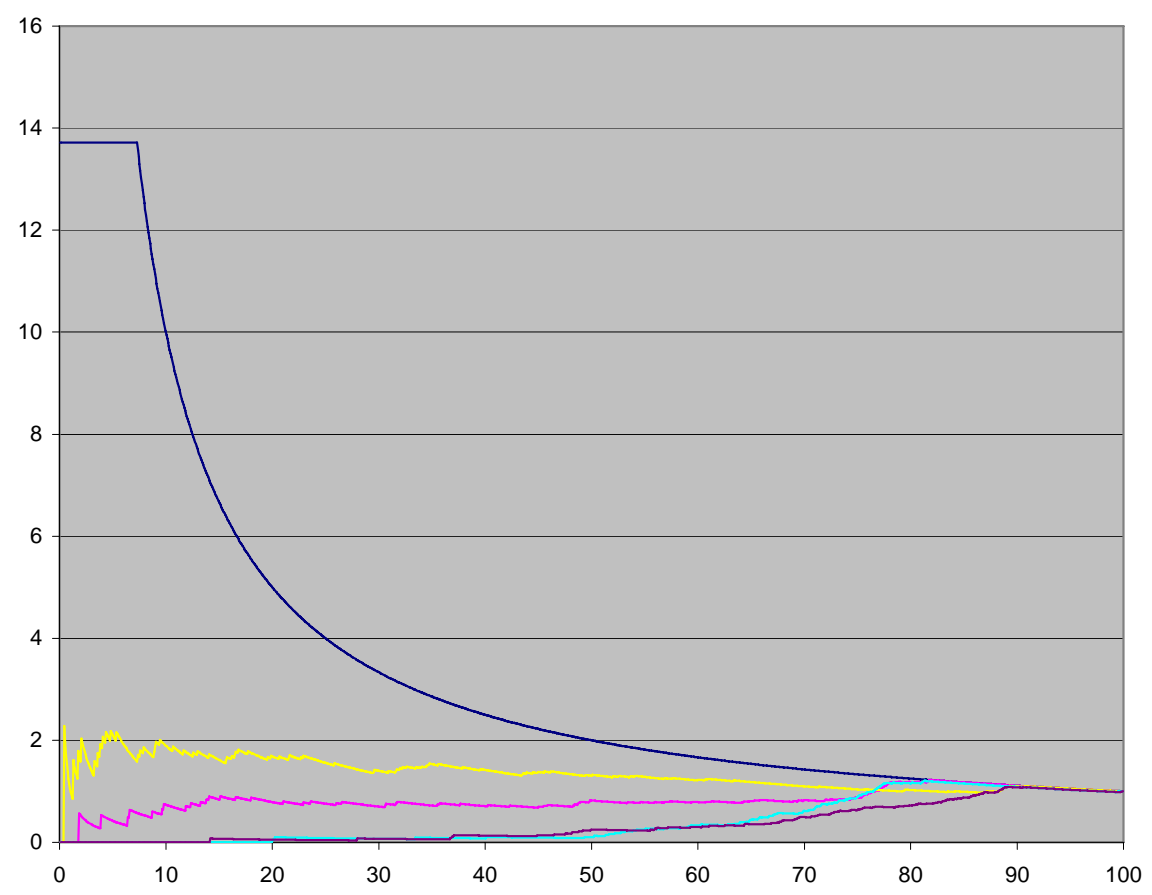

- Ideal
- ChemScore
ChemScore, loop model
- ScreenScore
- ScreenScore, loop model




\section{Glide}

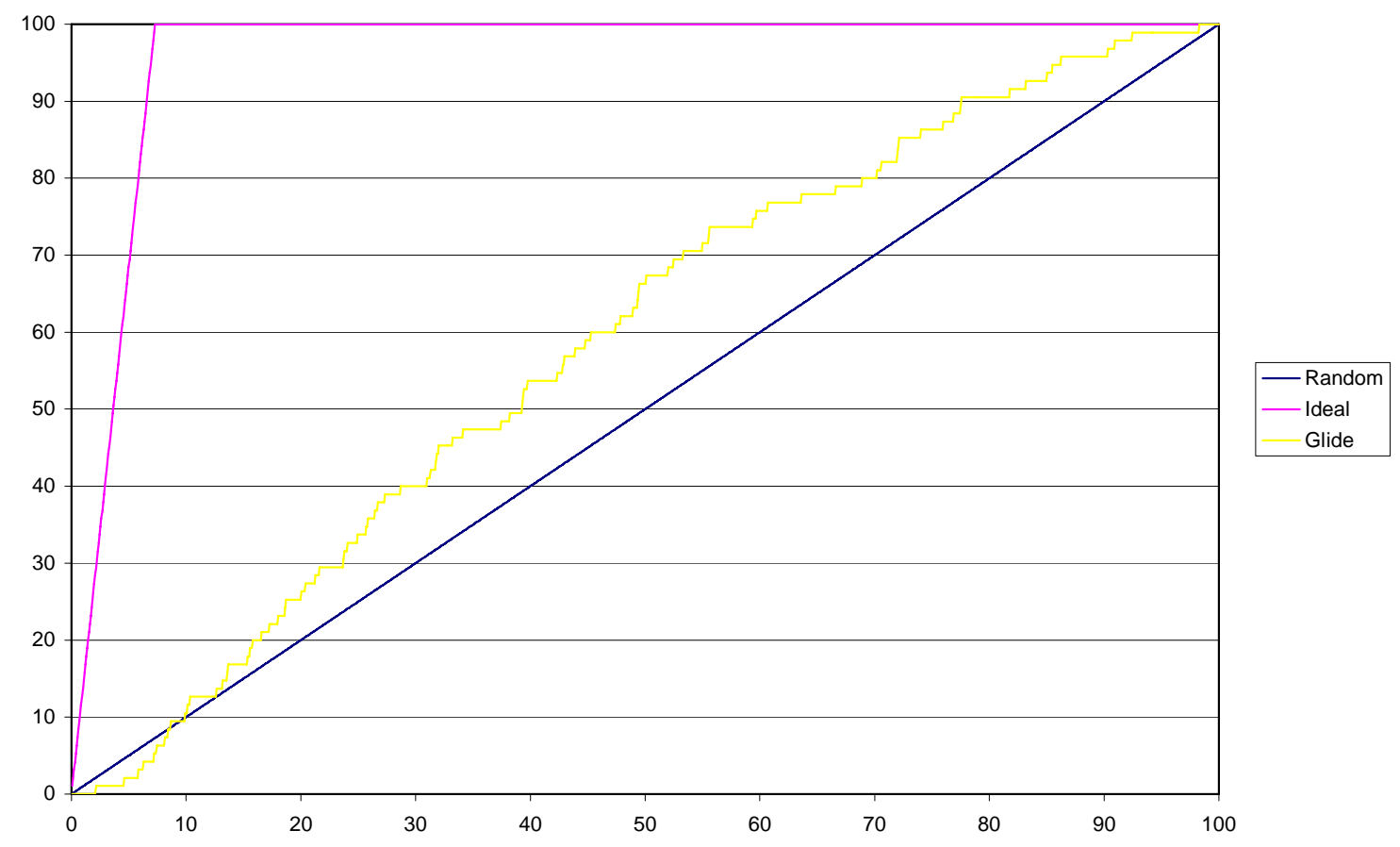

Glide

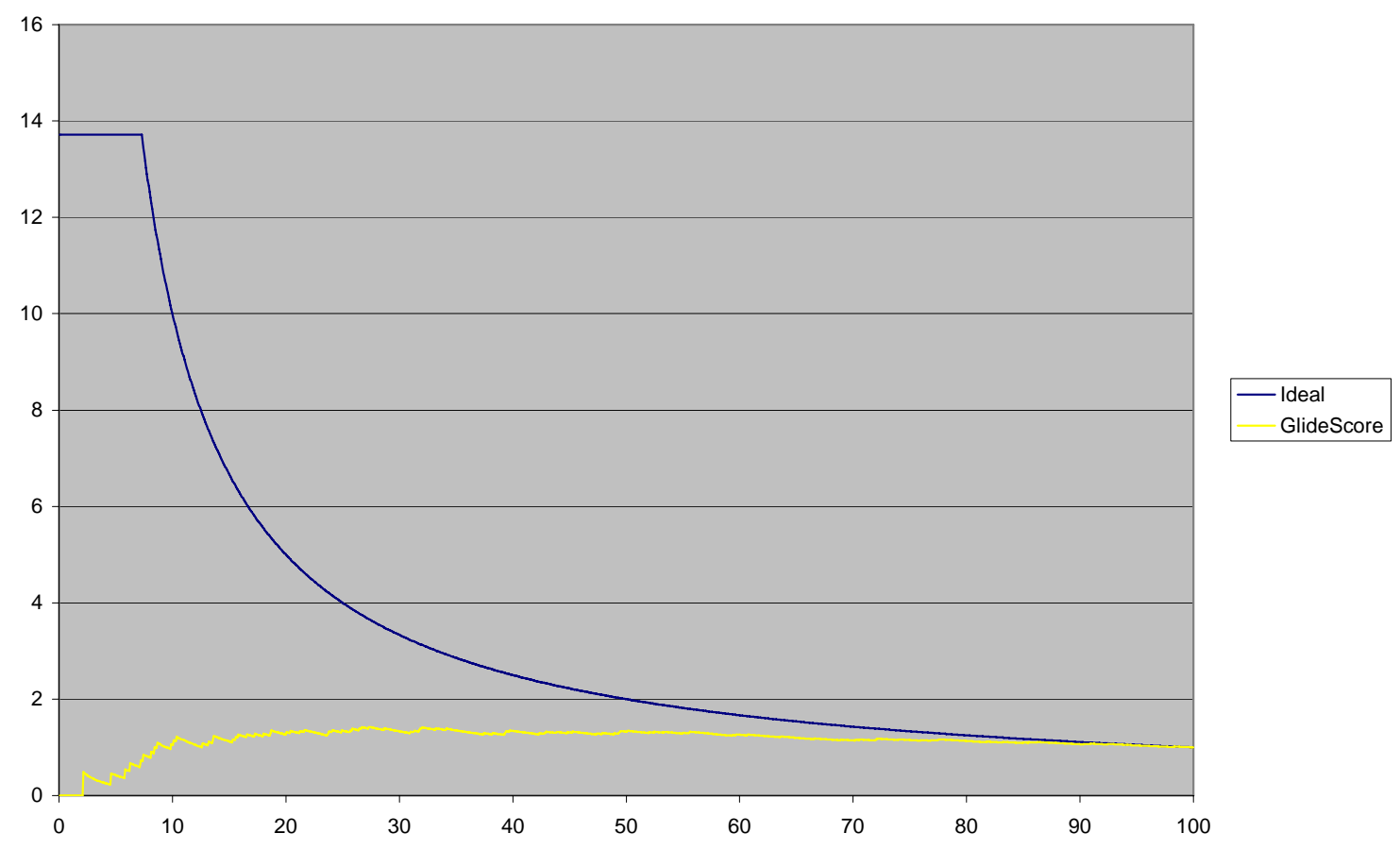


Gold

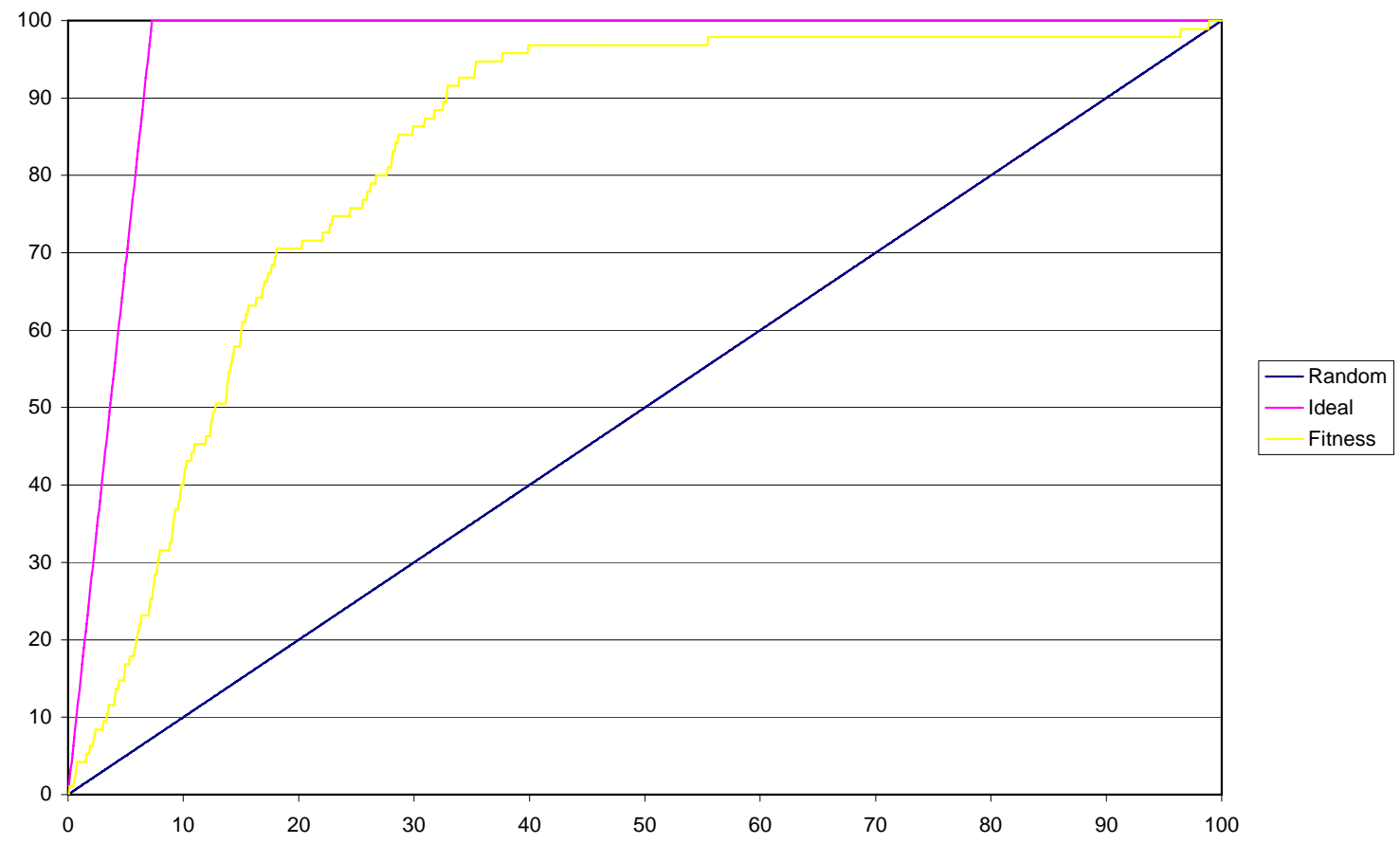

Gold

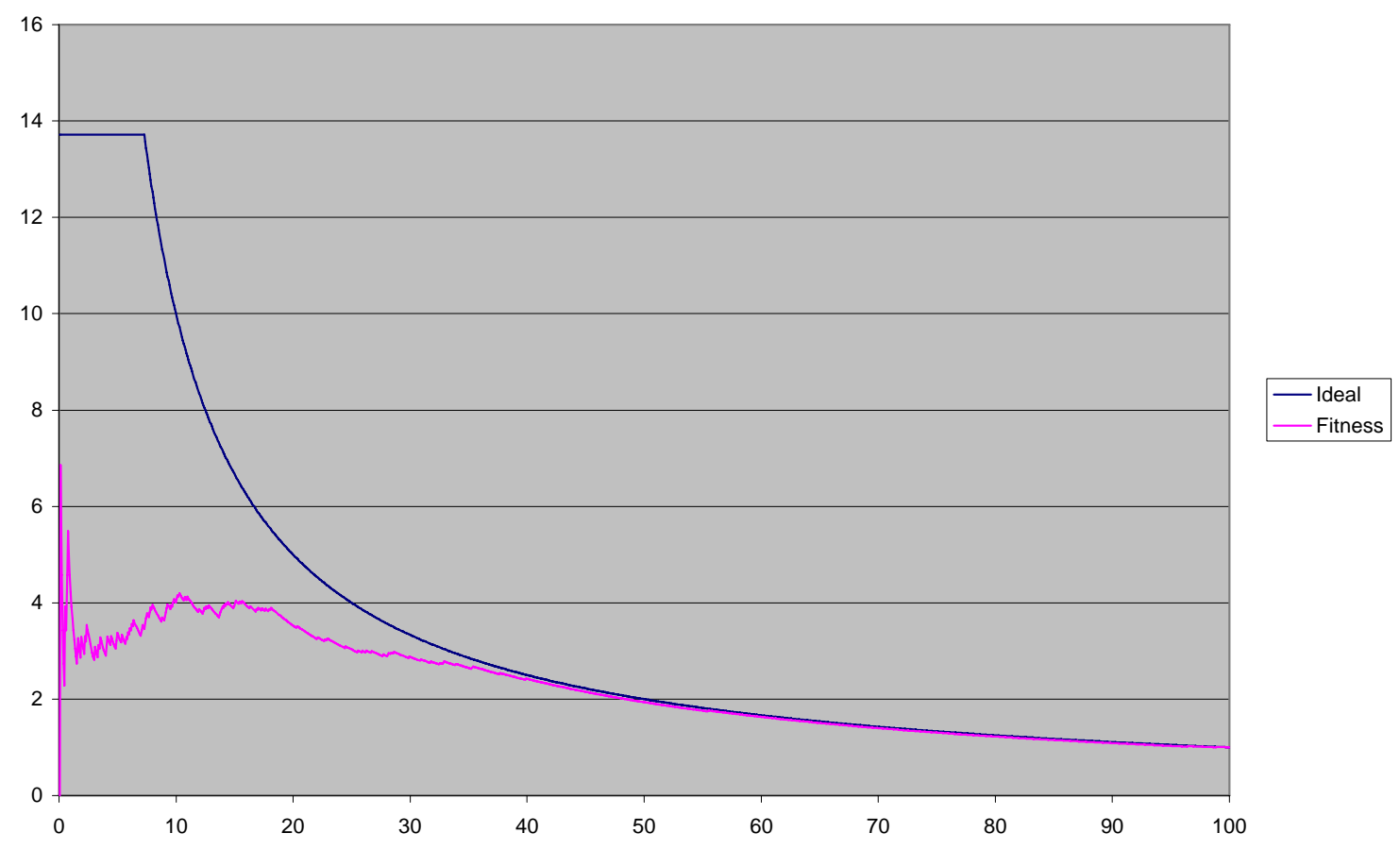




\section{LigFit}

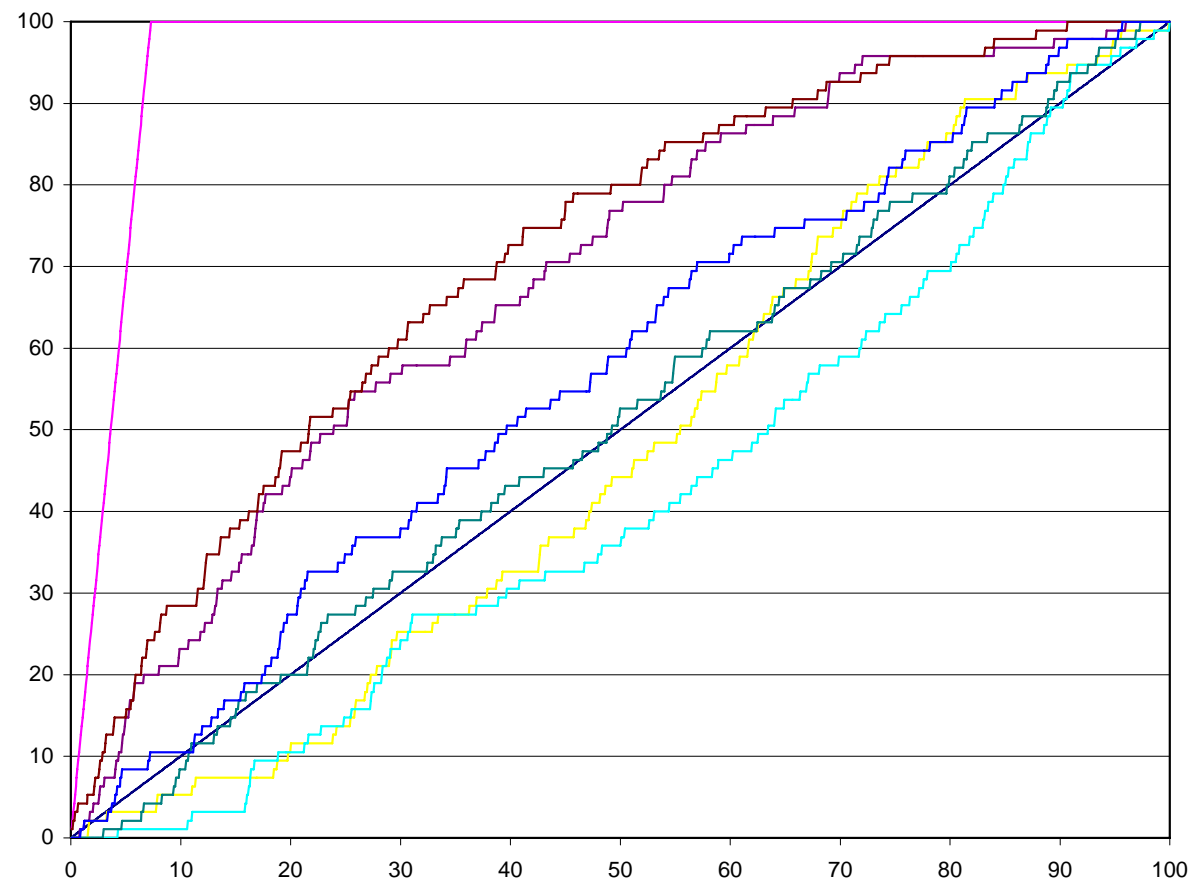

- Random
- Ideal
DrugScore, CFF
DrugScore, Dreiding
- LigScore2, CFF
- LigScore2, Dreiding
PMF, CFF
PMF, Dreiding

LigFit

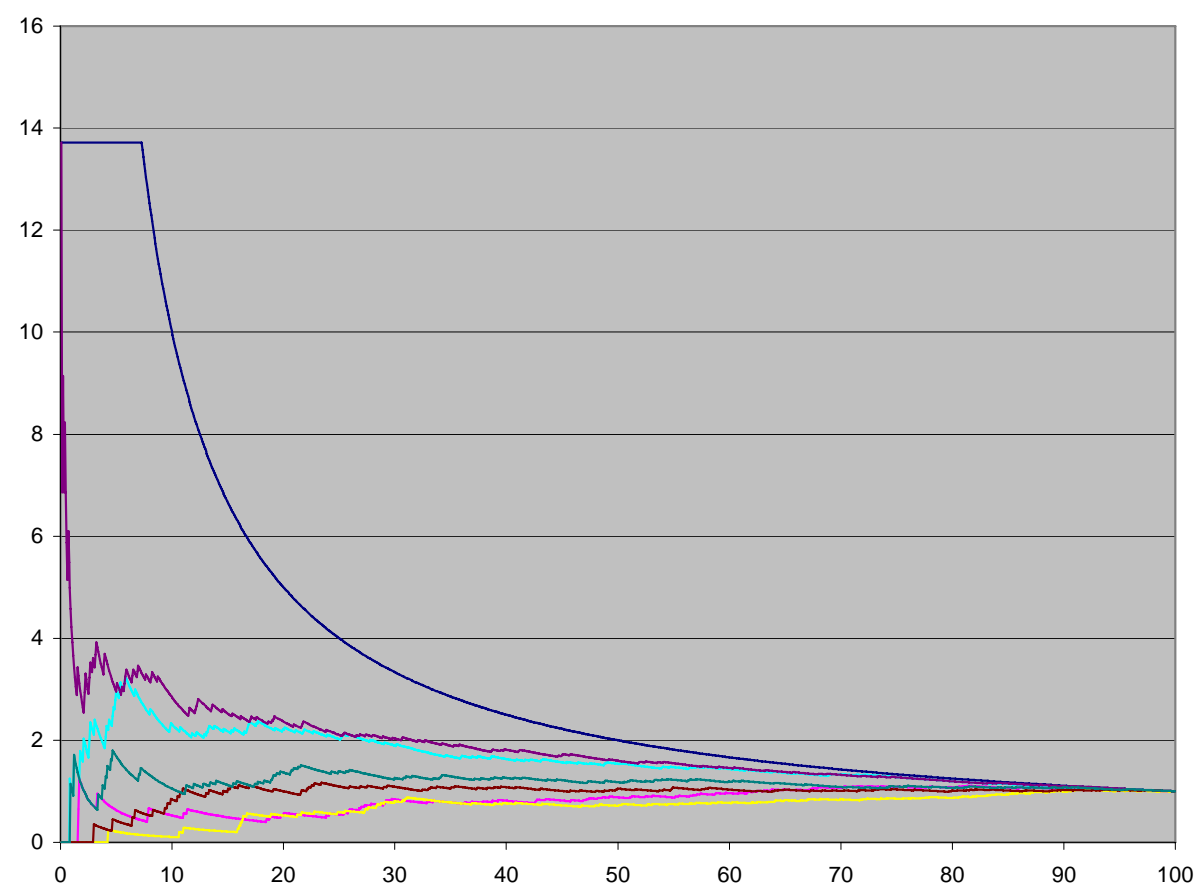


MOE

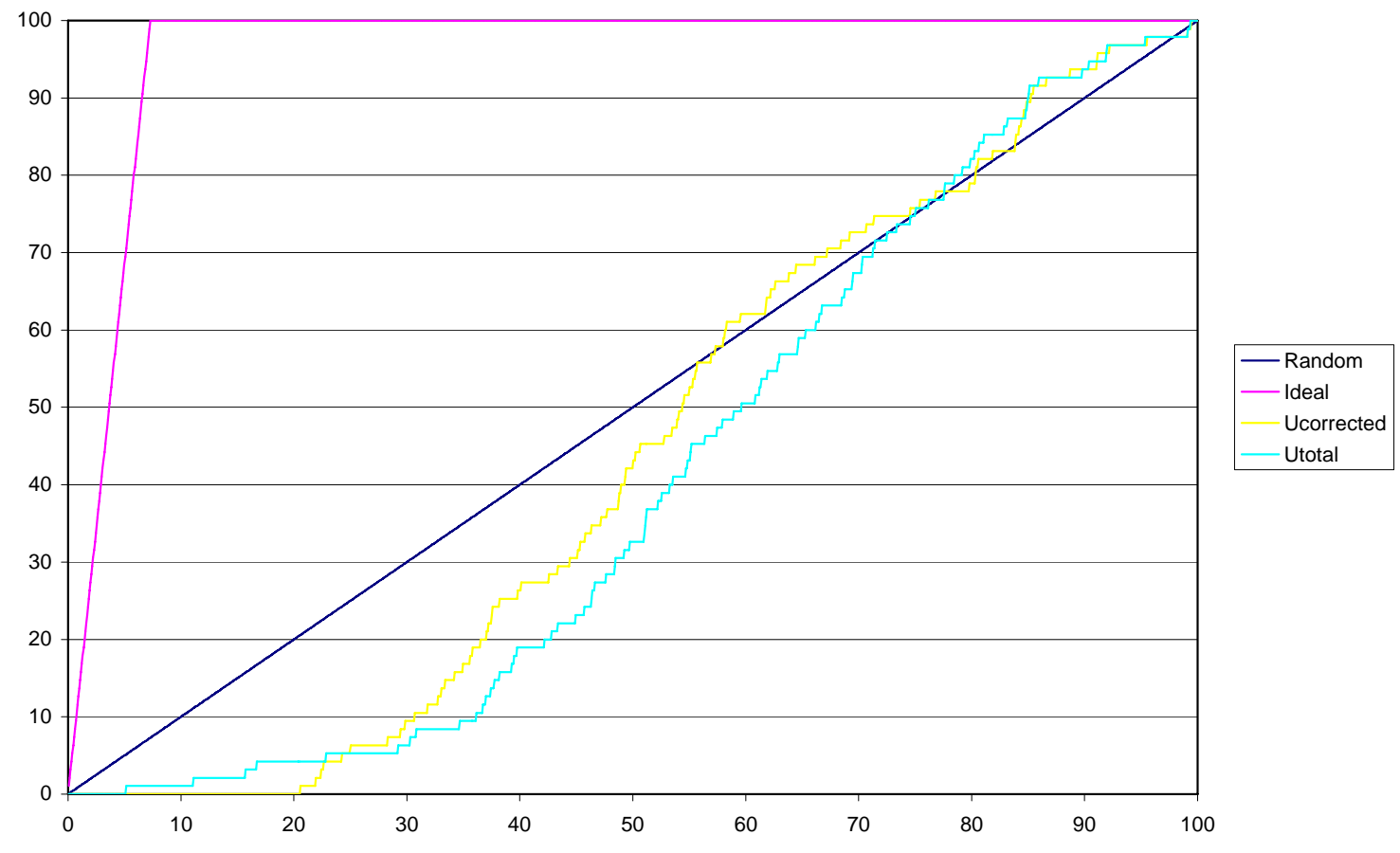

MOE

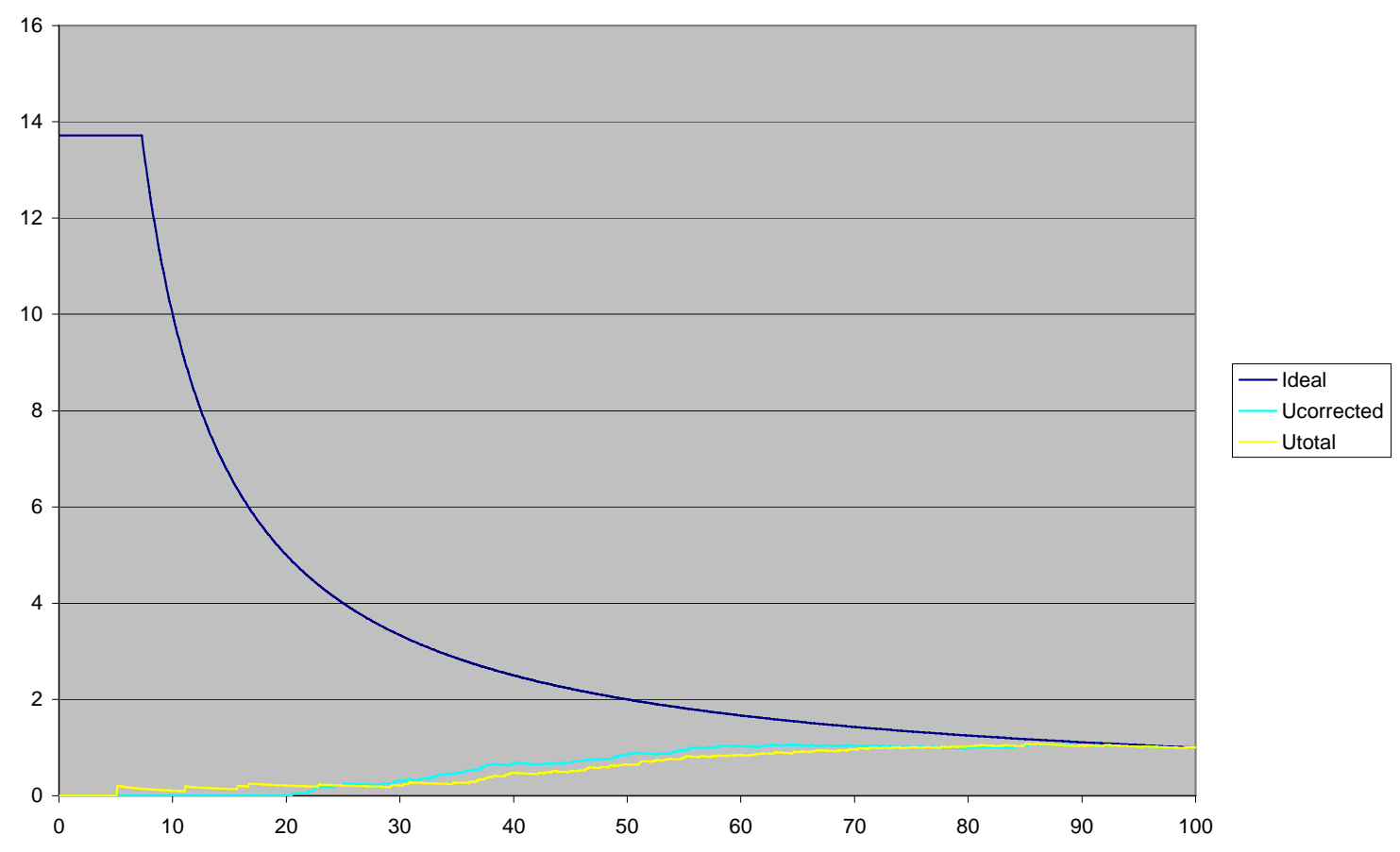


MVP

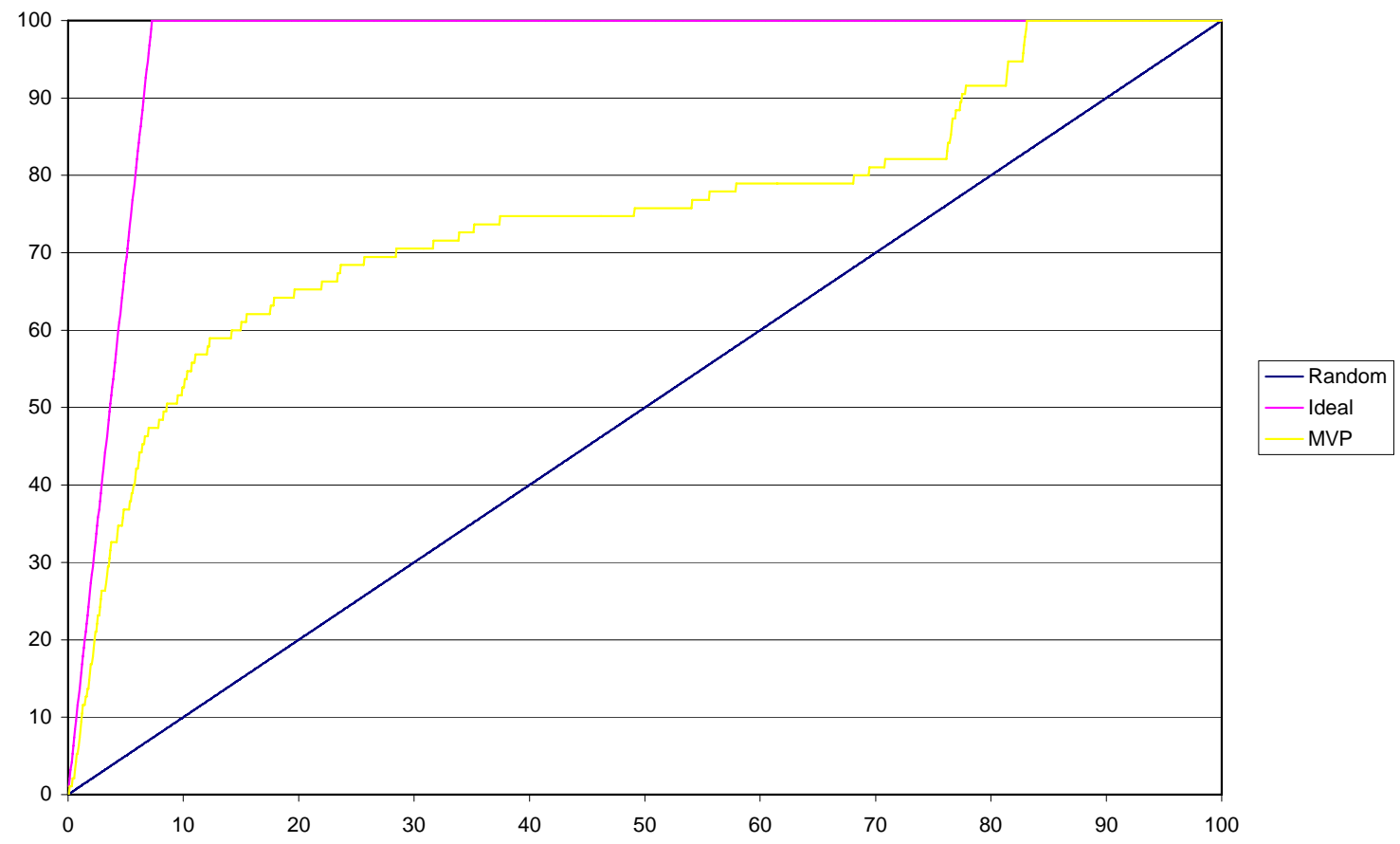

MVP

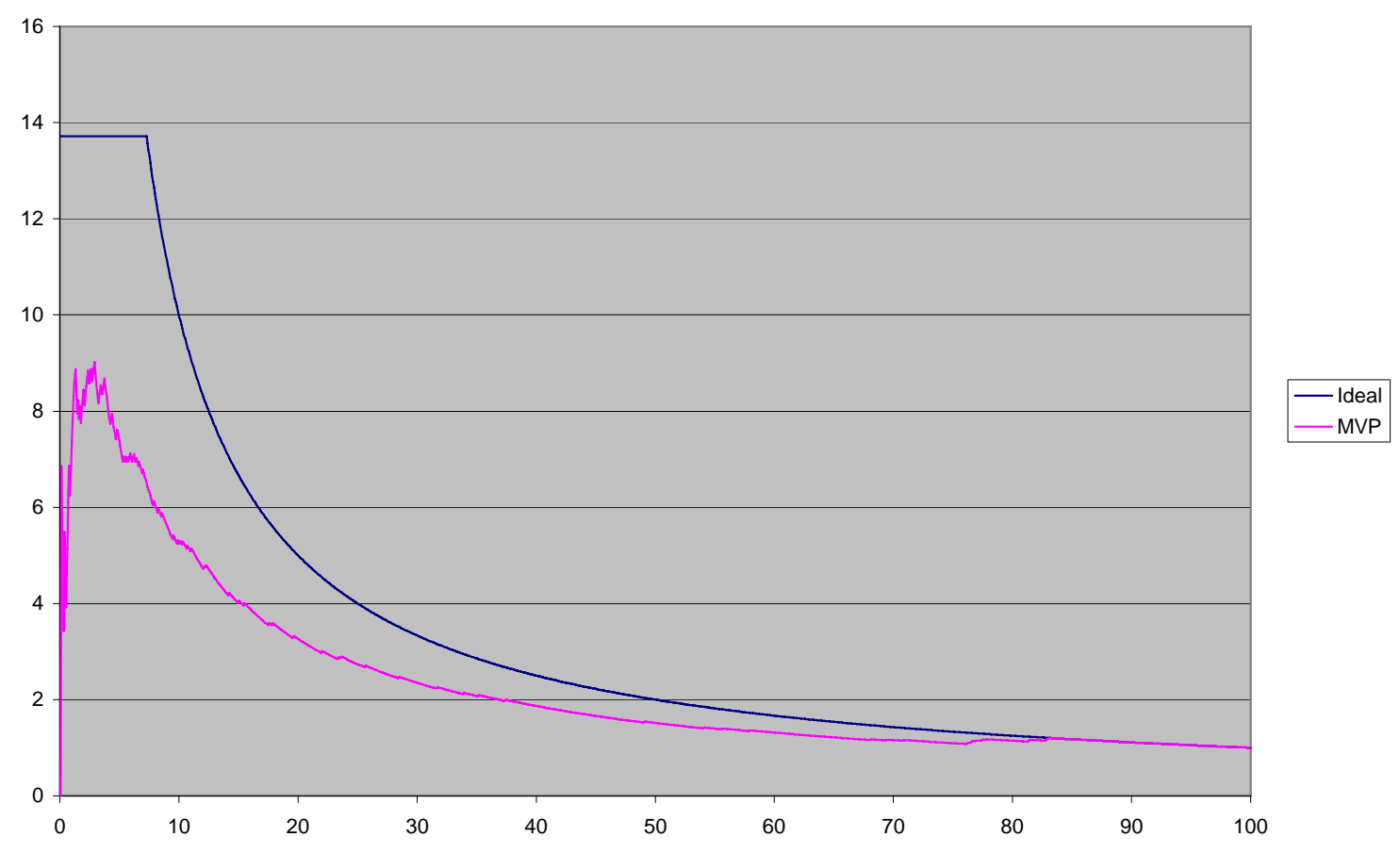




\section{Dock4}

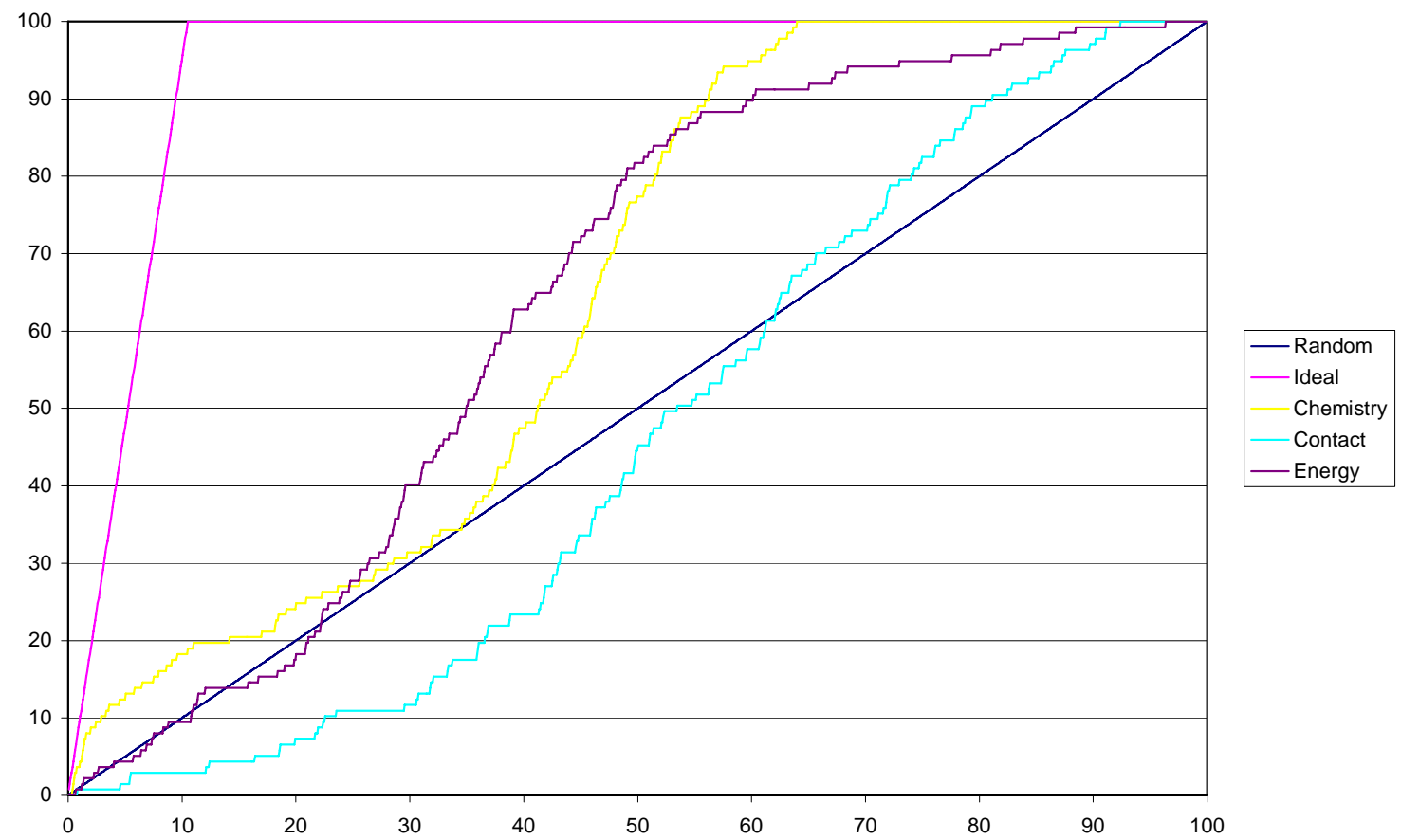

Dock4

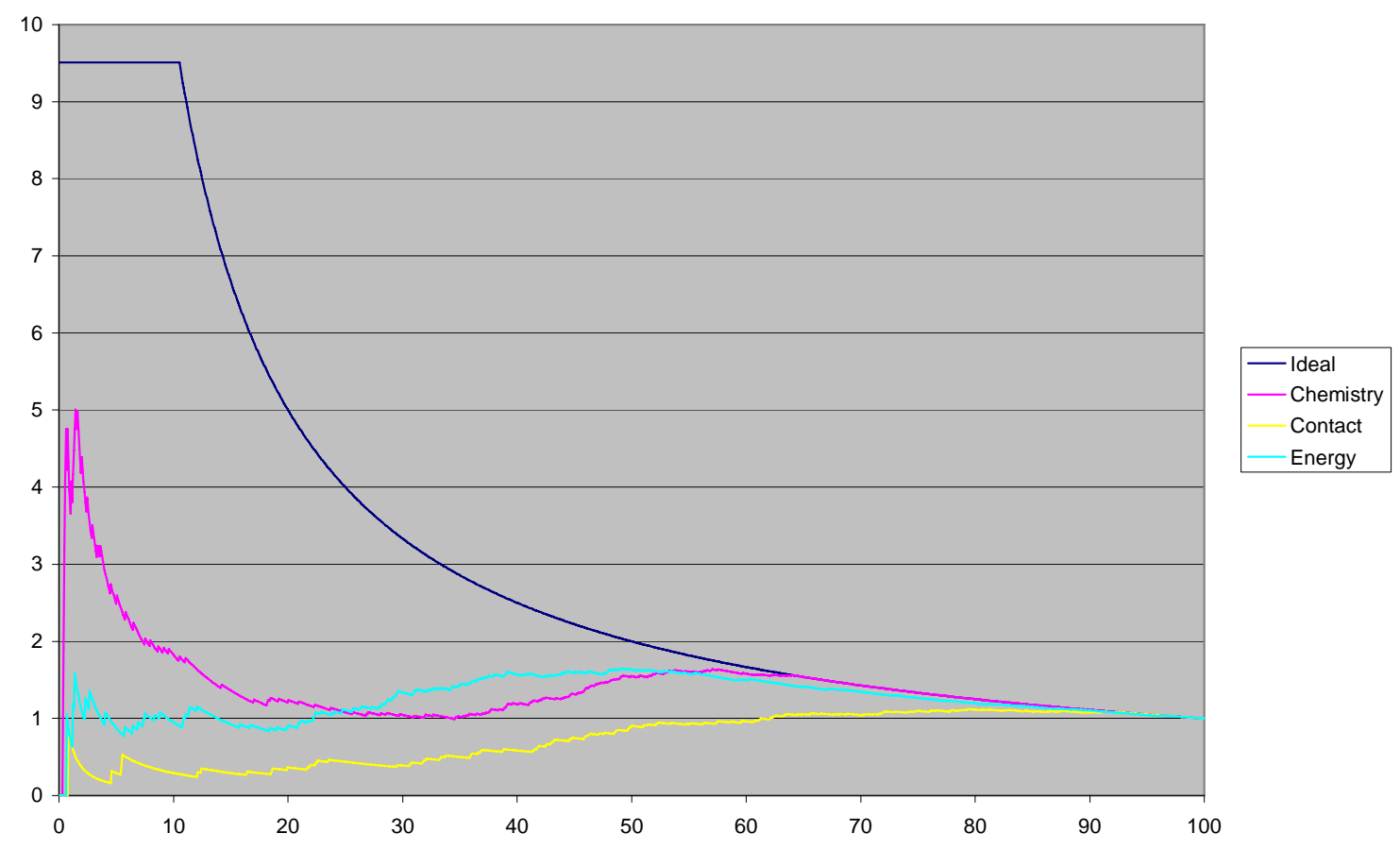




\section{Dockit}

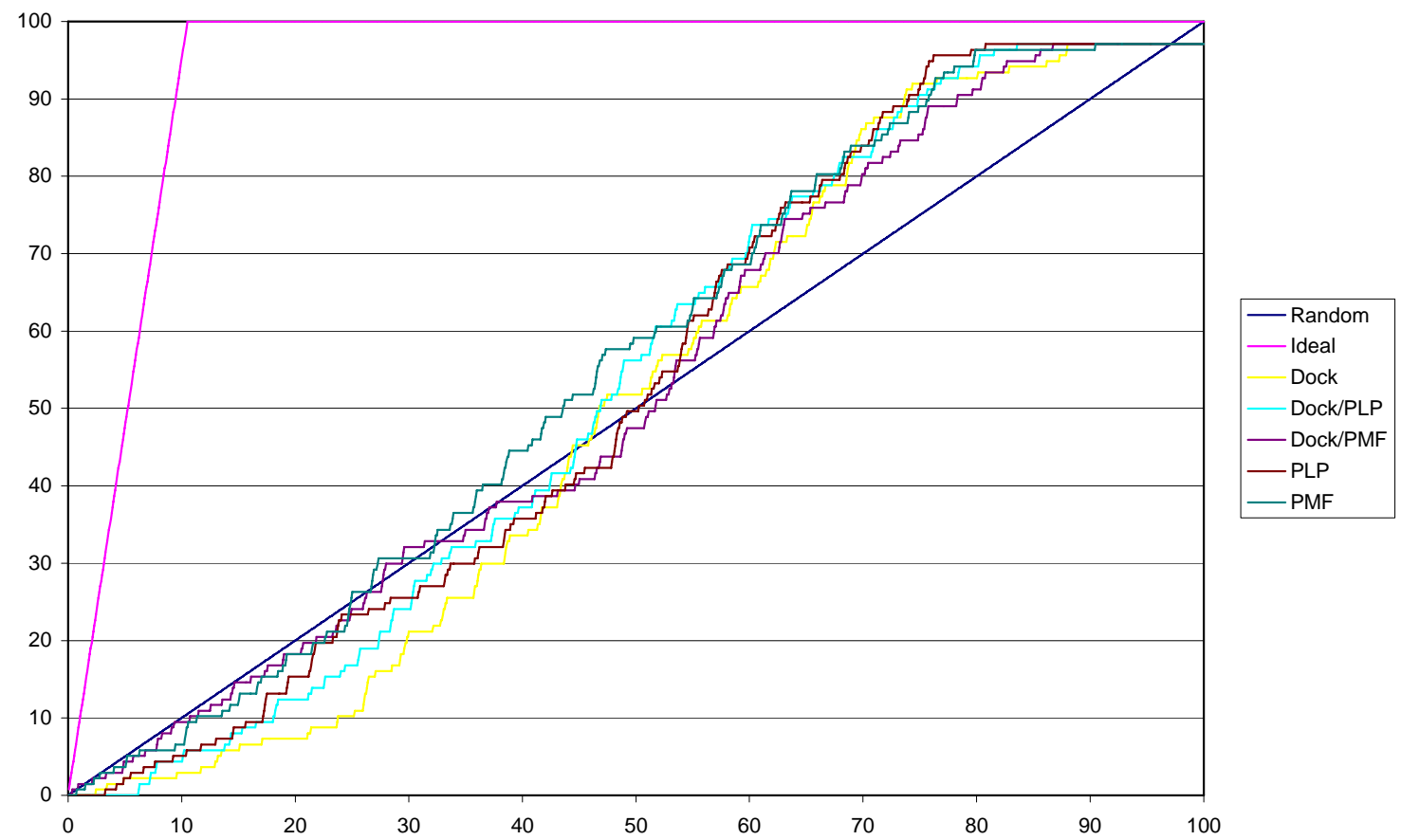

Dockit

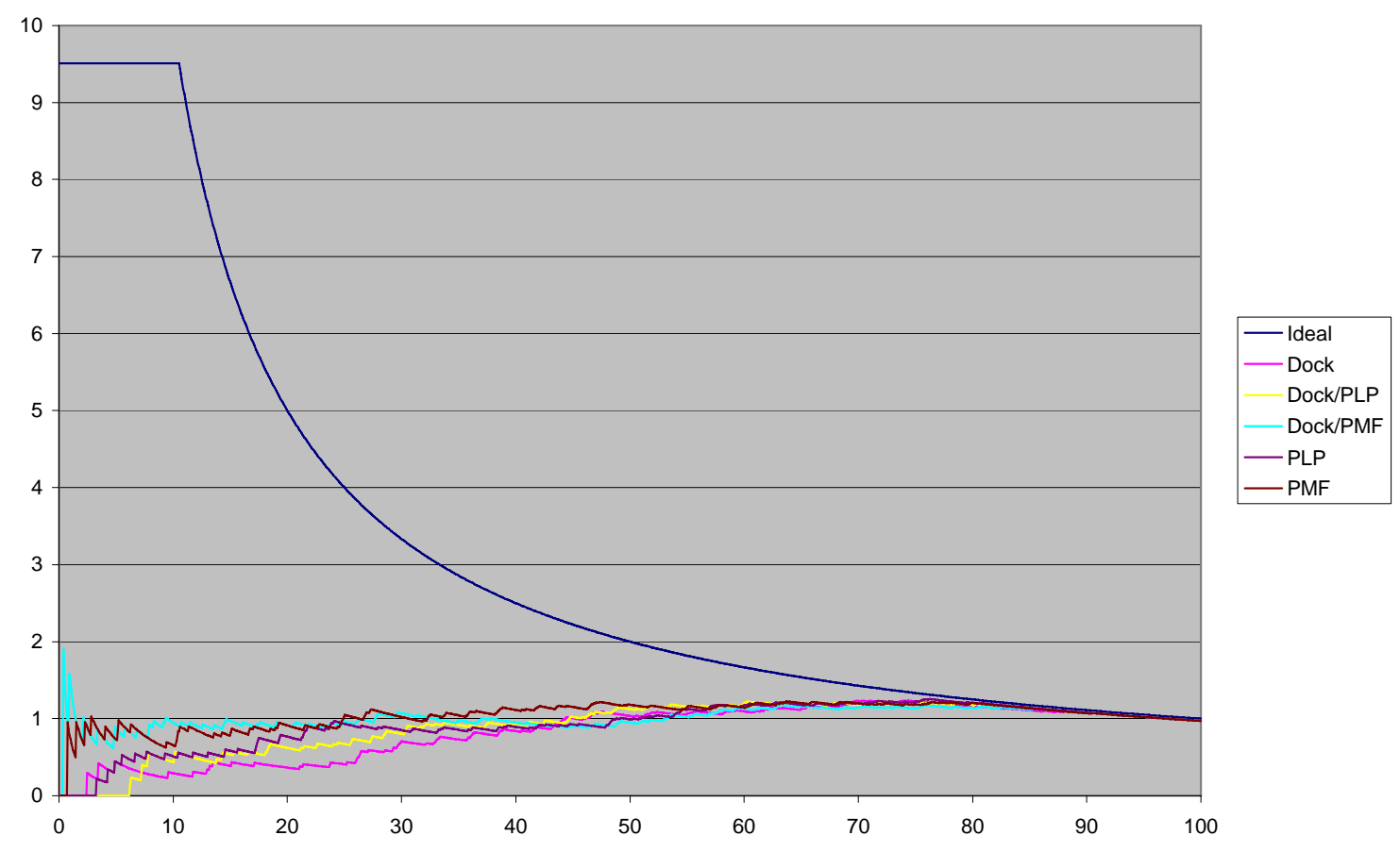


FlexX

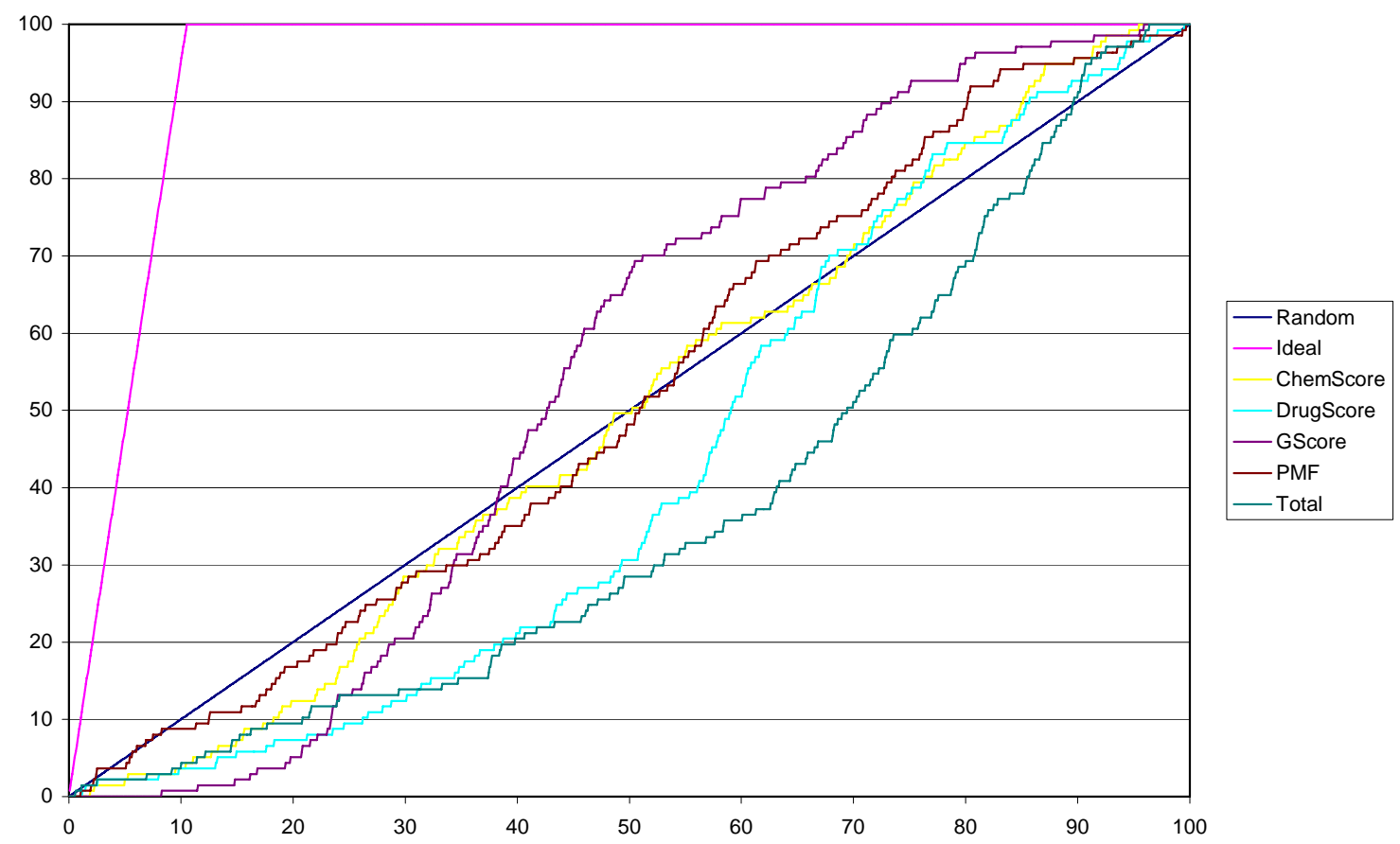

FlexX

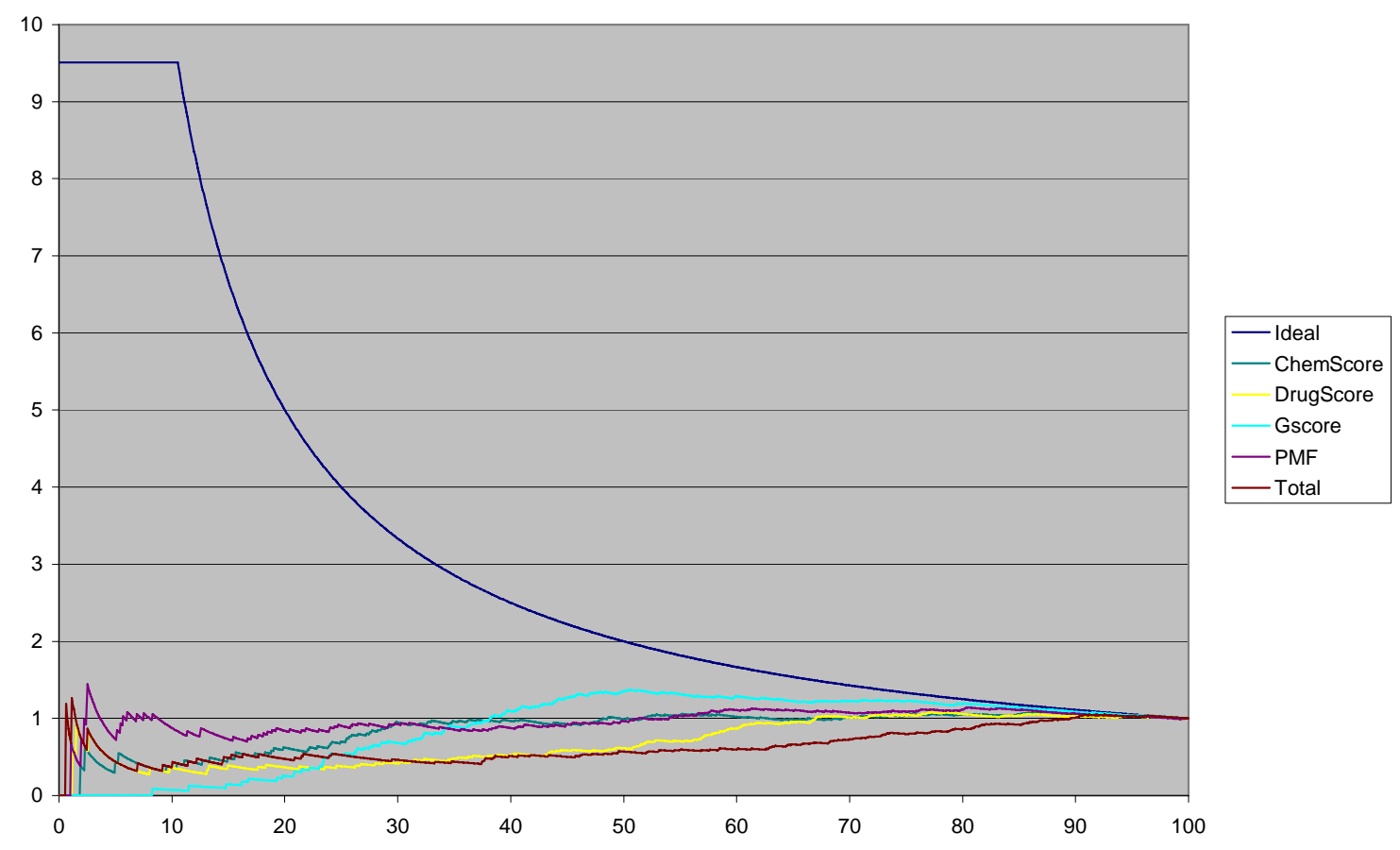


Flo

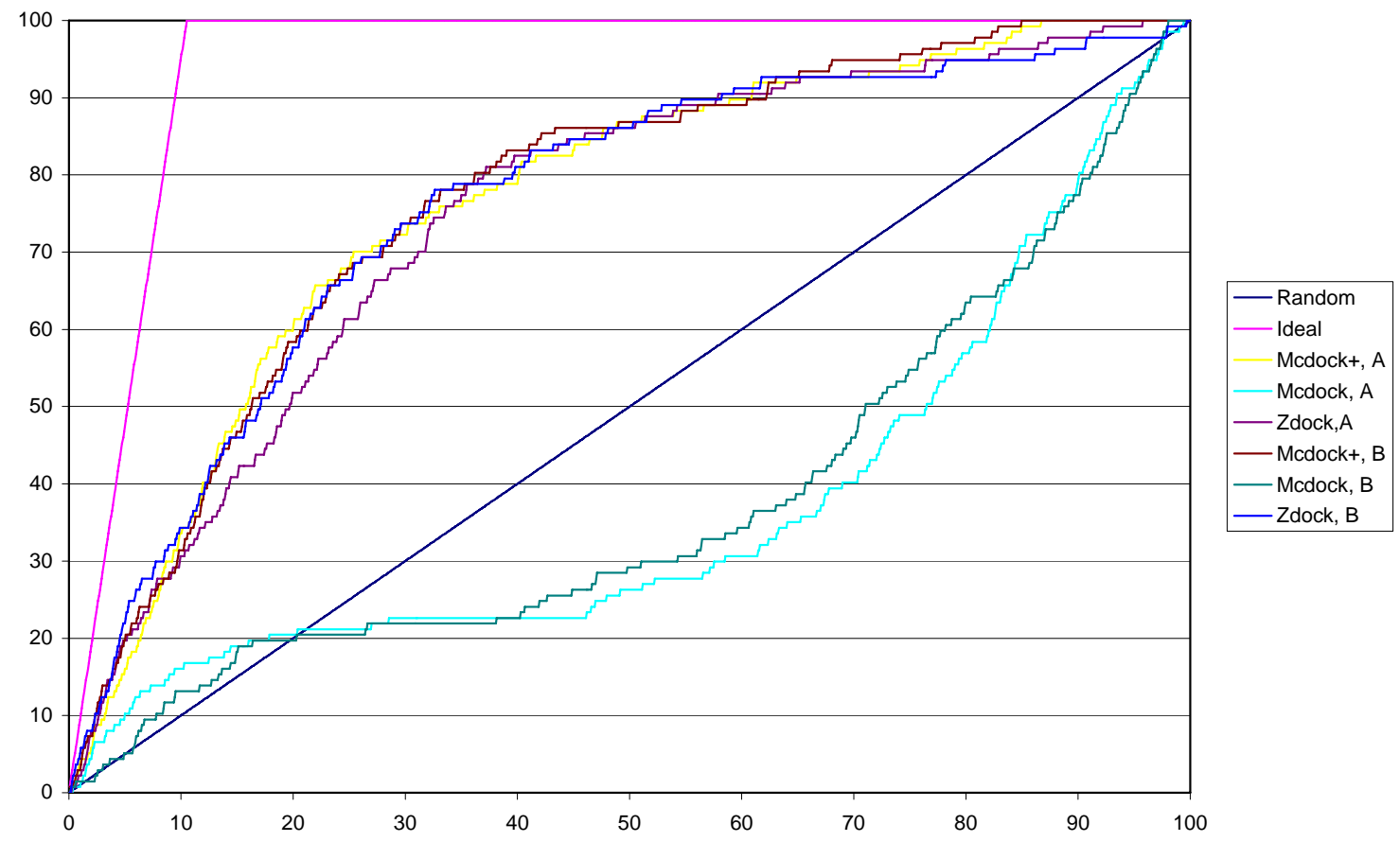

Flo

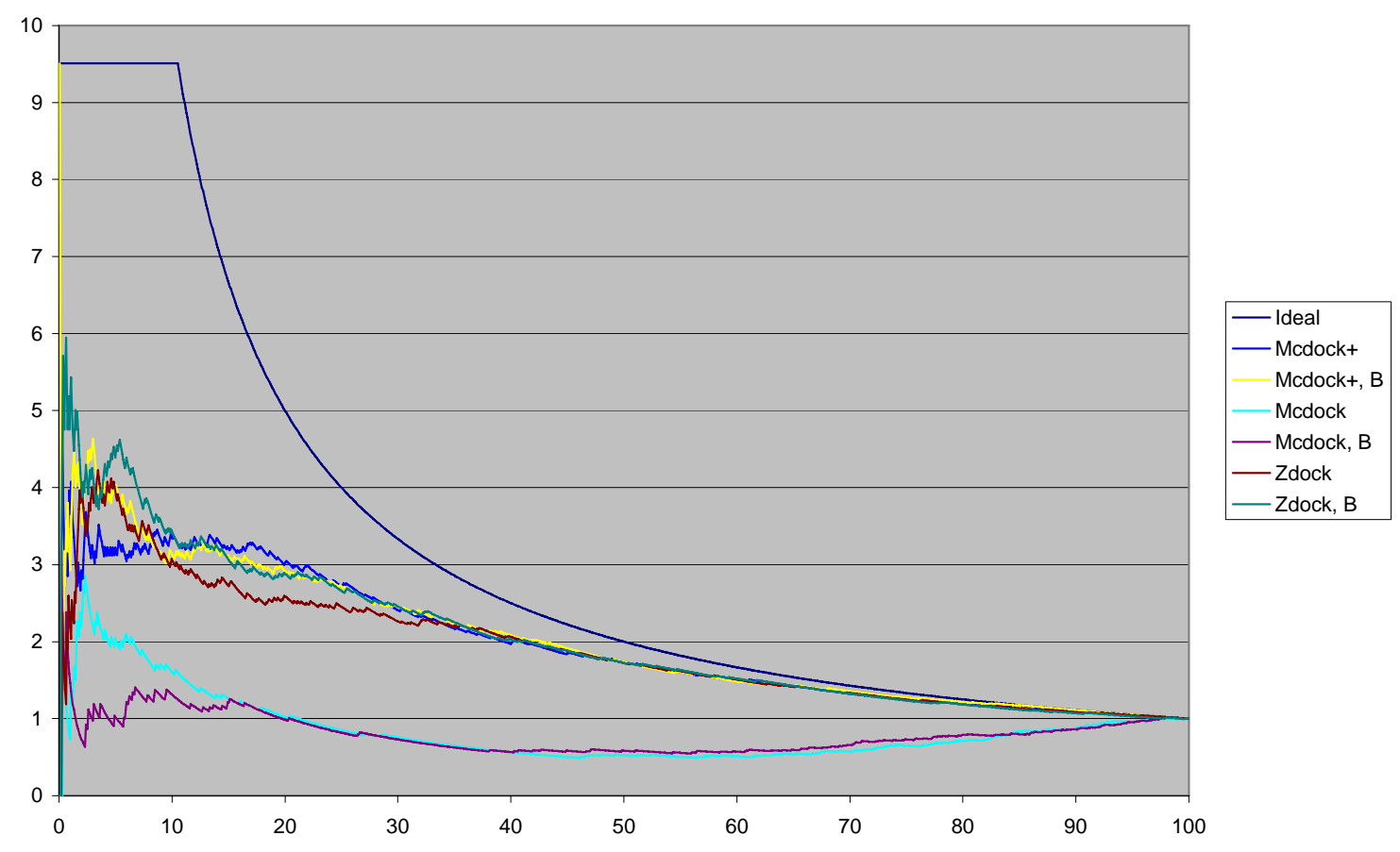


Fred

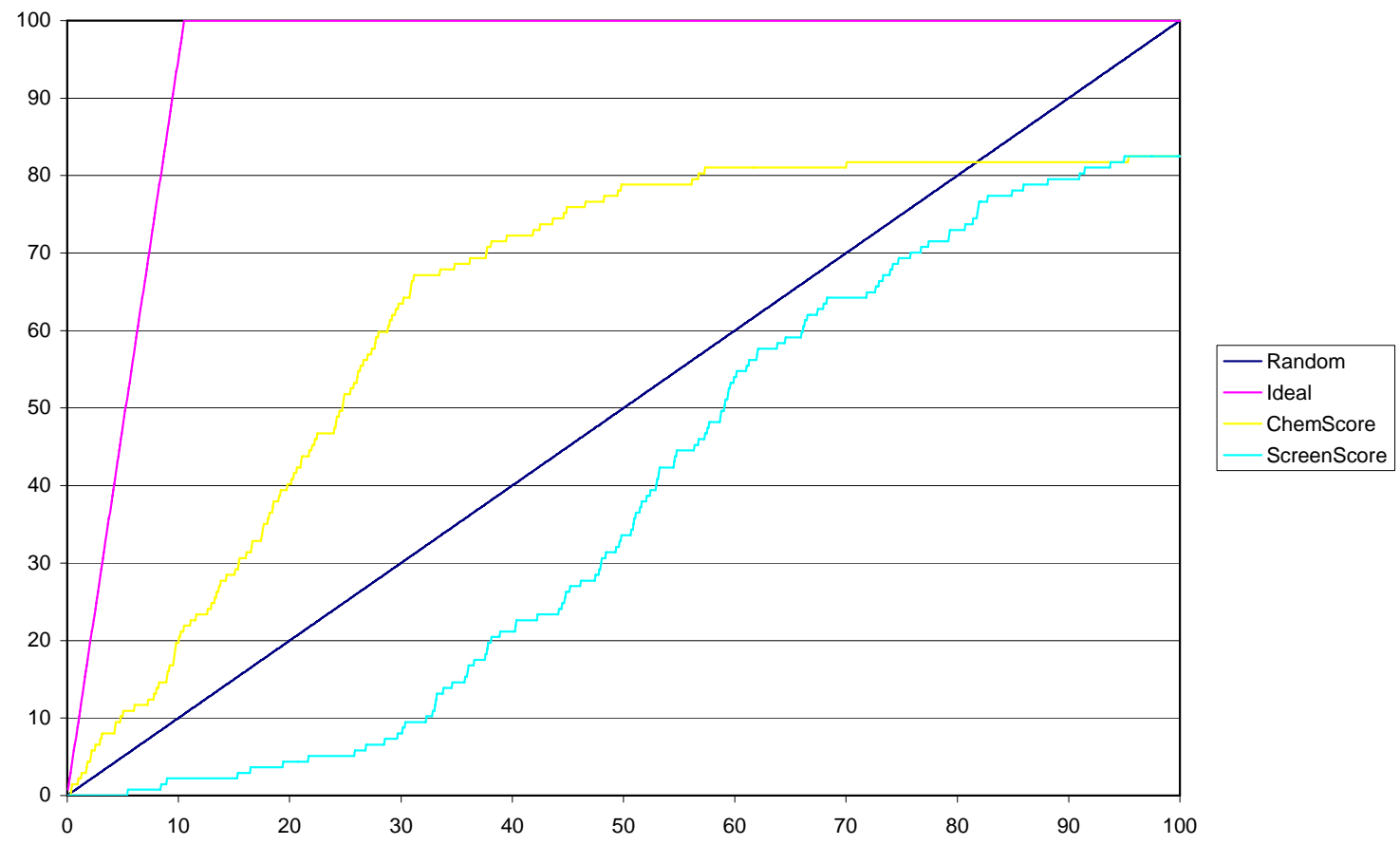

Fred

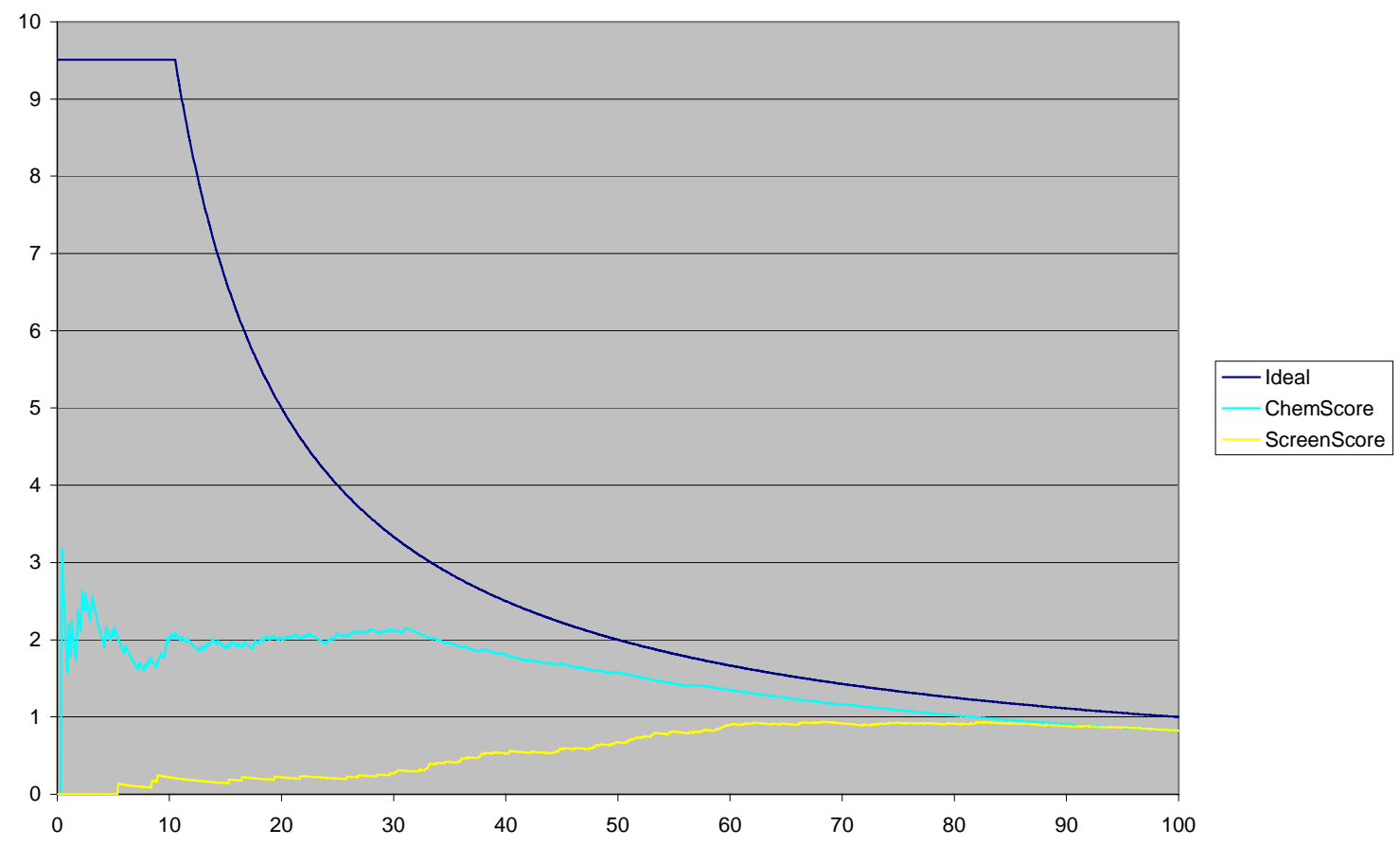




\section{Glide}

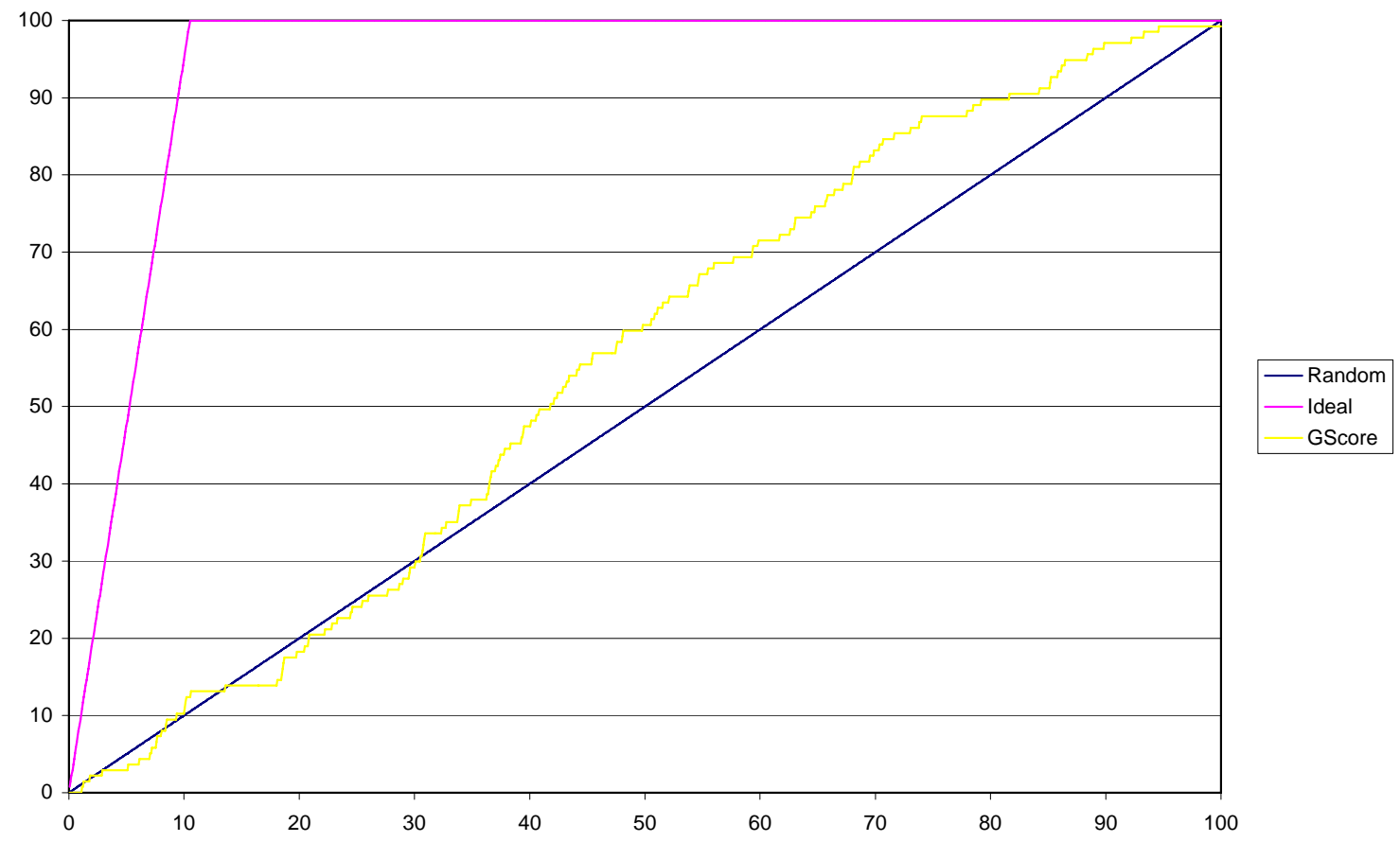

Glide

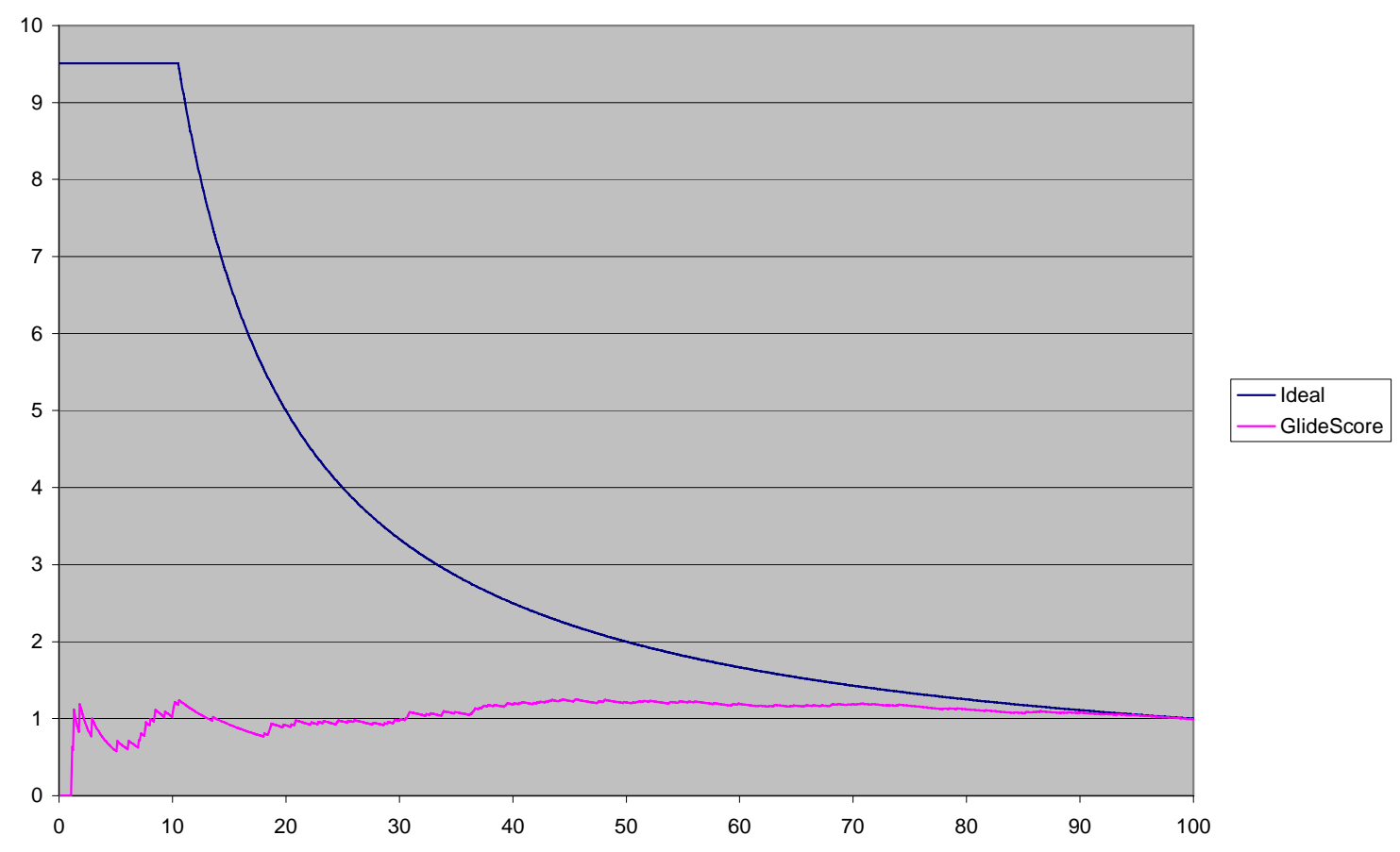


Gold

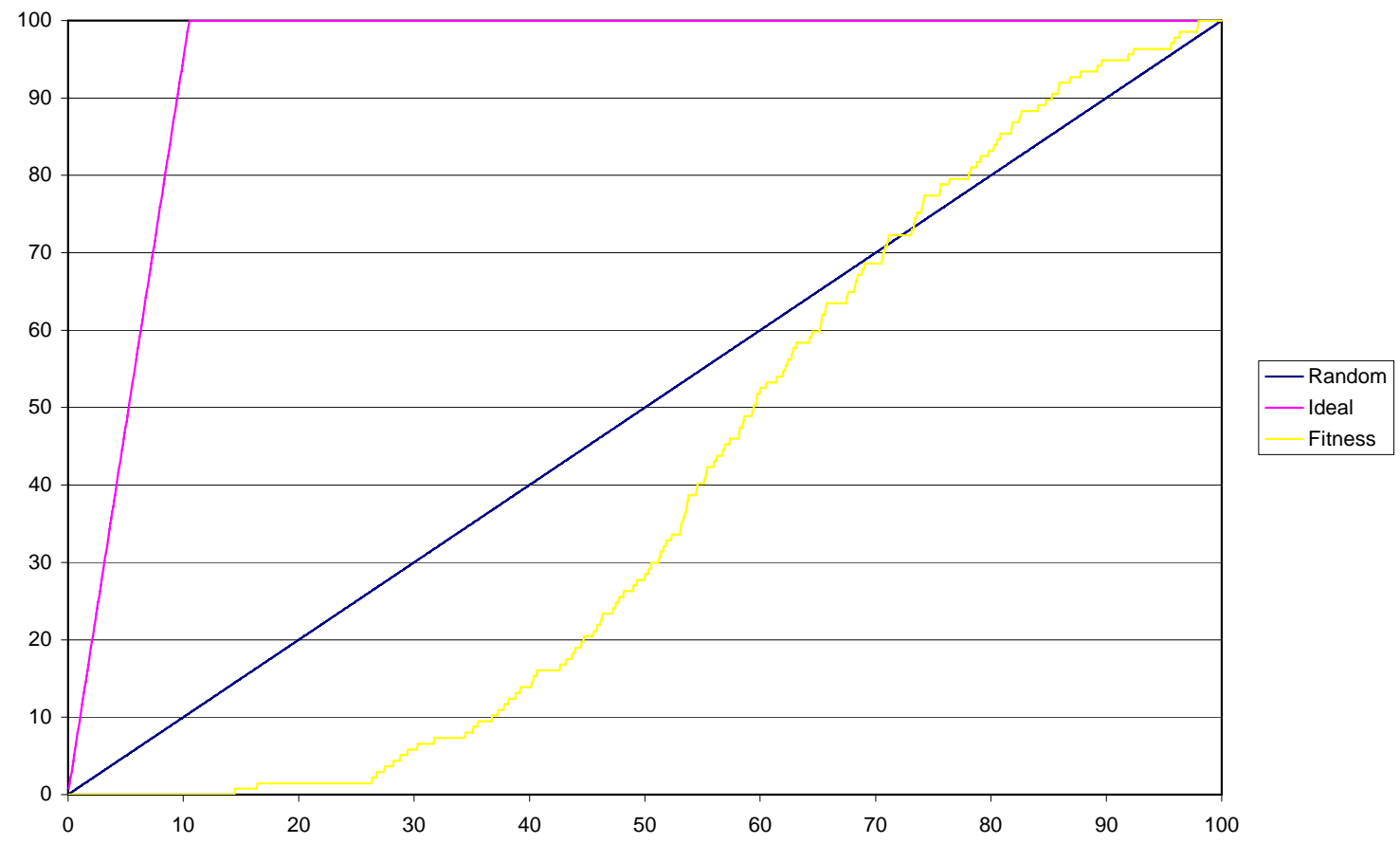

Gold

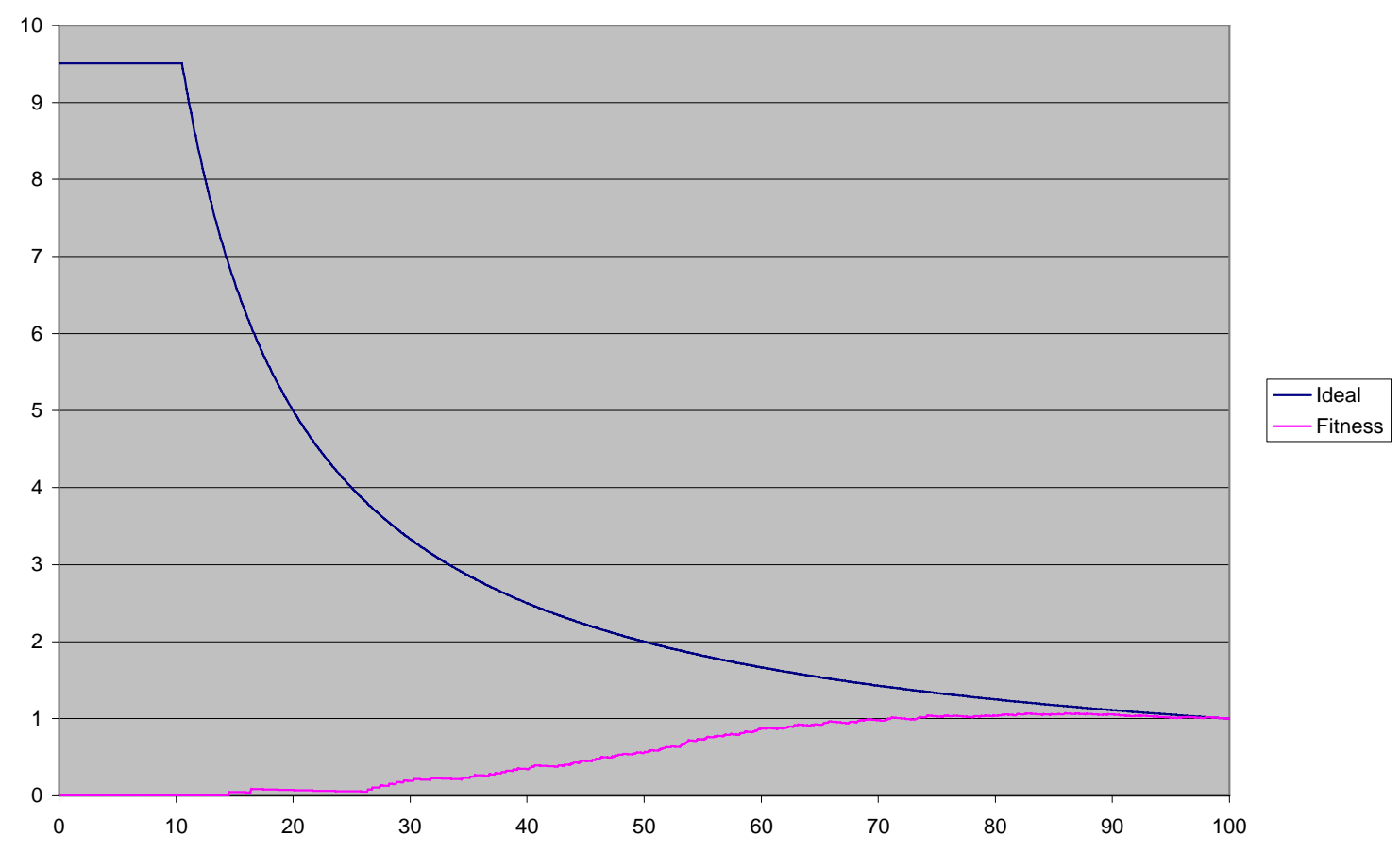


LigFit

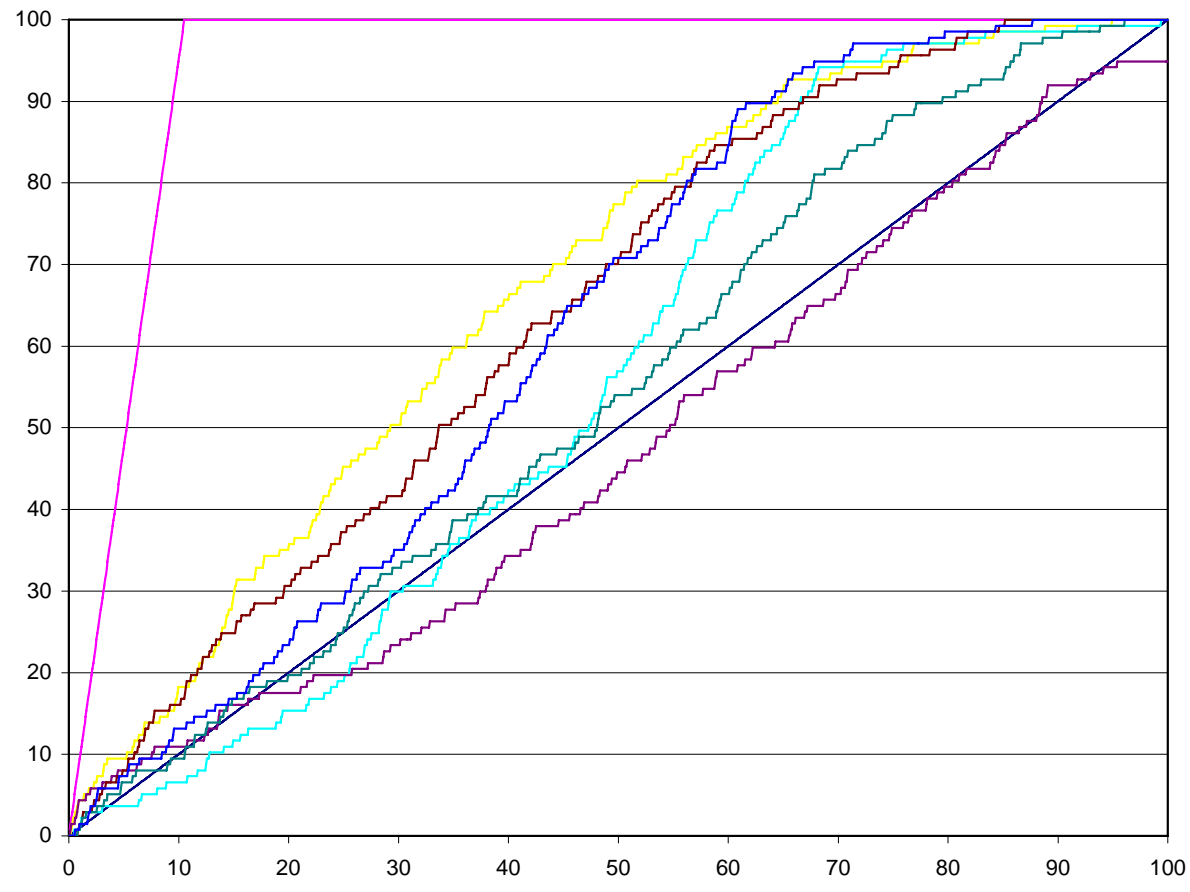

\begin{tabular}{|l|}
\hline - Random \\
— ideal \\
DrugScore, CFF \\
- DrugScore, Dreiding \\
_ Ligscore2, CFF \\
_ Ligscore2, Dreiding \\
- PMF, CFF \\
- PMF, Dreiding \\
\hline
\end{tabular}

LigFit

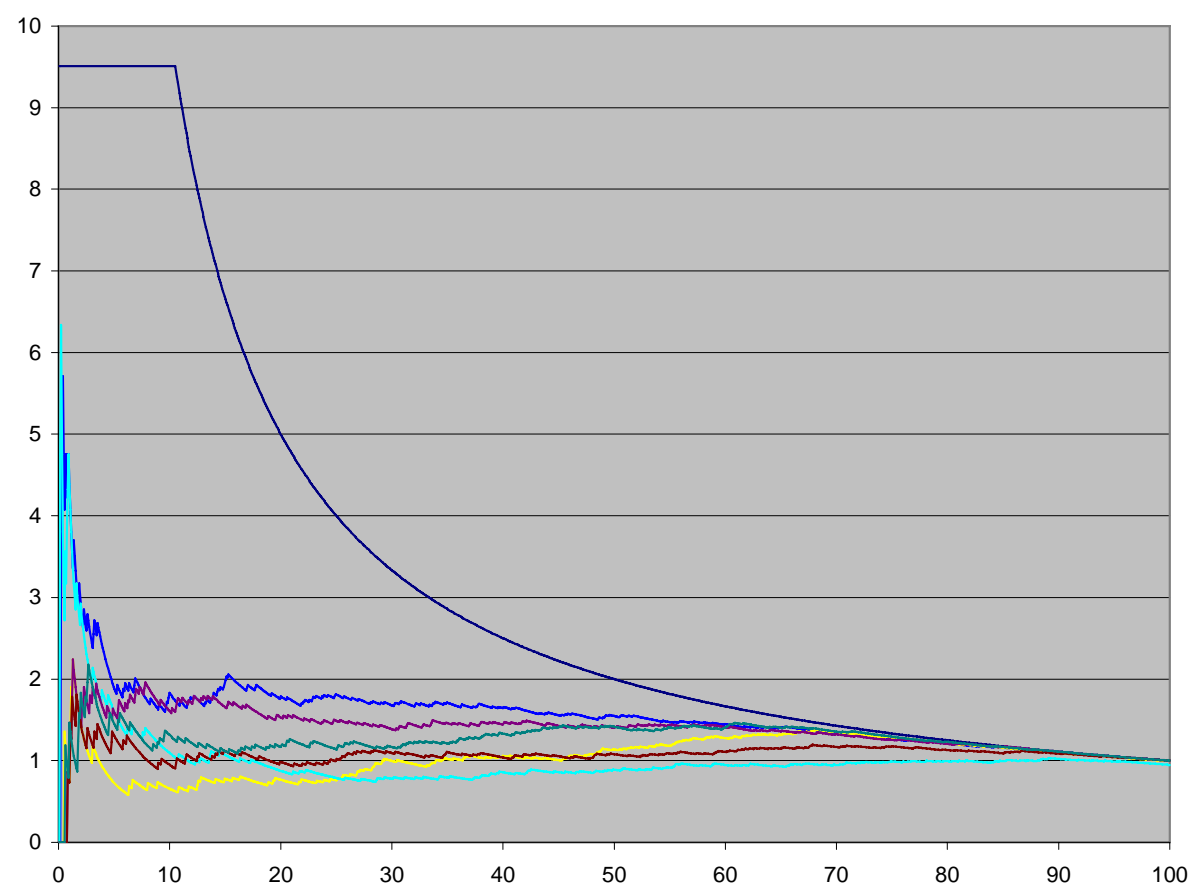

— Ideal
— DrugScore, CFF
DrugScore, Dreiding
— Ligscore2, CFF
- Ligscore2, Dreiding
- PMF, CFF
- PMF, Dreiding


MOE

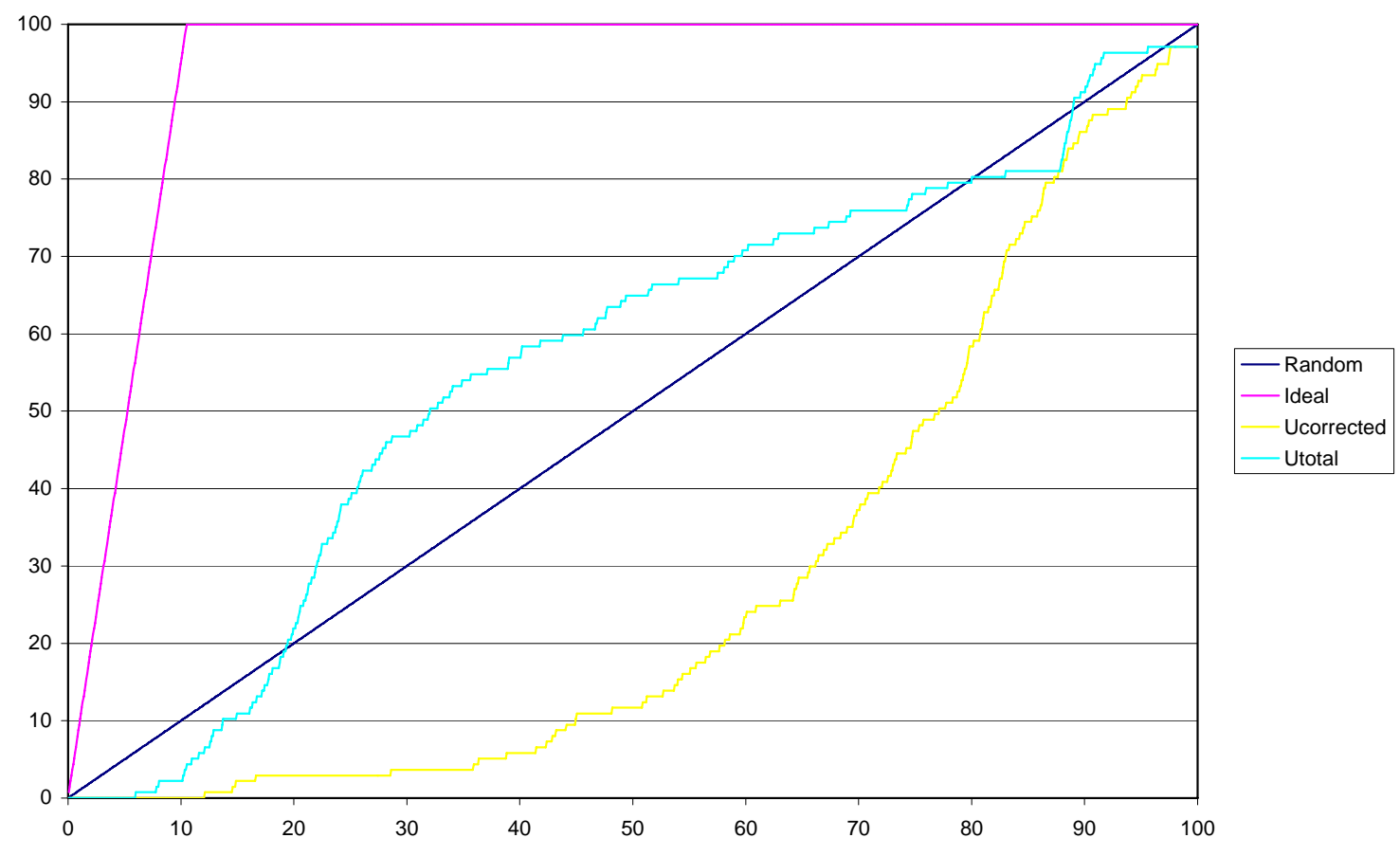

MOE

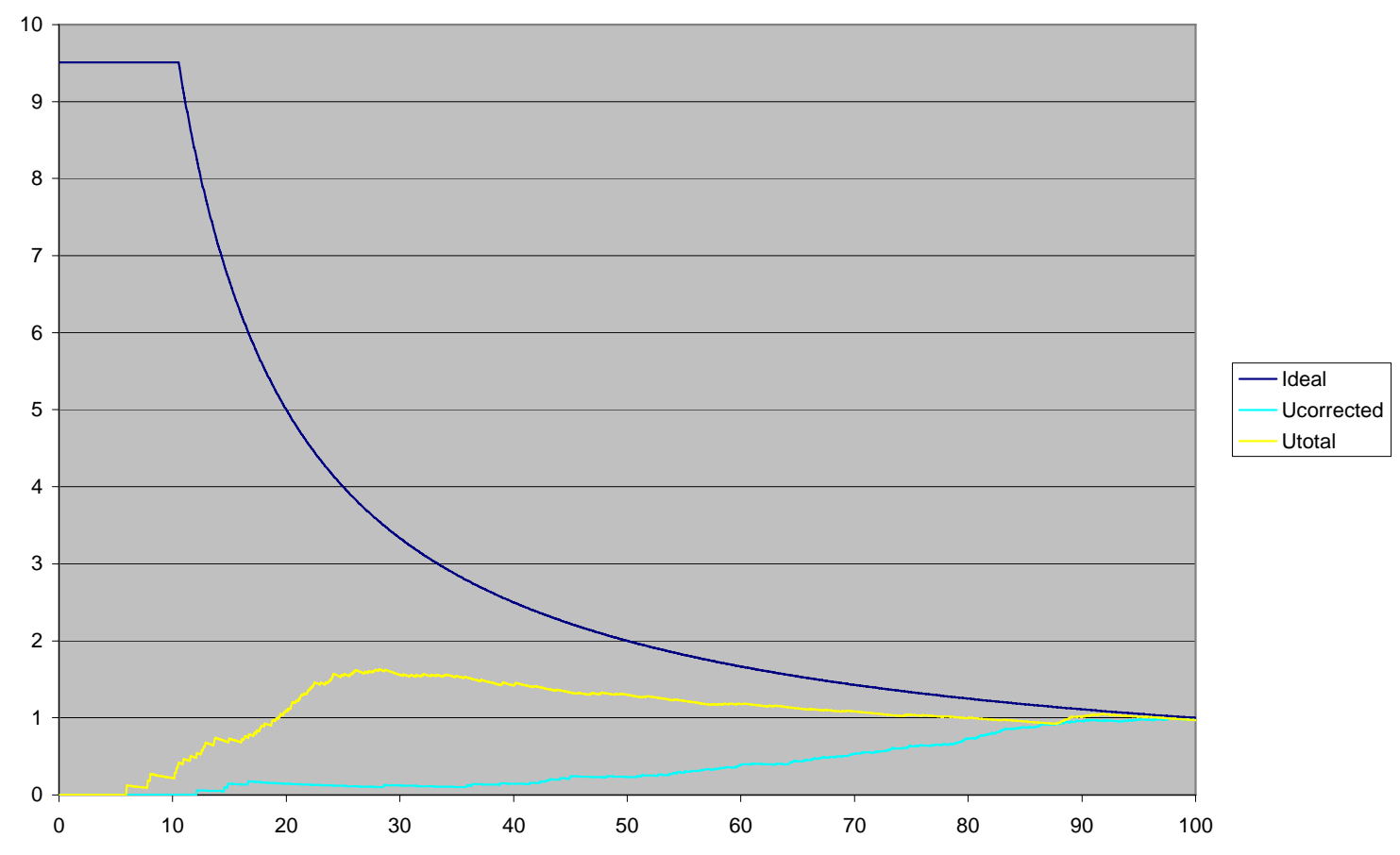


MVP

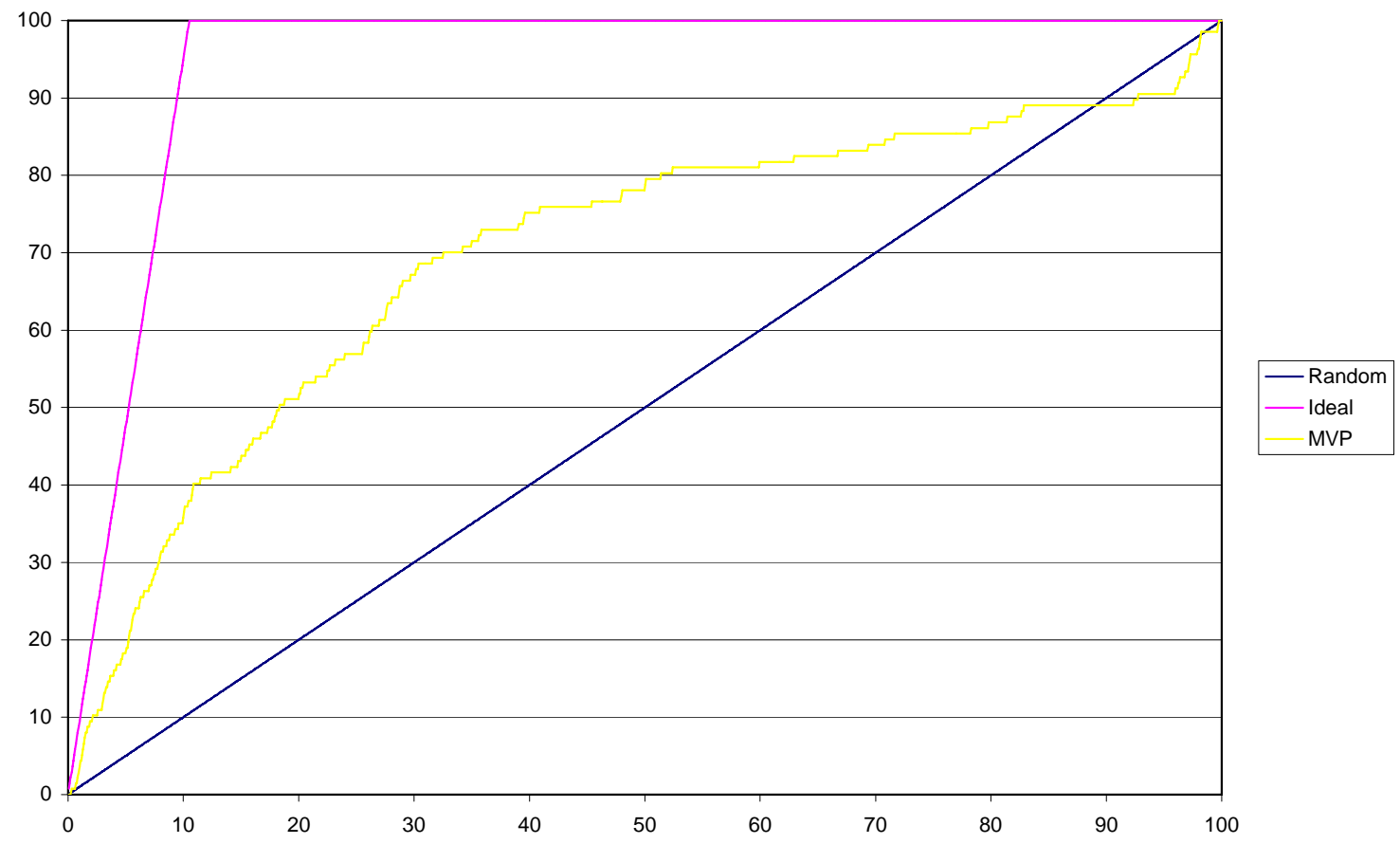

MVP

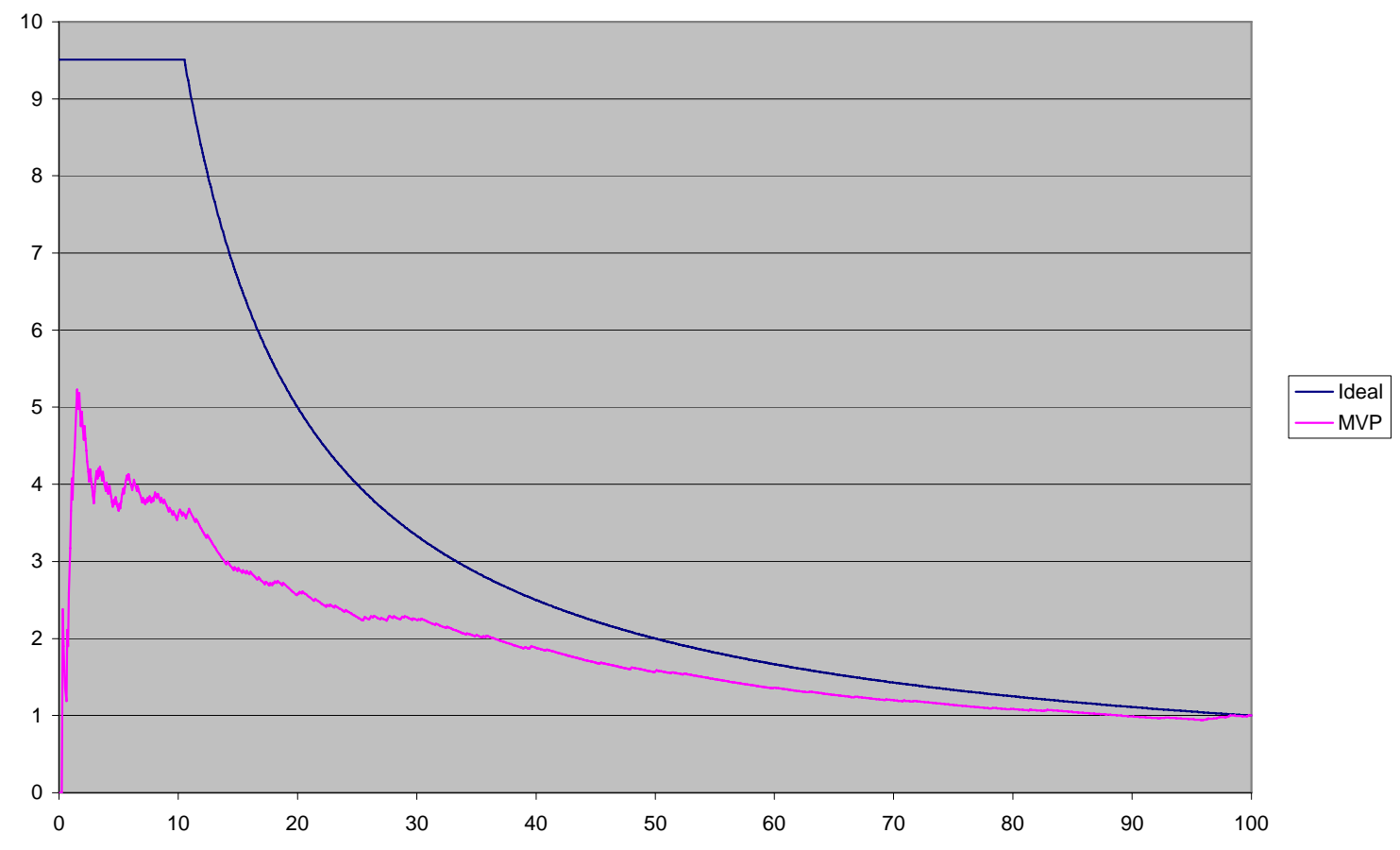




\section{Dock4}

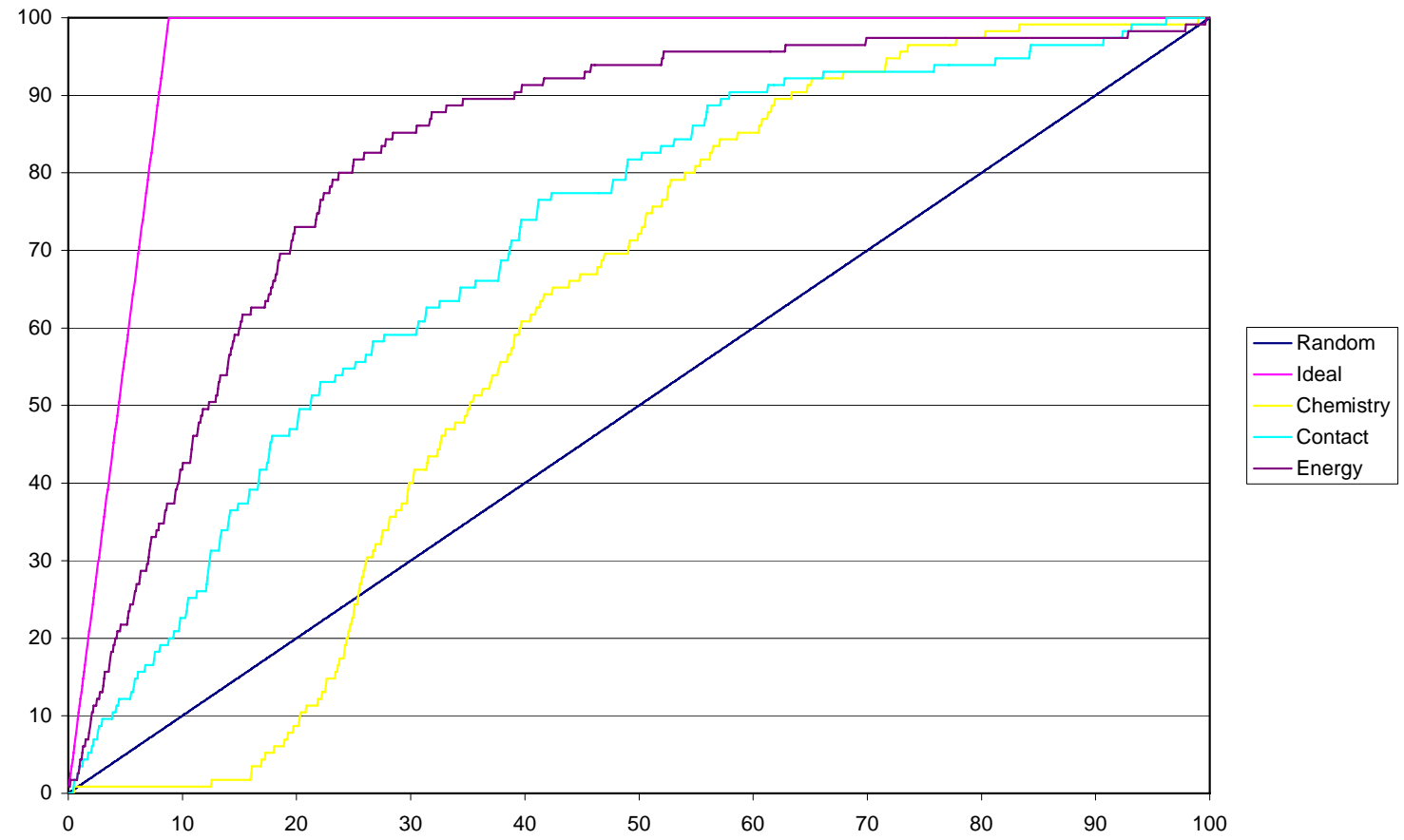

Dock

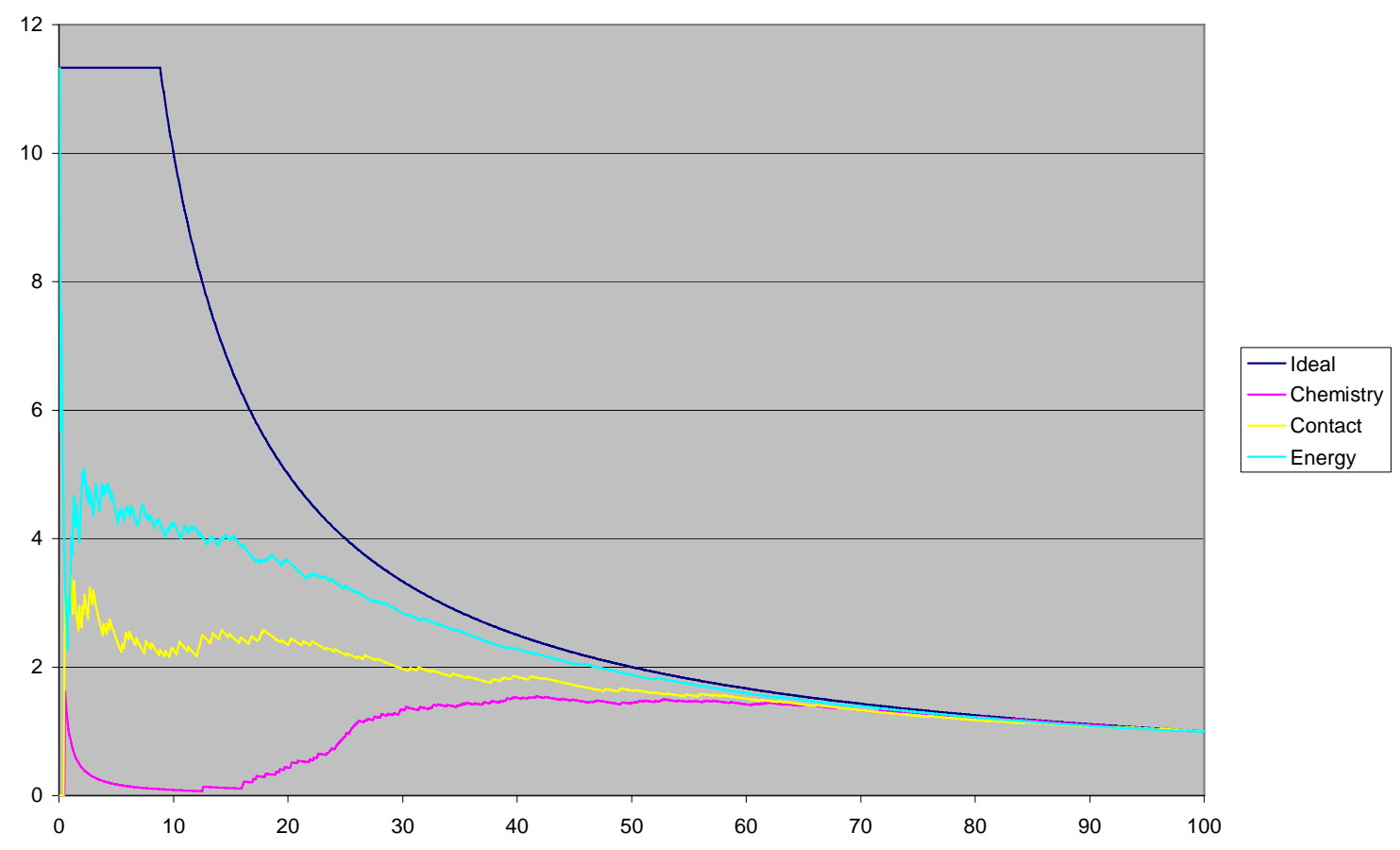




\section{Dockit}

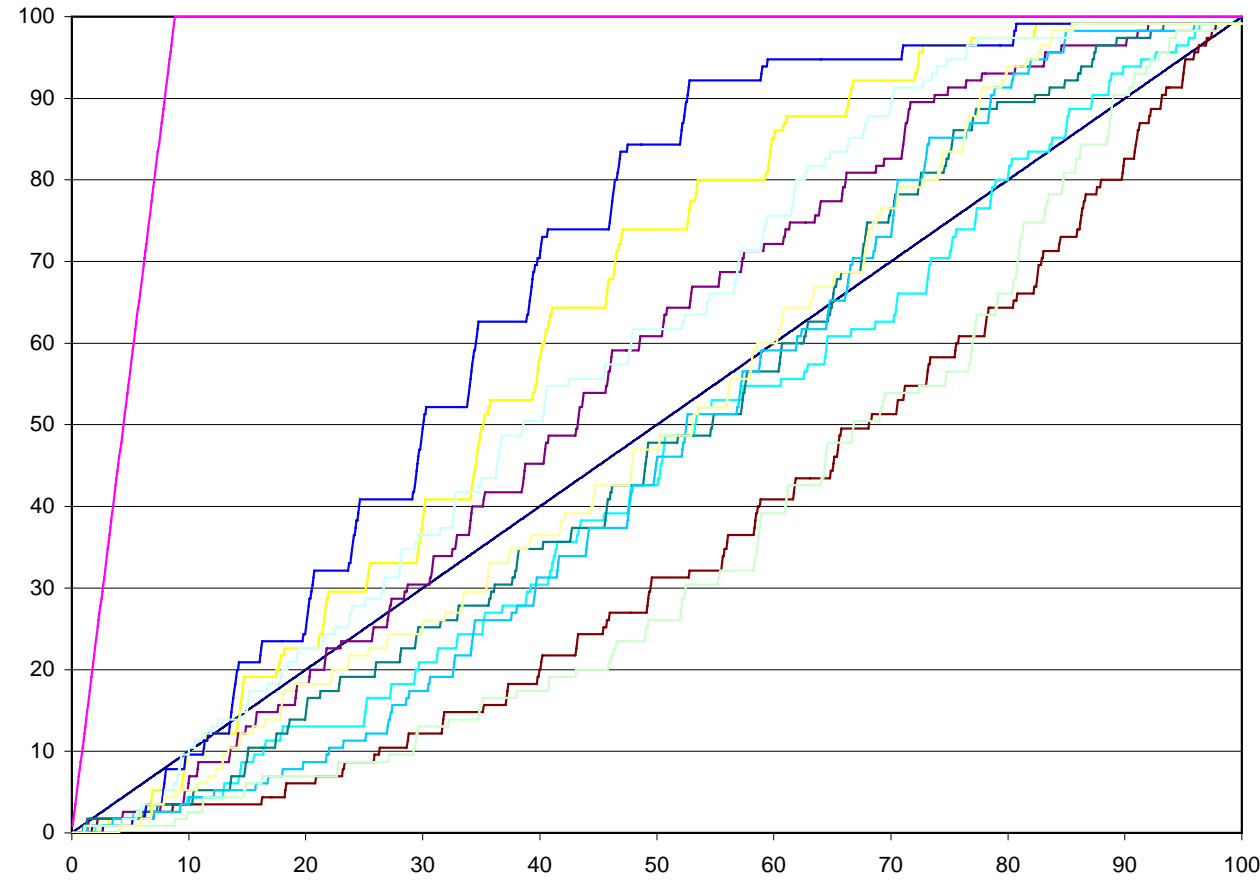

\begin{tabular}{|l|}
\hline - \\
Random \\
Ideal \\
Dock, A \\
Dock/PLP, A \\
- Dock/PMF, A \\
- PLP, A \\
\hline PMF, A \\
- Dock, B \\
\hline Dock/PLP, B \\
Dock/PMF, B \\
PLP, B \\
PMF, B \\
\hline
\end{tabular}

Dockit

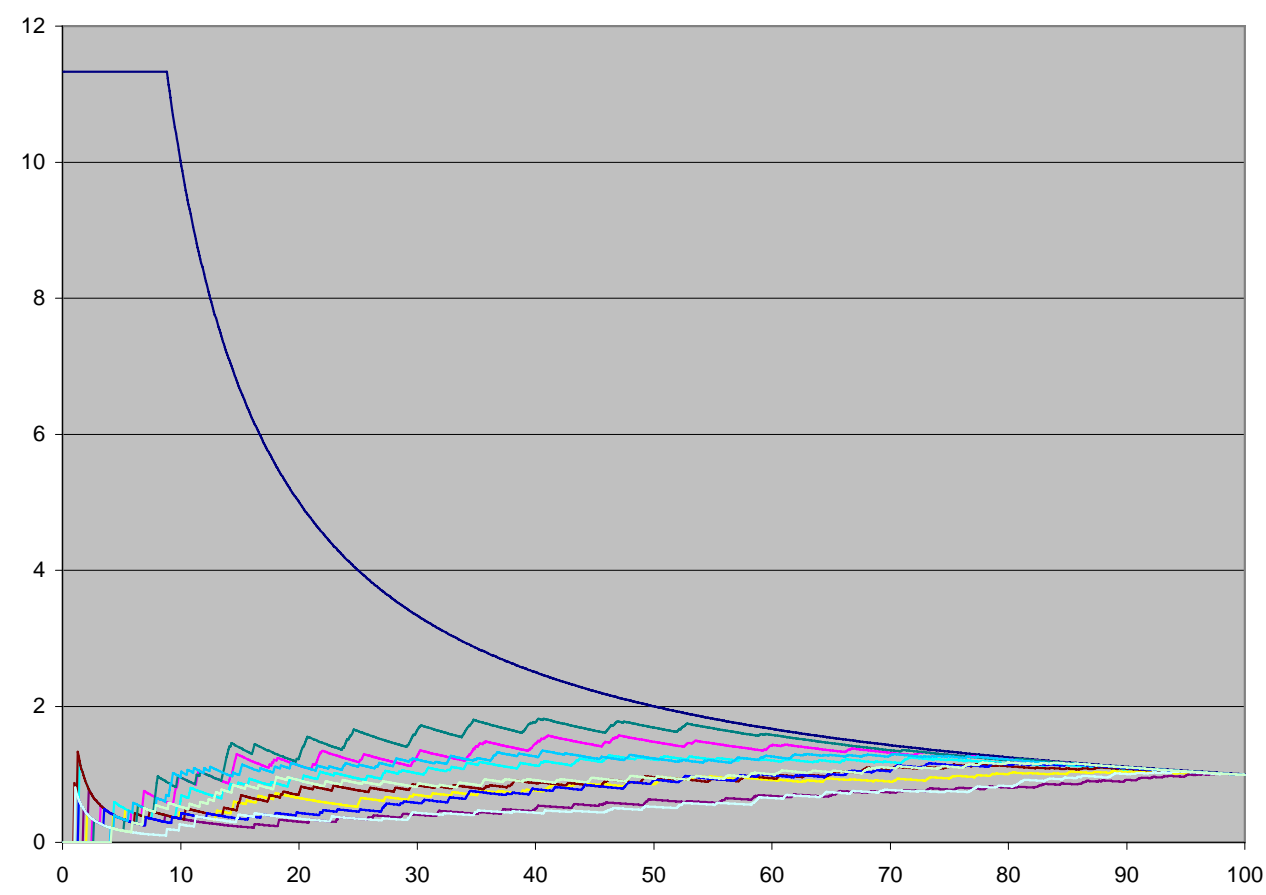

\begin{tabular}{|l|}
\hline - Ideal \\
- Dock, A \\
Dock/PLP, A \\
- Dock/PMF, A \\
- PLP, A \\
- PMF, A \\
- Docki, B \\
- Dock/PLP, B \\
- Dock/PMF, B \\
PLP, B \\
PMF, B
\end{tabular}


FlexX

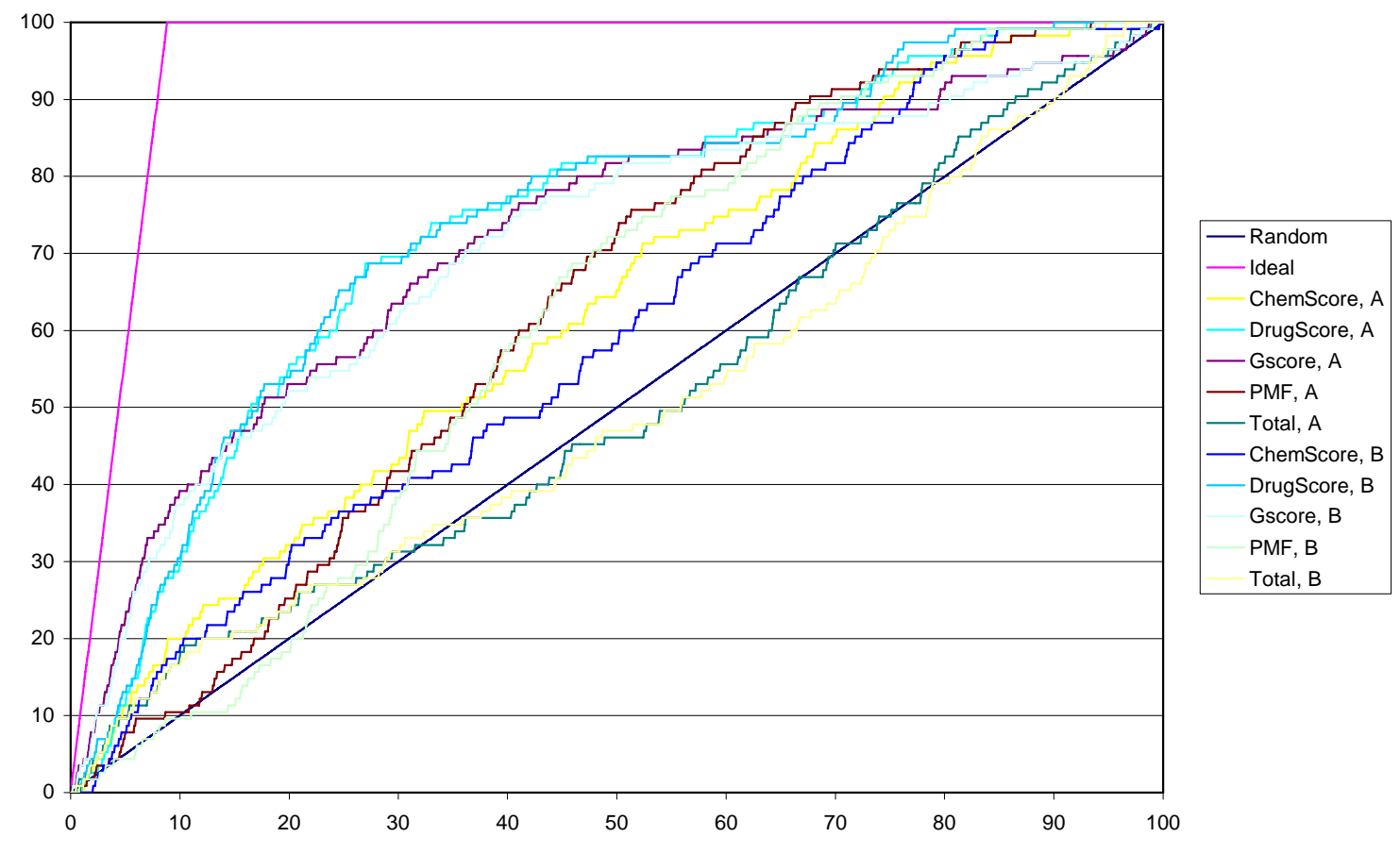

FlexX

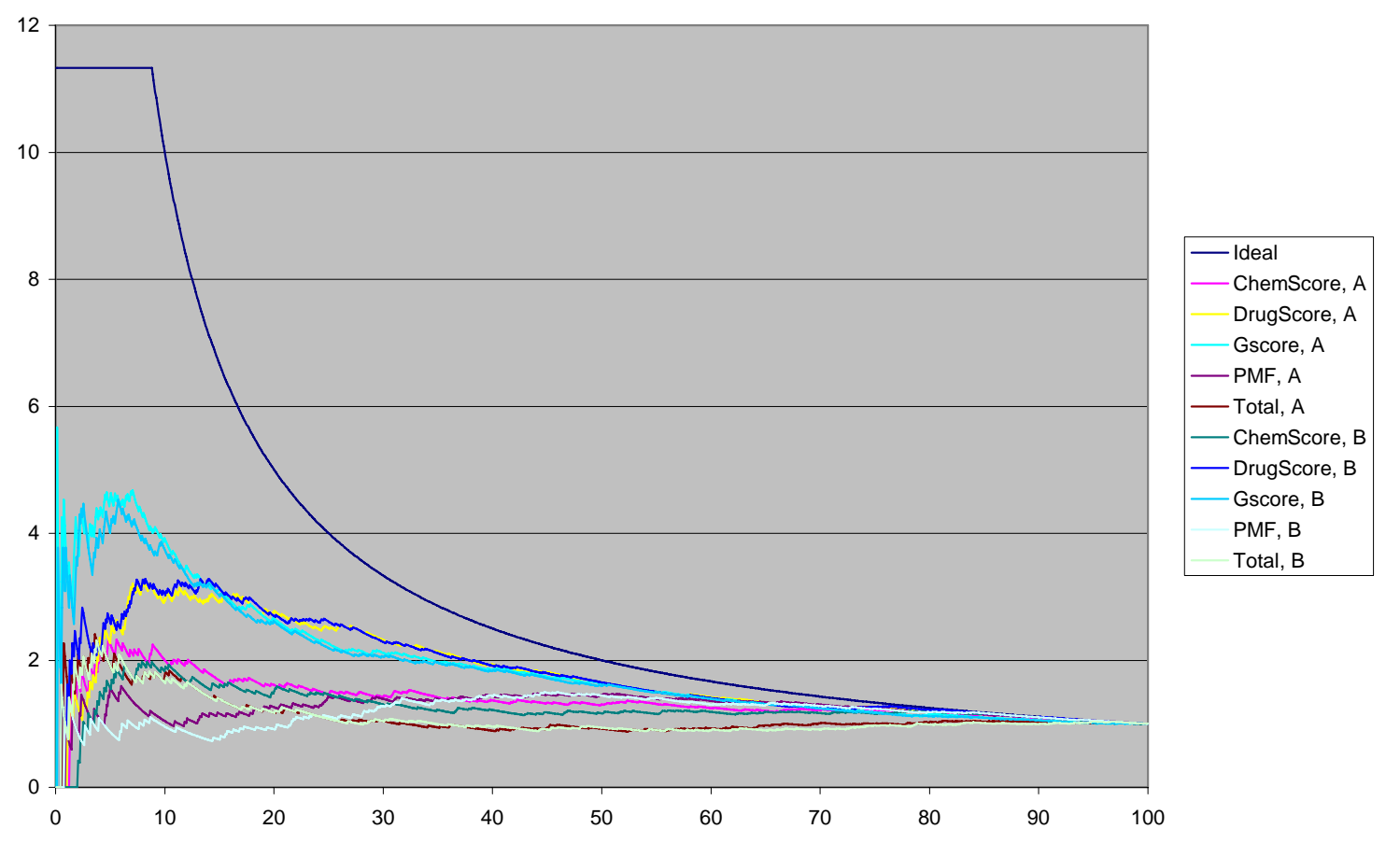




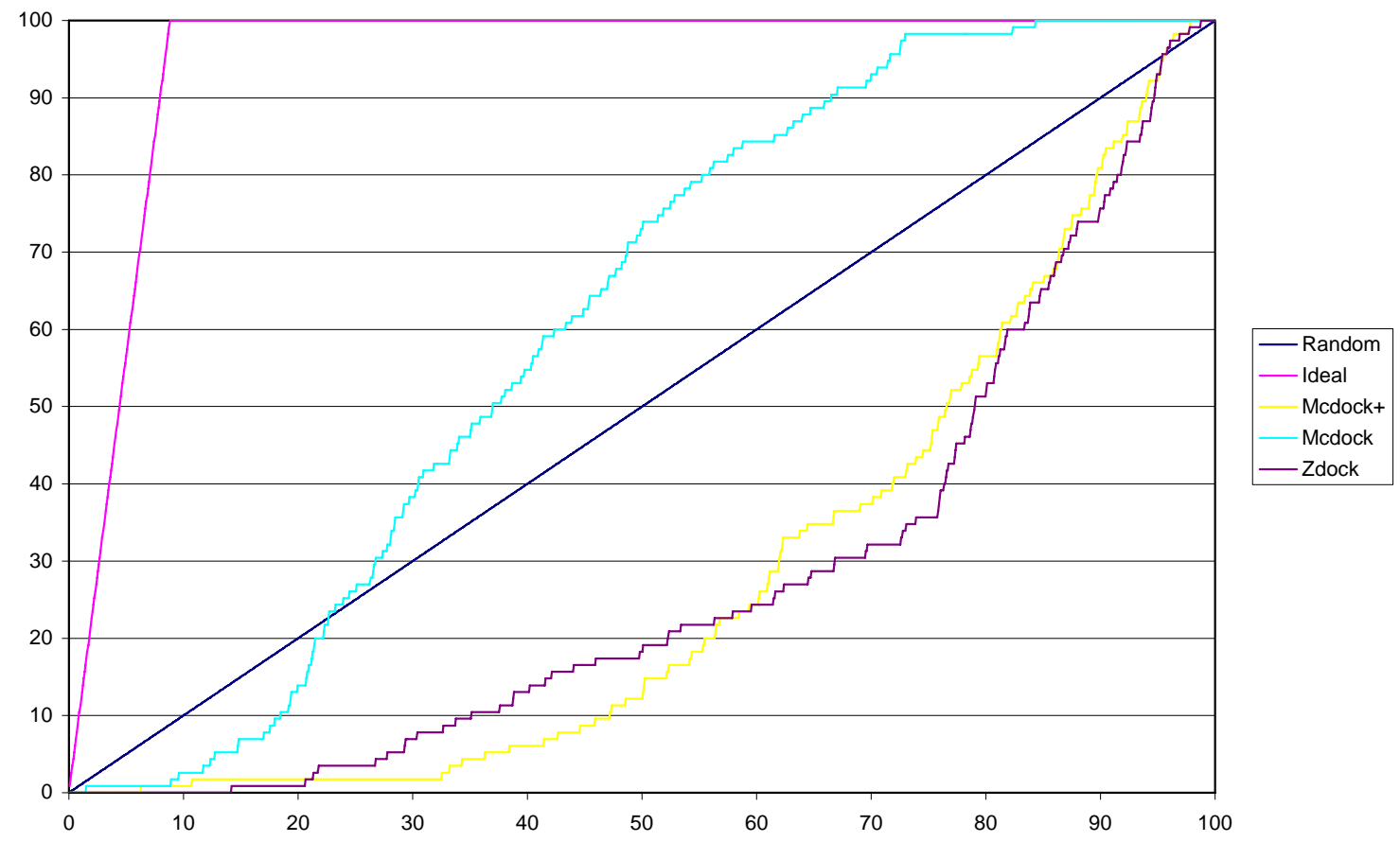

Flo

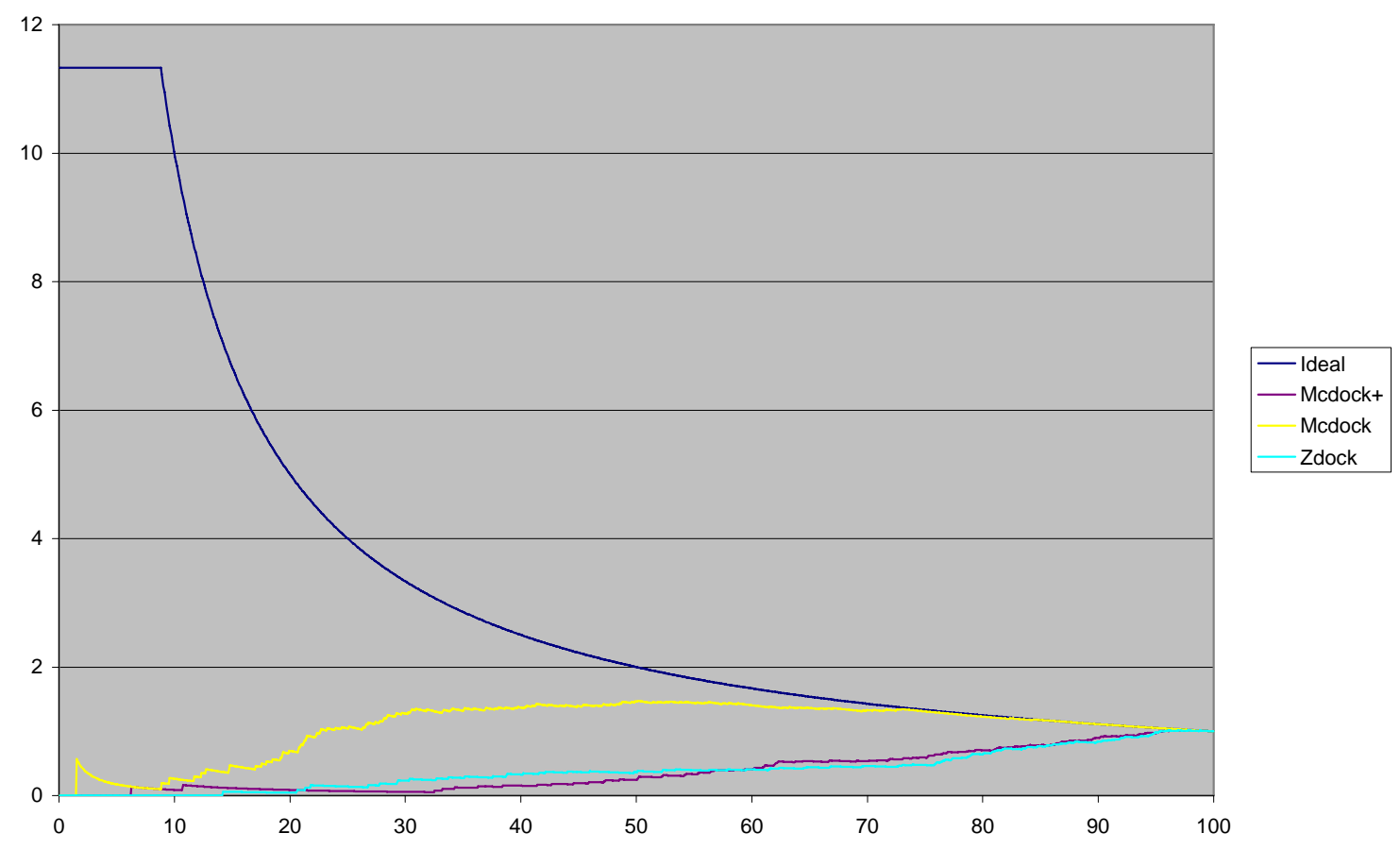


Fred

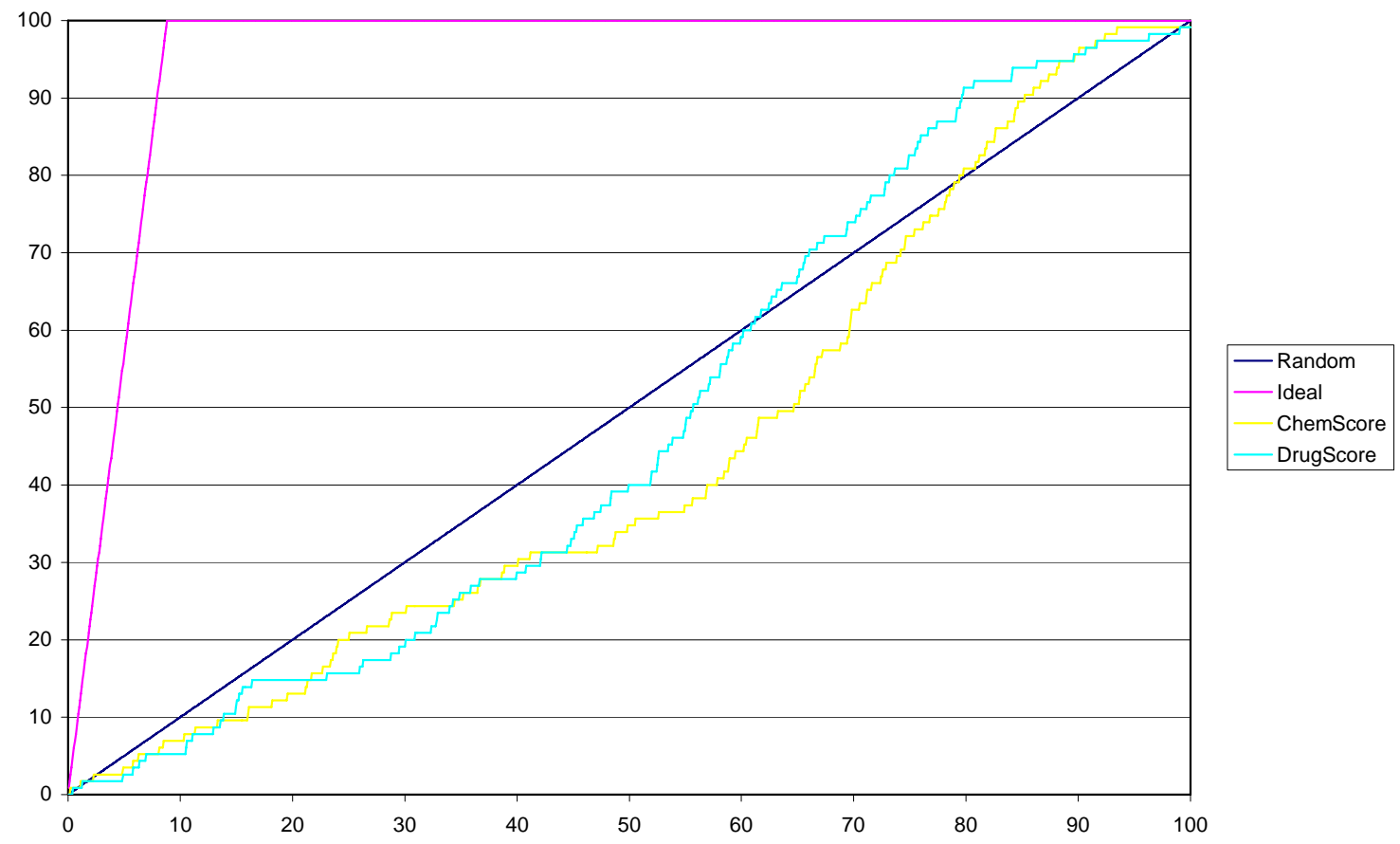

Fred

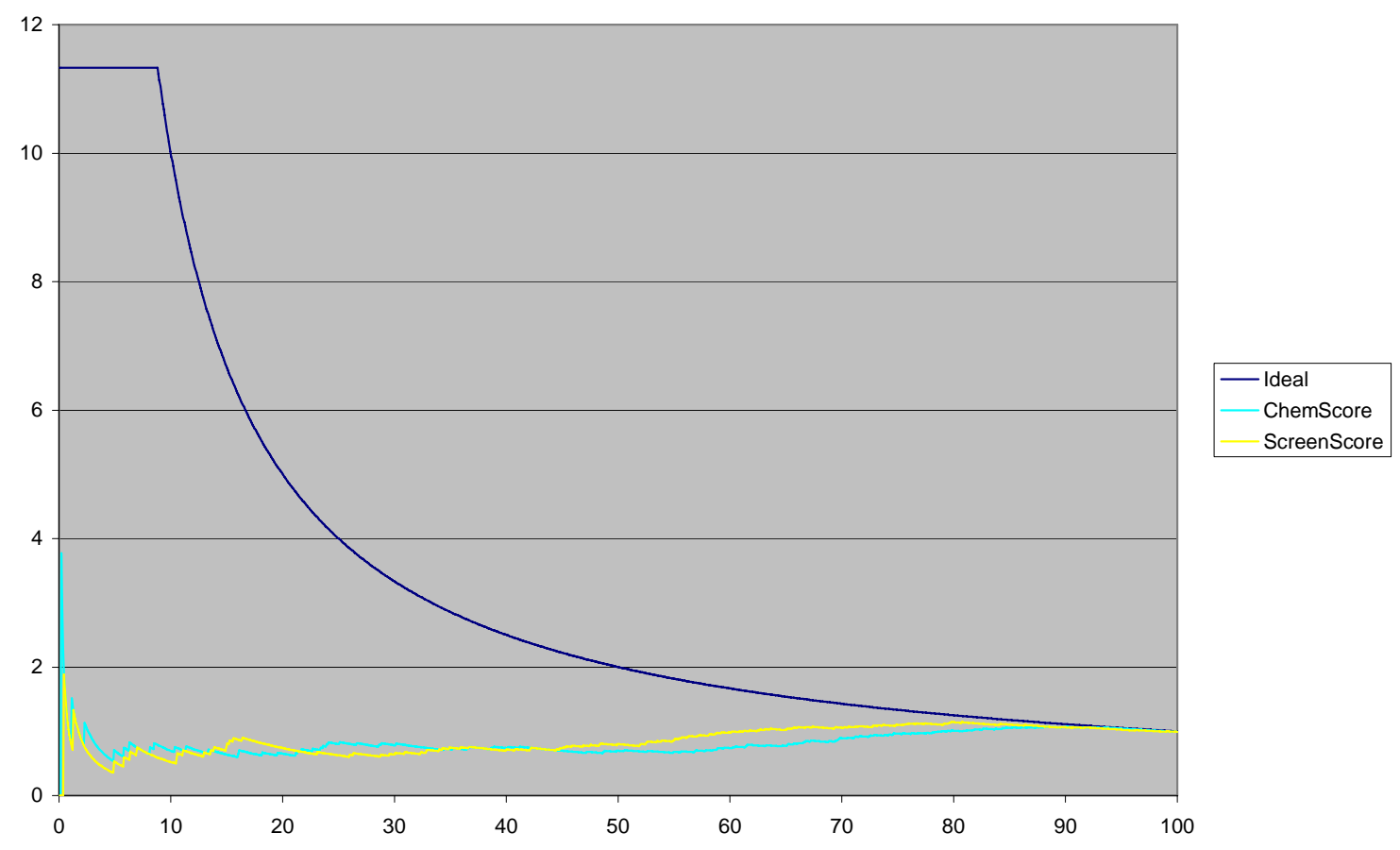




\section{Glide}

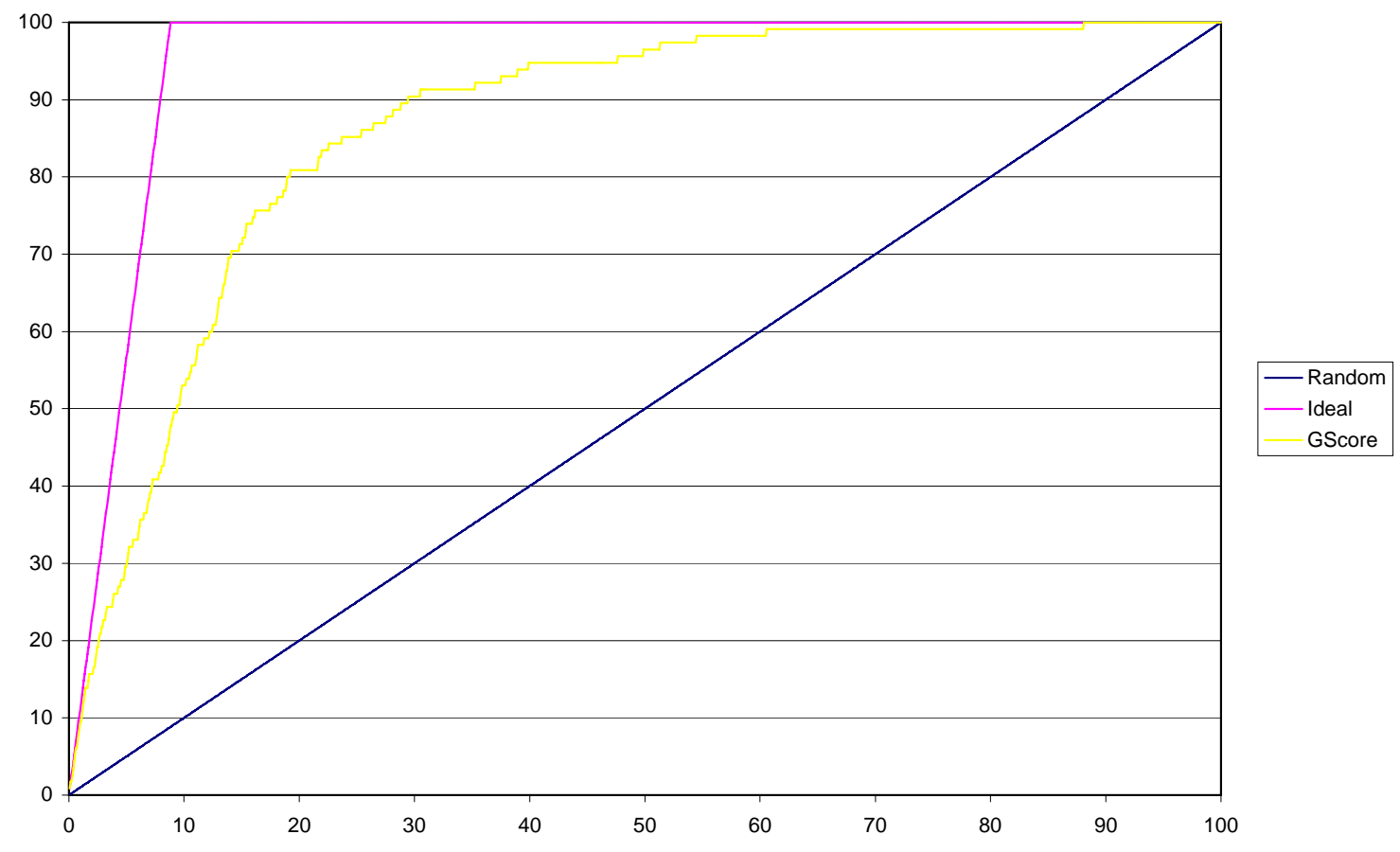

Glide

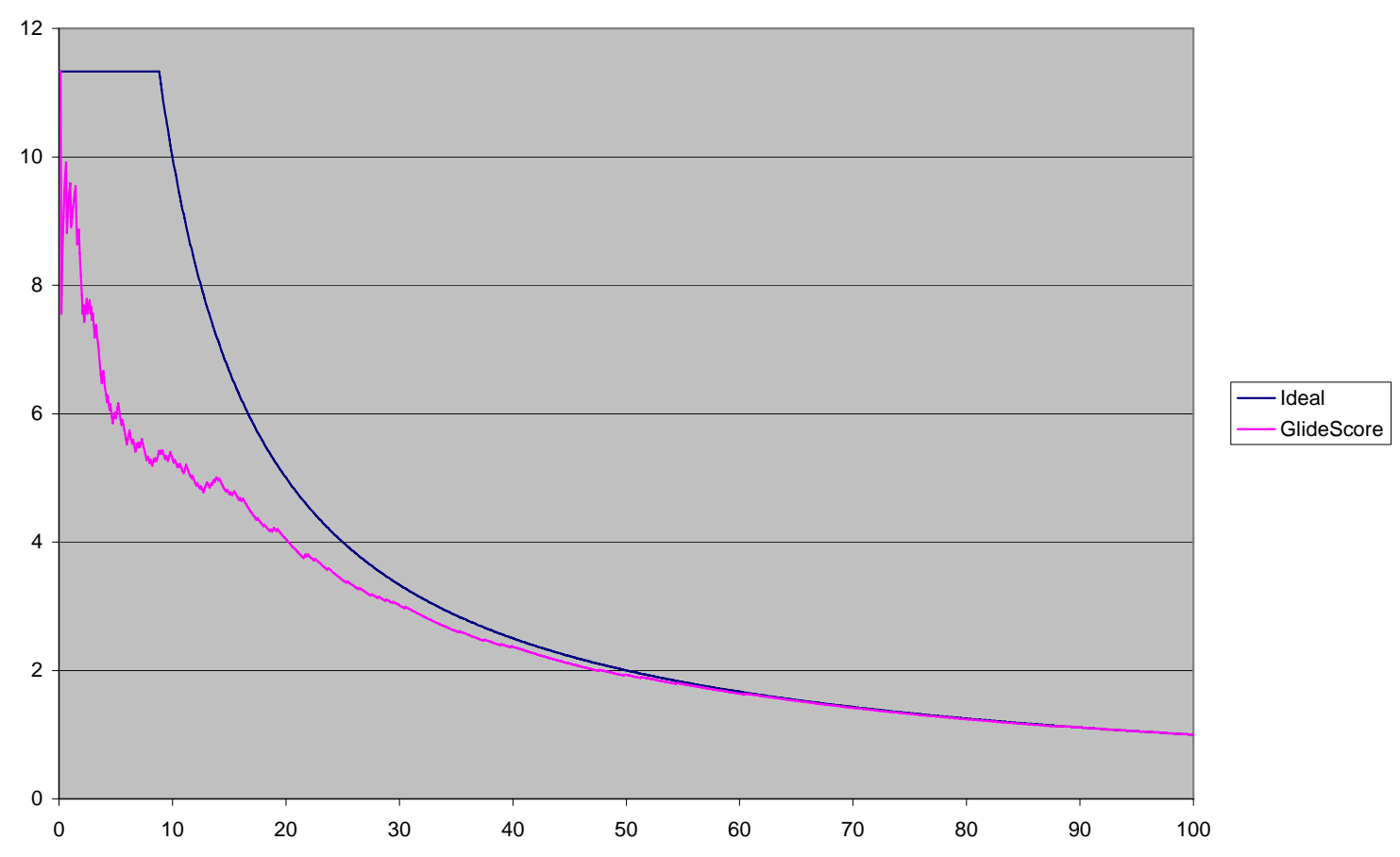


Gold

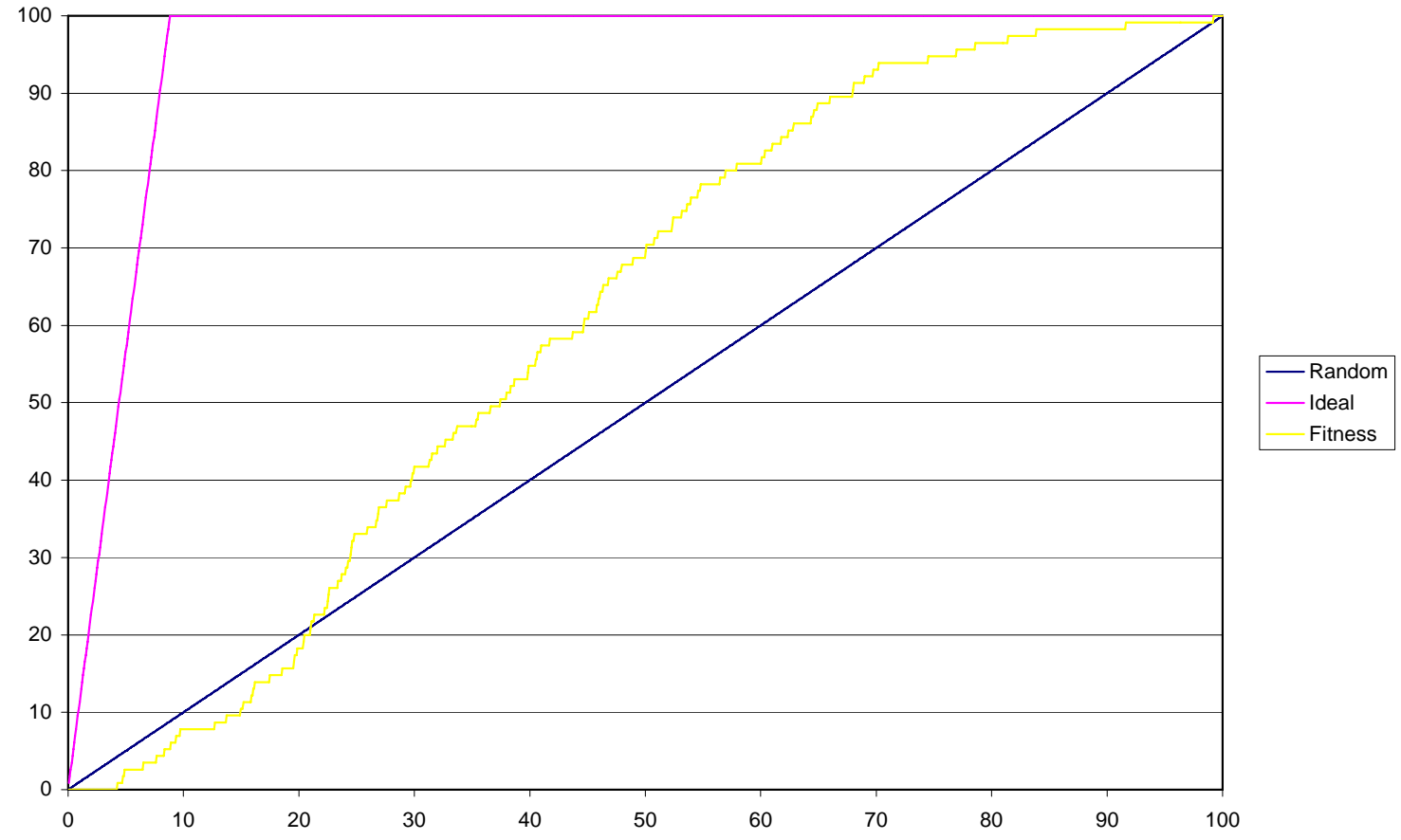

Gold

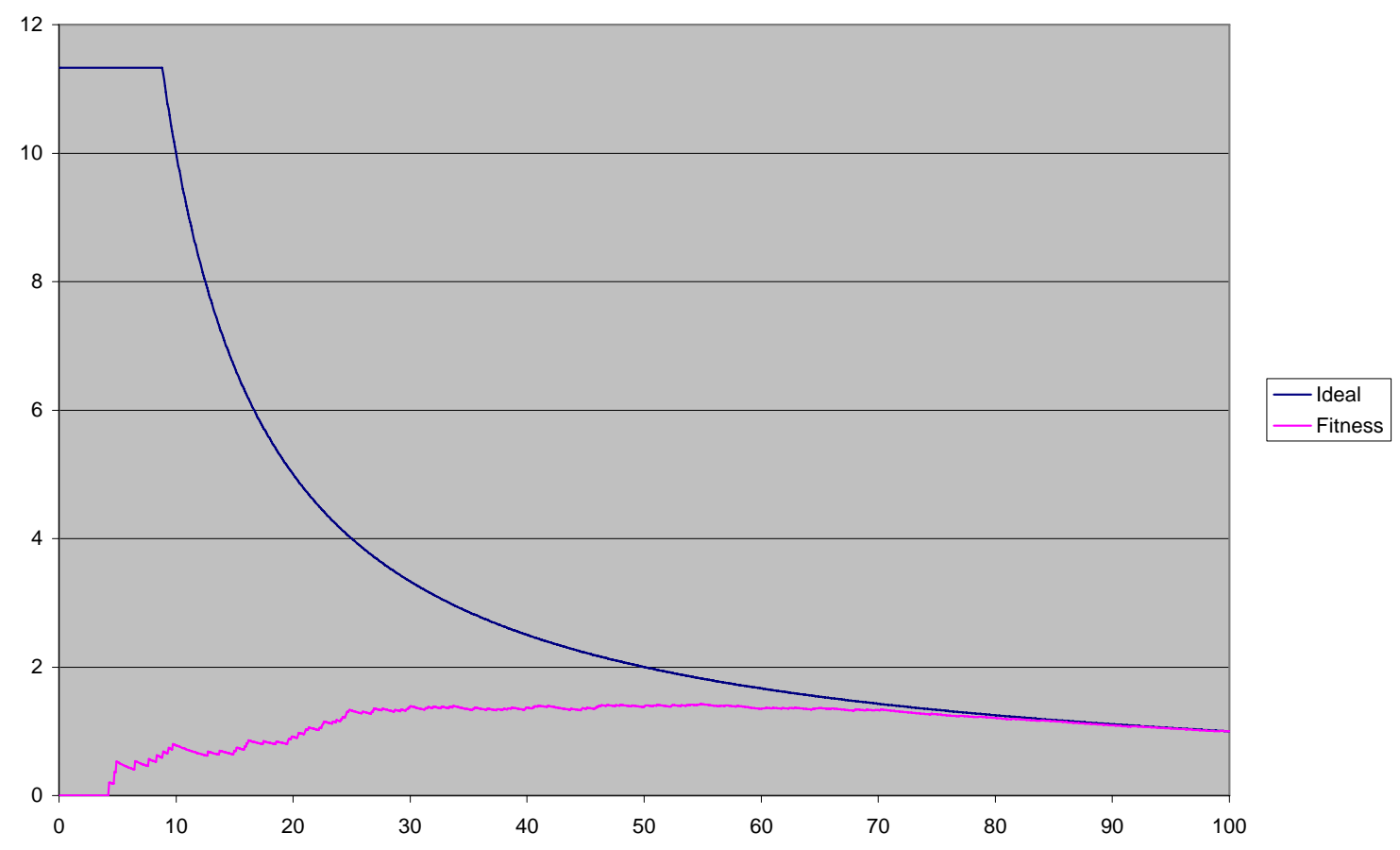




\section{LigFit}

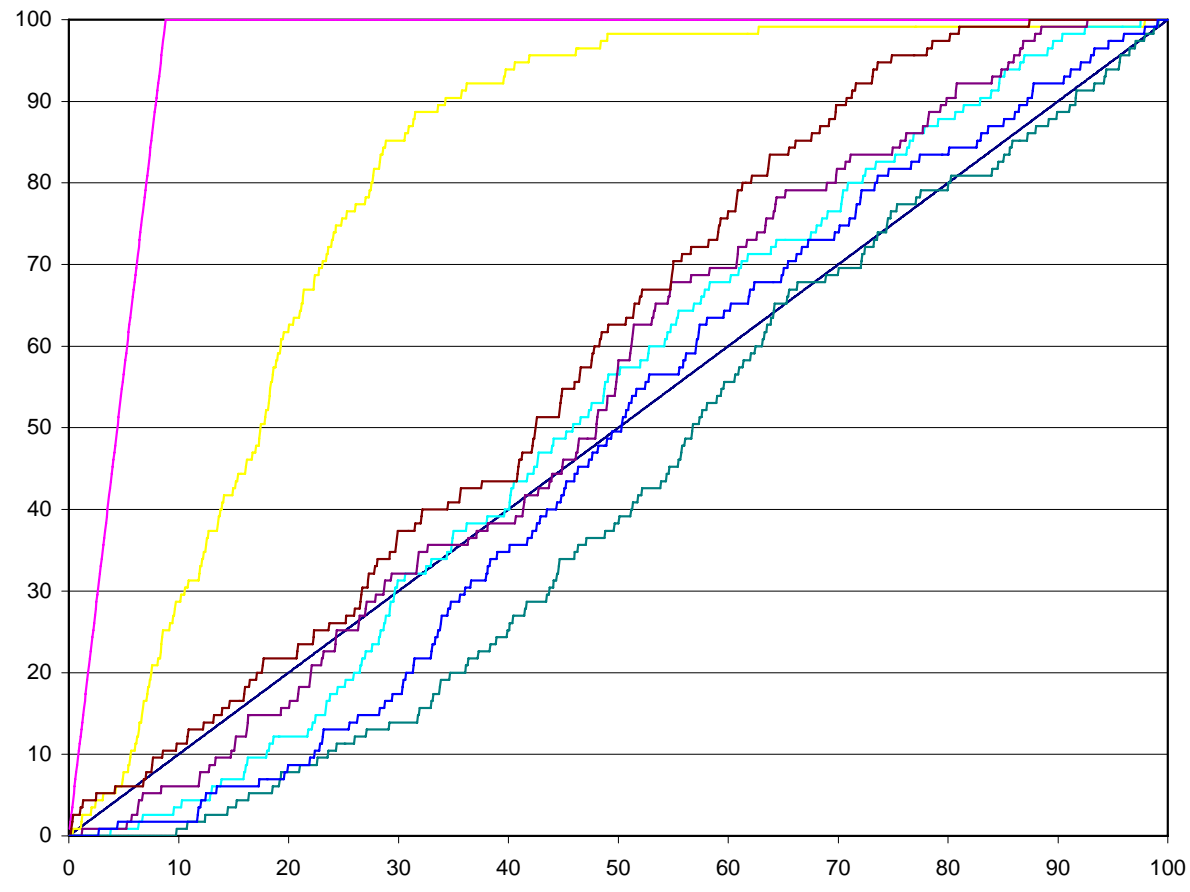

\begin{tabular}{|l}
\hline - Random \\
- Ideal \\
DrugScore, CFF \\
DrugScore, Dreiding \\
- Ligscore2, CFF \\
Ligscore2, Dreiding \\
PMF, CFF \\
PMF, Dreiding
\end{tabular}

LigFit

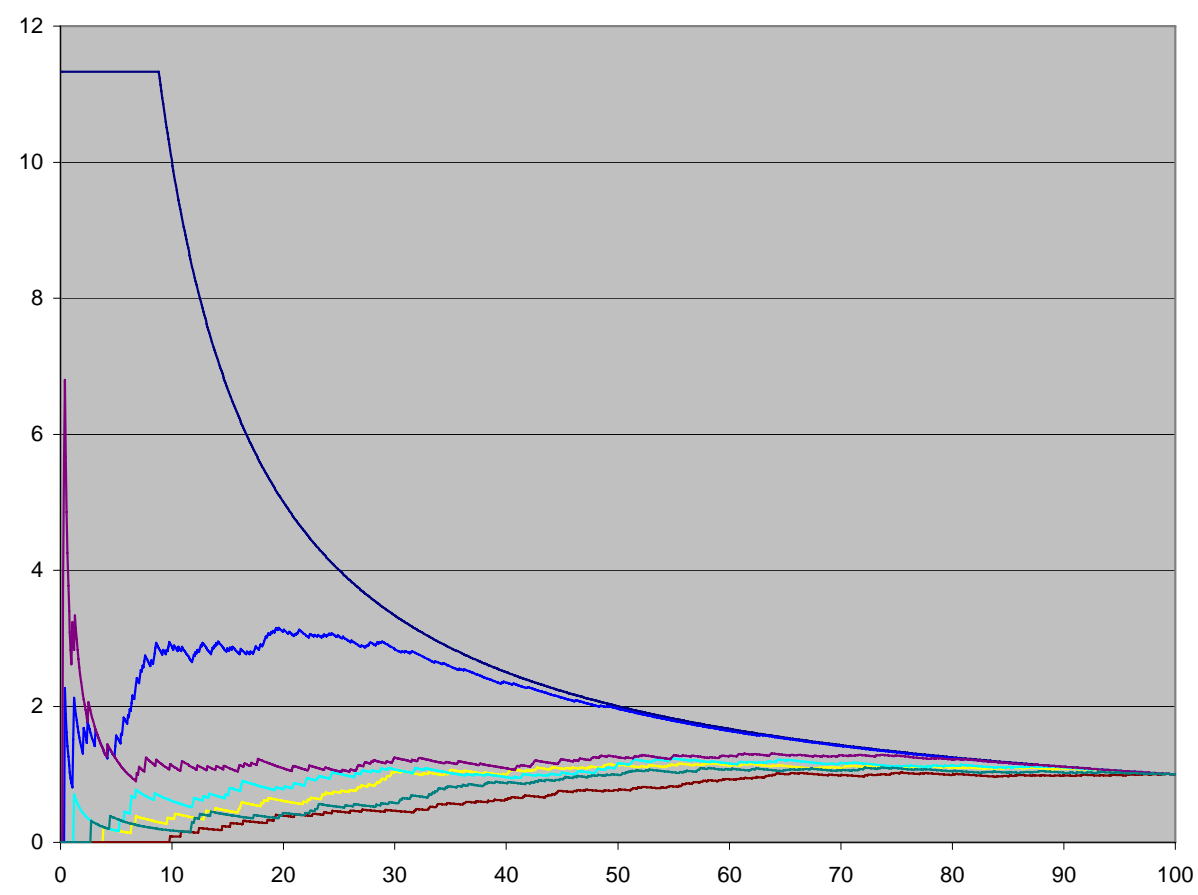

— Ligscore2, Dreiding - PMF, CFF

- PMF, Dreiding 
MOE

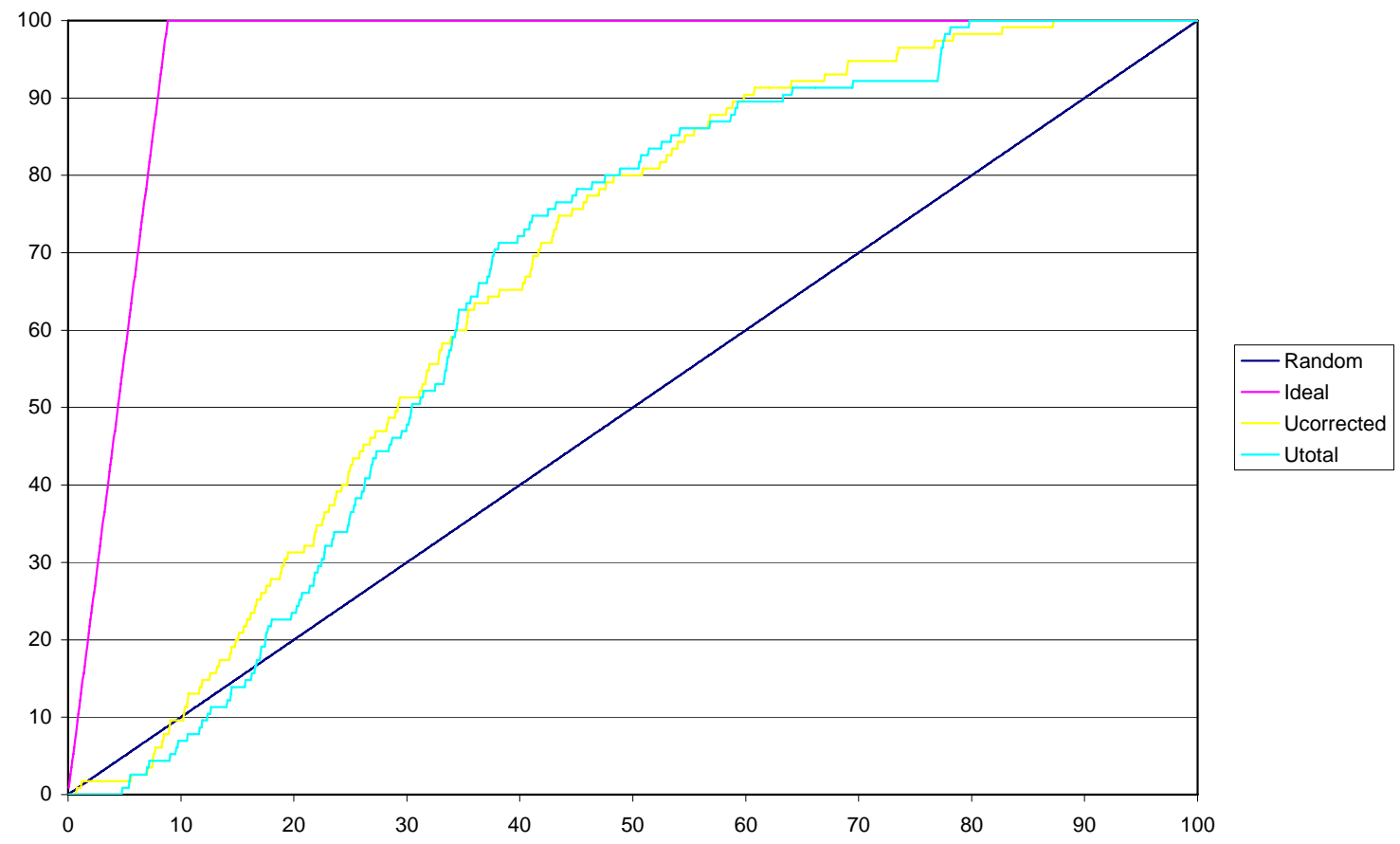

MOE

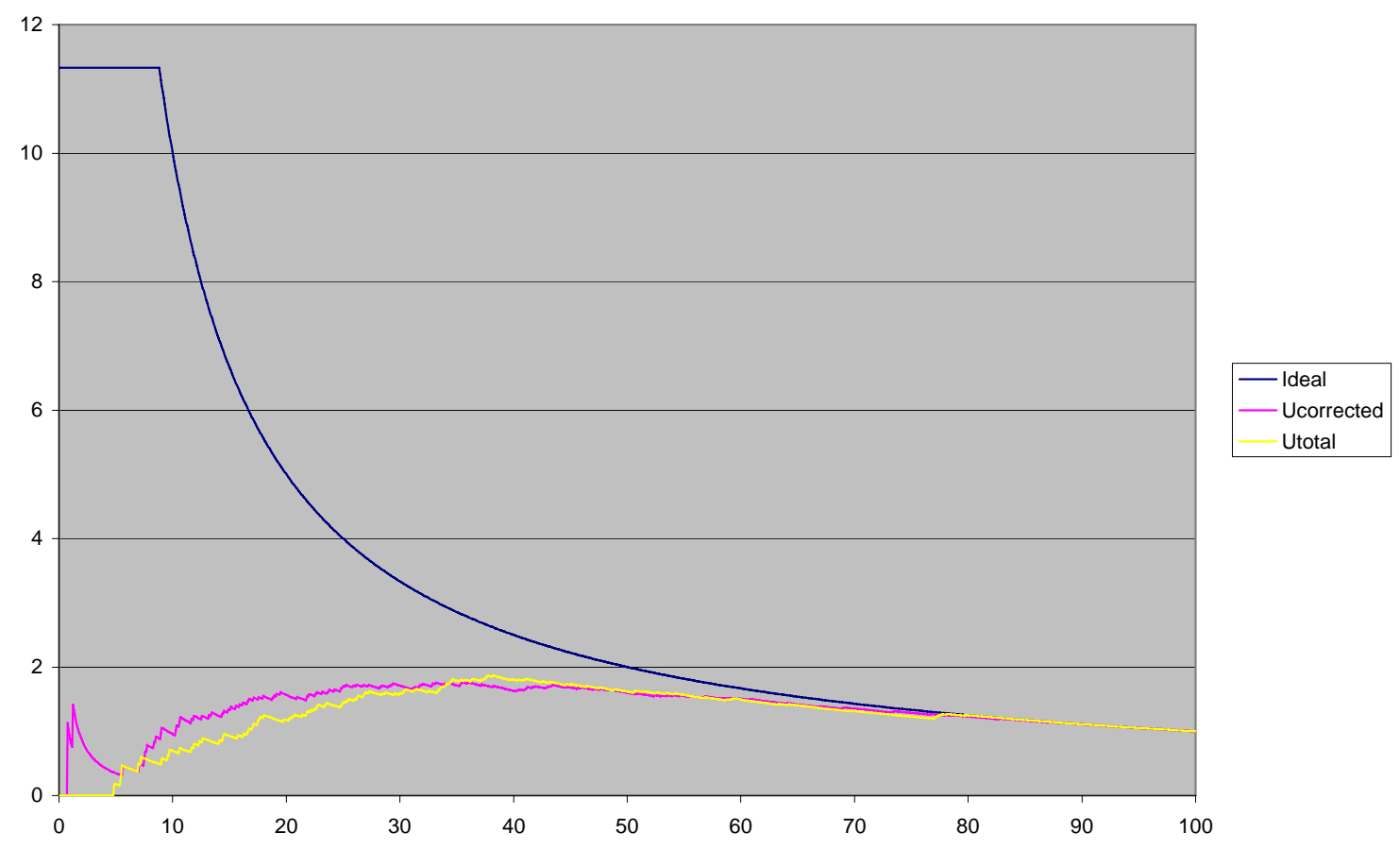


MVP

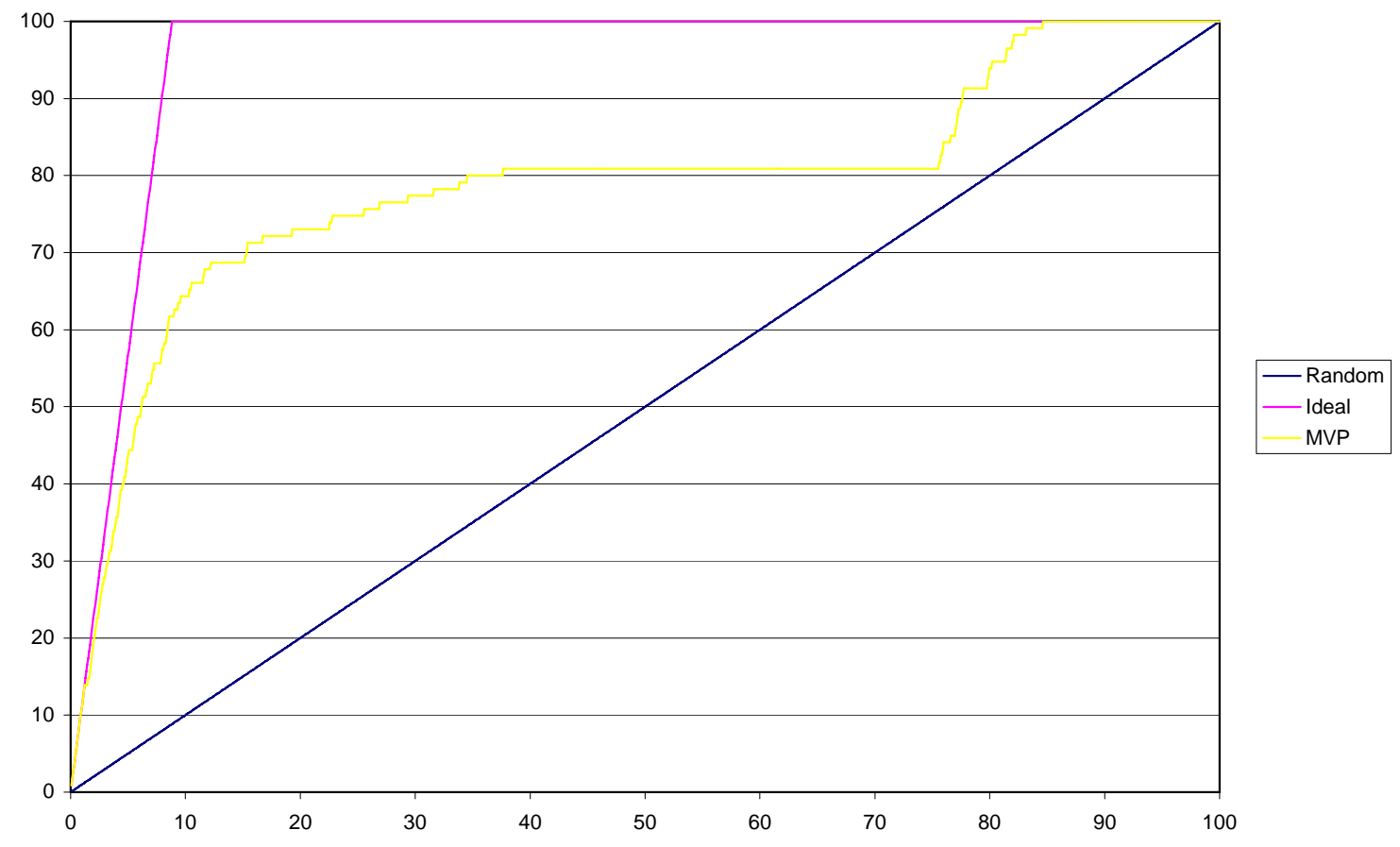


Dock4

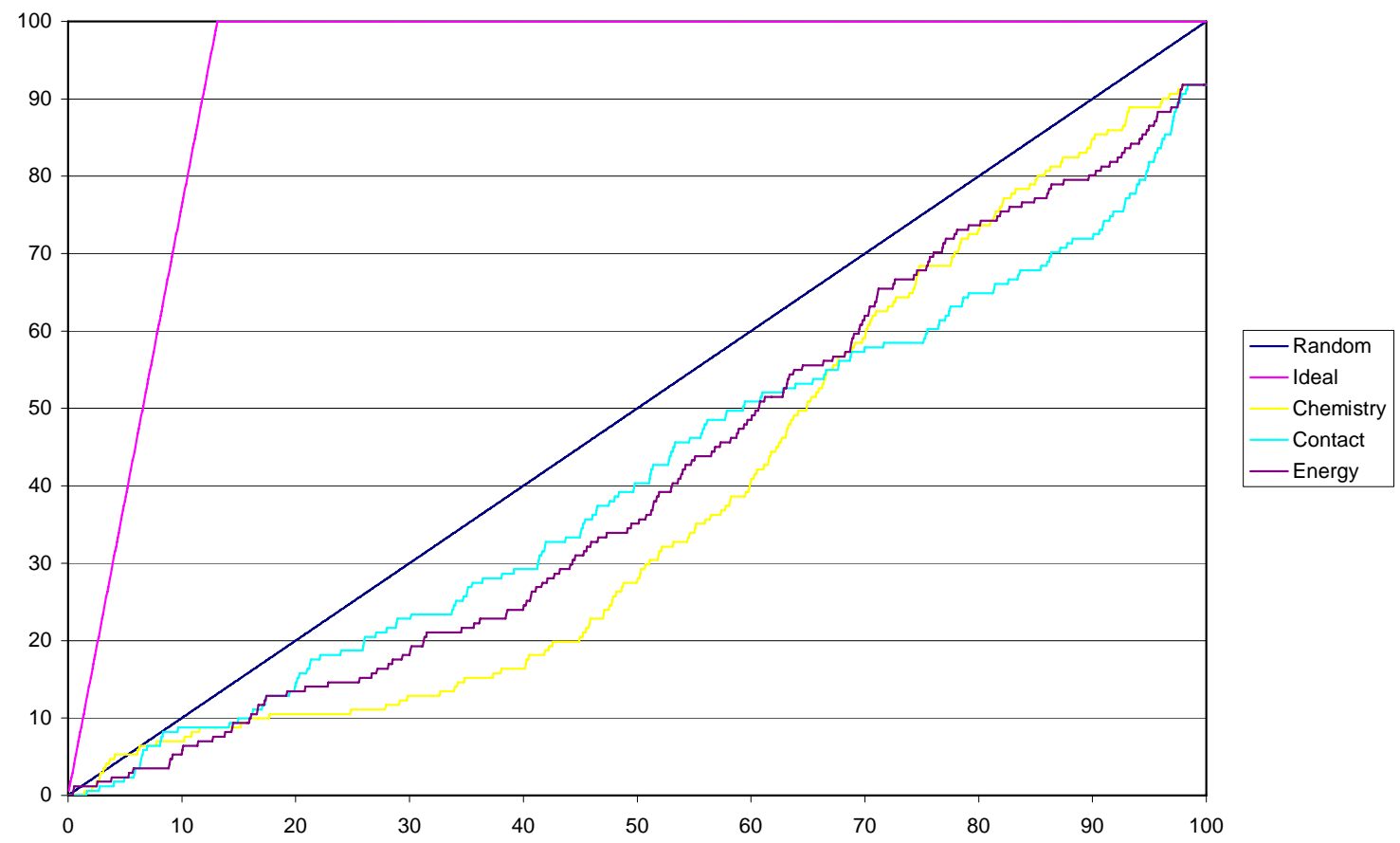

Dock4

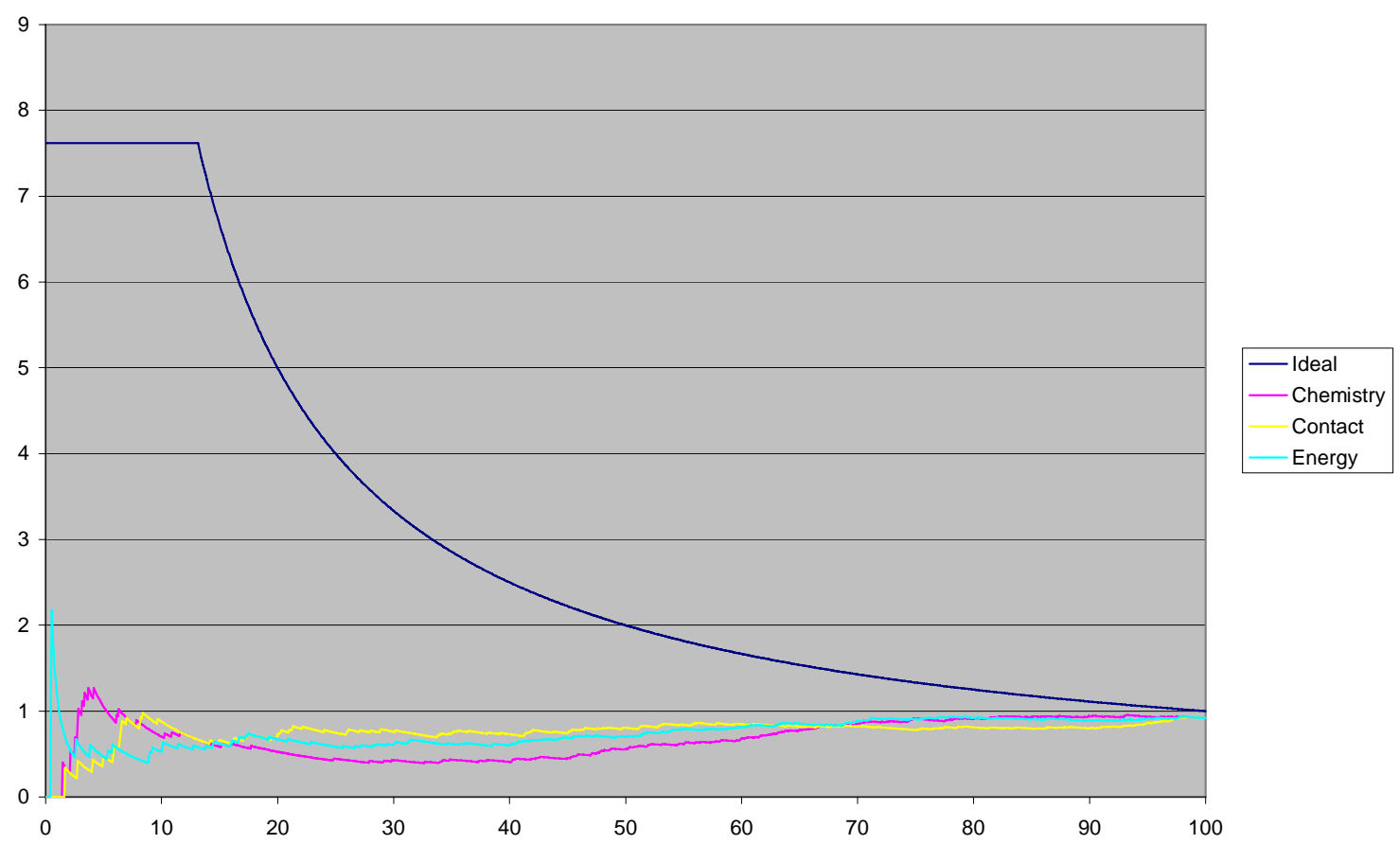




\section{Dockit}

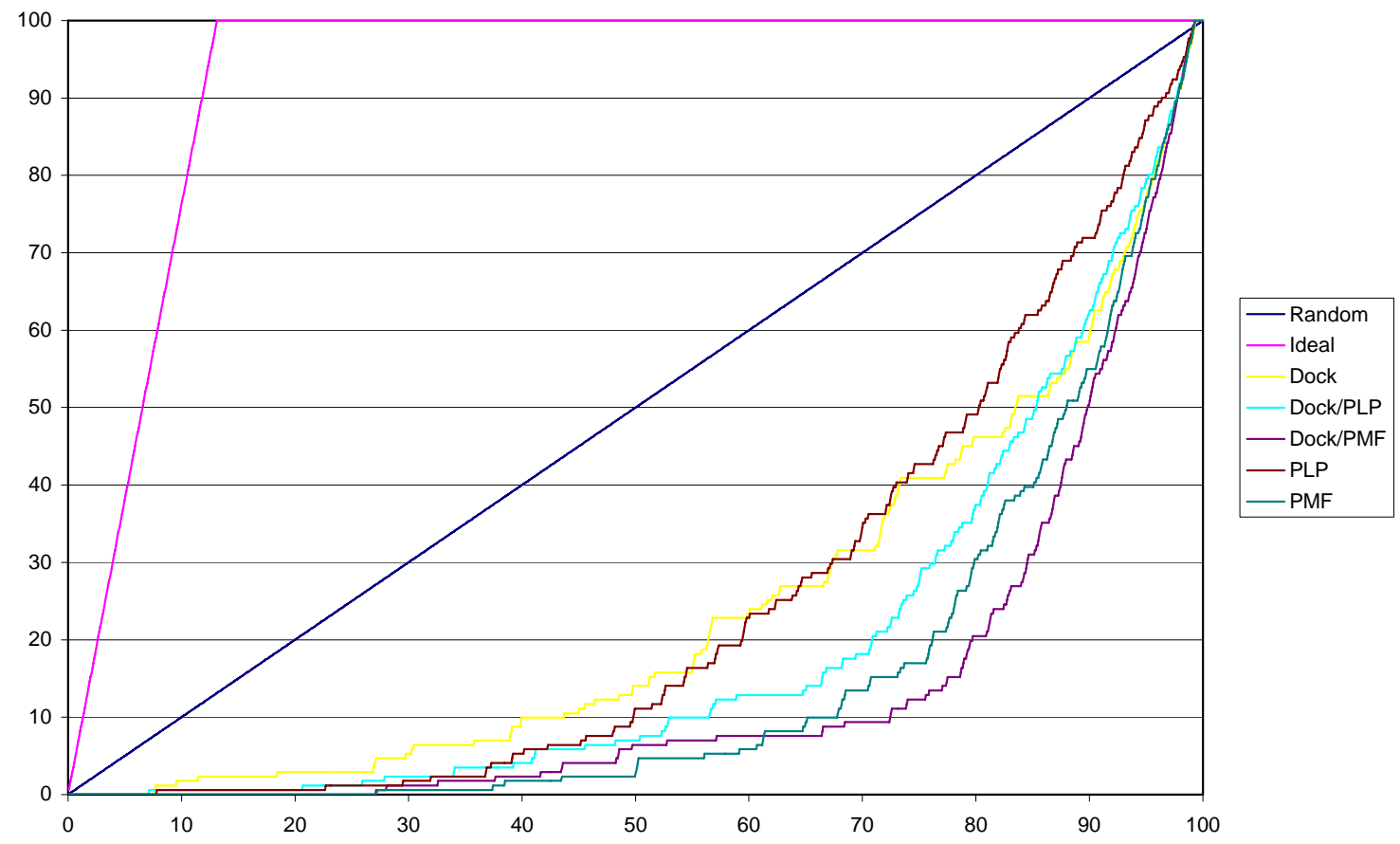

Dockit

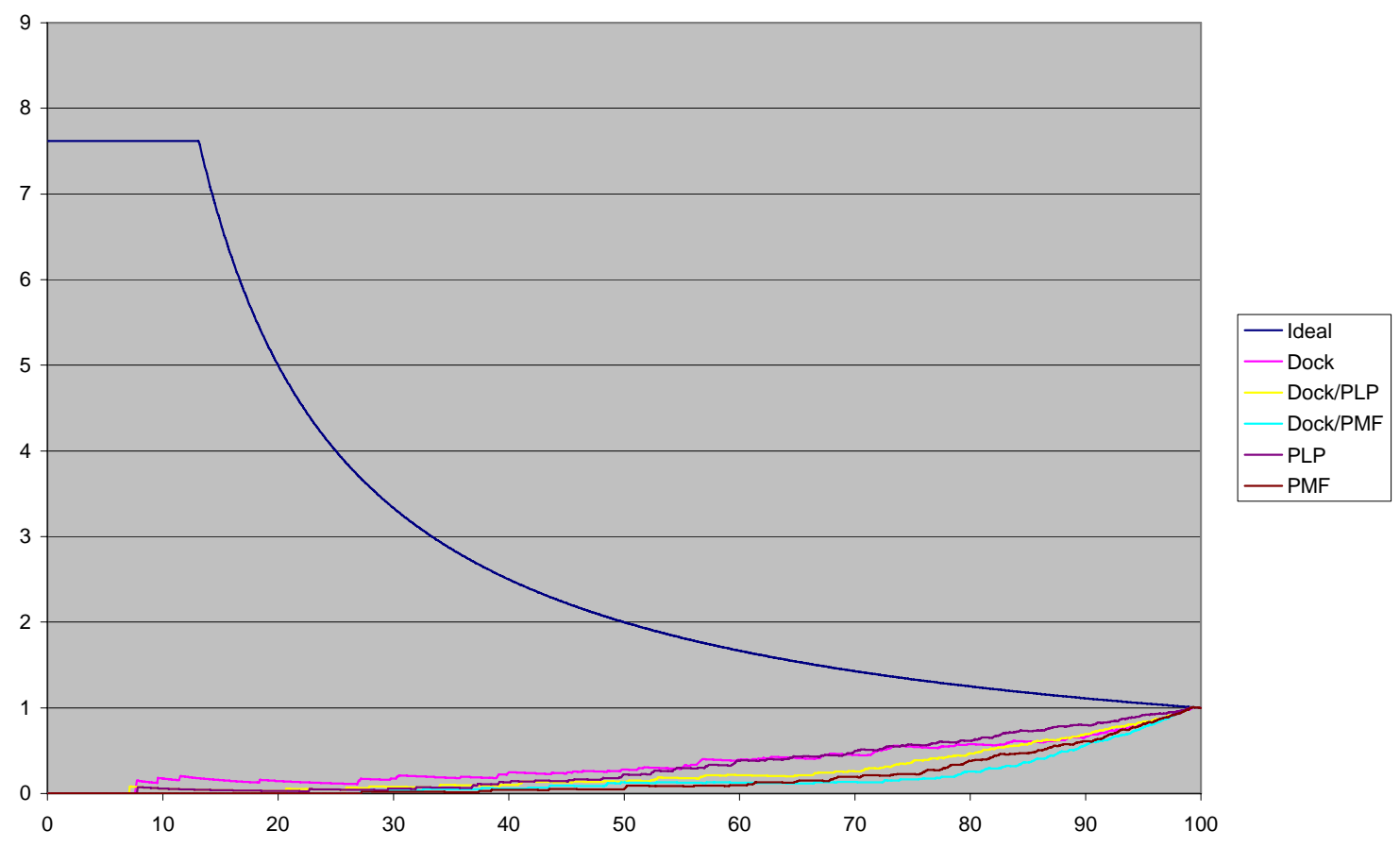


FlexX

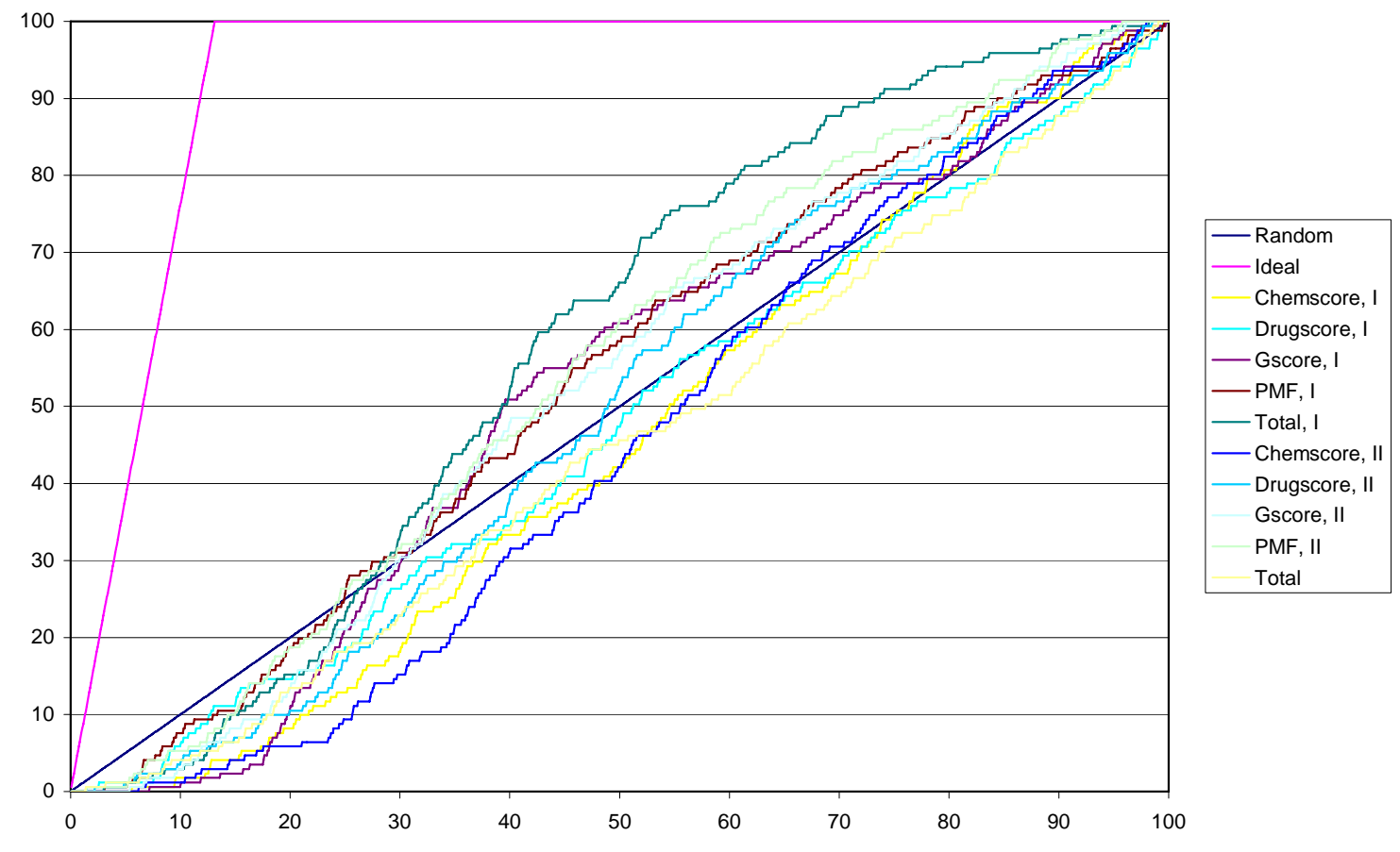

FlexX

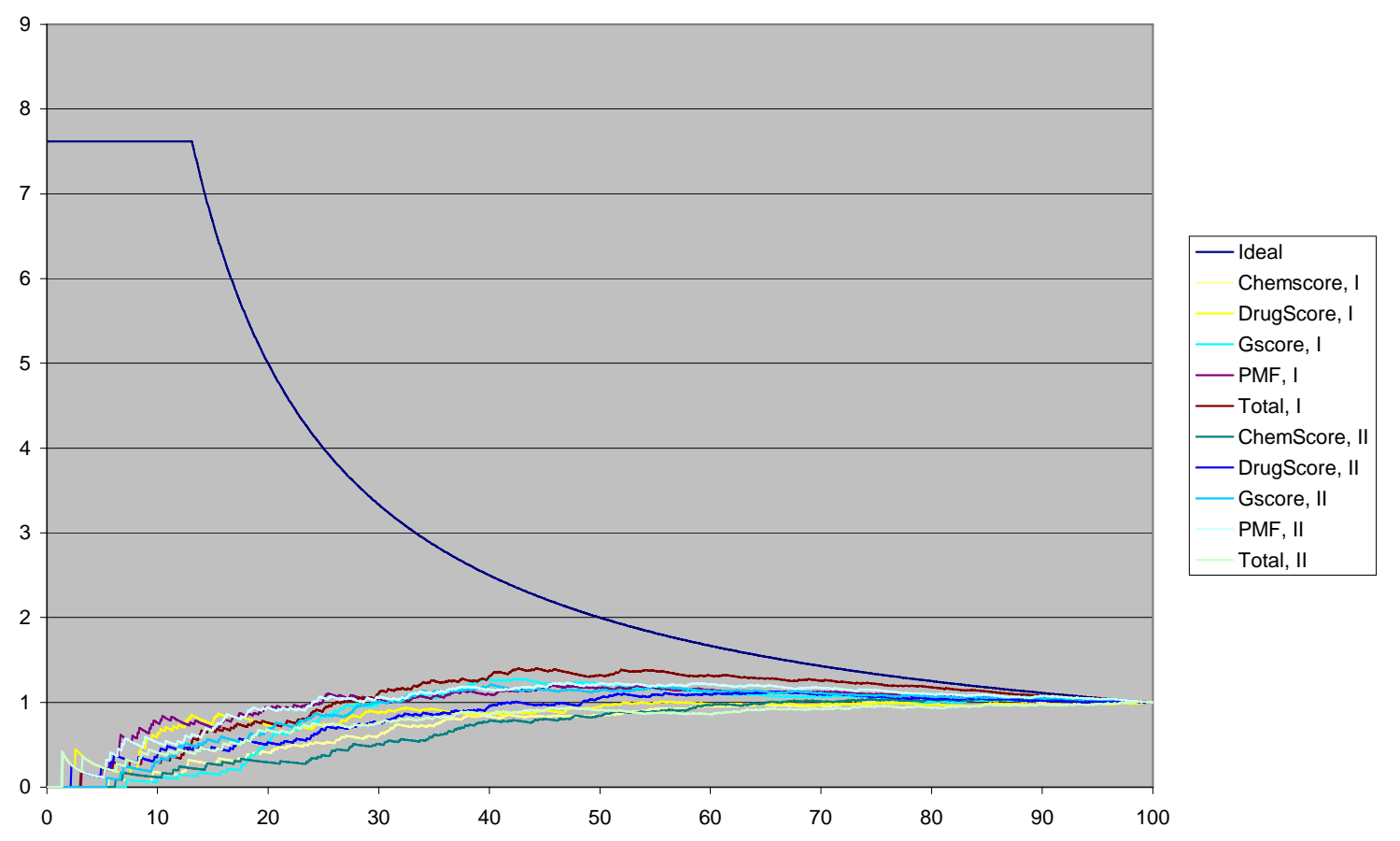




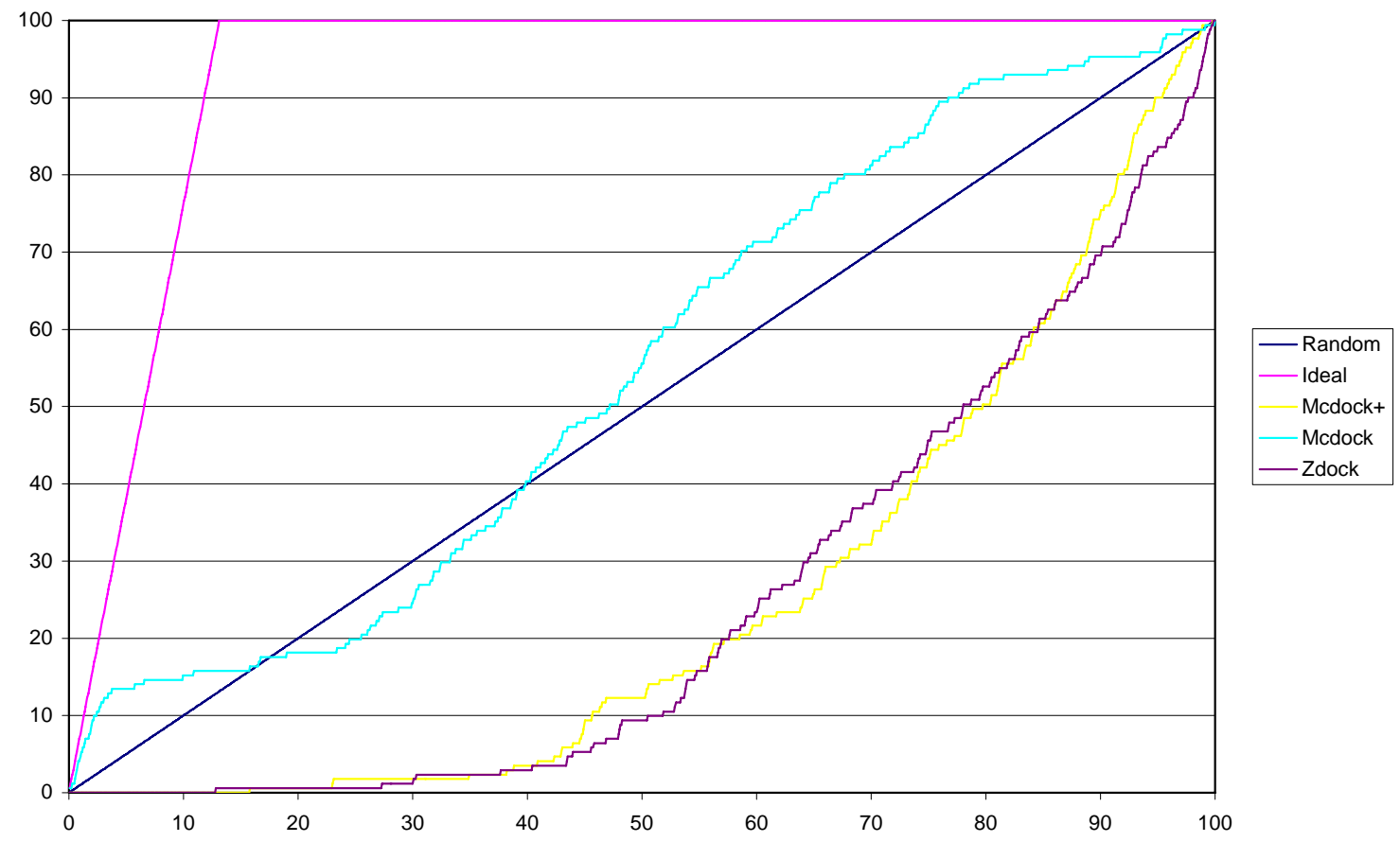

Flo

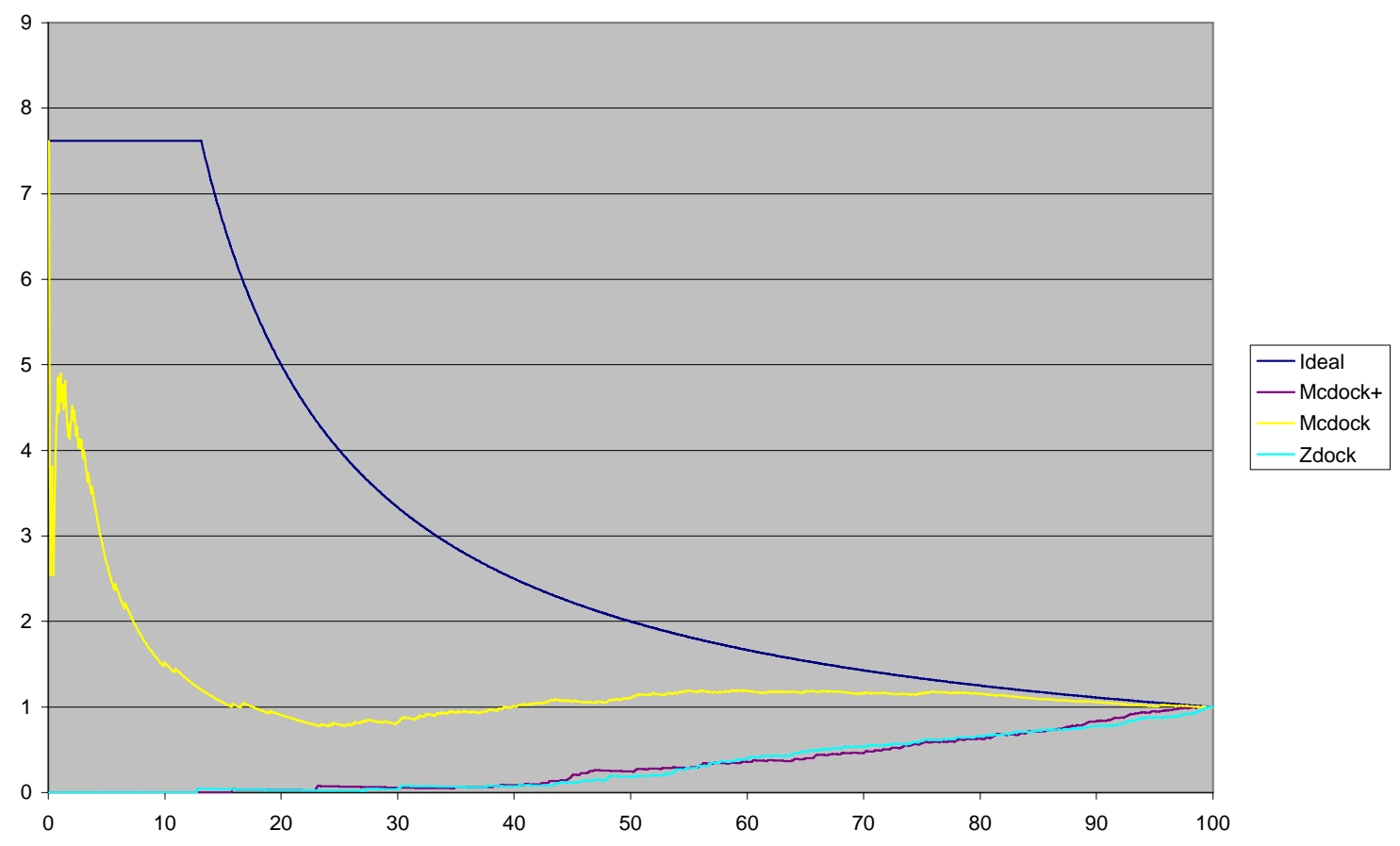


Fred
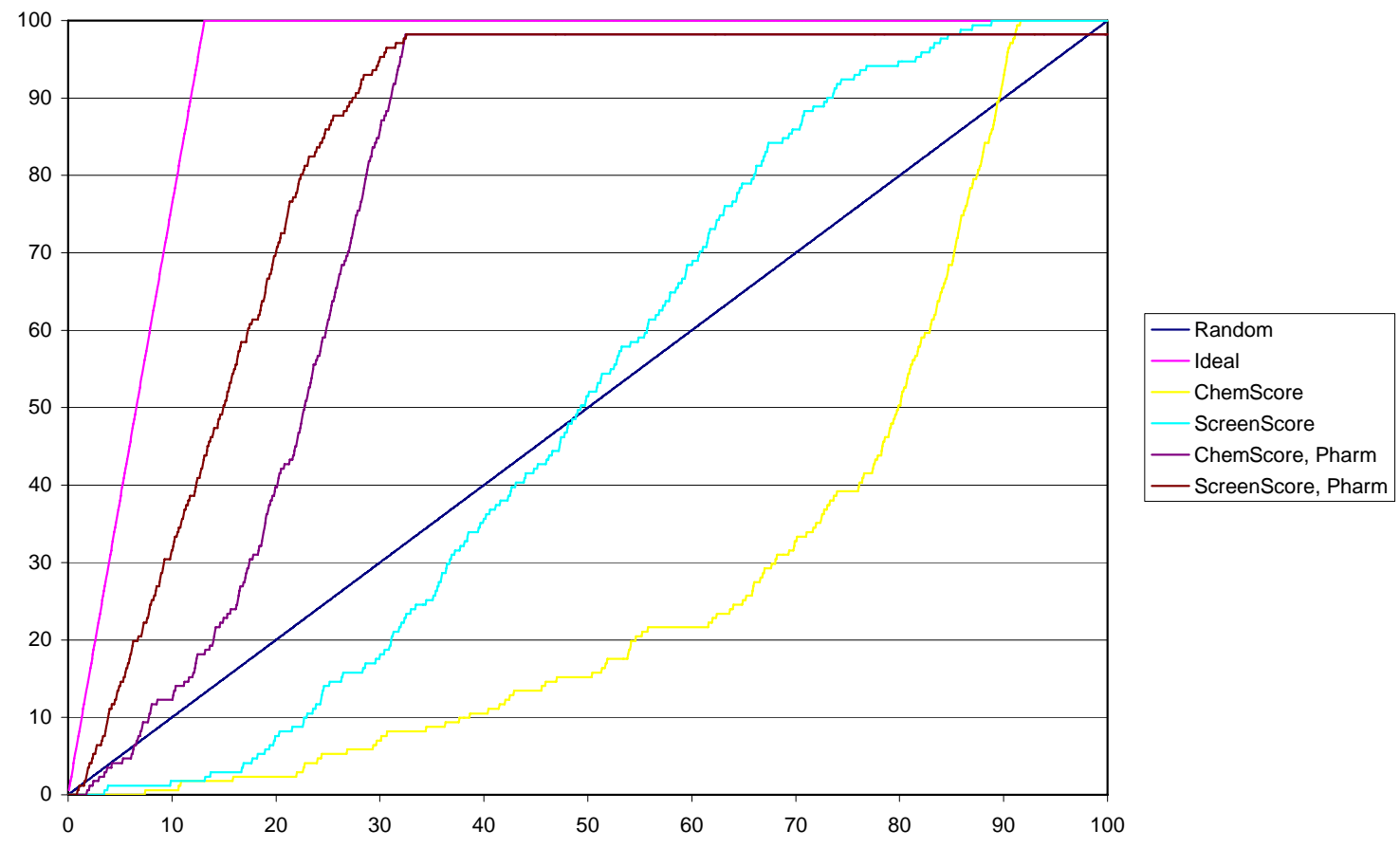

Fred

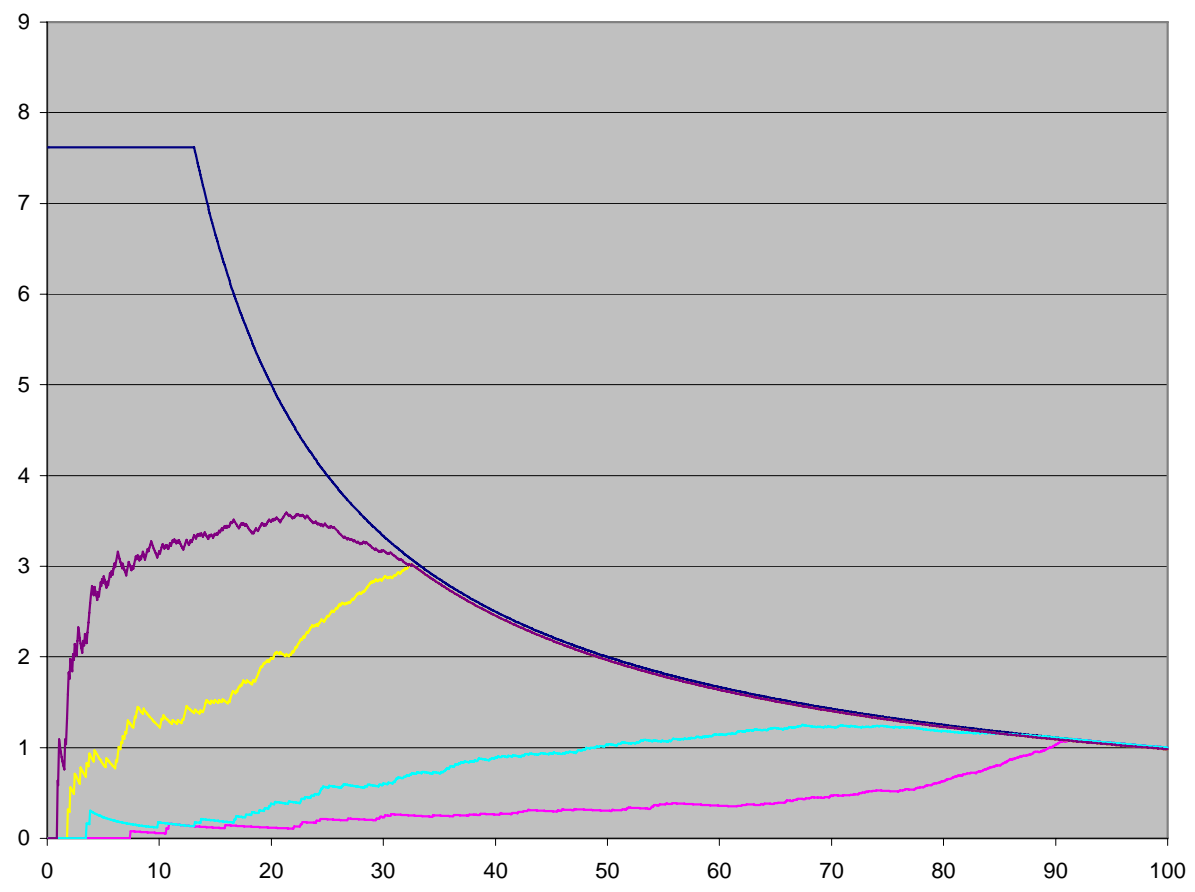

\begin{tabular}{|l|}
\hline - Ideal \\
- ChemScore \\
ChemScore, Phamd \\
- ScreenScore \\
- ScreenScore, Pharm \\
\hline
\end{tabular}




\section{Glide}

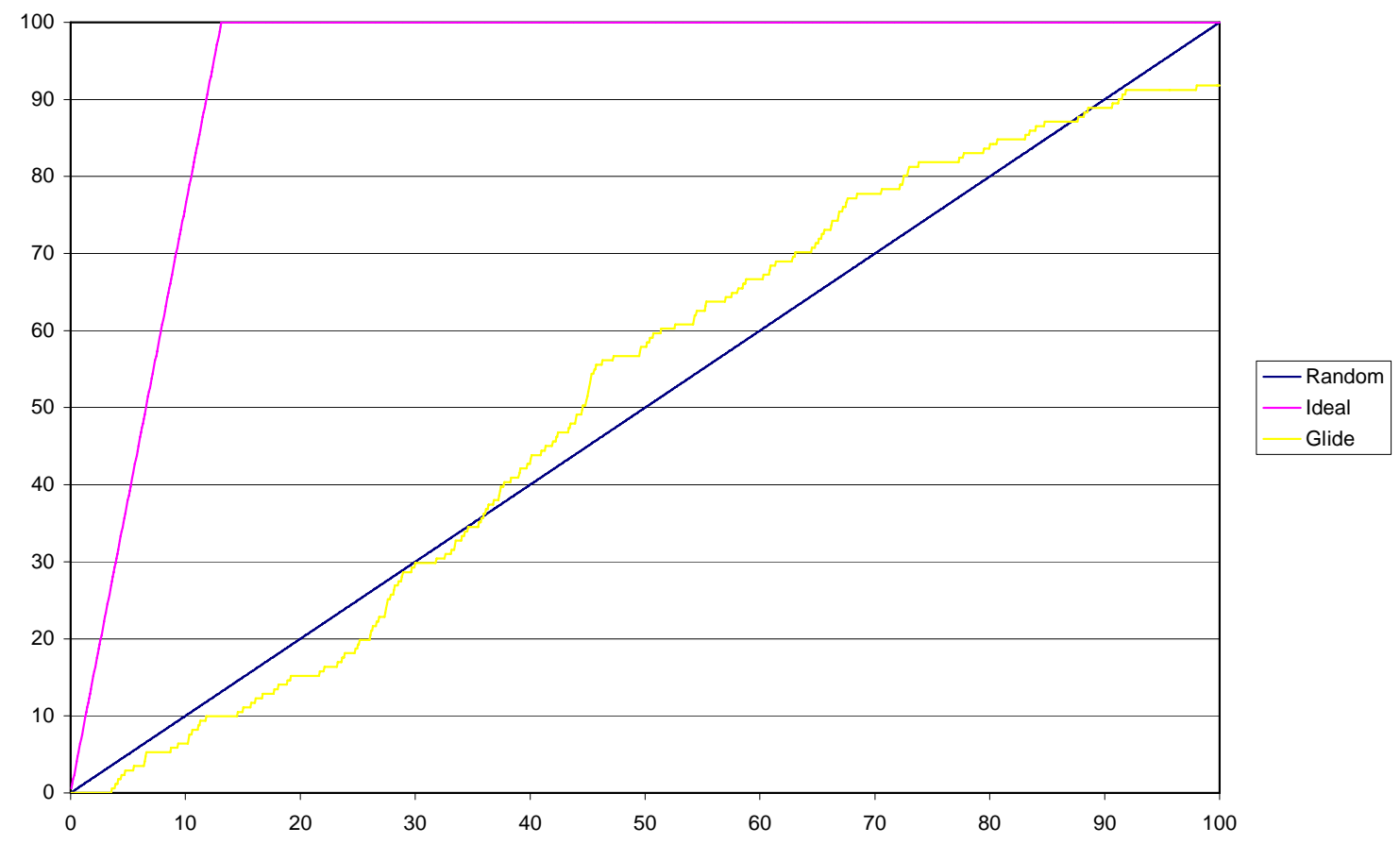

Glide

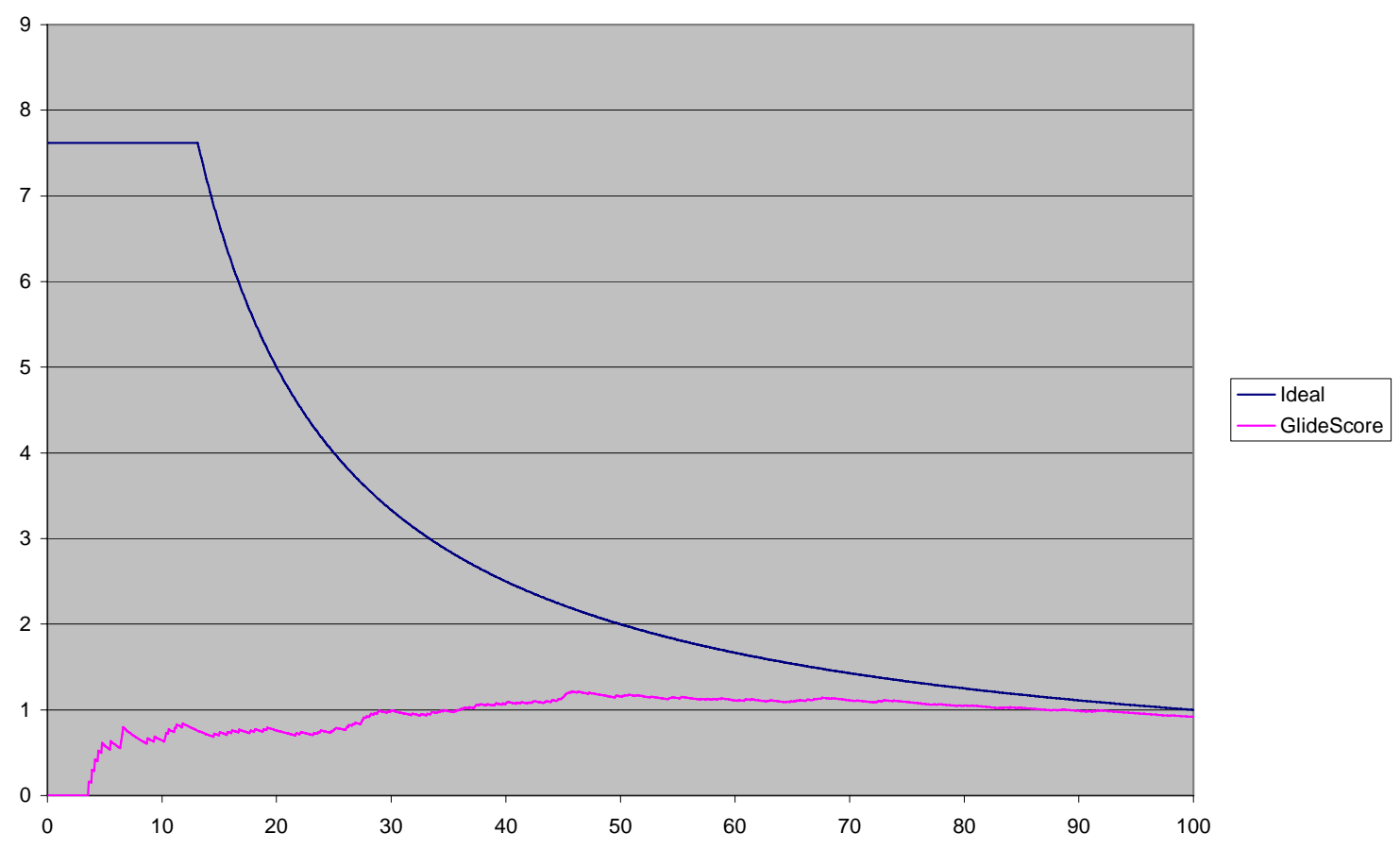


Gold

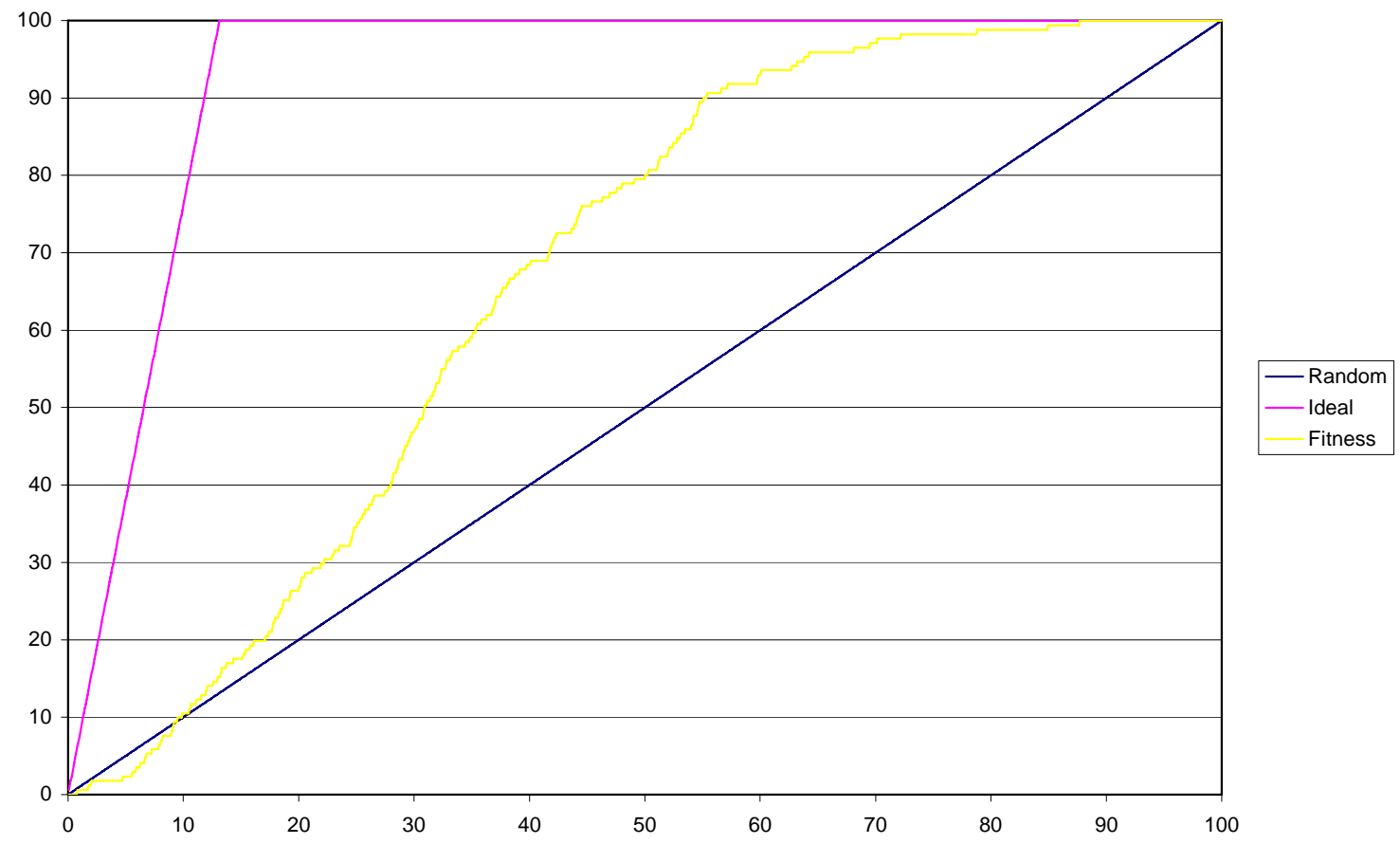

Gold

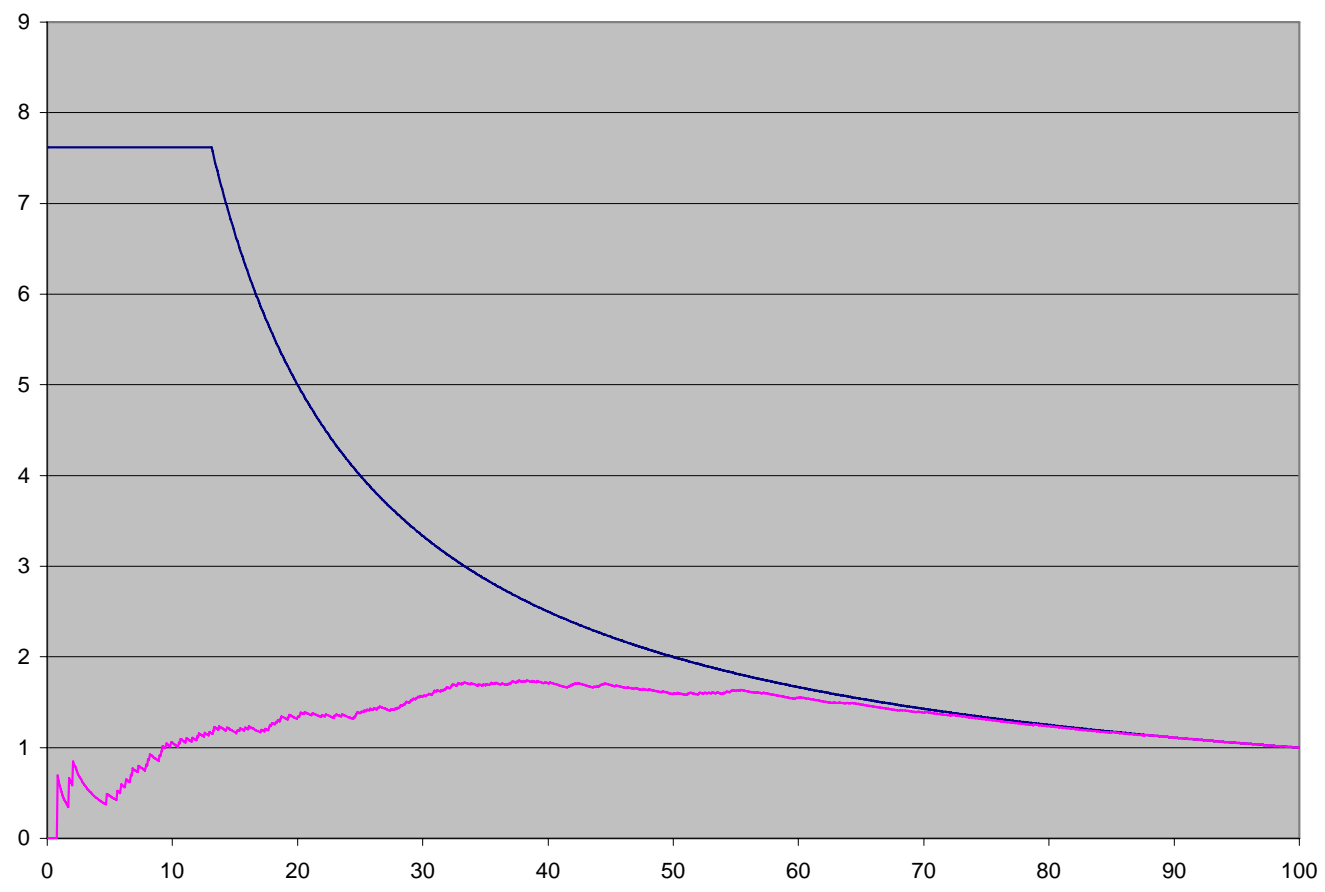


LigFit

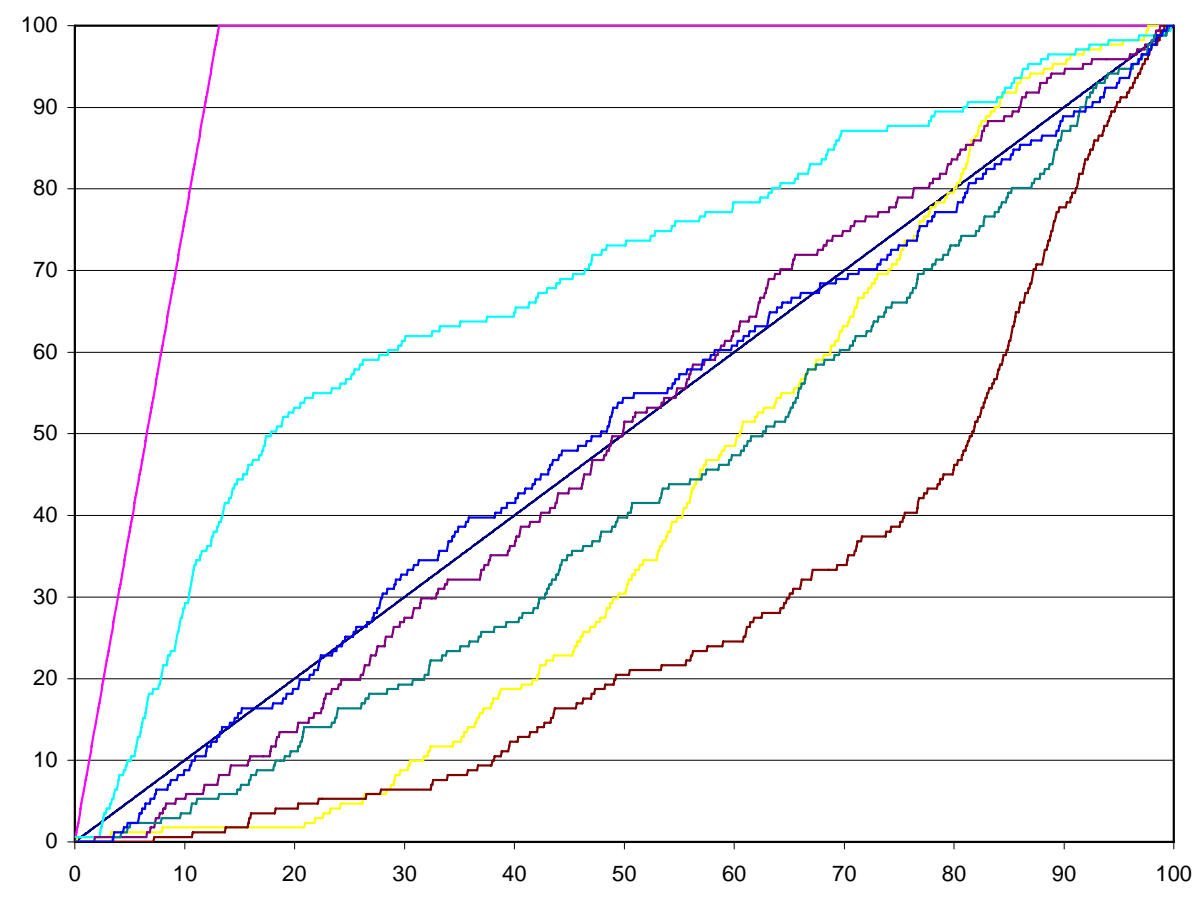

\begin{tabular}{|l|}
\hline - Random \\
- Ideal \\
DrugScore, CFF \\
- DrugScore, Dreiding \\
- Ligscore2, CFF \\
- Ligscore2, Dreiding \\
- PMF, CFF \\
- PMF, Dreiding \\
\hline
\end{tabular}

LigFit

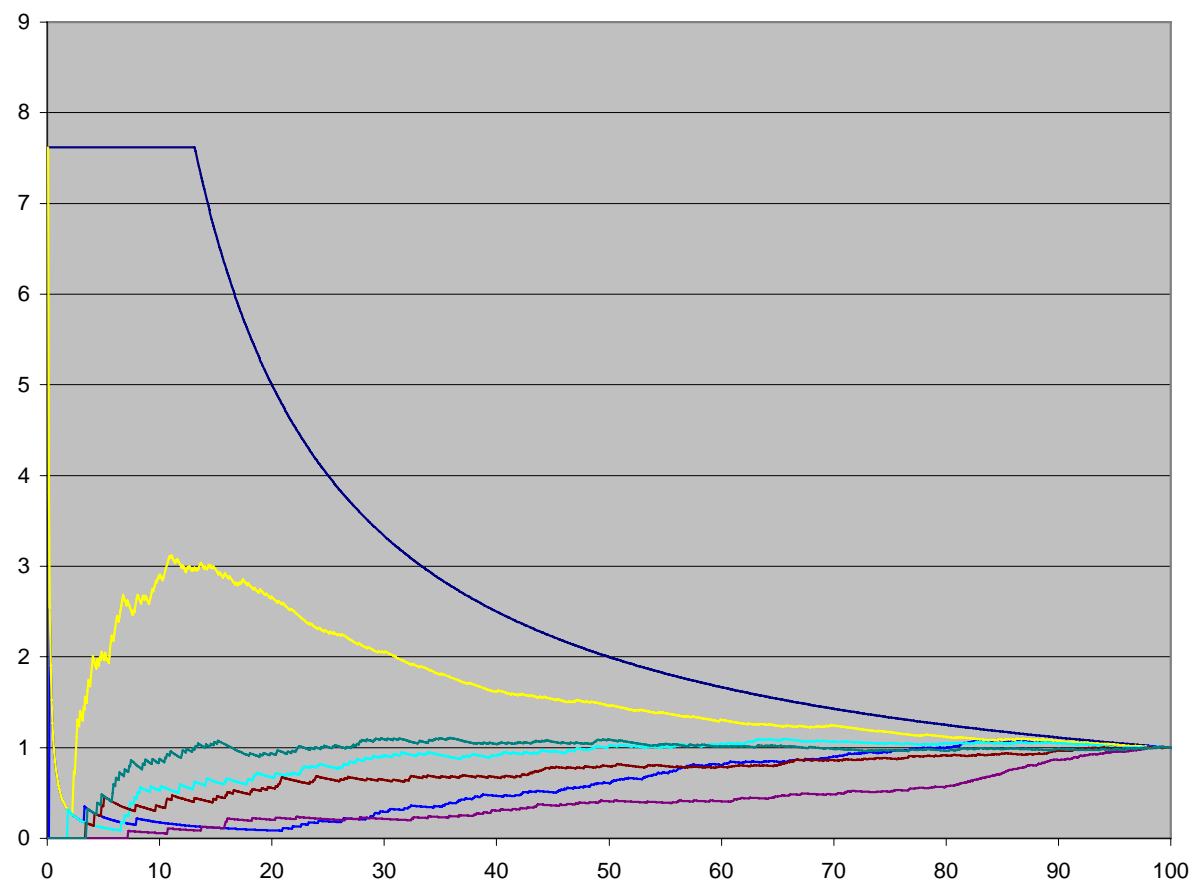

- Ideal
— DrugScore, CFF
DrugScore, Dreiding
- Ligscore2, CFF
- Ligscore2, Dreiding
- PMF, CFF
- PMF, Dreiding


MOE

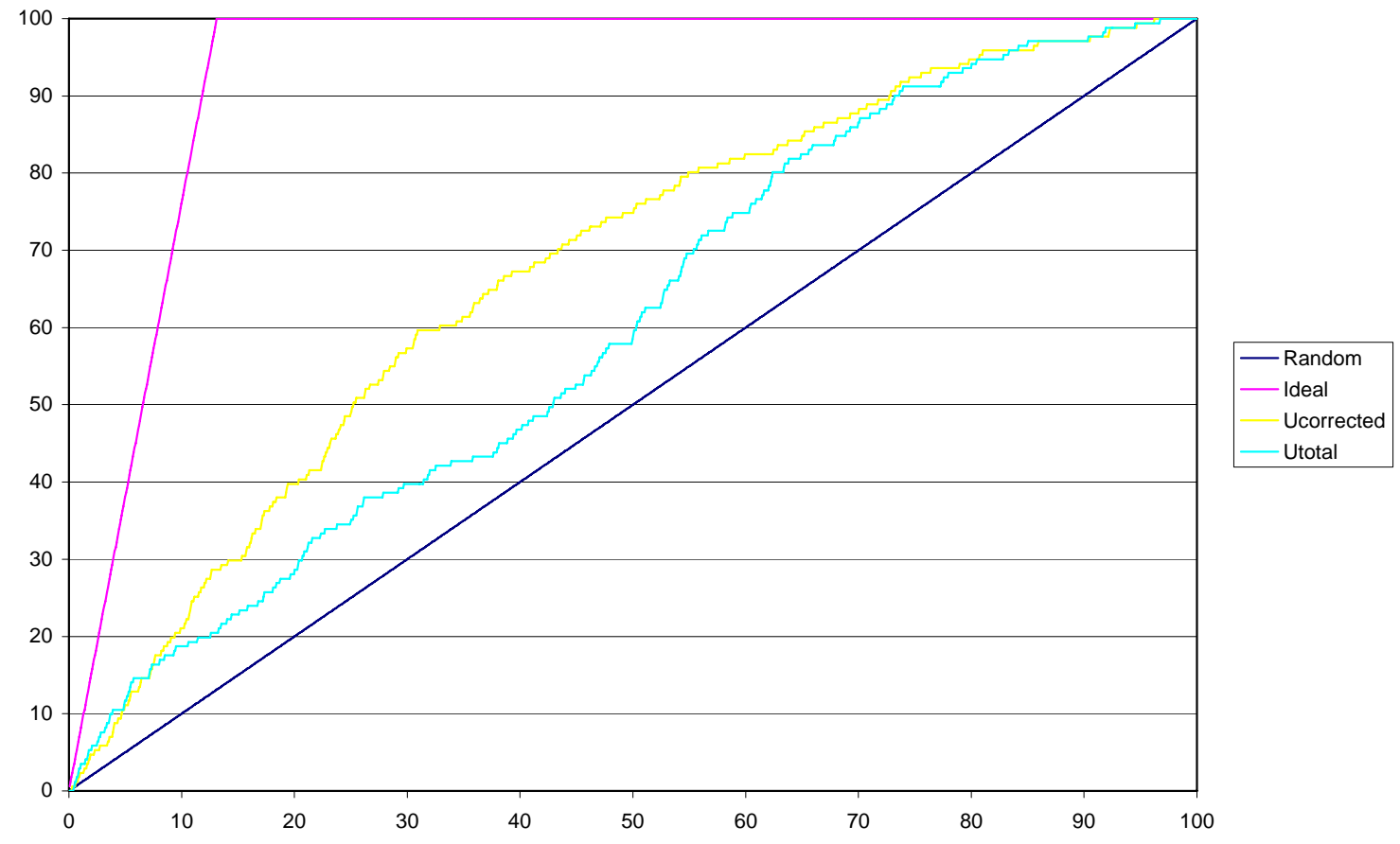

MOE

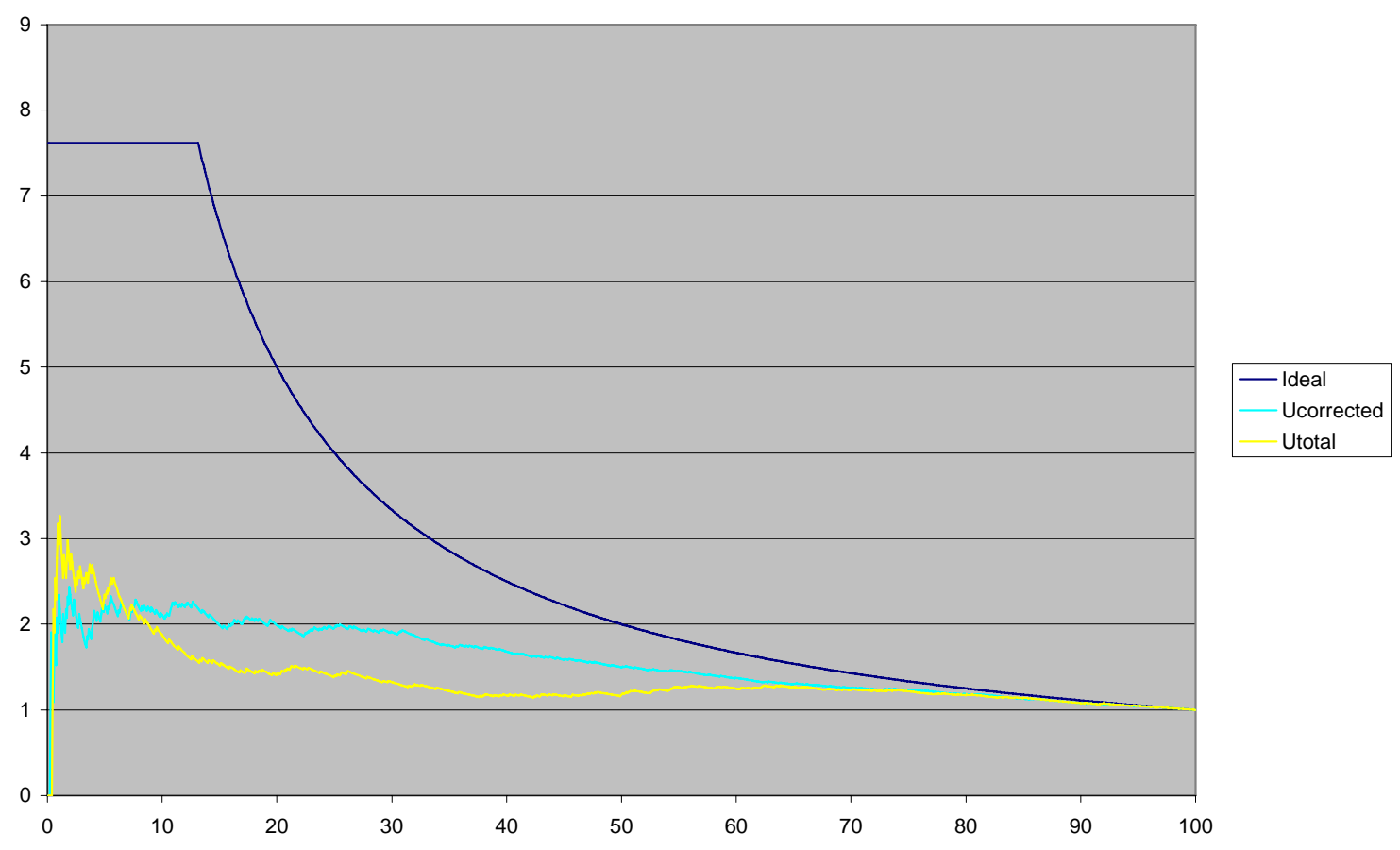




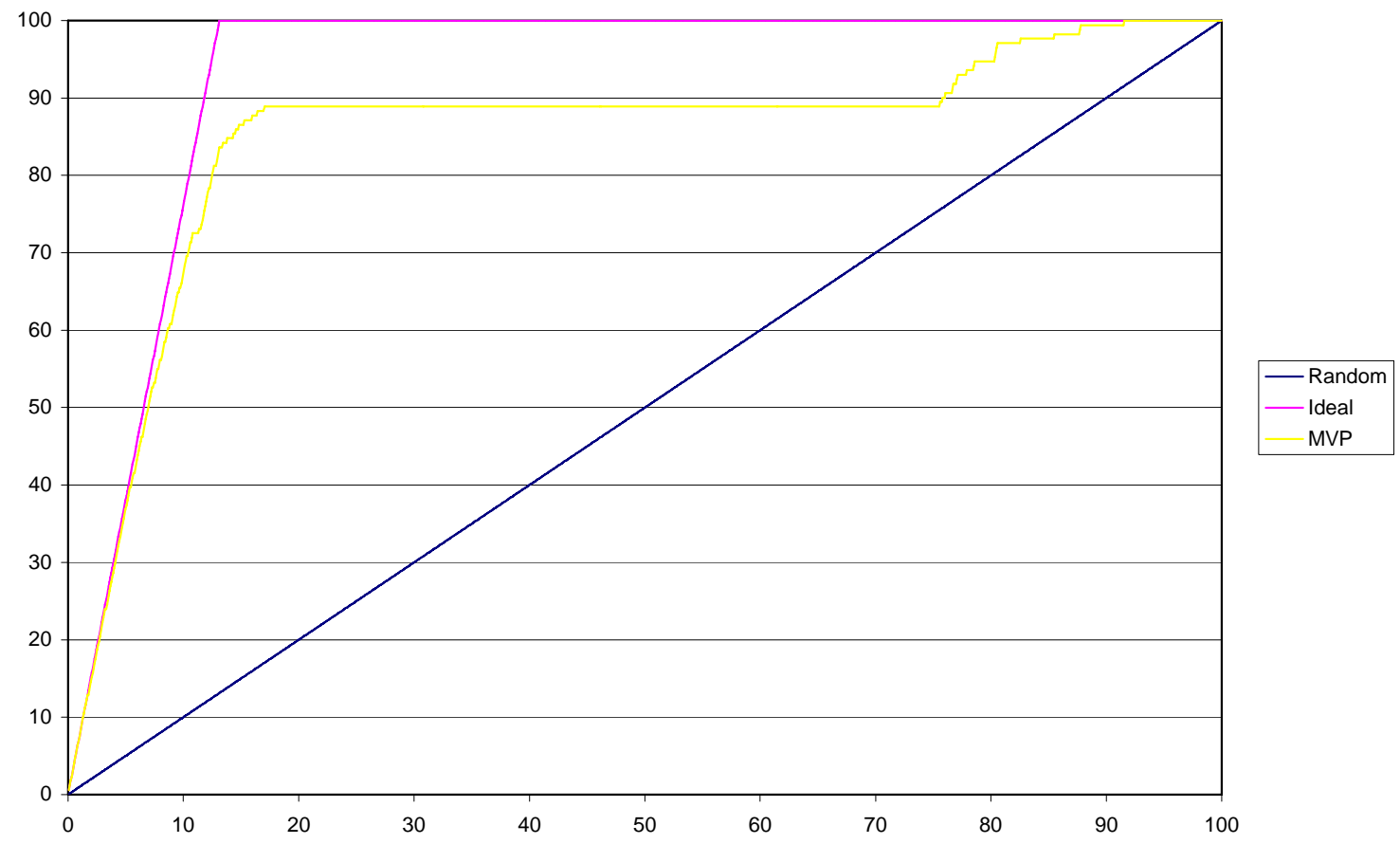

MVP

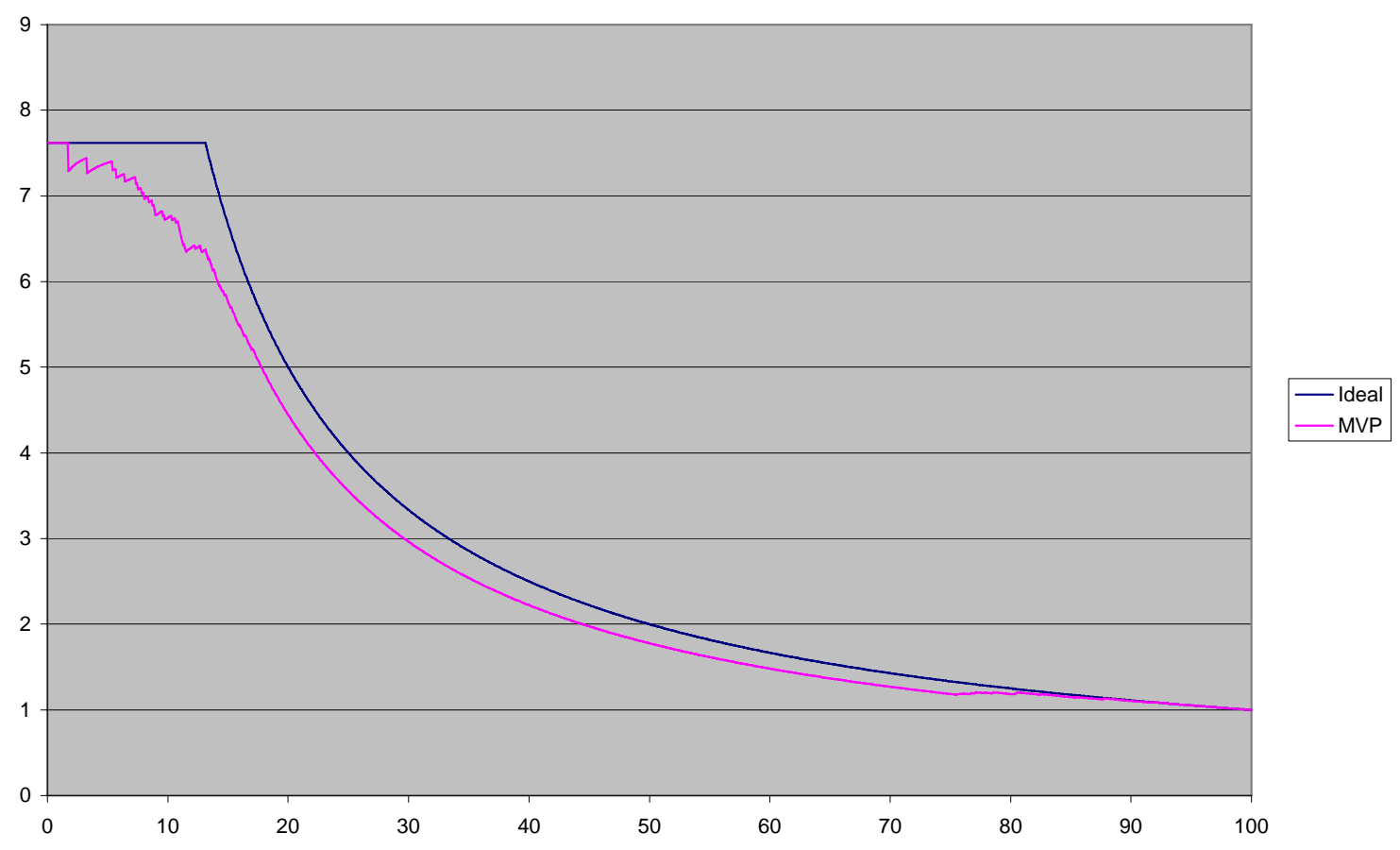


Dock4

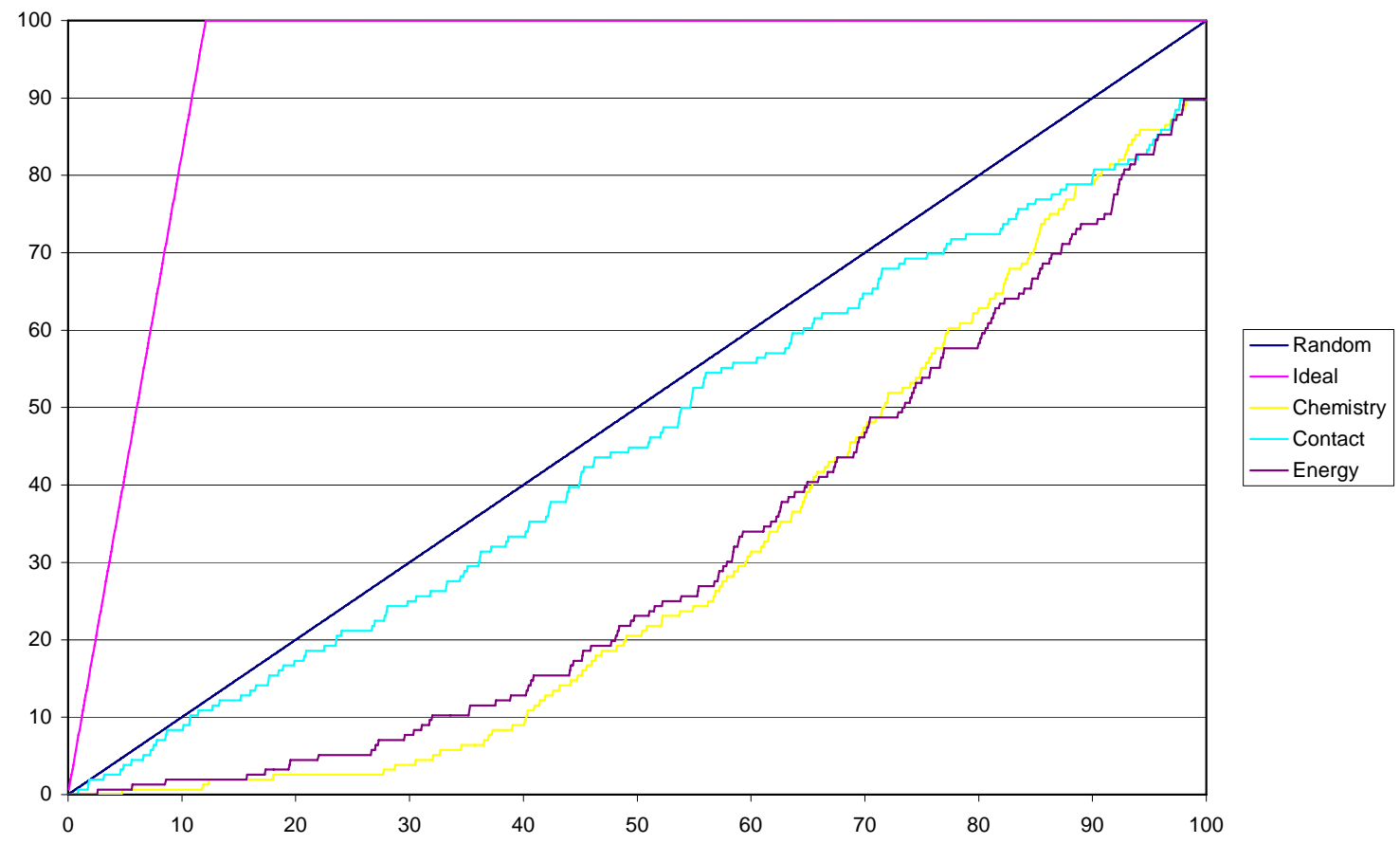

Dock4

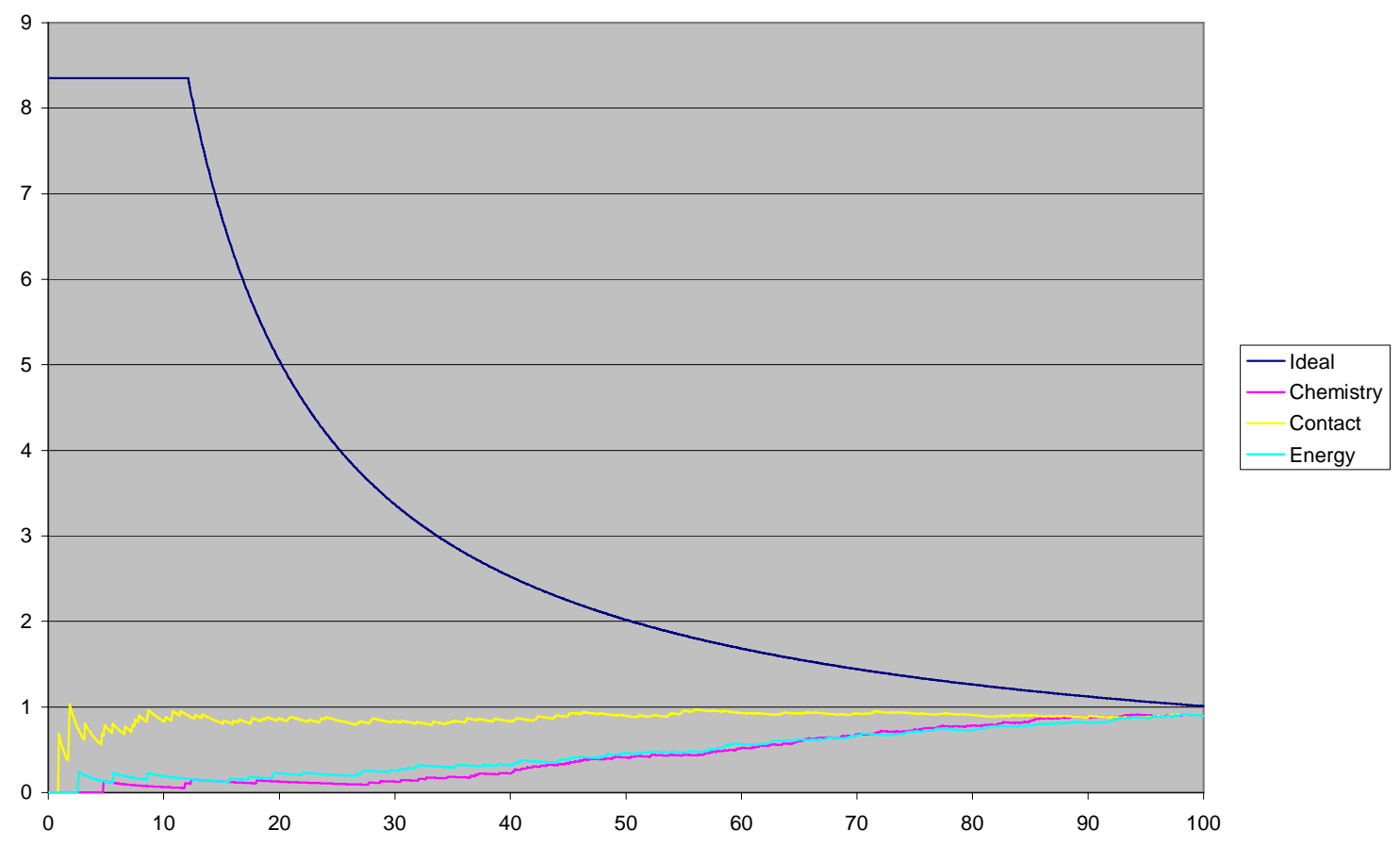




\section{Dockit}

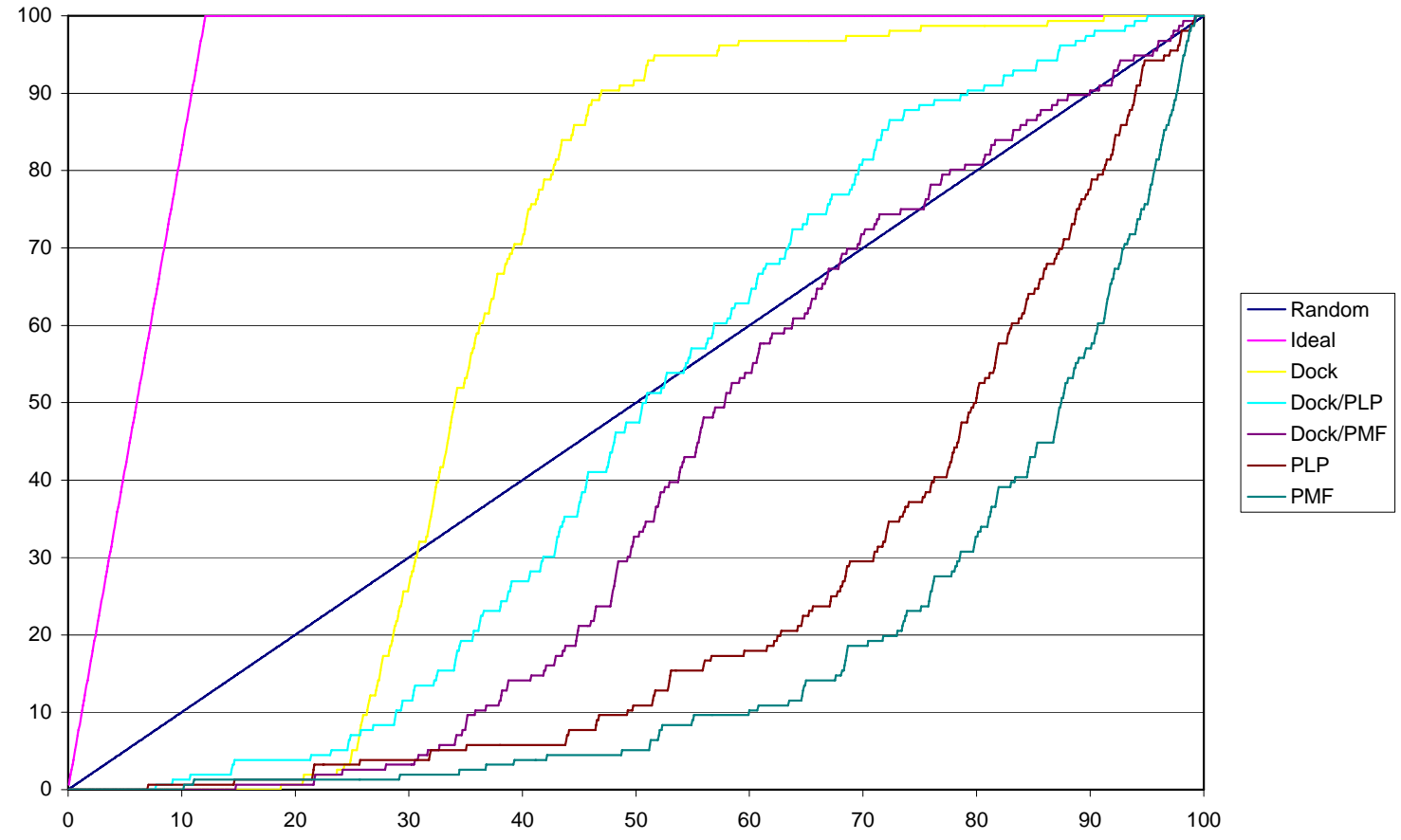

Dockit

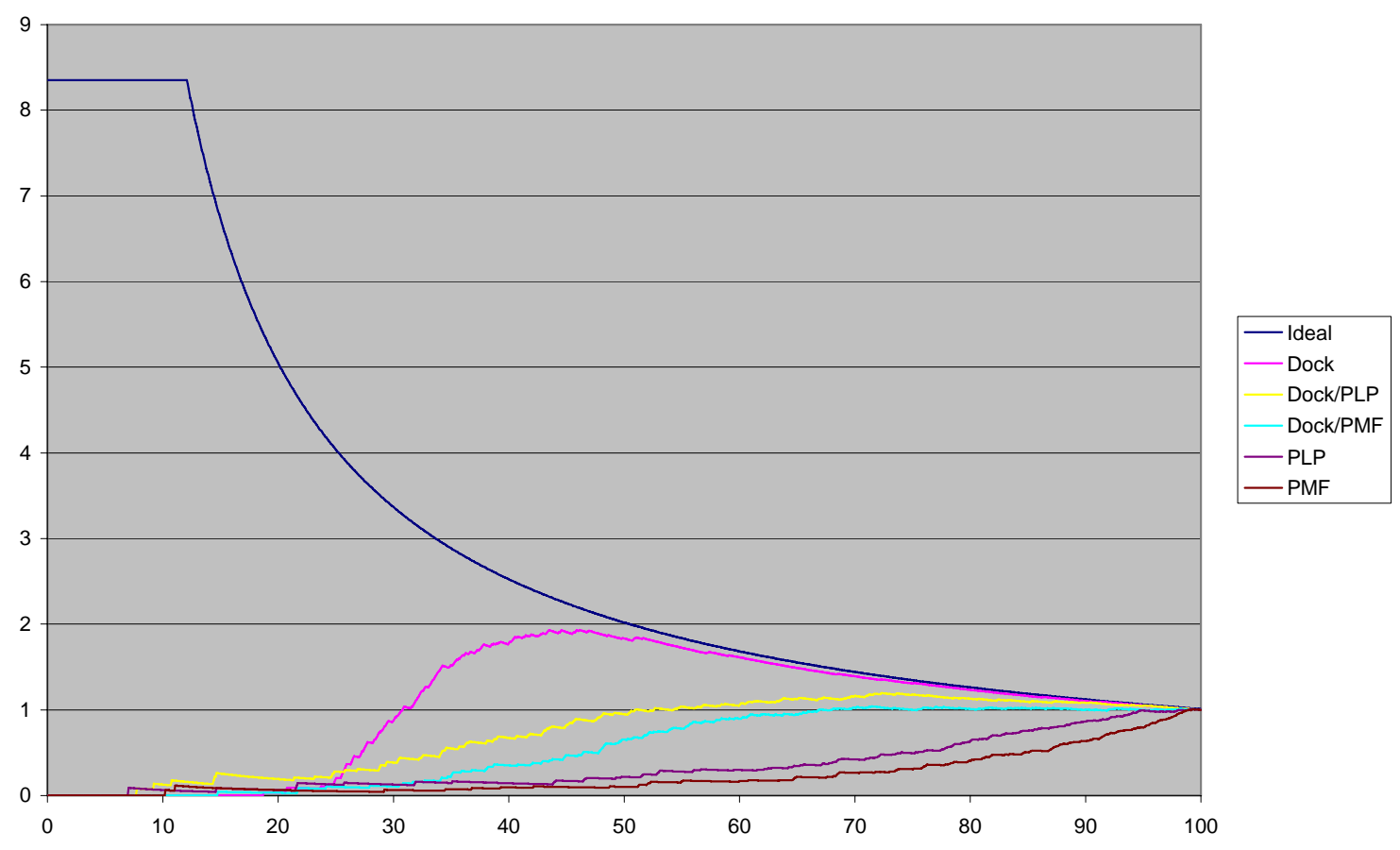


FlexX

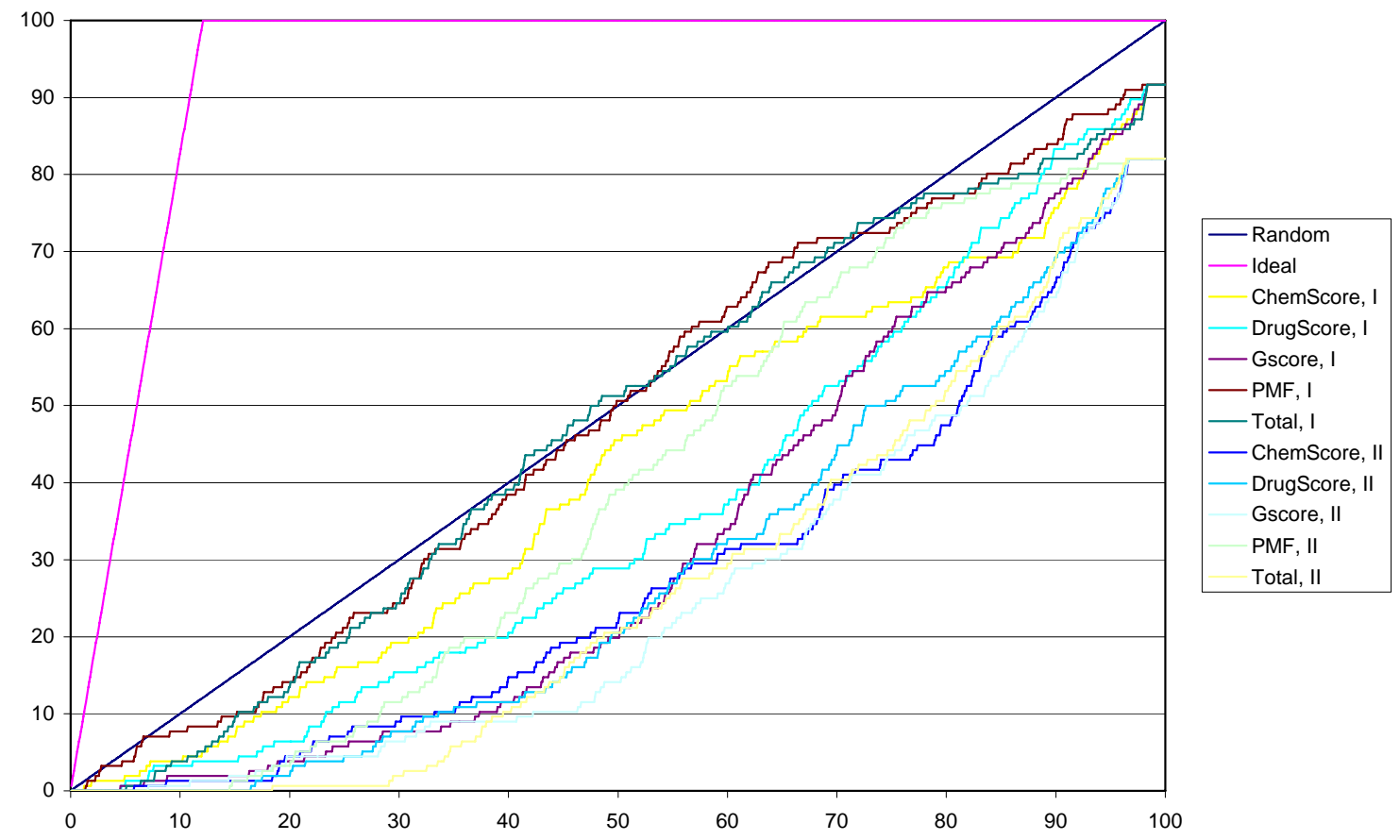

FlexX

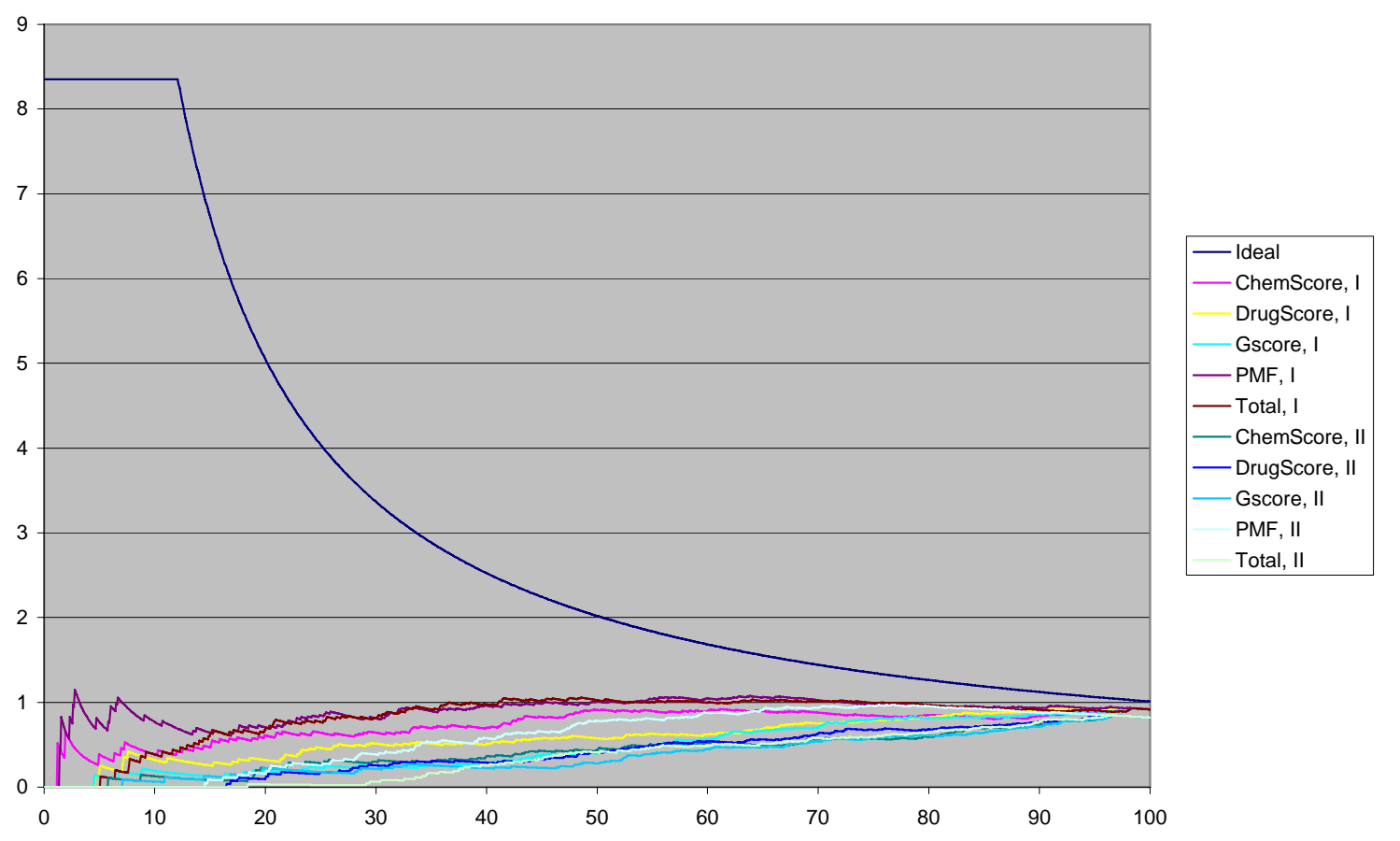




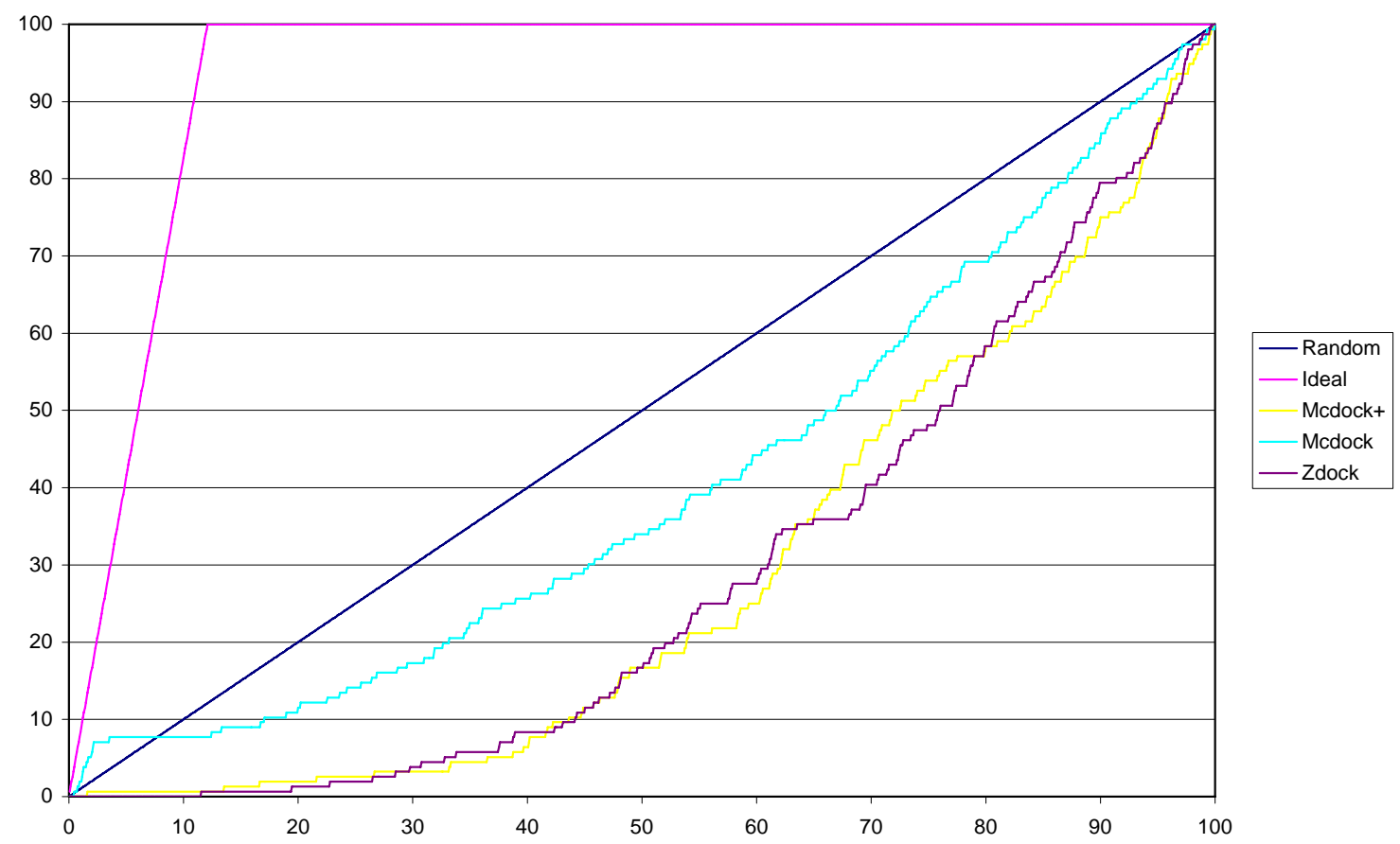

Flo

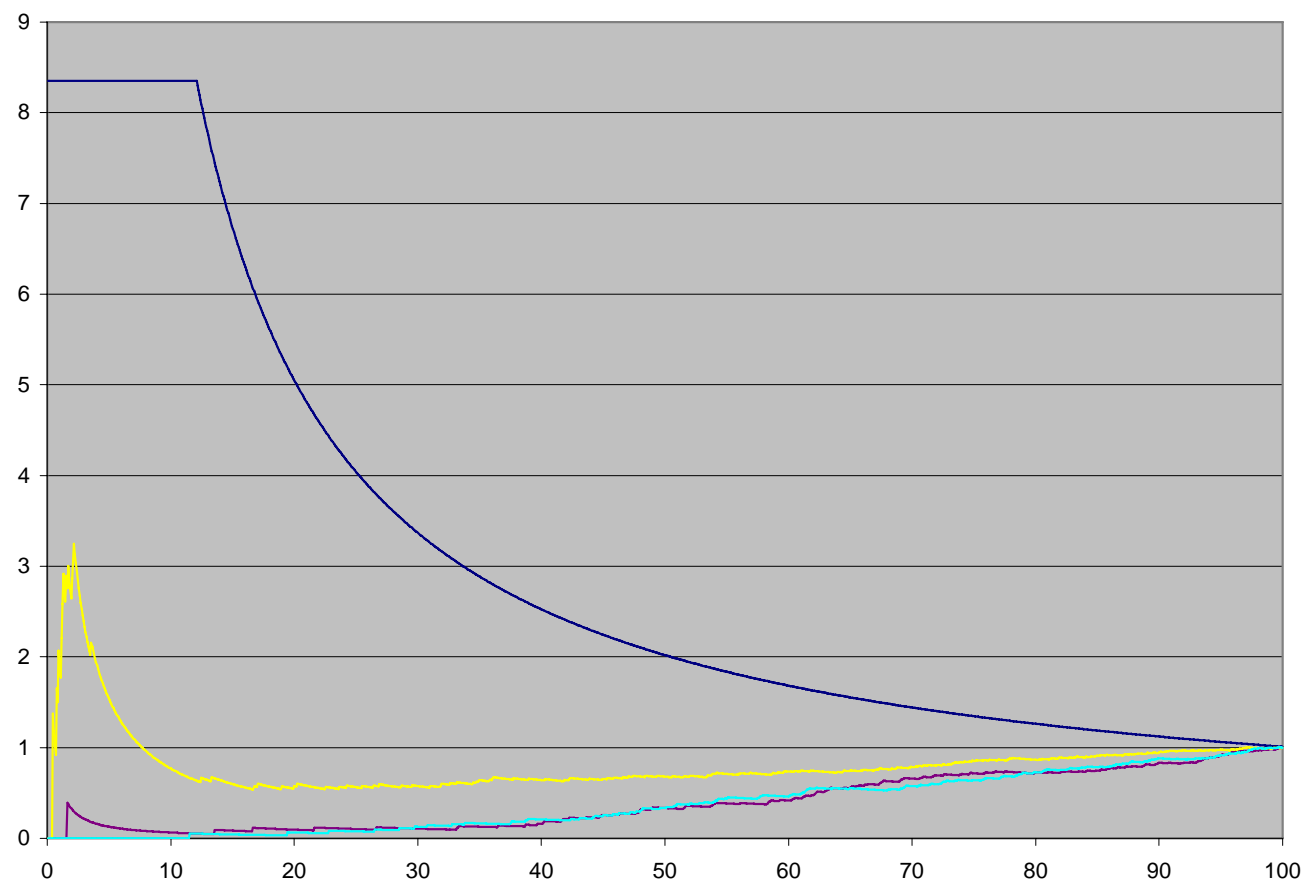


Fred
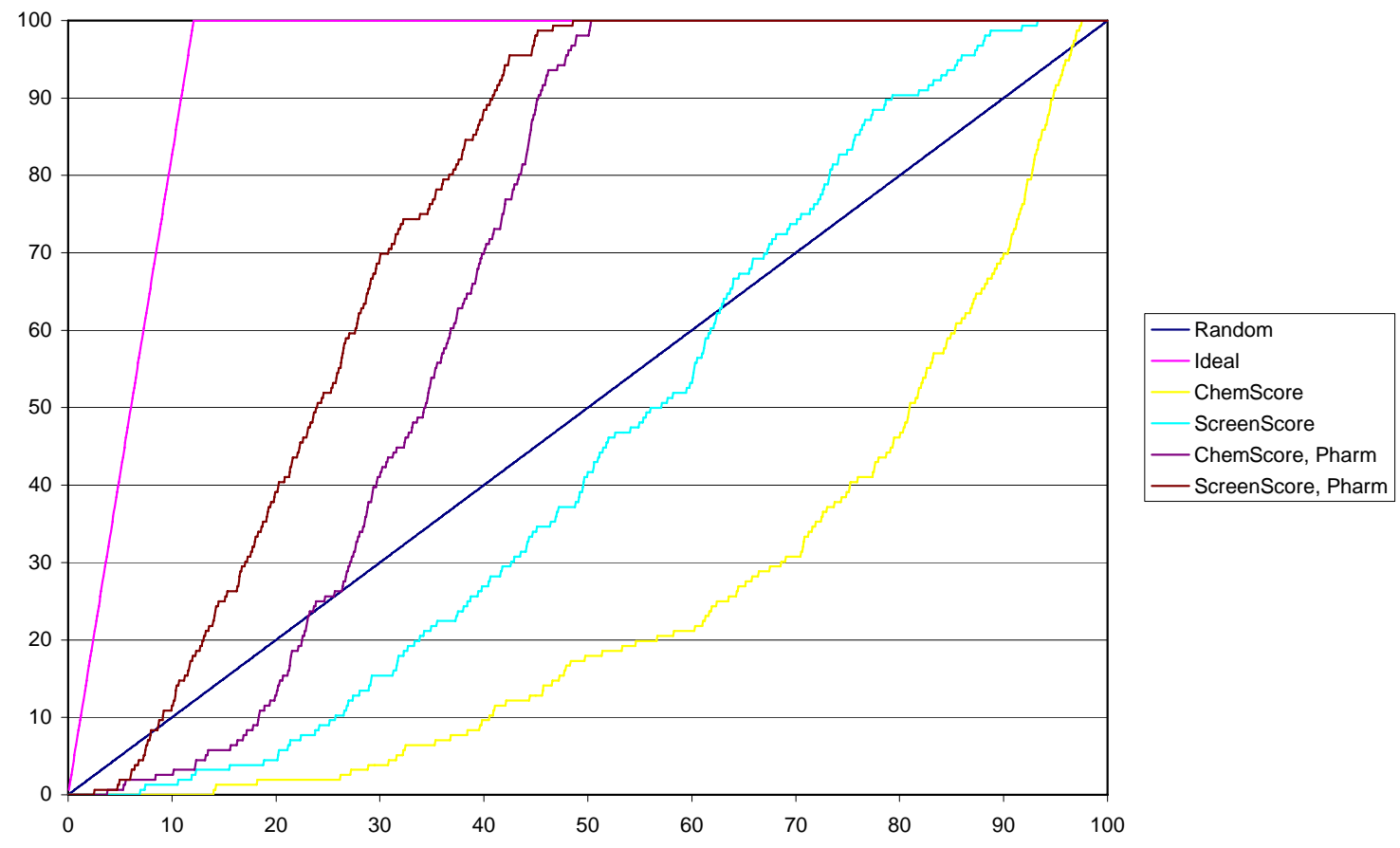

Fred

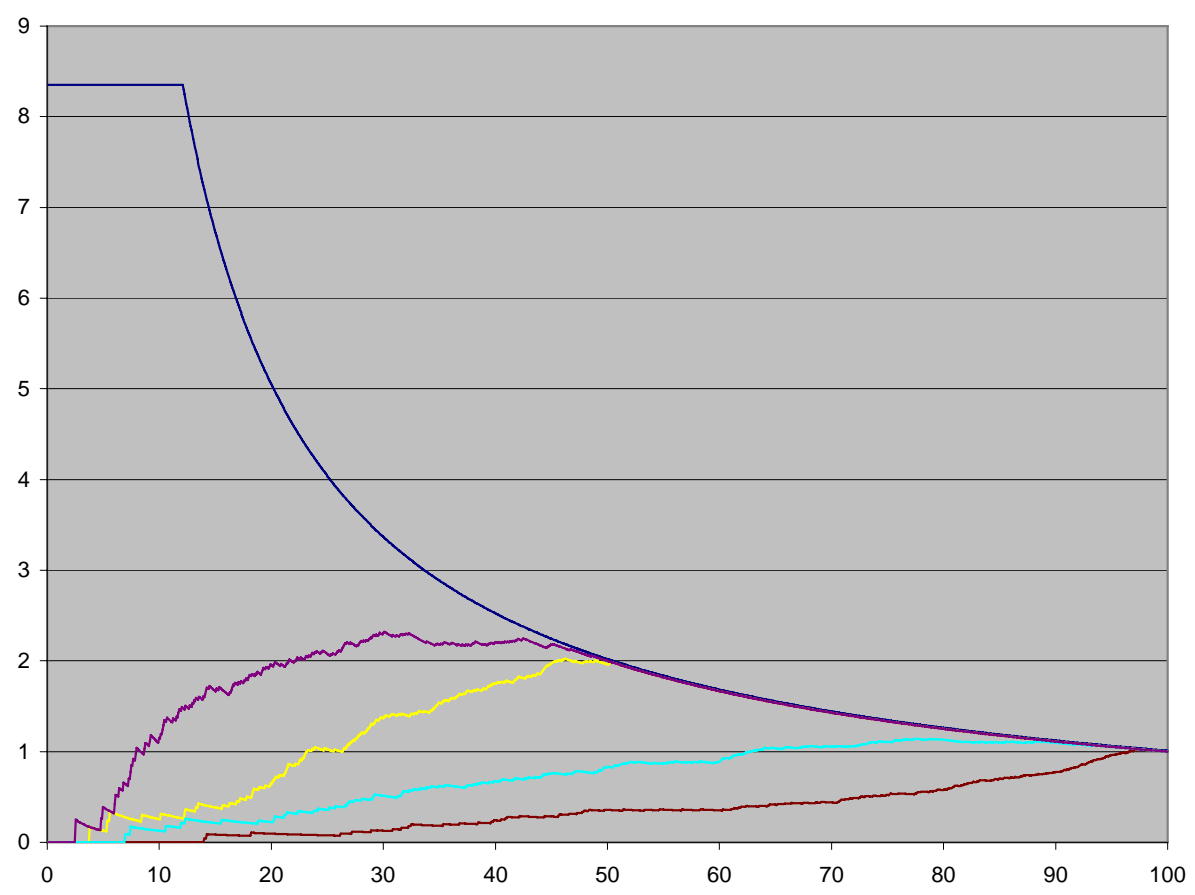

\begin{tabular}{|l|}
\hline - Ideal \\
- ChemScore \\
ChemScore, Pharm \\
- ScreenScore \\
- ScreenScore, Pharm
\end{tabular}




\section{Glide}

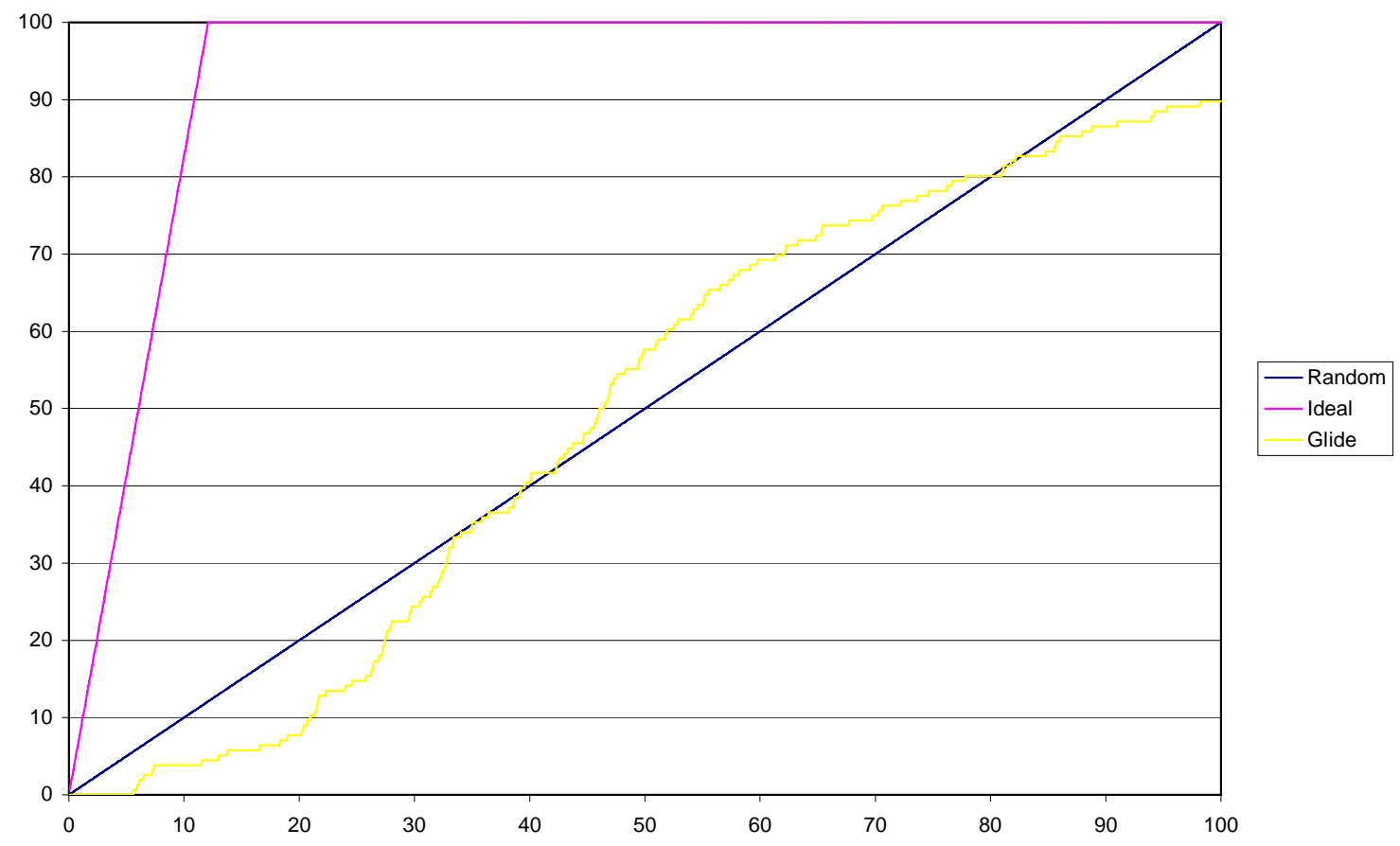

Glide

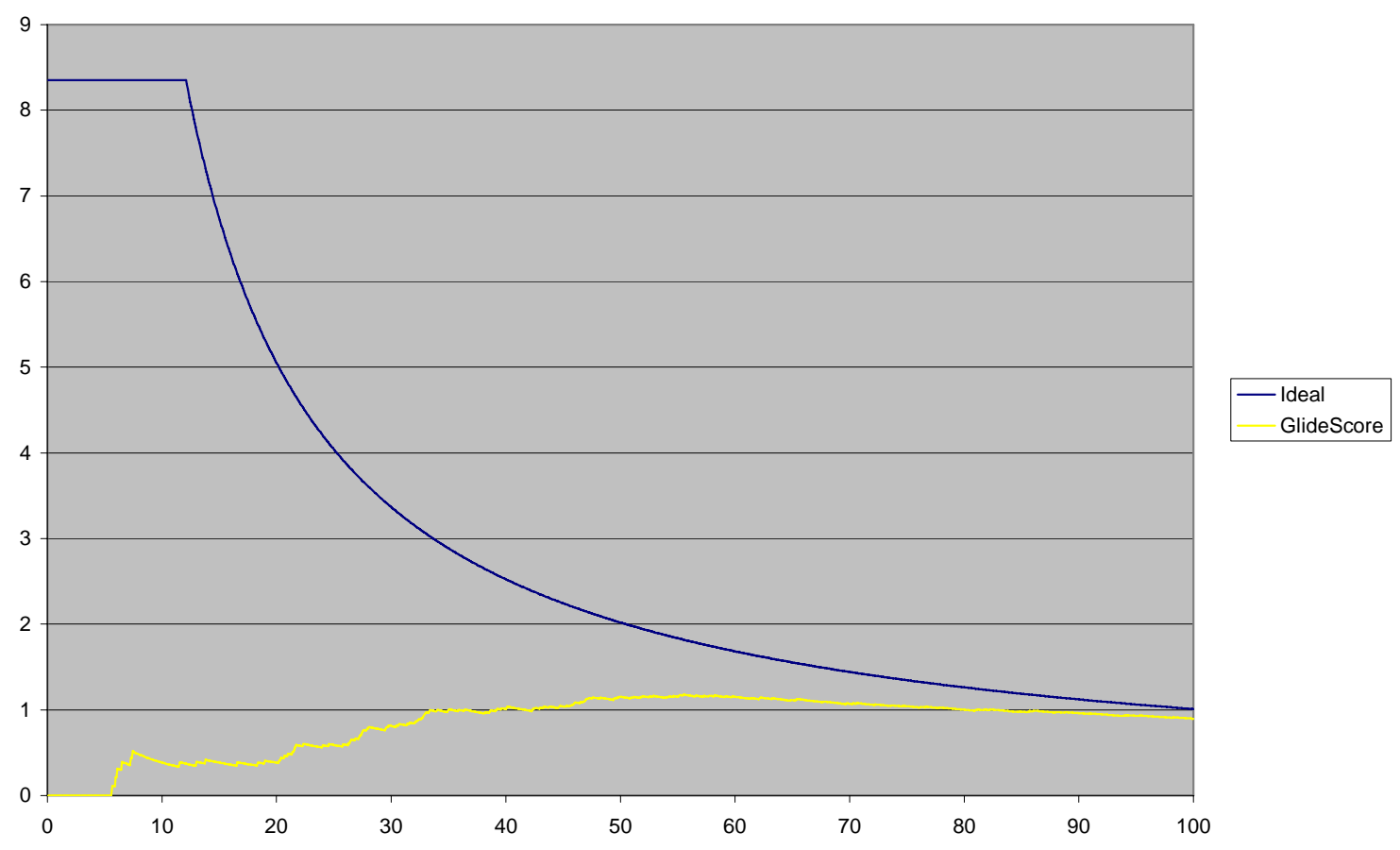


Gold

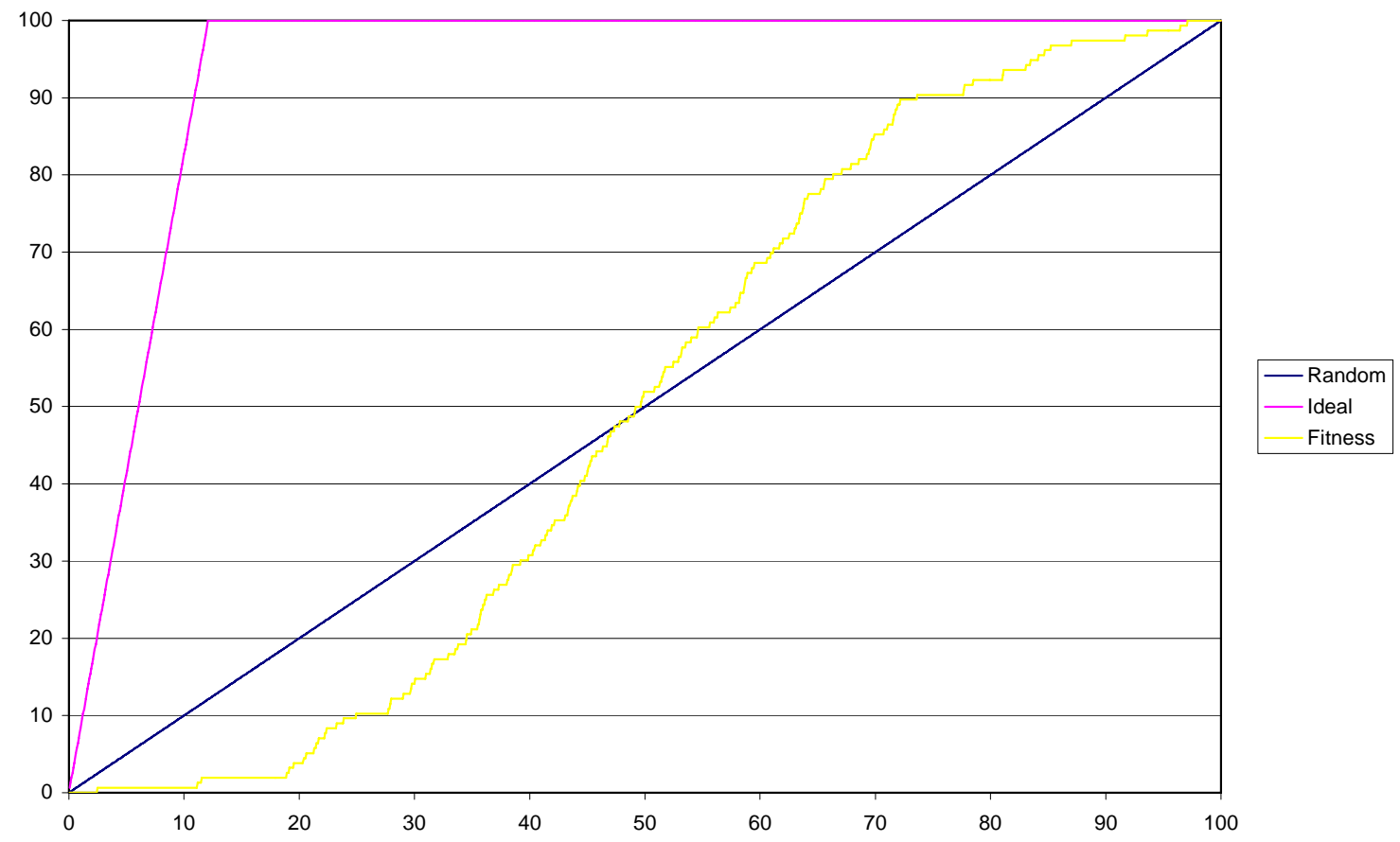

Gold

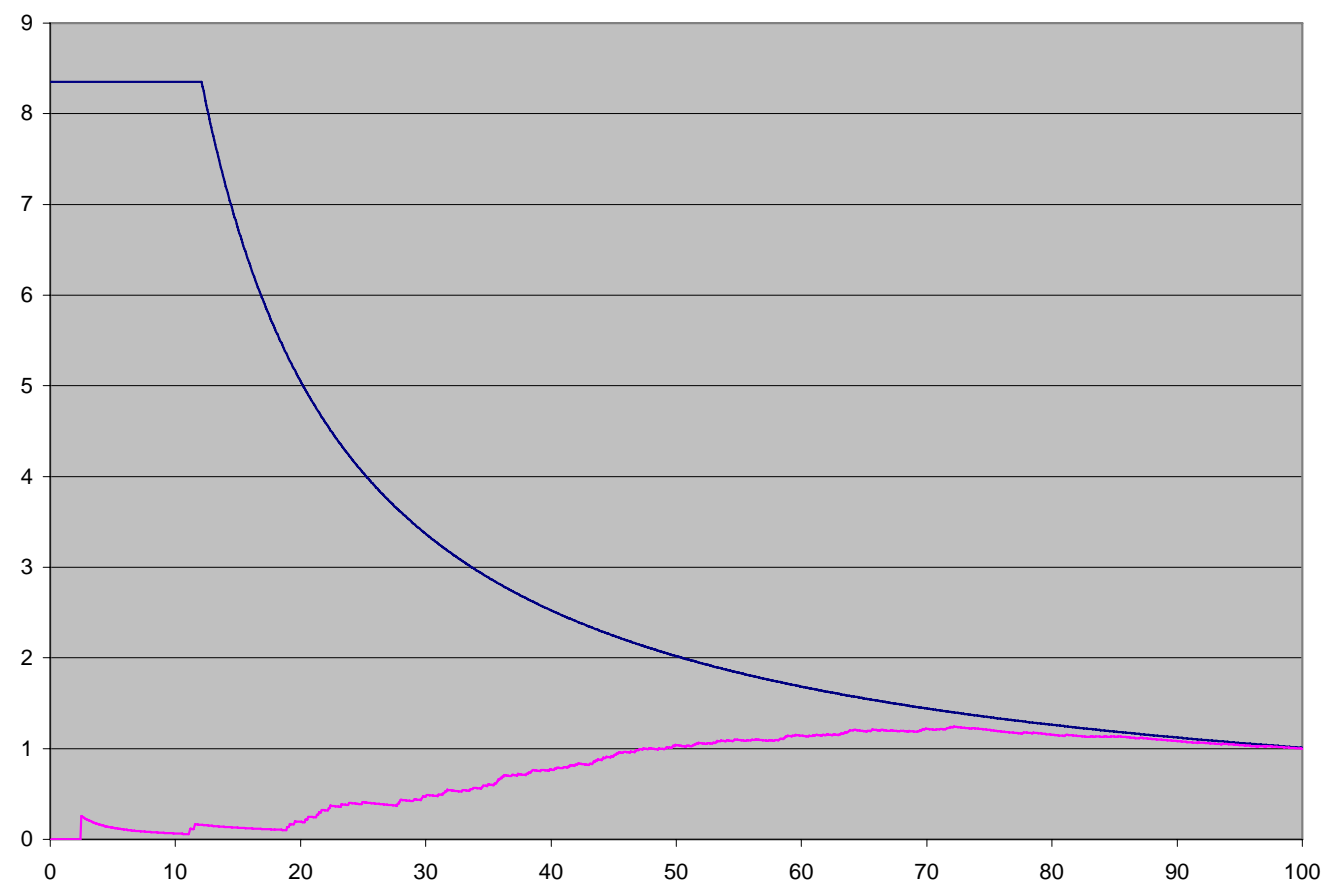




\section{LigFit}

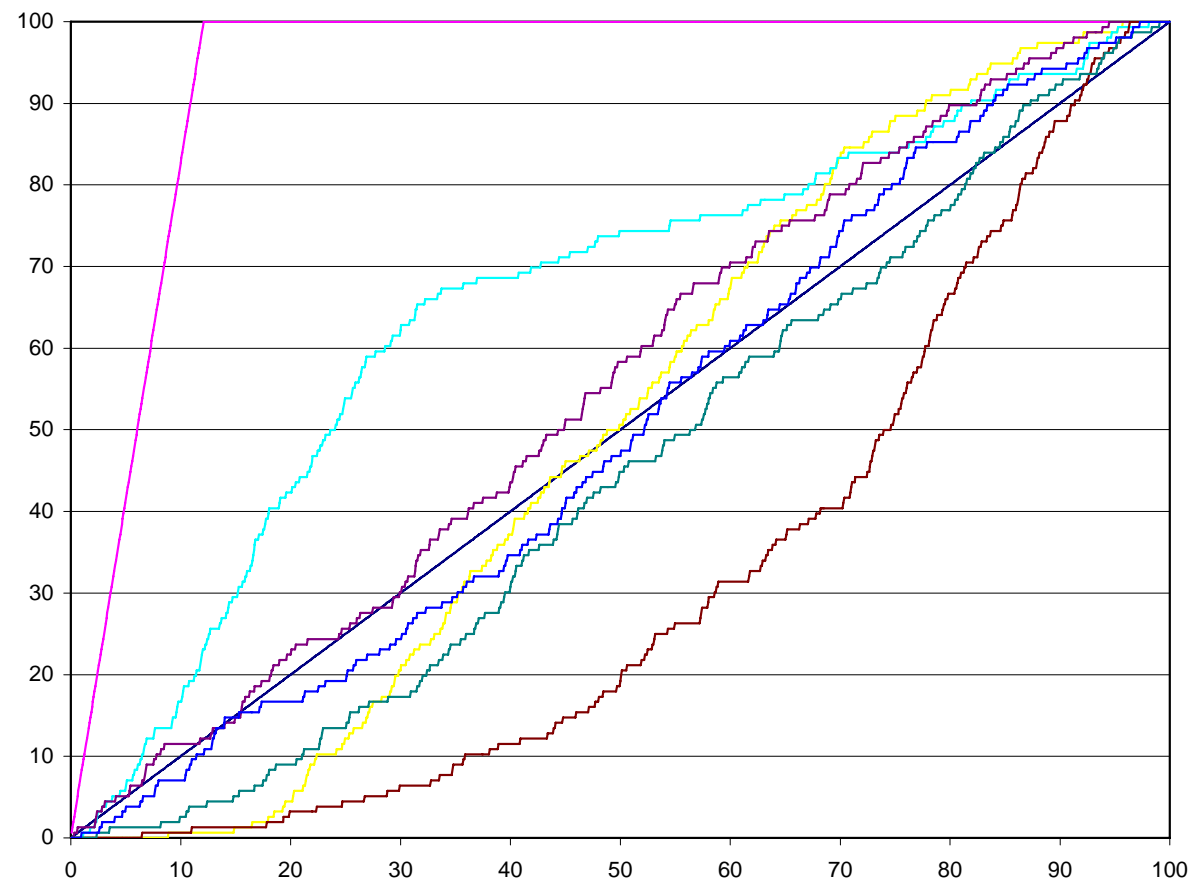

\begin{tabular}{l}
\hline - Random \\
Ideal \\
DrugScore, CFF \\
DrugScore, Dreiding \\
Ligscore2, CFF \\
Ligscore2, Dreiding \\
- PMF, CFF \\
PMF, Dreiding
\end{tabular}

LigFit

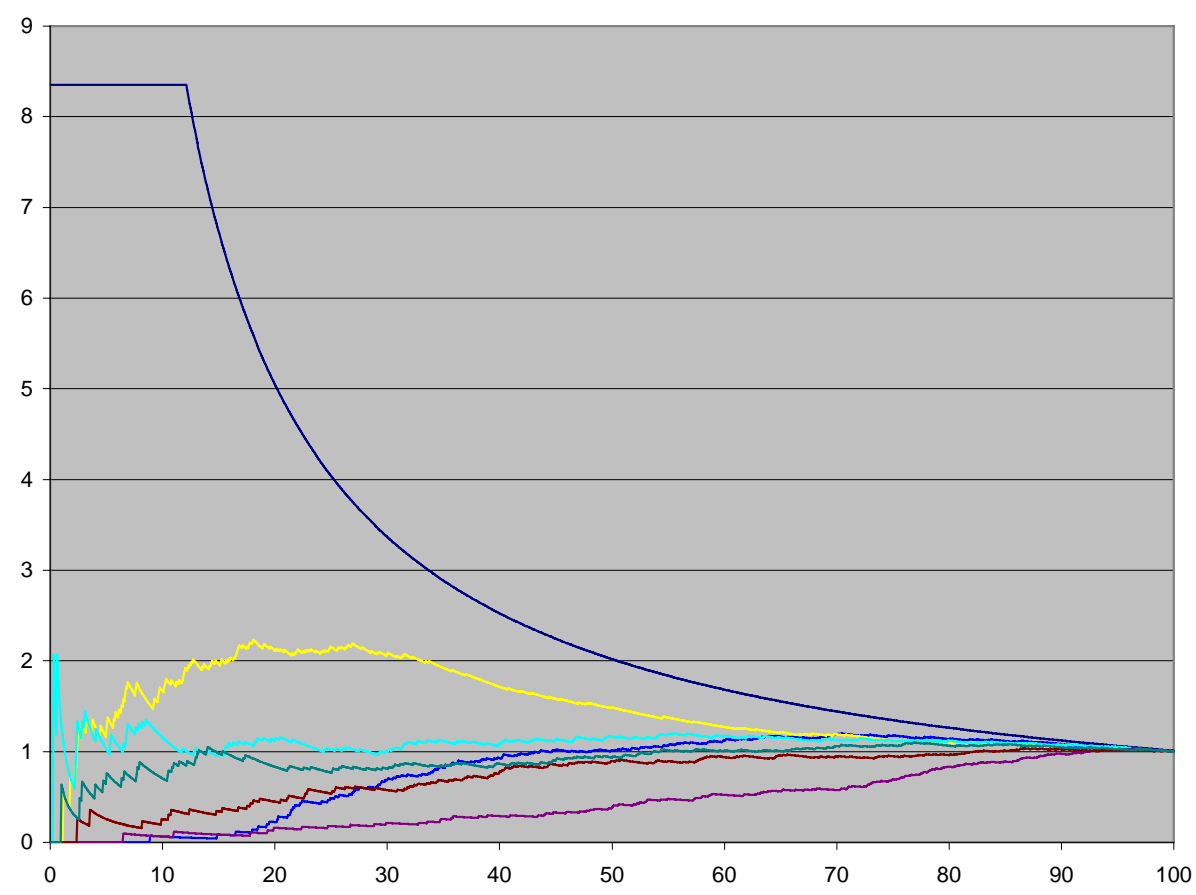


MOE

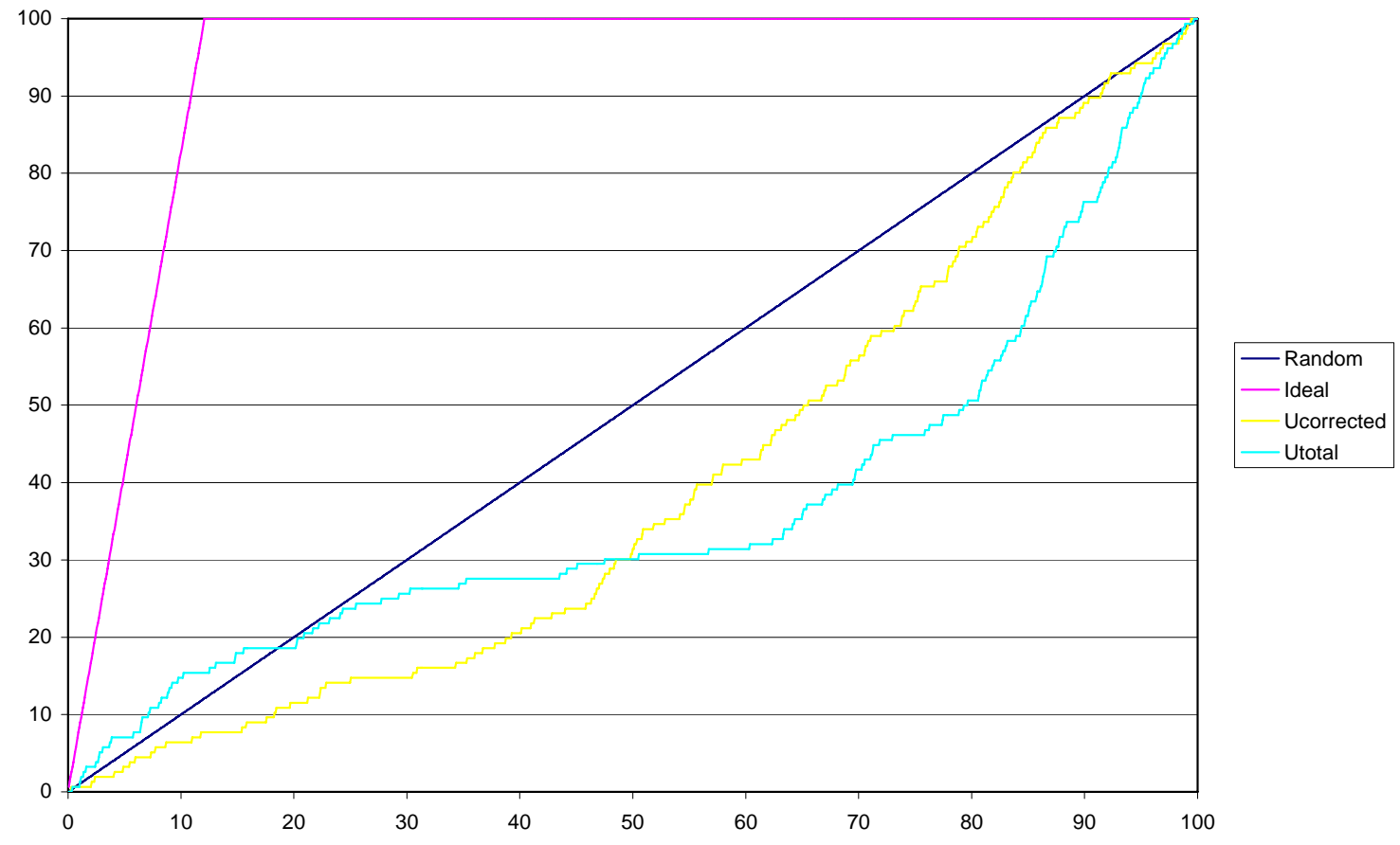

MOE

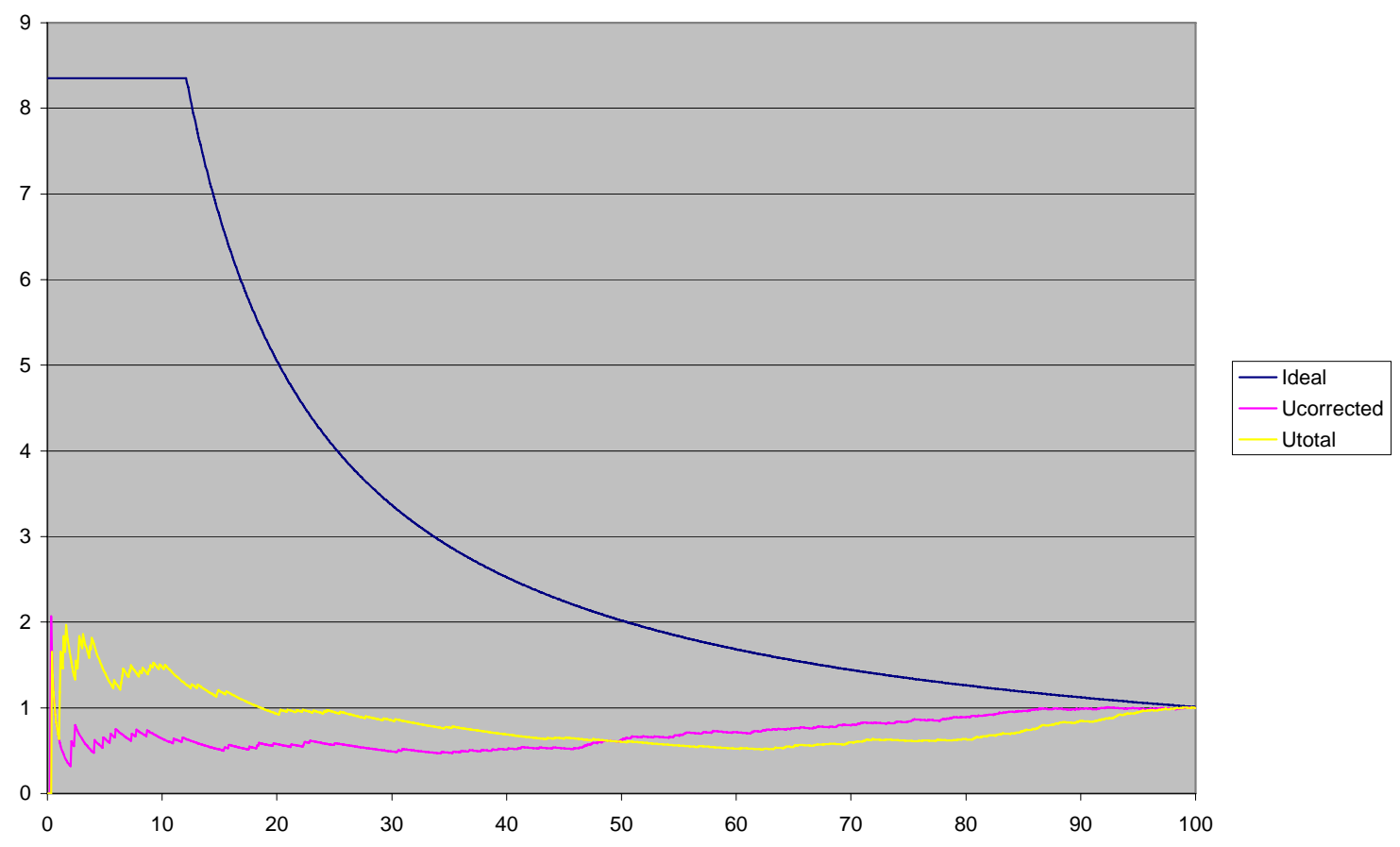


MVP

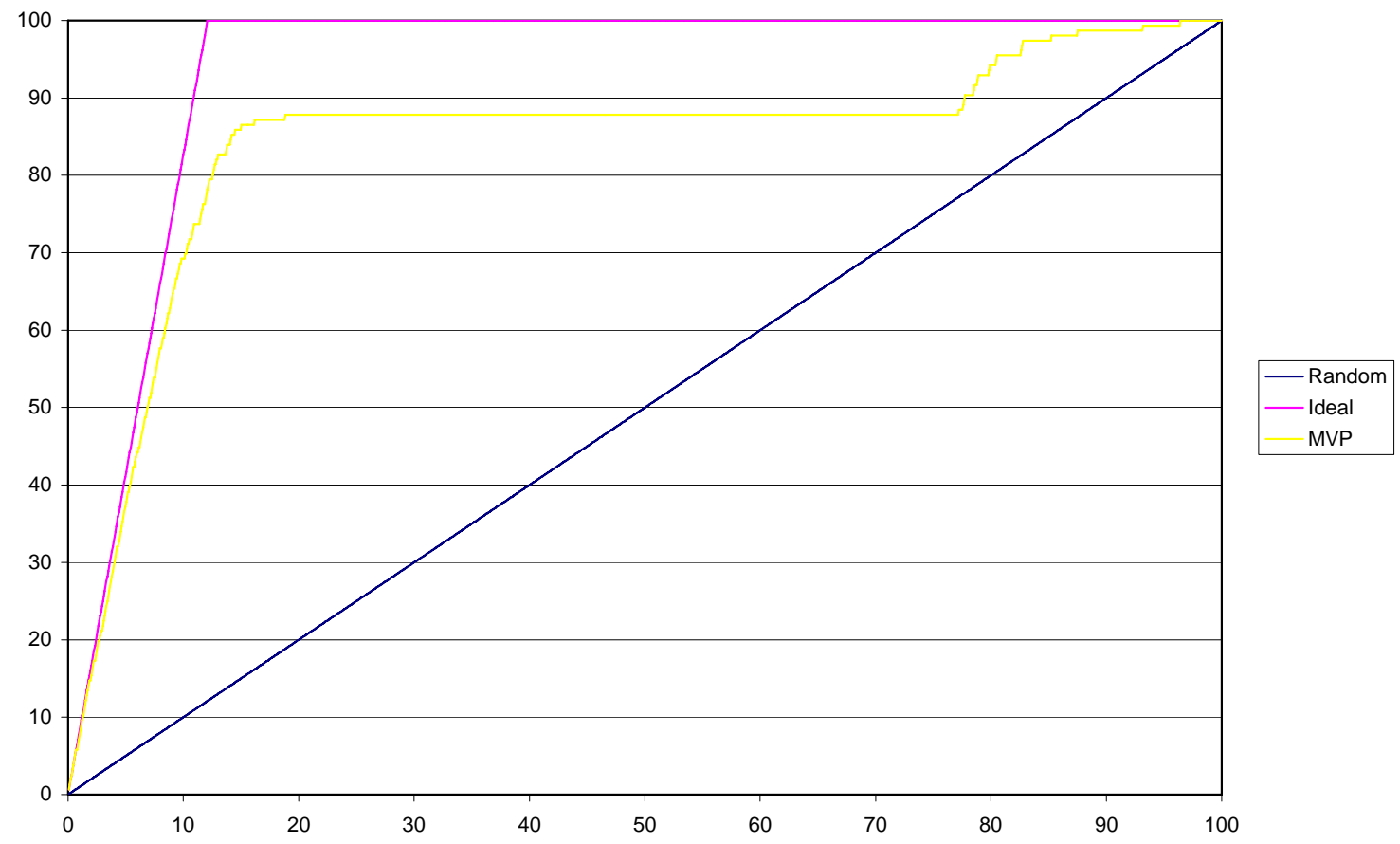

MVP

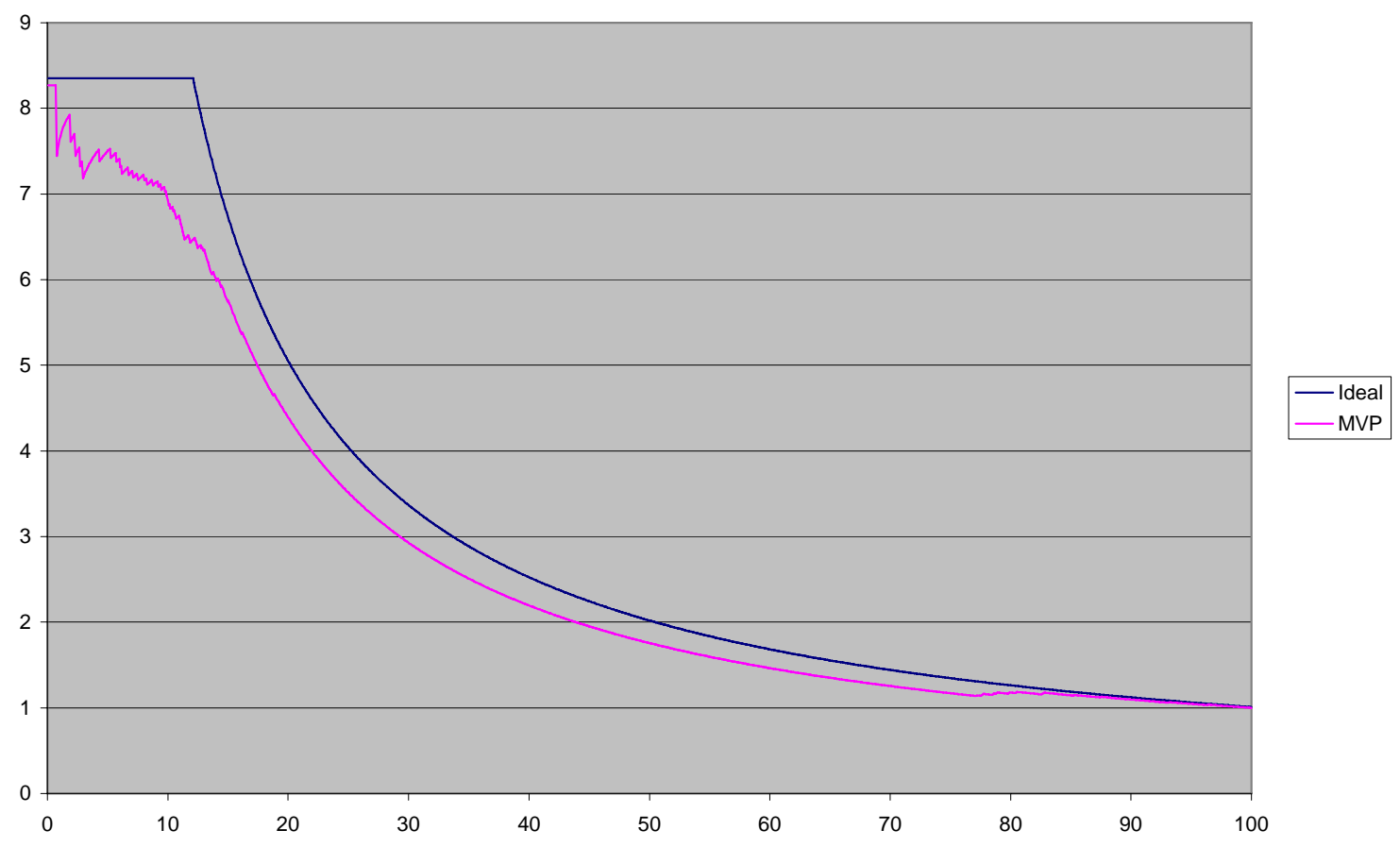


Dock4

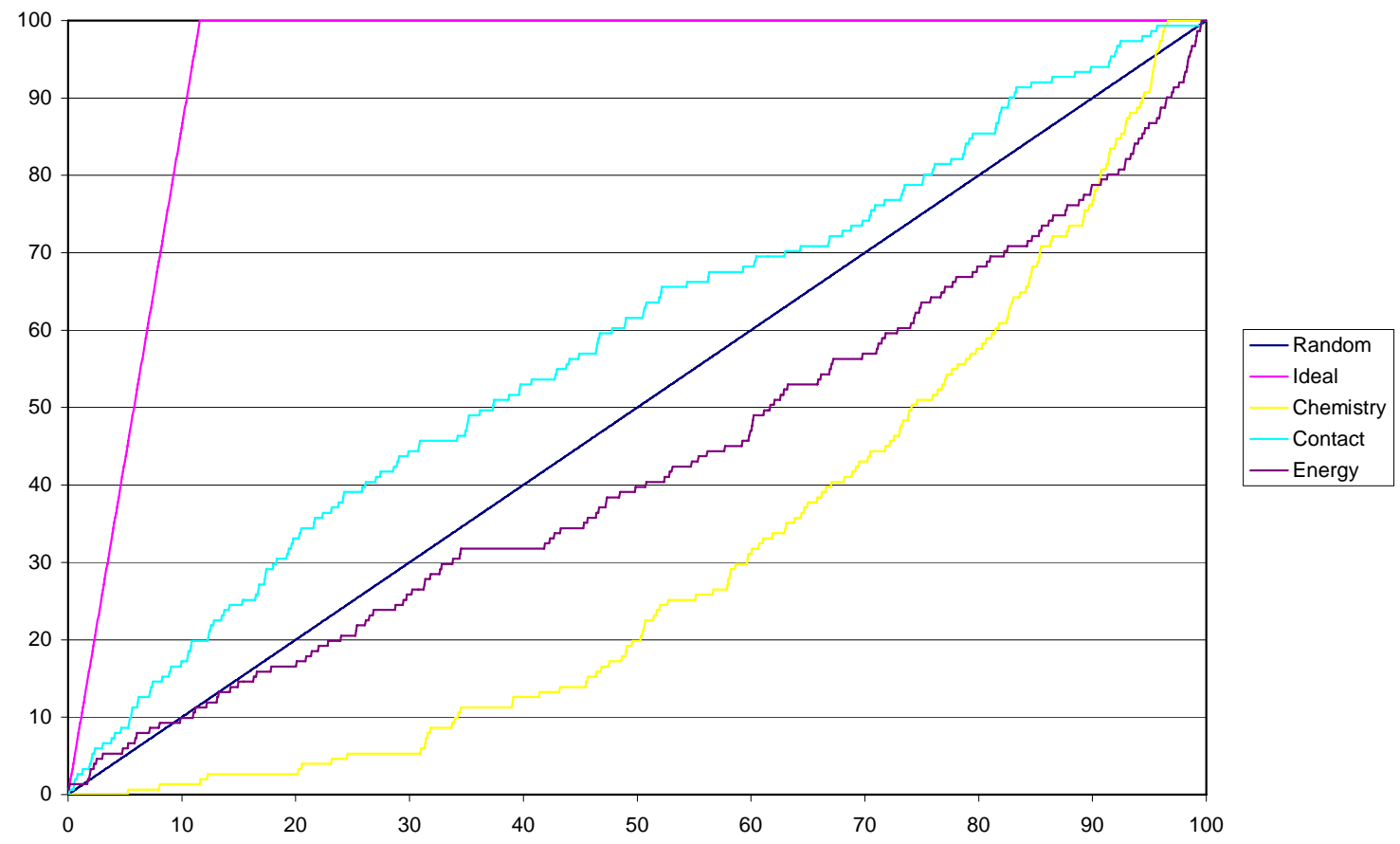

Dock4

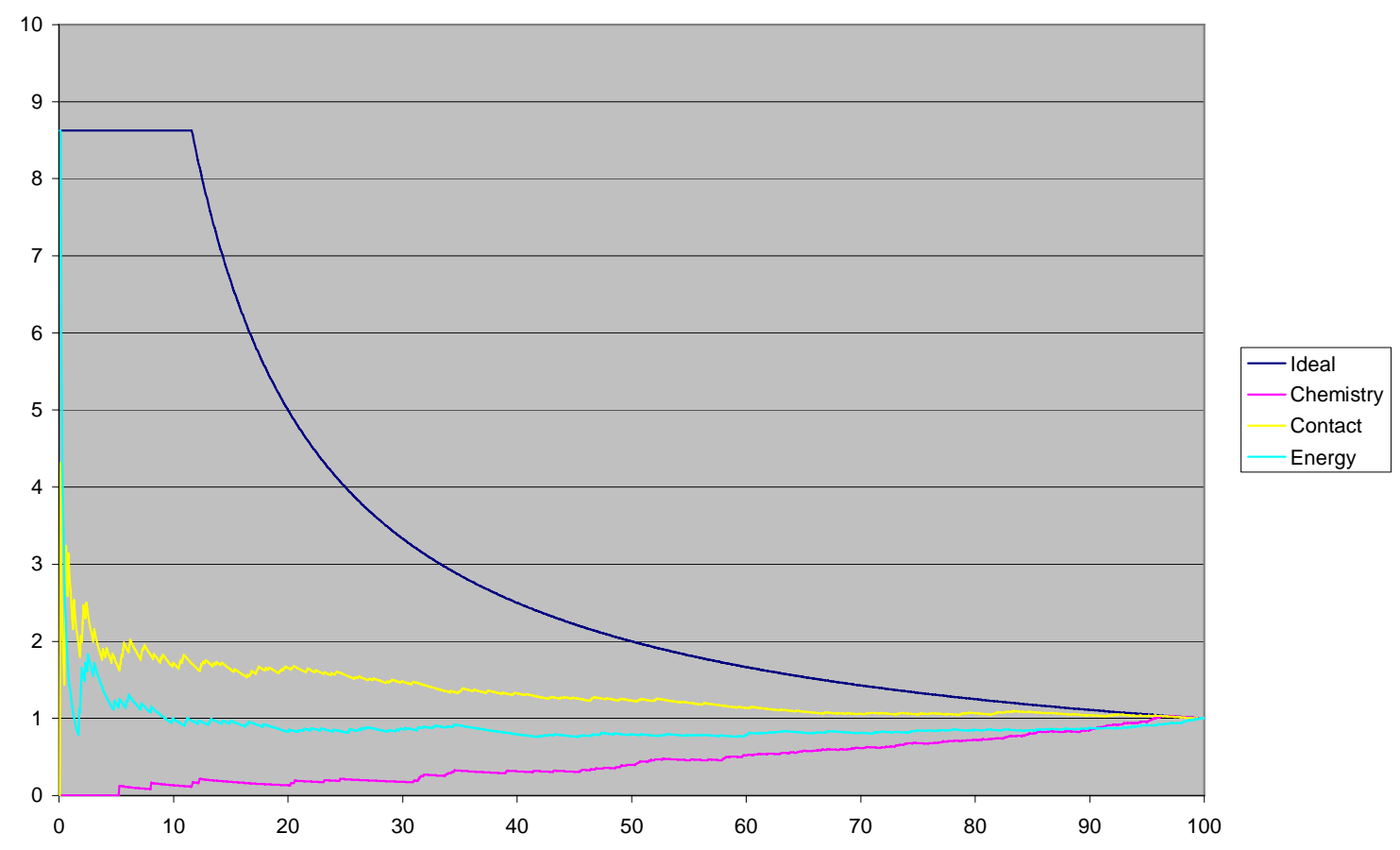




\section{Dockit}

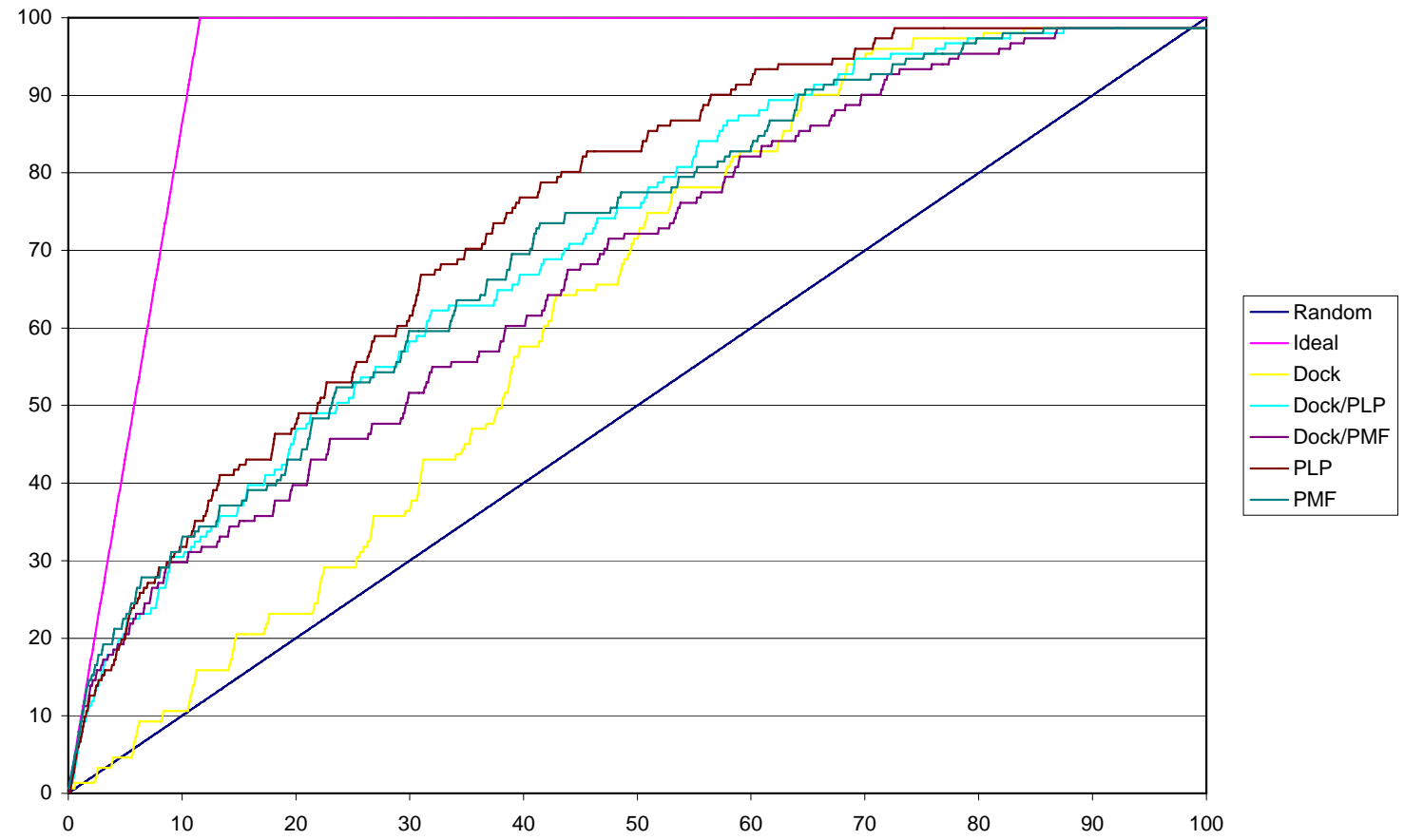

Dockit

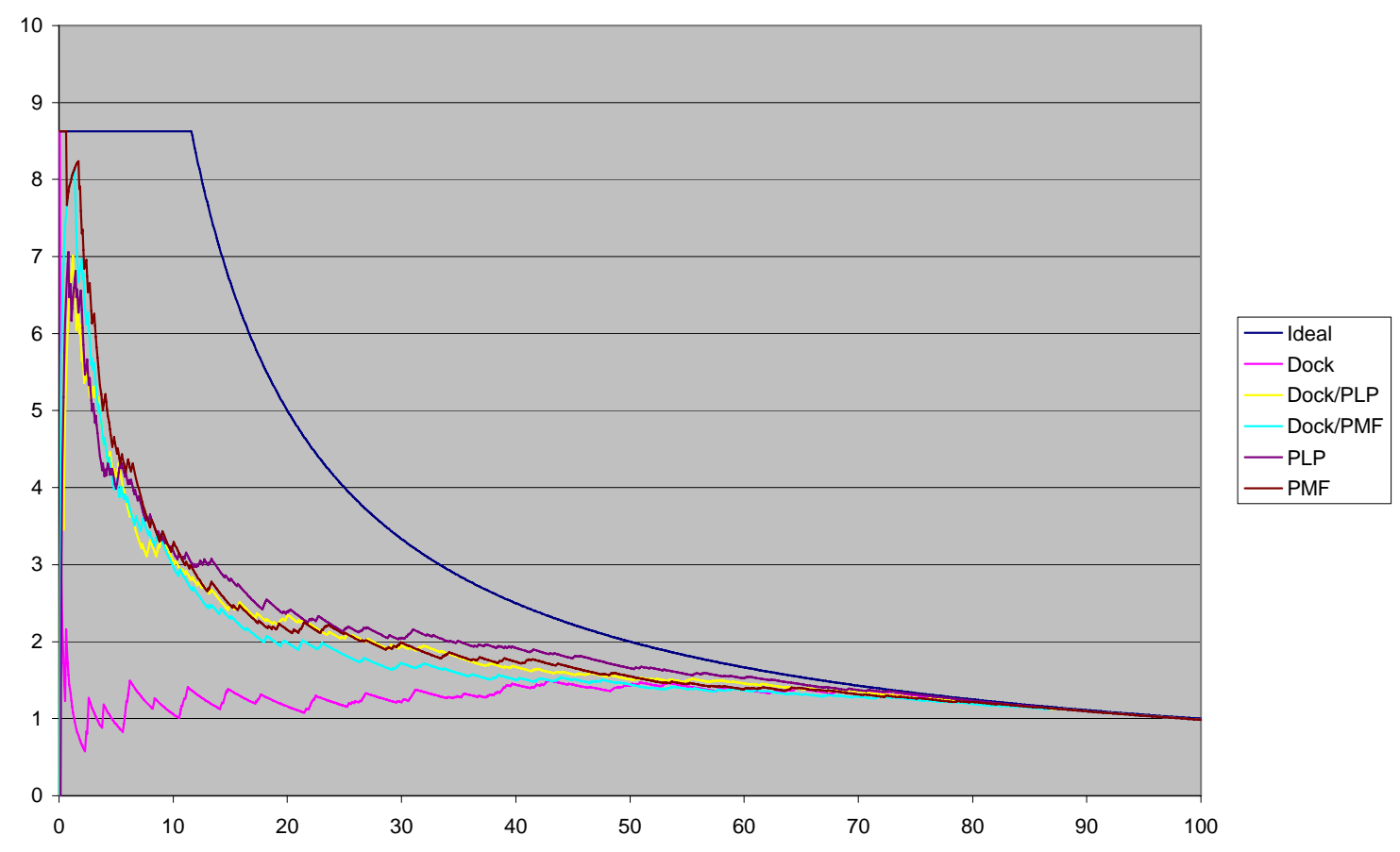


FlexX

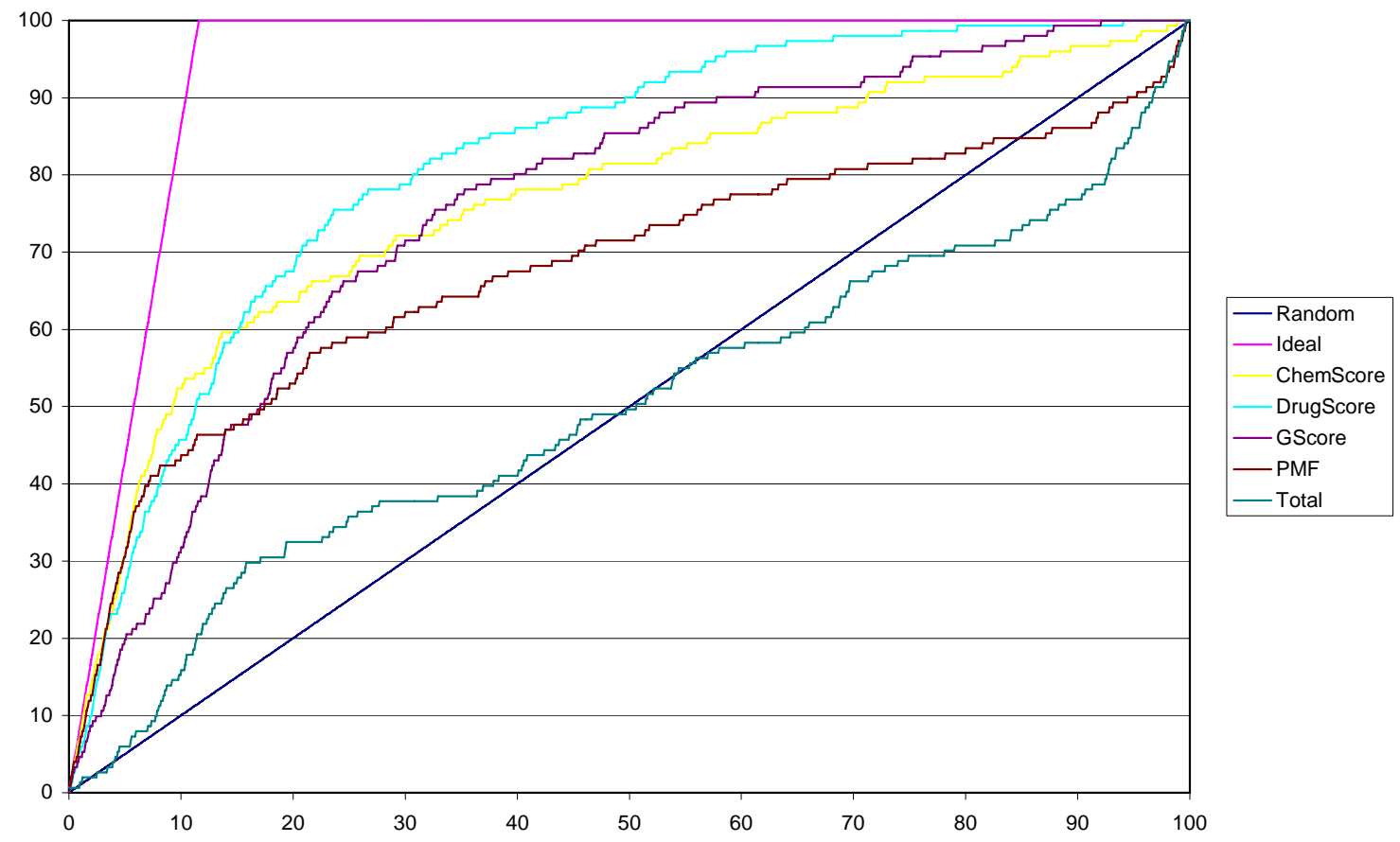

FlexX

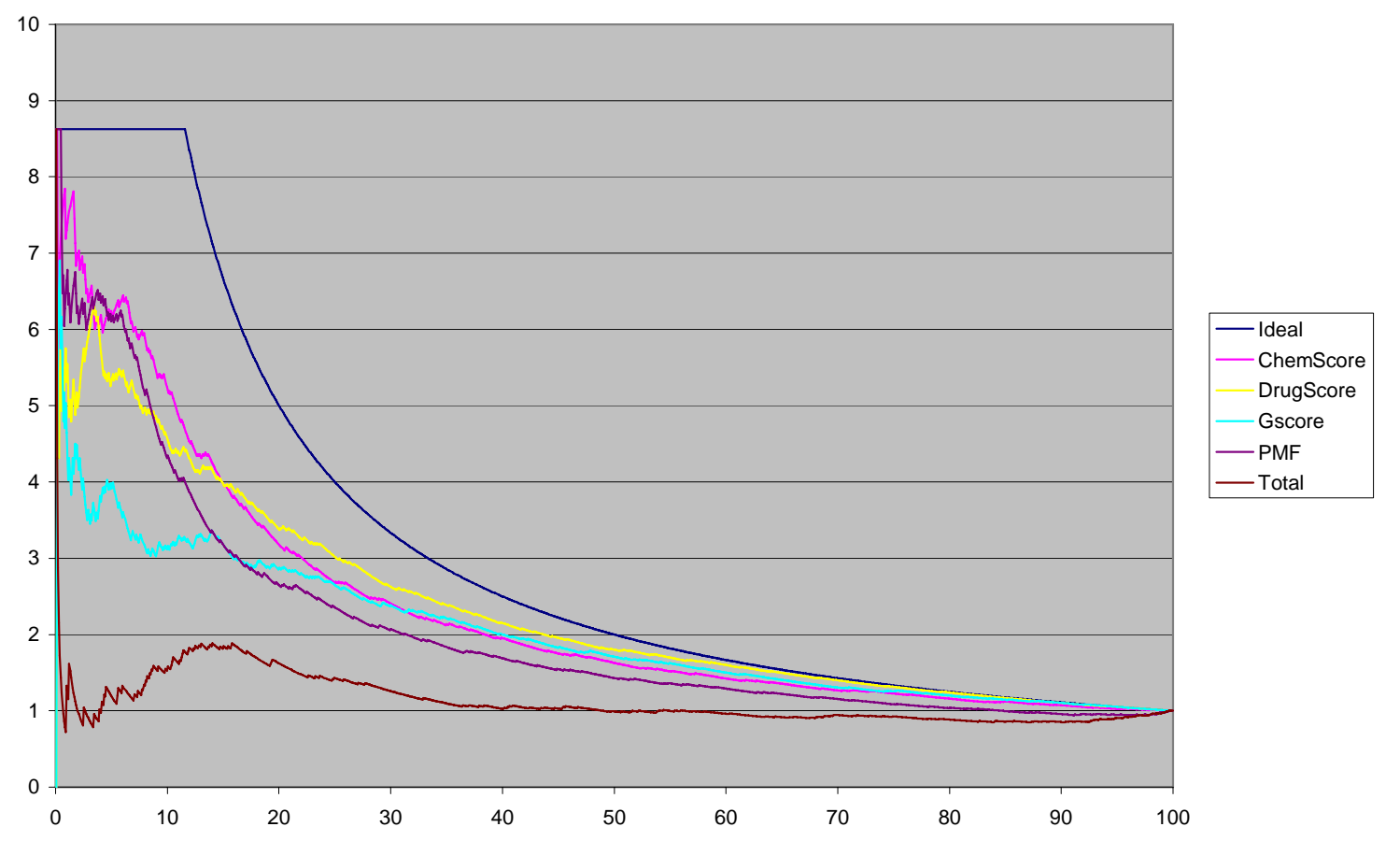




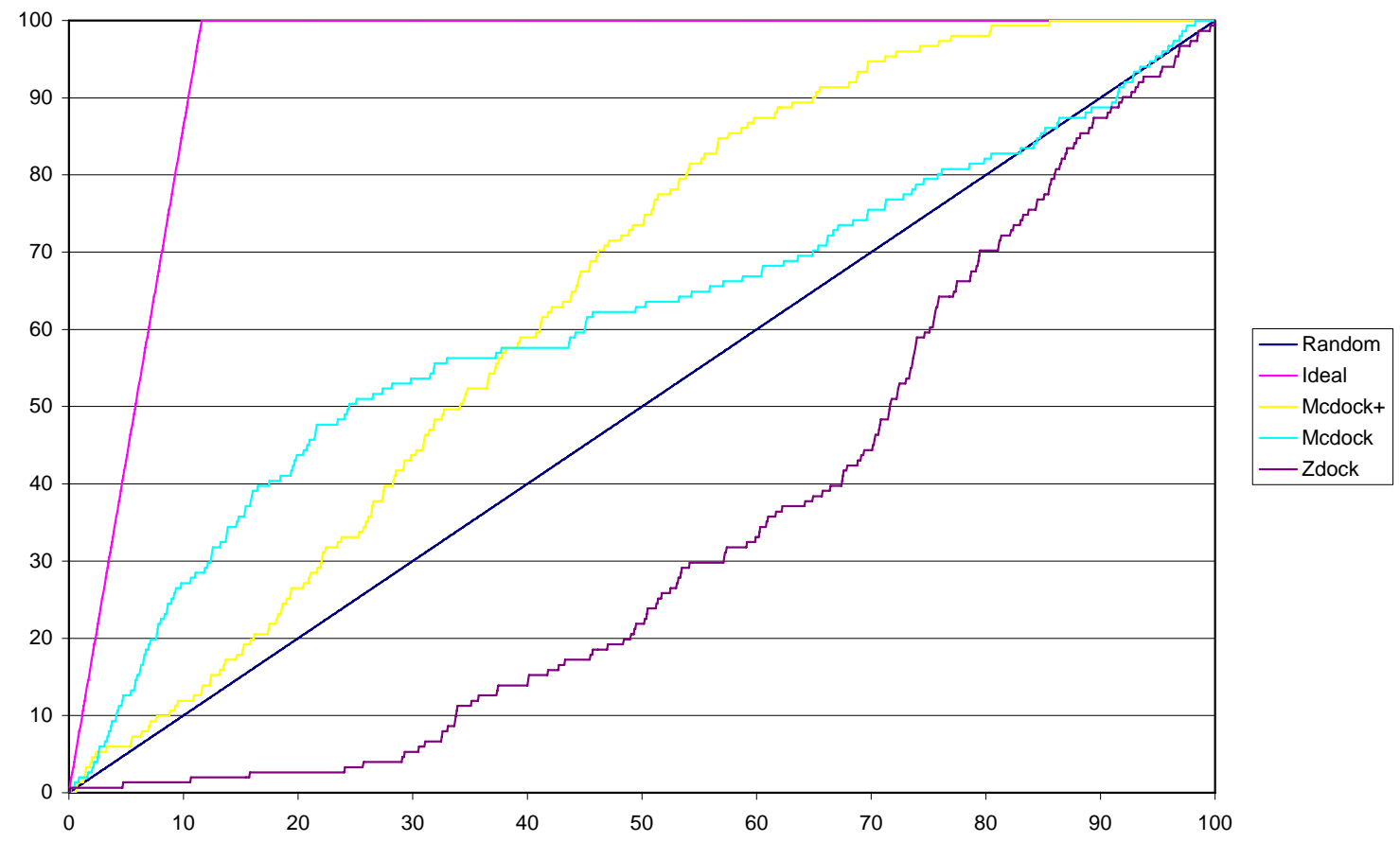

Flo

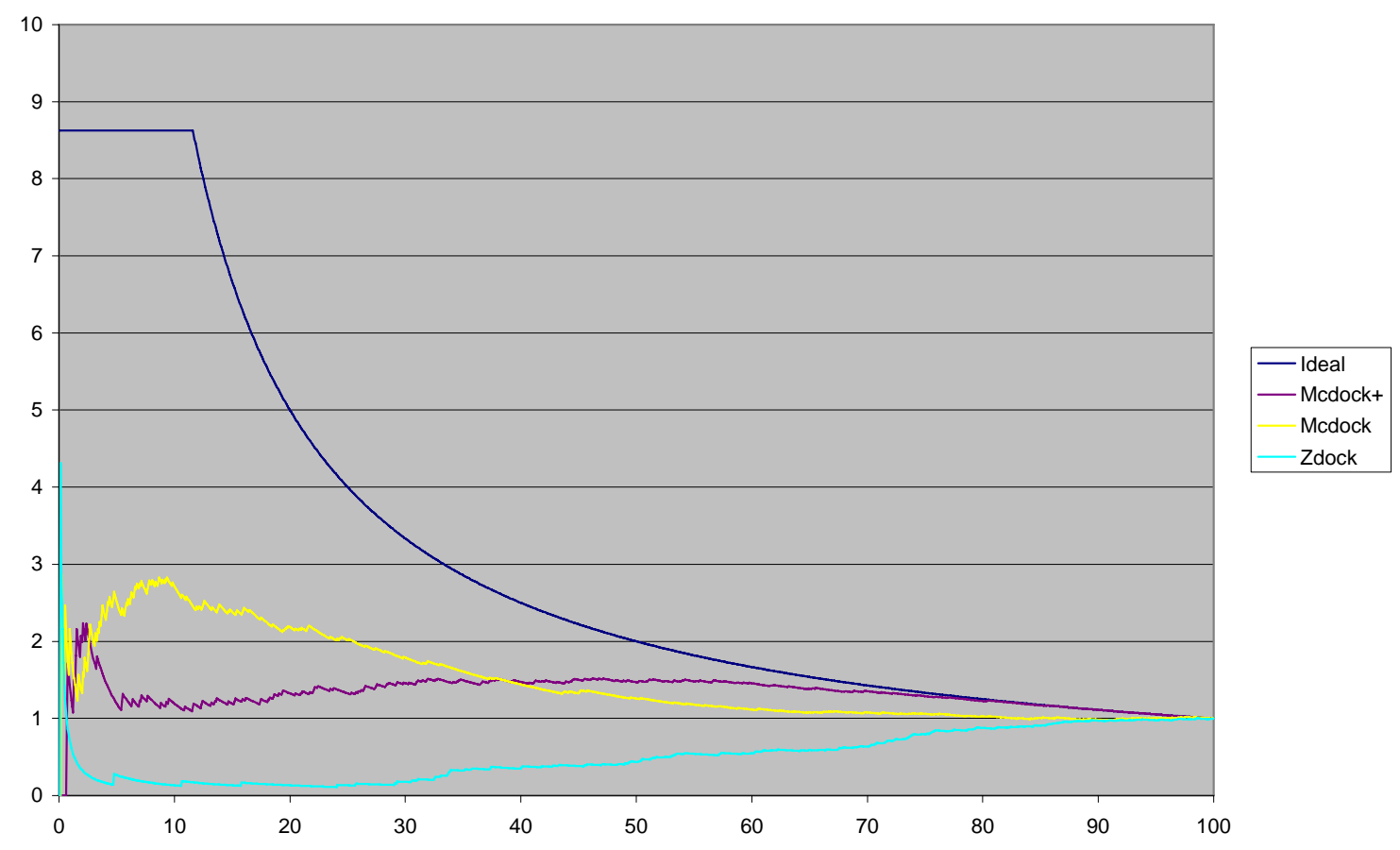


Fred

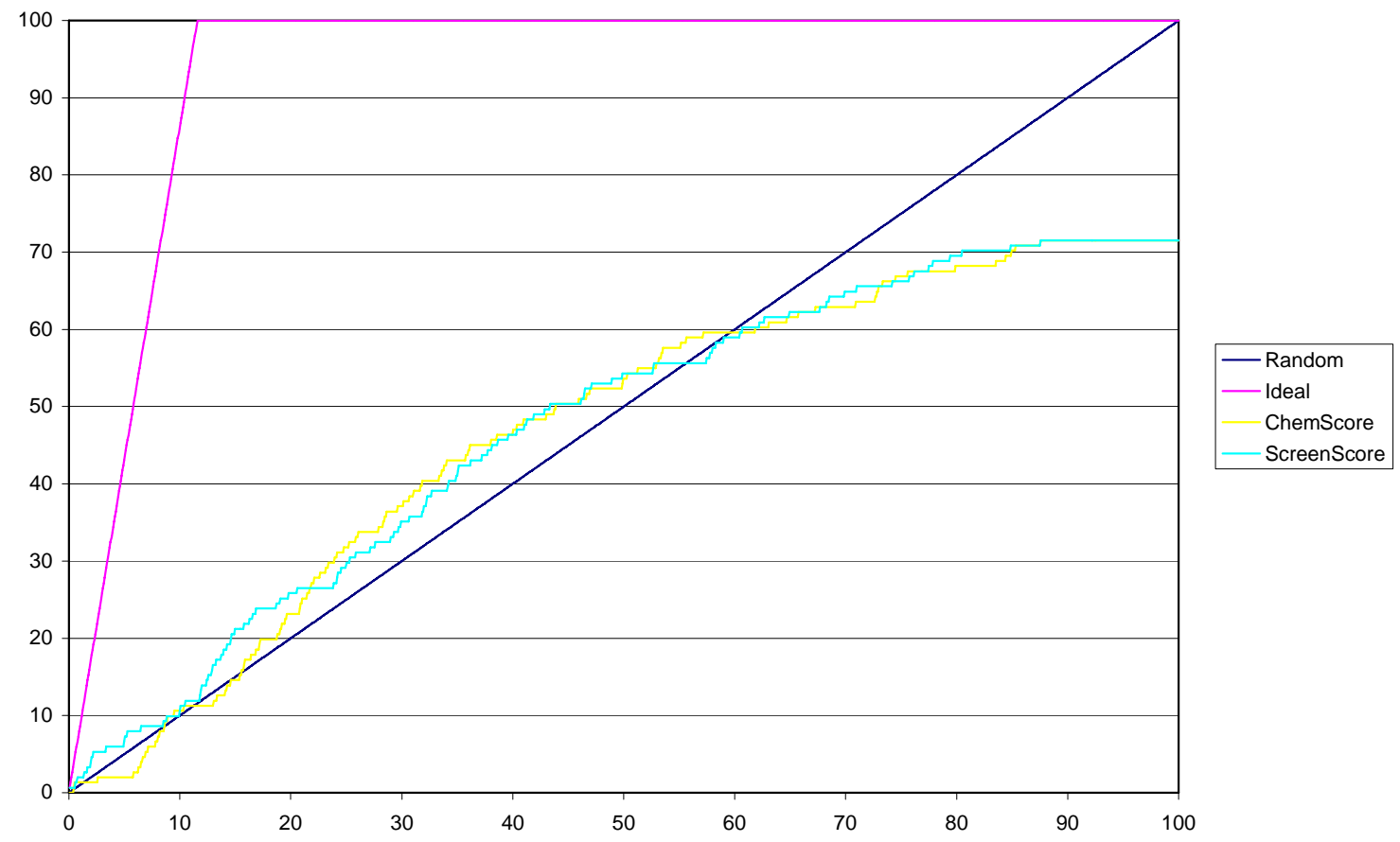

Fred

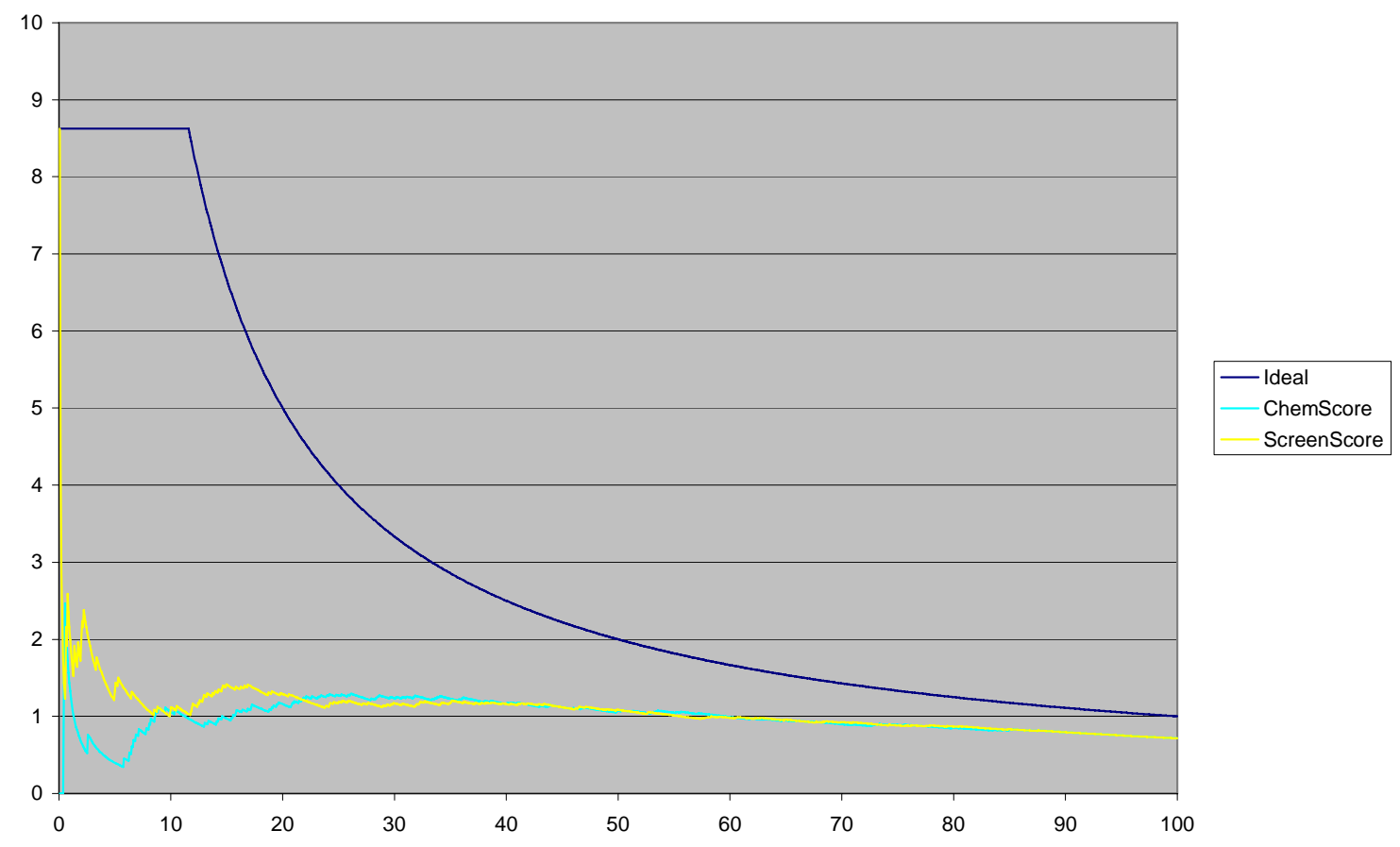




\section{Glide}

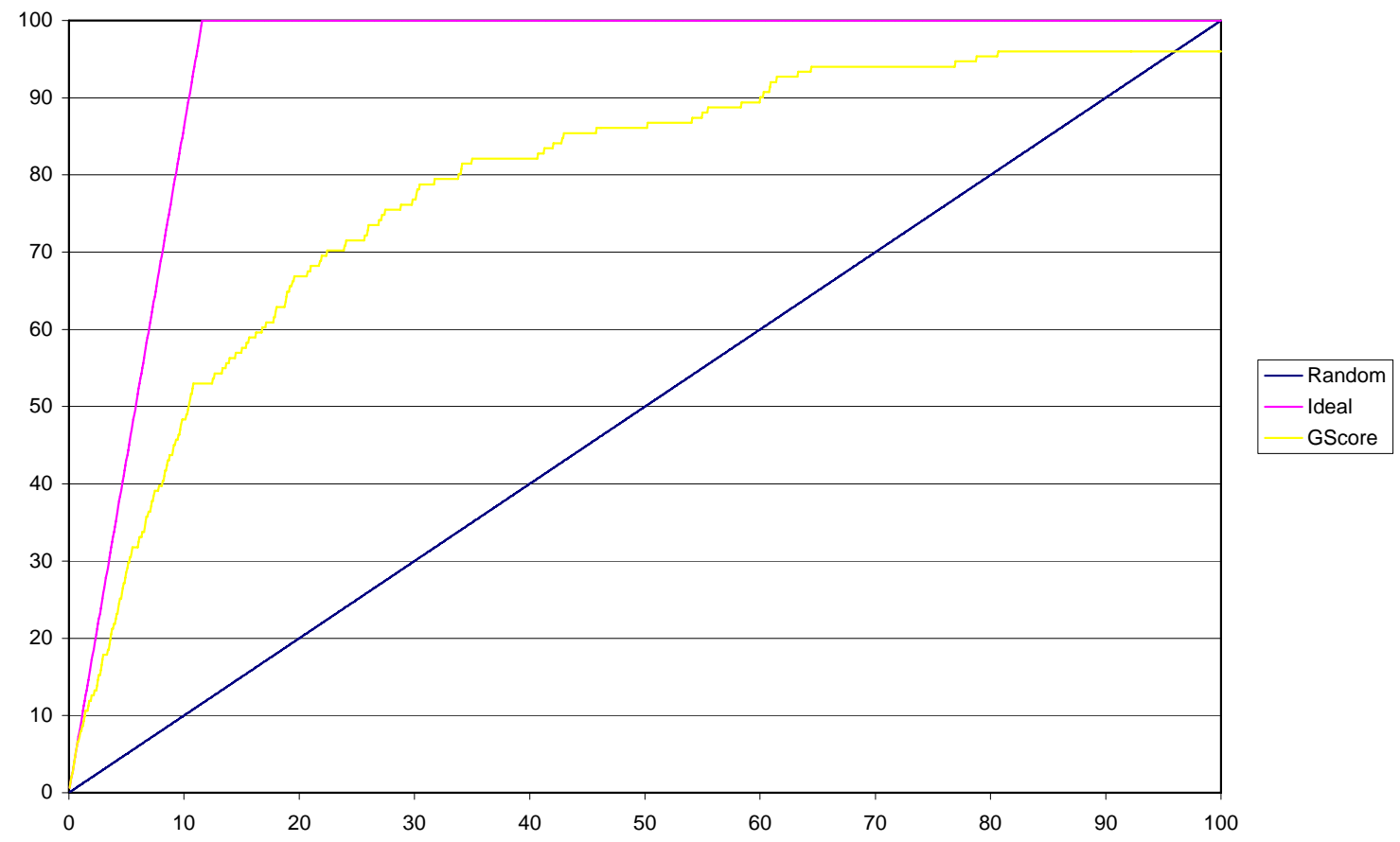

Glide

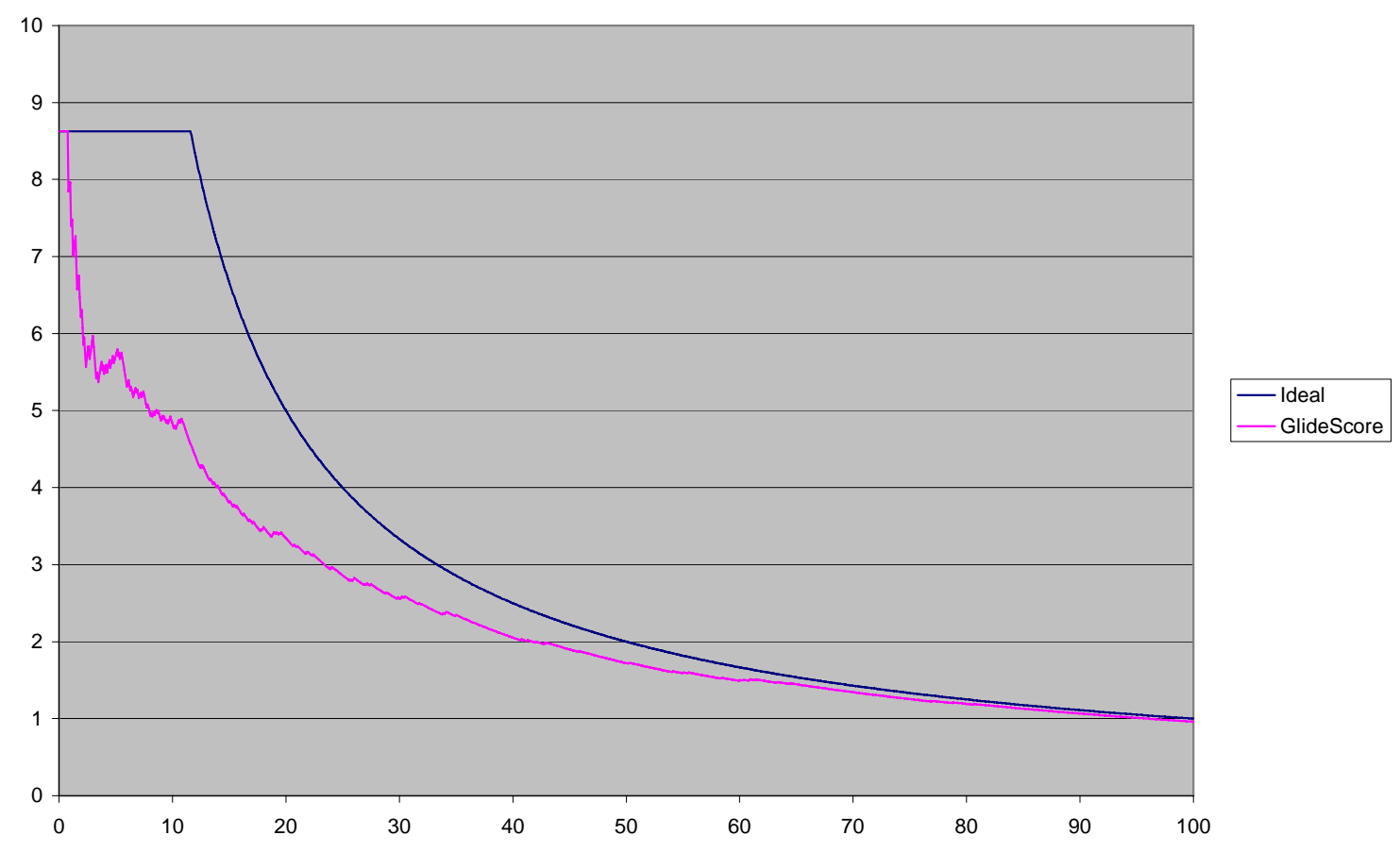


Gold

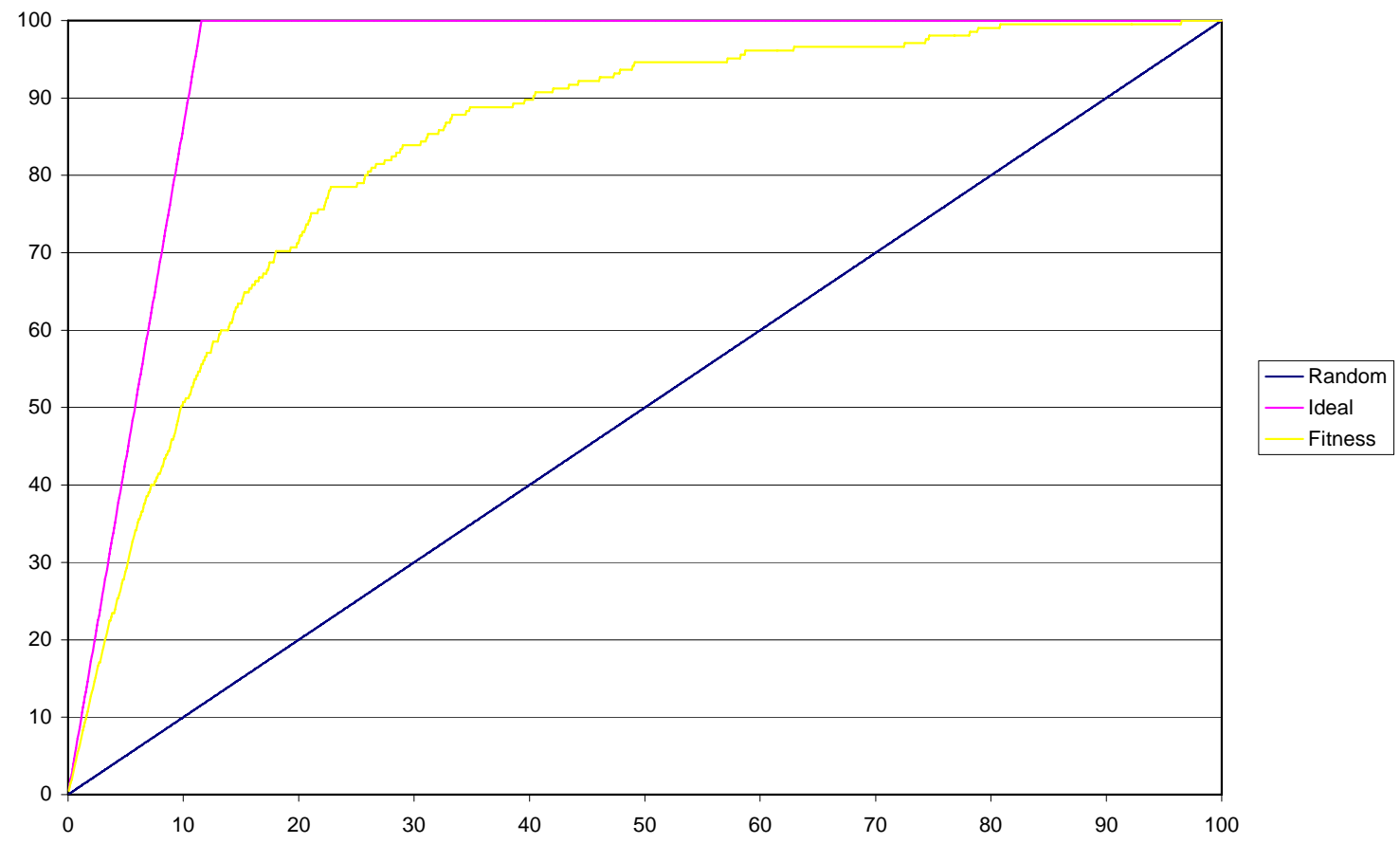

Gold

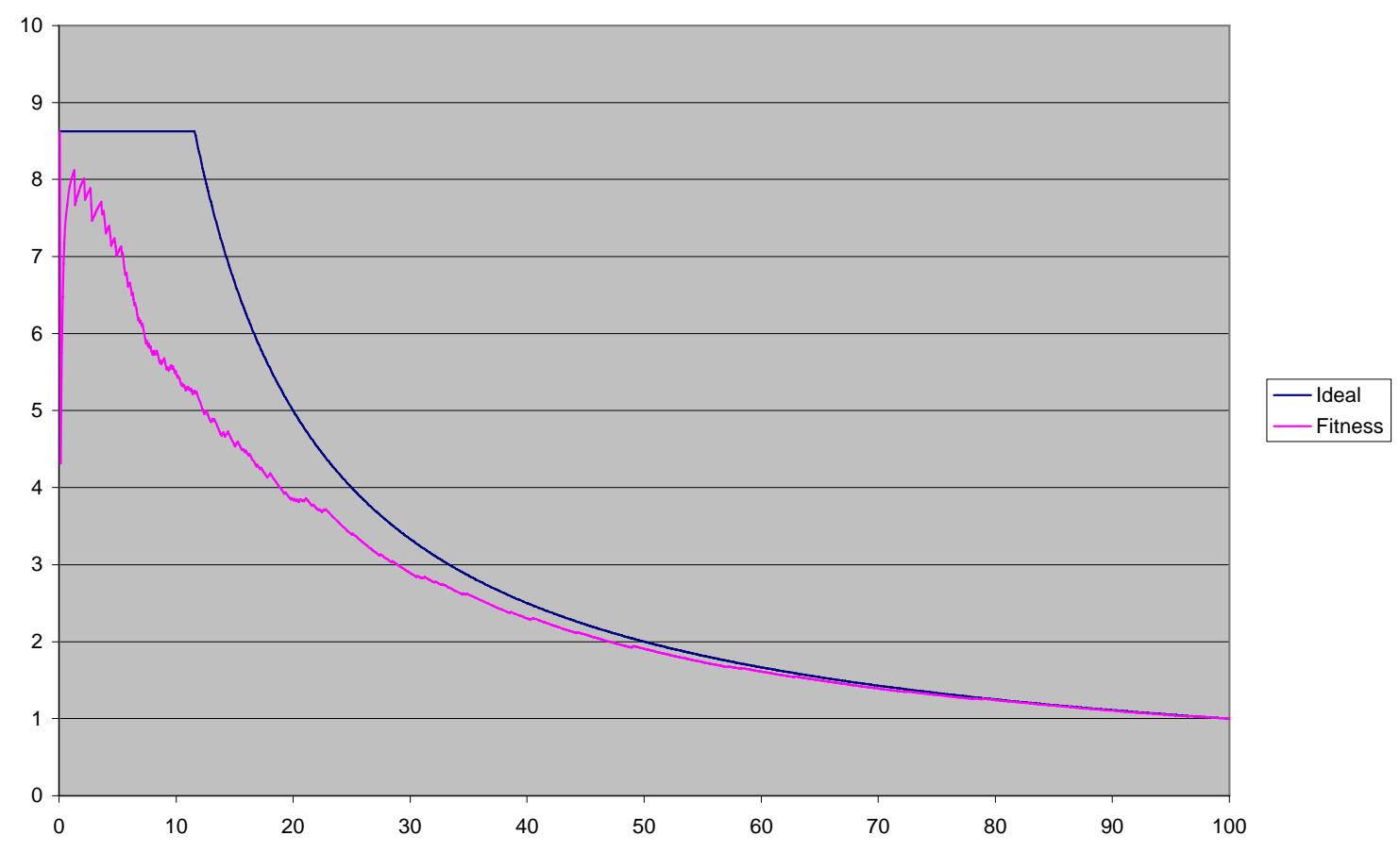


LigFit

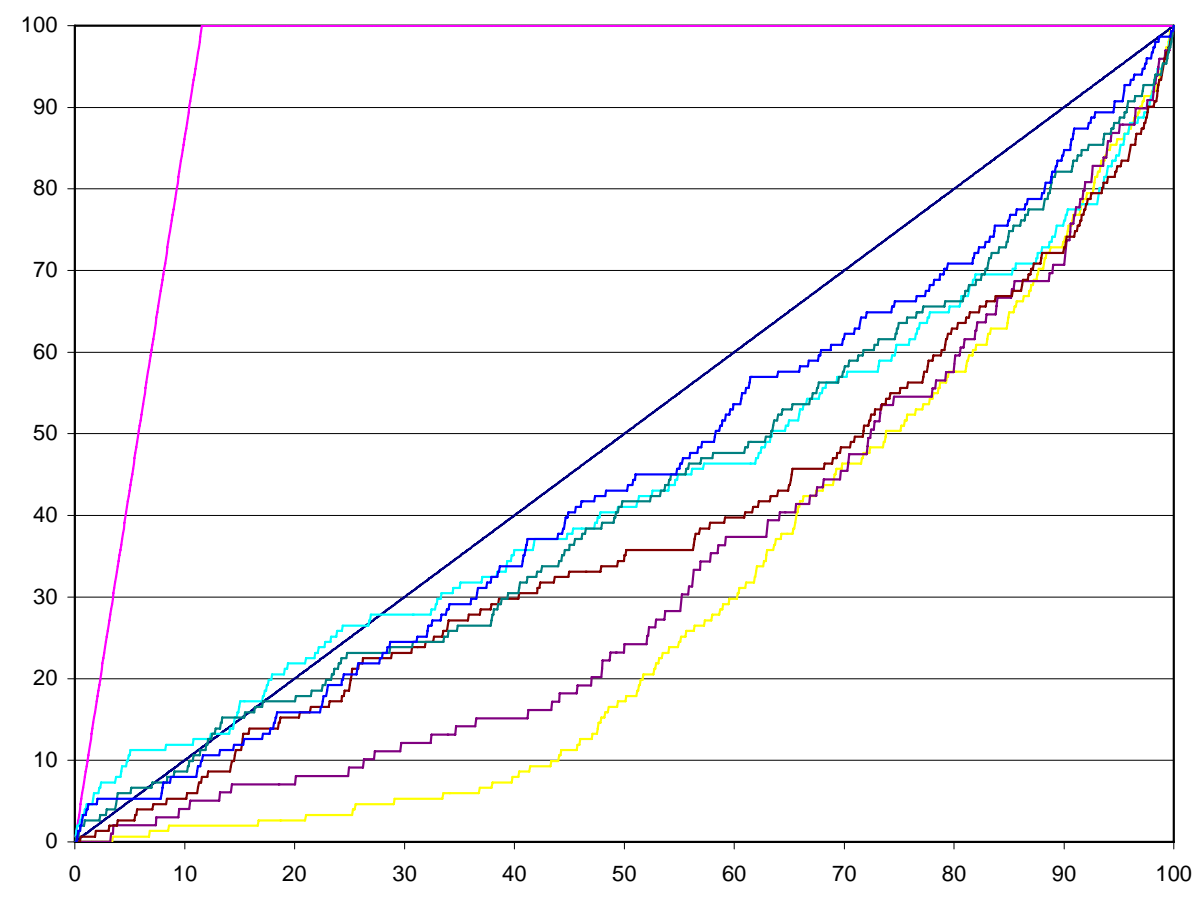

\begin{tabular}{|l|}
\hline - Random \\
- Ideal \\
DrugScore, CFF \\
- DrugScore, Dreiding \\
- Ligscore 2, CFF \\
- Ligscore 2, Dreiding \\
- PMF, CFF \\
- PMF, Dreiding \\
\hline
\end{tabular}

LigFit

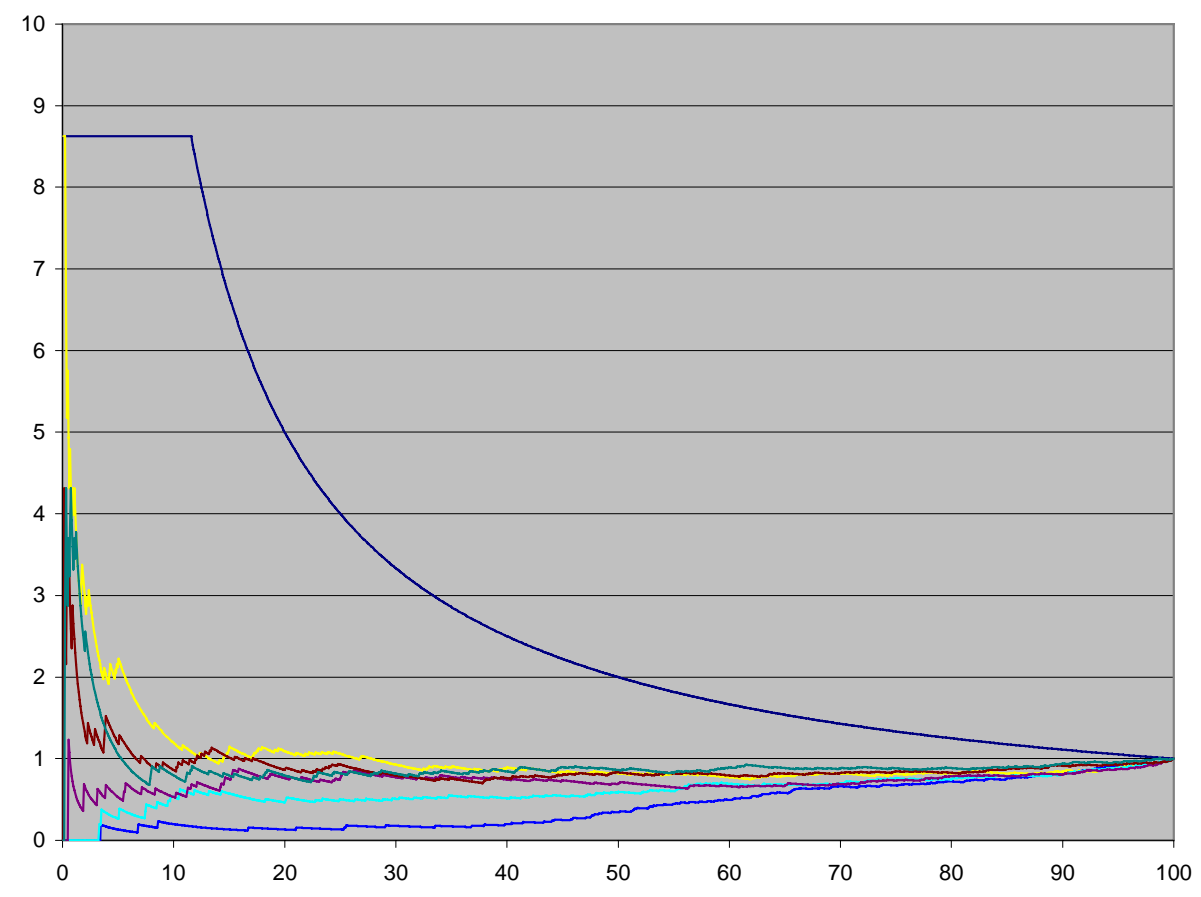


MOE
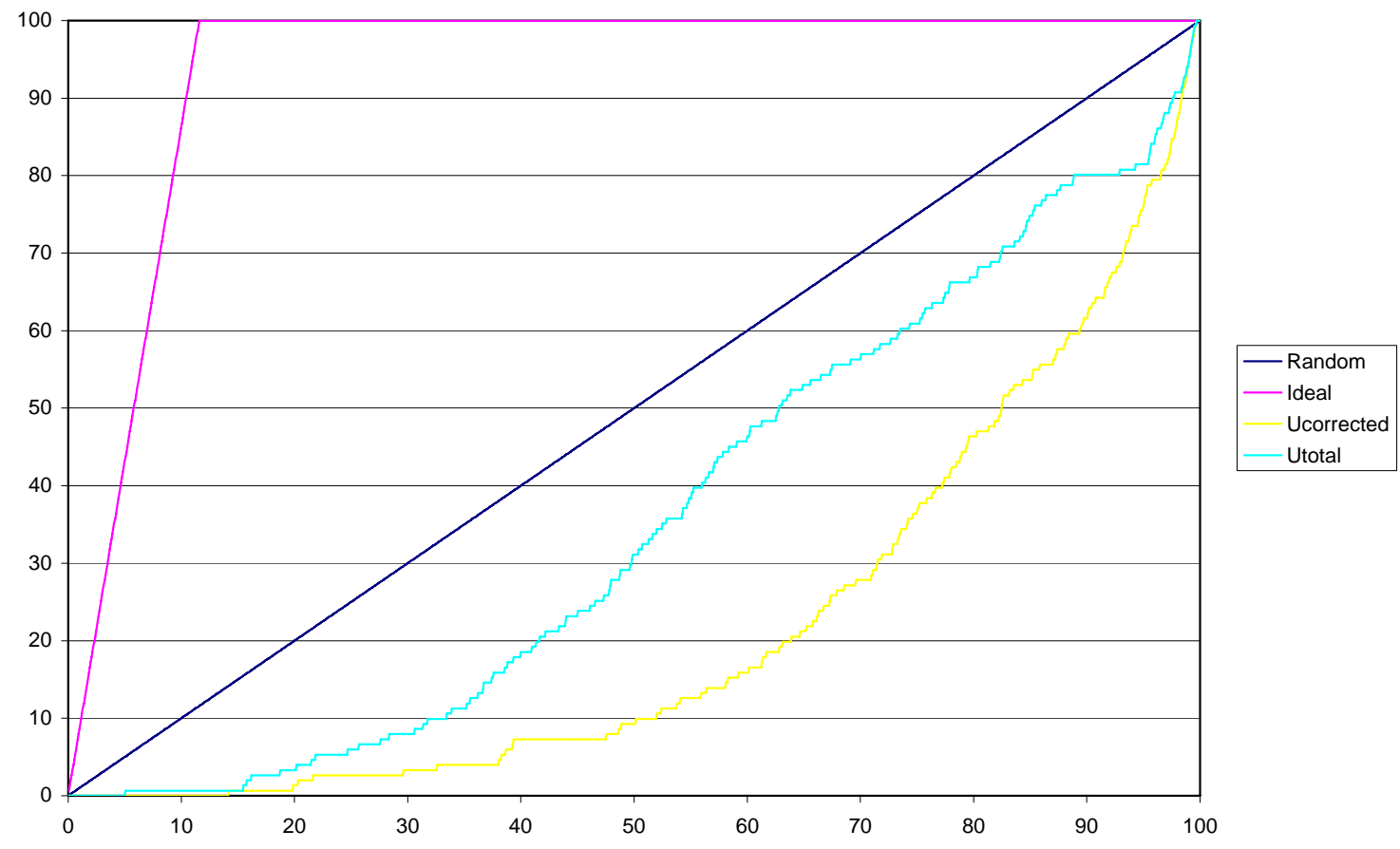

MOE

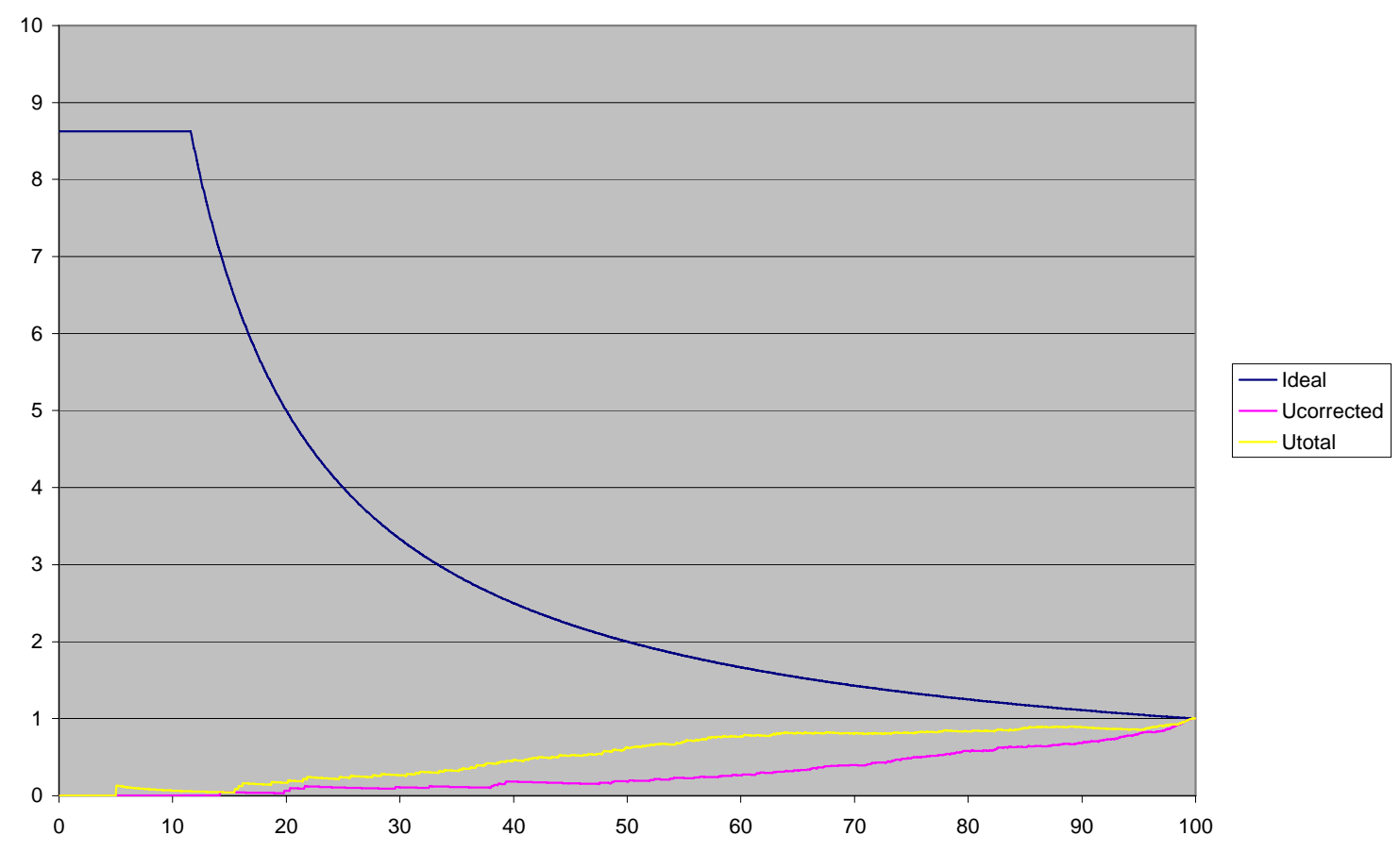


MVP

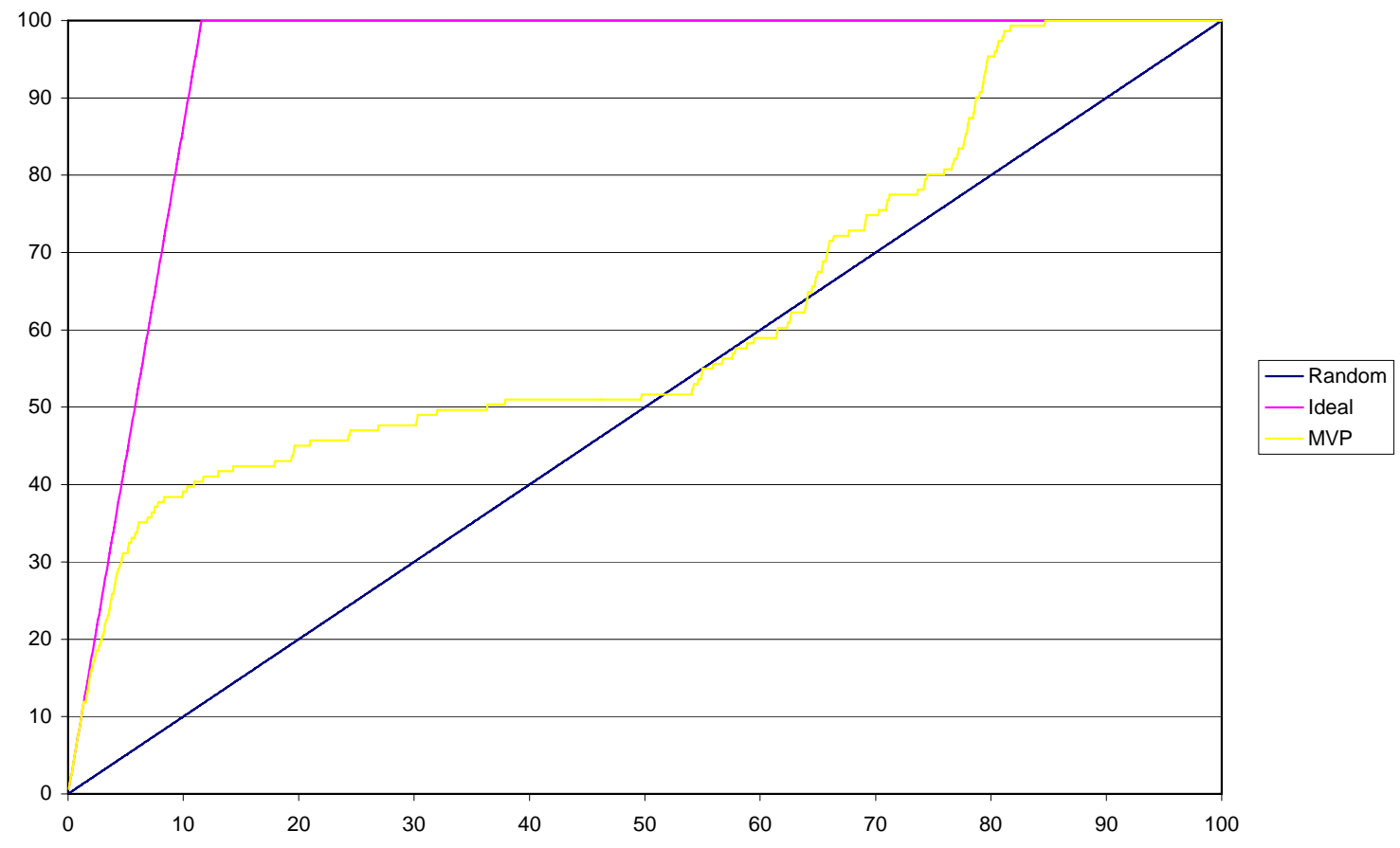

MVP

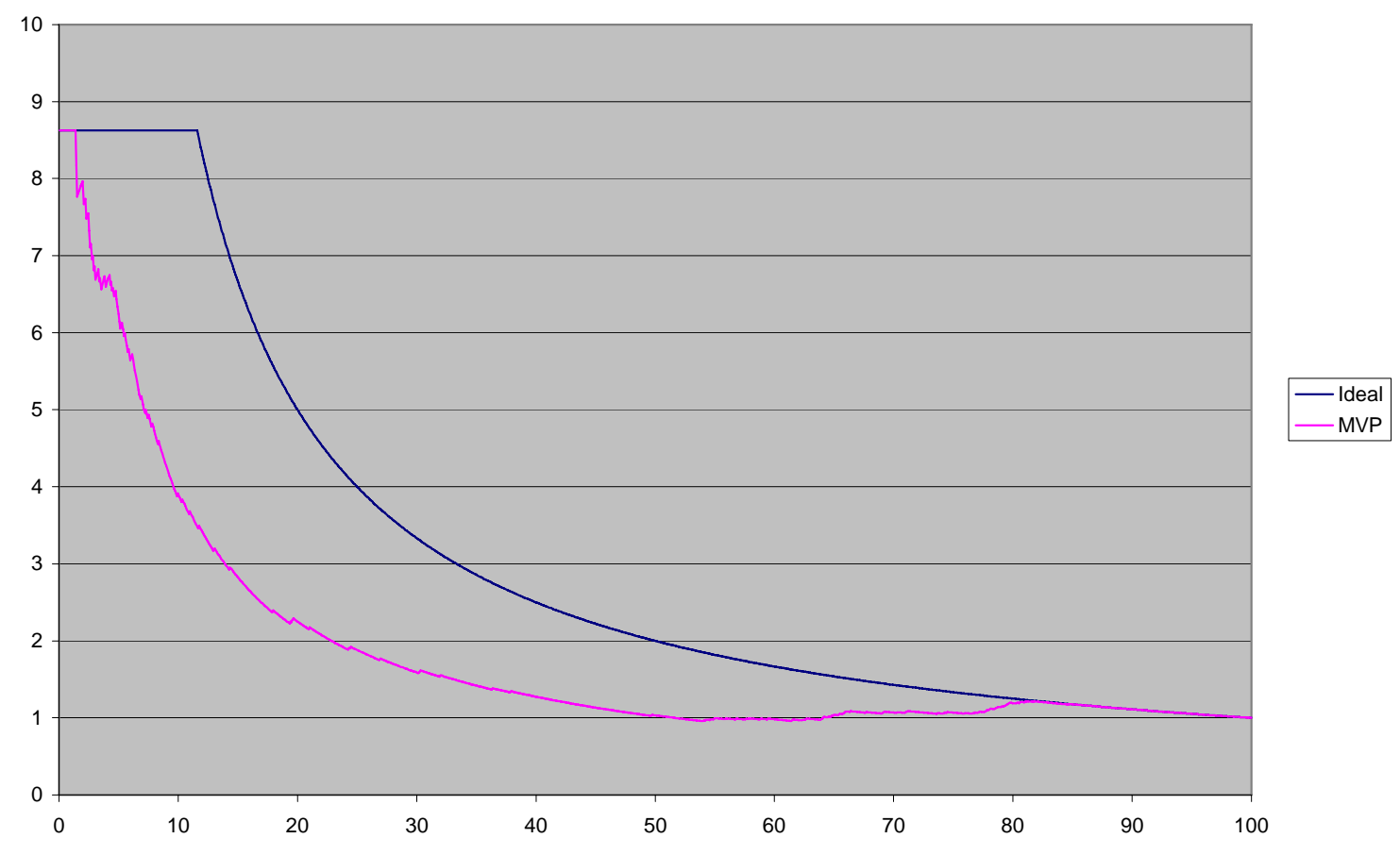

